\title{
IDENTIFICATION OF ORBITAL OBJECTS BY SPECTRAL ANALYSIS AND OBSERVATION OF SPACE ENVIRONMENT EFFECTS
}

\author{
A Thesis \\ presented to \\ The Faculty of California Polytechnic State University \\ San Luis Obispo
}

\author{
In Partial Fulfillment \\ Of the Requirements for the Degree \\ Master of Science in Aerospace Engineering \\ By \\ Jason Bertrand Rapp
}

August 2012 
(C) 2012

Jason B Rapp

ALL RIGHTS RESERVED 
COMMITTEE MEMBERSHIP

TITLE: IDENTIFICATION OF ORBITAL OBJECTS BY SPECTRAL ANALYSIS AND OBSERVATION OF SPACE ENVIRONMENT EFFECTS

AUTHOR: Jason B Rapp

DATE SUBMITTED: August 2012

$\begin{array}{ll}\text { COMMITTEE CHAIR: } & \text { Dr. Kira J. Abercromby } \\ \text { COMMITTEE MEMBER: } & \text { Dr. Eric A. Mehiel } \\ \text { COMMITTEE MEMBER: } & \text { Dr. David S. Mitchell } \\ \text { COMMITTEE MEMBER: } & \text { Dr. Heather M. Cowardin }\end{array}$ 


\begin{abstract}
IDENTIFICATION OF ORBITAL OBJECTS BY SPECTRAL ANALYSIS AND OBSERVATION OF SPACE ENVIRONMENT EFFECTS
\end{abstract}

Jason Bertrand Rapp

This report presents an investigation and development of the methods for orbital object identification. Two goals were accomplished in this master's thesis; the development of a method of inverting material proportions from an object's combined spectrum, and the investigation of methods and initialization of measurement of space environment effects on spectral features of common spacecraft materials. A constrained least squares approach was chosen for inverting spectral proportions from the combined spectra. The final results fall within 1 $15 \%$ of the original spectrum, depending on the quality and noise levels of the original spectrum. Additionally, the effects of outgassing and atomic oxygen erosion were measured using the vacuum chamber facilities at California Polytechnic State University and are to be used as a basis for future identification of orbital debris. To have a fully functional model for accurately identifying space objects, both parts are needed: a set of space environment effect measurements as a basis for the identification model (for use on objects exposed to the space environment), and the identification model to mathematically determine the best fit set of materials. 


\section{ACKNOWLEDGEMENTS}

I would like to thank Dr. Kira Abercromby for her guidance and wealth of information and guidance, without her, this project would have floundered. I would also like to extend my gratitude to Max Glicklin and David Knapp for their tireless work in repairing and operating the vacuum chambers needed for this project, along with Cody Thompson. Additionally, I would like to thank Dr. Eric Mehiel, Dr. Heather Cowardin, and Dr. David Mitchell for their advice, suggestions, and critiques. Most importantly, I would like to thank my wife, Rebecca Rapp, for her unending support, love, and help throughout this project. 


\section{Table of Contents}

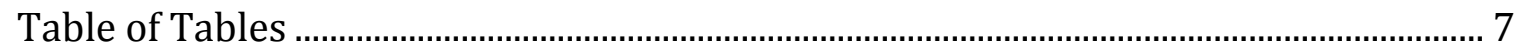

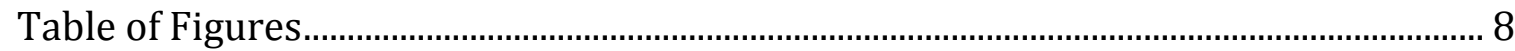

1 - Introduction and Background...................................................................................... 1

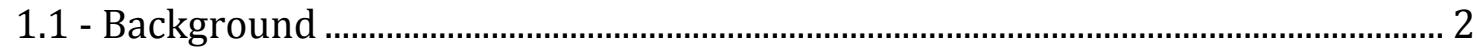

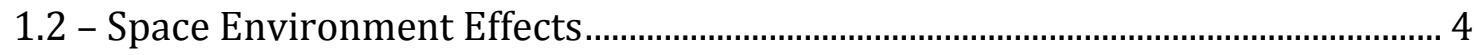

1.3 - Spectroscopy and Spectral Analysis ..................................................................... 7

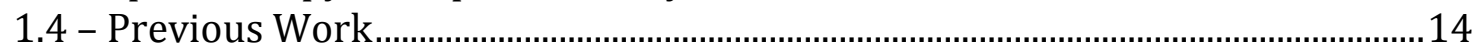

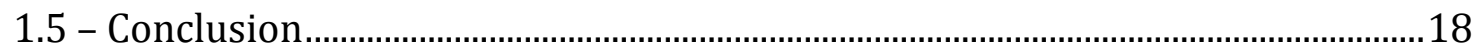

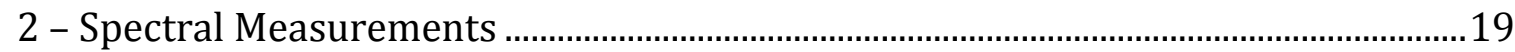

2.1 - Standard Procedure and CubeSat Measurements ..................................................21

2.2 - Outgas Procedure and Measurements ................................................................... 35

2.3 - Atomic Oxygen Procedure and Measurements ……………………………........52

2.4 - Improvements ……………………………………………………………....55

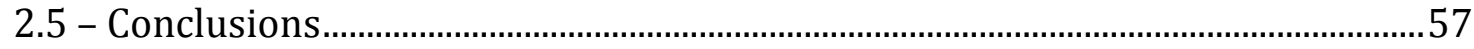

3 - Spectral Unmixing ………………….........................................................................59

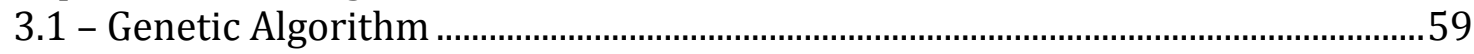

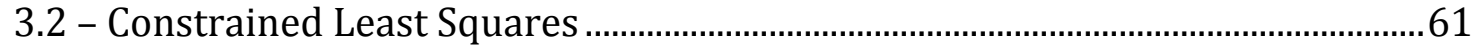

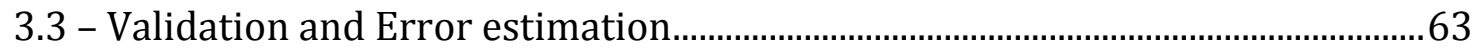

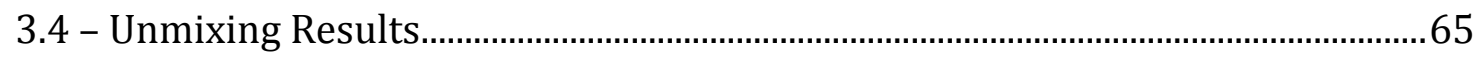

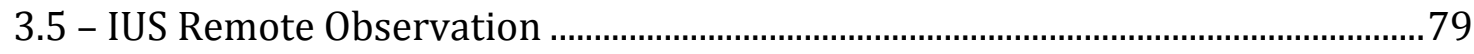

3.6 - Unmixing applied to ID of outgassed materials ................................................85

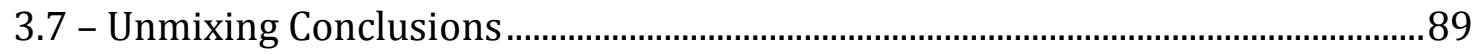

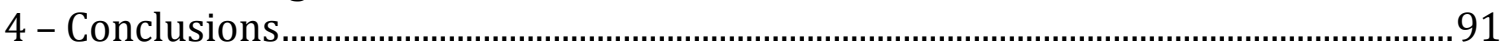

4.1 - Lessons Learned ...............................................................................................91

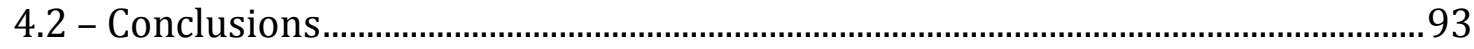

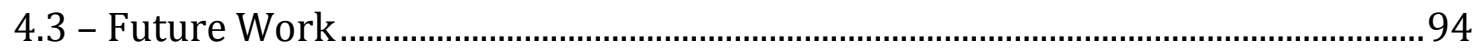

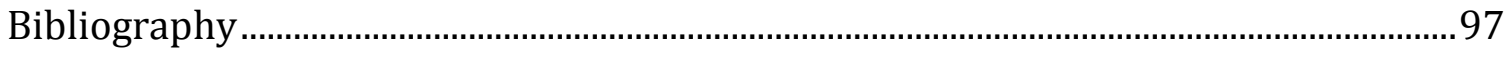

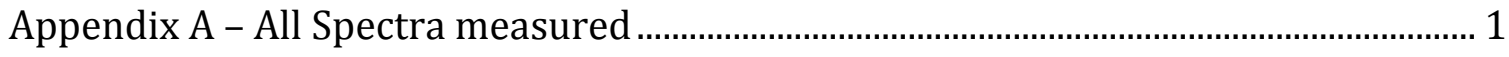

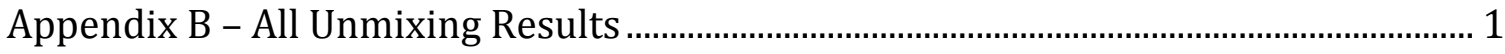

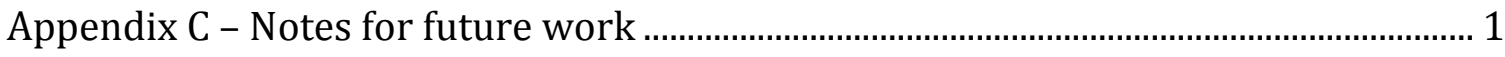

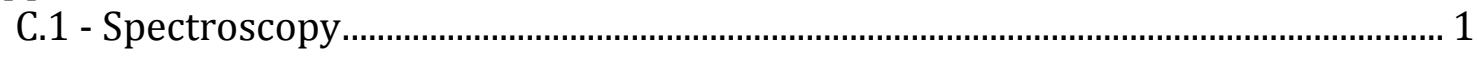

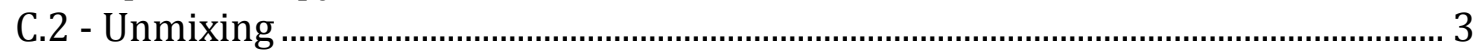




\section{Table of Tables}

Table 1. List of materials for spectral measurements.....................................................24

Table 2. Materials on which outgassing effects were measured.......................................40

Table 3 Simulated mixing unmixing results with a full vector. ........................................67

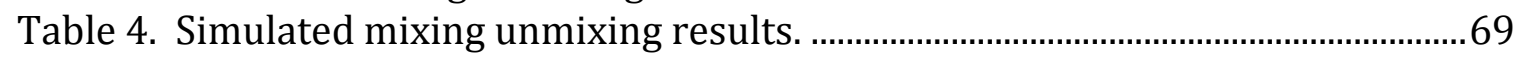

Table 5 Unmixing results for Positive X side of AubieSat..................................................71

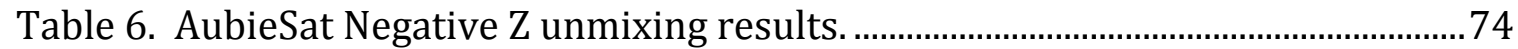

Table 7. HRBE Positive Y side unmixing results.................................................................... 76

Table 8. HRBE Negative Y unmixing results. ....................................................................... 79

Table 9. IUS Rocket Body Measurement (Measurement 1) .............................................82

Table 10. IUS Rocket Body Measurement (Measurement 2) .........................................84

Table 11. 2mil Kapton x Aluminum (Kapton Side) Unmixing Identification...............86

Table 12. Aluminum Beta Cloth (Aluminum Side) Unmixing Identification...............88 


\section{Table of Figures}

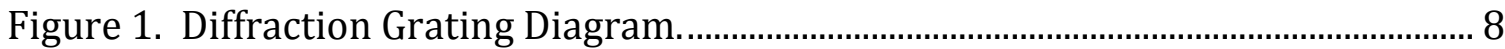

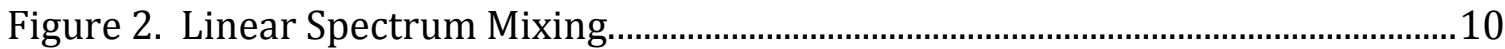

Figure 3. Bidirectional Reflectance Distribution Function (BRDF). ...............................12

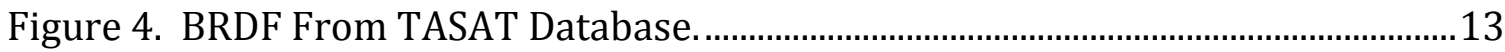

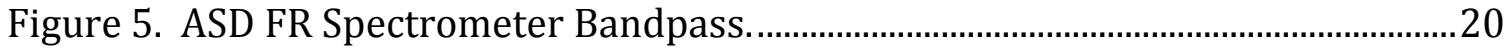

Figure 6. Typical Configuration for ASD FieldSpec Spectrometer. .................................21

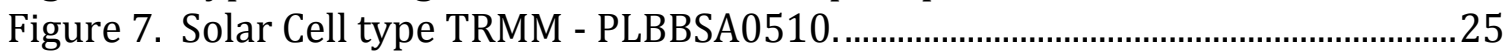

Figure 8. Spectra of the second category of solar cell, members from AubieSat

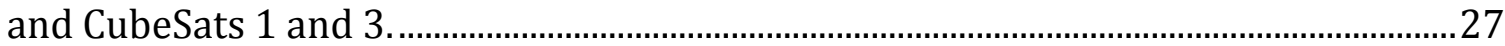

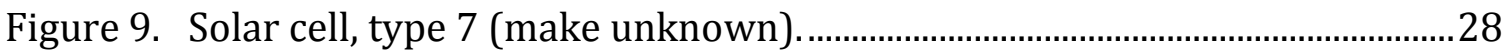

Figure 10. Comparison of solar cell TRMM-PLBBSA0510 to measurement HRBE

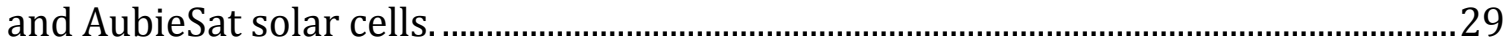

Figure 11. Directional measurement of Solar Cell TRMM spectra..................................30

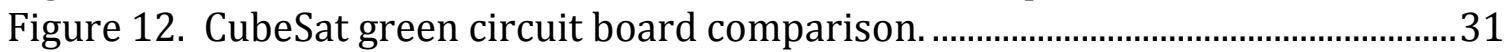

Figure 13. AubieSat coated / uncoated green circuit board comparison.......................32

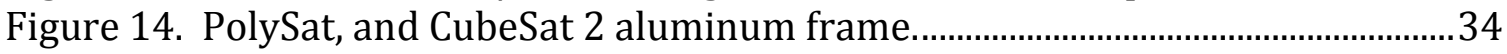

Figure 15. Comparison of anodized aluminum frames. ................................................... 35

Figure 17. Wet Erase mat used as location marking tool. ...................................................38

Figure 18. Kapton outgas test results from initial testing in the glass bell jar

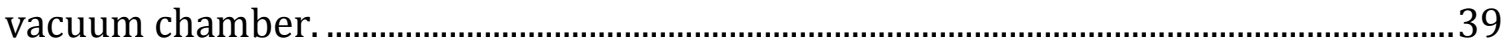

Figure 19. Aluminzed Beta Cloth reference photos. ........................................................... 41

Figure 20. Aluminum Beta Cloth outgas measurements, Beta Cloth side. ....................42

Figure 21. Aluminum Beta Cloth outgas measurements, Aluminum side.....................42

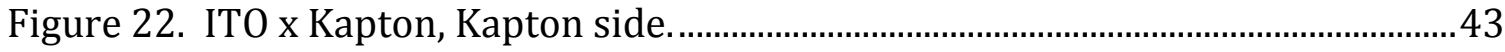

Figure 23. Reference photo for Germanium x Black Kapton............................................. 45

Figure 24. Germanium x Black Kapton Film outgas, Germanium side..........................45

Figure 25. Teflon $x$ Silver Inconel reference photos........................................................ 47

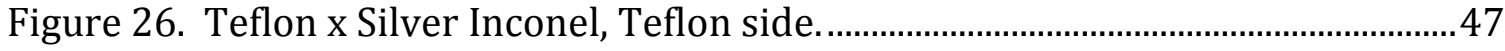

Figure 27. Reference Photos for Aluminum x 2mil Kapton ............................................49

Figure 28. Aluminum x Kapton, Kapton side......................................................................49

Figure 29. PolySat Black Circuit Board outgas test...........................................................51

Figure 30. Atomic Oxygen Erosion effects on Kapton HN control experiment...........53

Figure 31. Absolute Reflectance comparison for Atomic Oxygen Erosion of

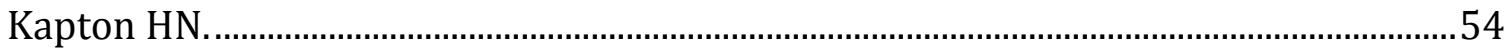

Figure 32. Full vector unmixing results.........................................................................66

Figure 33. Simulated mixing unmixing results with a partial vector...............................68

Figure 34. AubieSat Positive X side reference photo for unmixing. ................................ 70

Figure 35. Unmixing results for Positive X side of AubieSat. ................................................71

Figure 36. Negative y side of the AubieSat cubesat. ............................................................ 73

Figure 37. AubieSat Negative Y unmixing results.................................................................. 73 
Figure 38. HRBE Positive Y reference photograph. ........................................................ 75

Figure 39. HRBE Positive Y side unmixing results........................................................... 76

Figure 40. HRBE Negative Y side reference photograph. .................................................78

Figure 41. HRBE Negative Y unmixing results.................................................................78

Figure 42. Picture of an IUS with both stages in the space shuttle bay (courtesy of NASA) [13].

Figure 43. IUS Rocket Body spectra measurement (Measurement 1)

unmixing results comparison

Figure 44. Possible orientation of the IUS rocket body in Measurement 1 ...................83

Figure 45. IUS Rocket Body spectra (Measurement 2),

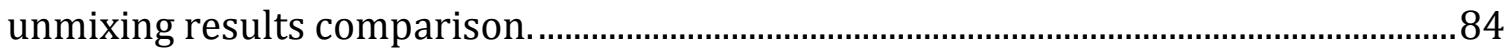

Figure 46. 2mil Kapton x Aluminum (Kapton Side) Unmixing Identification.............86

Figure 47. Aluminum Beta Cloth (Aluminum Side) Unmixing Identification.............88 
Chapter 1 - Introduction and Background | 1

\section{1 - Introduction and Background}

A major consideration in the development of space exploration and earth observation is the identification of objects in orbit around the Earth. Currently, most identification of objects in orbit is done using methods that reveal little about the objects other than size and location. The primary method uses the location and known orbital parameters to verify an object's position by observing it in a predicted position at a predicted time [1]. This works well for known objects, but lacks certainty. To truly identify an object's surface properties, more informative methods are needed. To this end, unmixing spectral features has been investigated.

Spectroscopy is a method used in a variety of fields for the identification of materials. For identification of spacecraft materials it is useful to use reflectance spectroscopy where light reflected from the object is passed into a grating, split angularly by wavelength, and magnitude is measured relative to that wavelength. This produces a material's spectrum, the features of which can be used to identify the material from which the light reflected. The purpose of the work in this thesis is to further explore and develop the methods of identifying objects by the reflected spectra. This is accomplished by developing a method of 'unmixing' or inverting the material proportions from the combined material spectrum. Such a method requires a basis of known materials and accompanying spectra for each material to compare to for identifying the features present in the combined material spectrum. Another important consideration is the effects of the space environment on 
Chapter 1 - Introduction and Background | 2 materials exposed over long duration flights. Depending on the effects present, this can have anywhere from a minimal or negligible effect to significantly changing the reflectance spectrum. Having a basis of not only the material spectra, but the space environment effects on those spectra is an important goal for a comprehensive unmixing program.

The purpose of this report is to explain the methods and process used to unmix material proportions, and to report on the data acquired and methods developed for measuring the space environment effects on material spectra. The report will begin by going into the background and theory of spectroscopy and spectral analysis, then continues with the methodology and conclusions of the space weathering spectral measurements. From there, the methods, validation, and results of the spectral unmixing work will be covered, followed by conclusions and future work.

\section{1 - Background}

Debris is a growing problem, as the number of launches and spacecraft in orbit grows the number of collisions and unintentional debris grows as well. All types of orbital objects, including spacecraft and debris, can be identified through spectroscopy, though it is most useful to apply unmixing to the class of object that contains several distinct materials on its surface. The unmixing process will identify 
Chapter 1 - Introduction and Background | 3 single material spectrum, so it has application to all orbital objects, including debris. A spectral unmixing program will allow the rapid identification of orbital objects, assuming the material's spectrum is in the database. The identification of debris helps the problem in several ways; most important is the characterization of the debris on orbit. Currently material type is assumed for size estimation; however, with material identification a much finer categorization of orbital debris can be accomplished, allowing for a much improved model for the current and future debris environment. It also grants the ability to design shielding based on the material type of the debris, and as a side benefit, it could possibly allow traceability to an origin object.

Albedo and brightness are currently used to categorize objects. Albedo and brightness, if both are known, estimated, or assumed (in the case of albedo), can be used to calculate apparent size. This allows the classification of the object into a size category, and by assuming density it can also be classified by mass. These methods can be duplicated for radar observations, though radar observations are used almost entirely in low earth orbit (below 1000km [1], [2]), as the signal strength degrades significantly as distance increases. Spectroscopy was explored in the last 20 years as an improved method for identification of orbital objects. Up until the 1980s and 1990s it was thought that reflectance spectroscopy was too sensitive to noise for the purposes of accurate identification. Advancements in the 
Chapter 1 - Introduction and Background | 4 understanding of the sources of features, and how certain phenomena change those features opened up spectroscopy as a very useful tool [3].

\section{2 - Space Environment Effects}

Before delving into the numerical and analytical methods for identification of materials, this section gives a brief overview of space environment effects. The most common environment in which spacecraft operate is the vacuum environment. Spacecraft are constructed in an atmosphere, and operated in a vacuum environment. This change in environment gives rise to outgassing. Outgassing is the removal of diffused material from a spacecraft surface. This removal occurs due to the removal of exterior pressure, which unbalances the osmotic pressure in the material, so the absorbed material in the surface will tend to be released to the environment [4]. Outgassing can have two effects on the spacecraft's spectrum: first, the removal of these materials can change the material's spectrum, as the absorption characteristics change with the slight chemical change in the materials. Second, the materials tend to be deposited on other spacecraft surfaces which are within line of sight from the outgassing source [4]. This second factor is much harder to simulate accurately without a full spacecraft and longer exposure to vacuum. 
Chapter 1 - Introduction and Background | $\mathbf{5}$

Another significant effect in low earth orbit is atomic oxygen erosion. In the upper atmosphere, exposure to ultraviolet radiation from the sun dissociates diatomic oxygen $\left(\mathrm{O}_{2}\right)$ into atomic oxygen $(20)$ which is highly reactive. Note that in low earth orbit the interaction speed between the atomic oxygen and the spacecraft will be on the order of 7-8 km/s, making the impact energy of the particles nontrivial. This impact energy is one of the two driving forces for atomic oxygen erosion [4]. The second is the high reactionability of atomic oxygen. This leads to oxygen impacting on a surface, binding to a particle on the surface, and taking the particle with the oxygen due to its high initial momentum. Certain materials experience this phenomenon less, as the materials are less reactive with oxygen [5]. This erosion can have a significant impact on the spectrum of a material; the effect is most significant in coated materials, and less significant on materials such as support structure where the material is homogenous throughout. The erosion also changes the texture of the material, so it has the possibility to change the reflectance distribution relative to the angle of incidence and observation.

Ultraviolet radiation similarly can play a role in the degradation and alteration of a material's reflectance spectrum. Ultraviolet radiation is made up of higher-energy photons than visible light, and on impact with certain spacecraft materials can break bonds or catalyze reactions due to energy input, changing its appearance. These effects are much more pronounced in polymeric materials, 
Chapter 1 - Introduction and Background | 6 where chain-like molecules make up the material. When those chains are broken, the appearance and other aspects change more drastically [4].

The final space environment effect that can cause material appearance changes is the plasma environment. Plasma is a charged gas, more common in low earth orbit where the rarified atmosphere at that altitude is denser; it crops up around a spacecraft due to charge imbalance between parts of the spacecraft, or in the plasma itself [4]. The plasma on its own does not cause material appearance changes, but when the potential between areas on the spacecraft becomes high enough, arcing occurs. Arcing can disfigure areas on the surface, changing the reflectance spectrum.

These four effects comprise main phenomena that have an effect on a spacecraft's appearance as it weathers over time. There are a few synergistic effects associated with these - cases where the phenomena interact to produce a stronger change. Outgas deposition on a surface that is subjected to a plasma can change the deposited material [4], causing polymerization in some materials, and clouding in others. Atomic oxygen and ultraviolet radiation can produce a strong reaction as well, as the atomic oxygen can erode the surface coating or hardening, exposing more sensitive materials underneath, possibly allowing the ultraviolet radiation to affect a more reactive material. Good spacecraft design can eliminate or reduce some of these effects, making the identification process simpler. It is necessary to 
Chapter 1 - Introduction and Background | 7

measure these effects on materials, especially in identifying older spacecraft or debris.

\section{3 - Spectroscopy and Spectral Analysis}

This section covers the theoretical explanation of the combined spectra measured, and the math required to set up the problem and understand the function of the spectrometer. As mentioned in previous sections, spectroscopy $\mathrm{i}^{-}$the measurement of light magnitude relative to wavelength. This is most commonly accomplished using a diffraction grating to split the light angularly into its constituent wavelengths. It is useful to cover this functionality to provide a basis for future troubleshooting. As shown in Figure 1, a diffraction grating functions by causing constructive interference at a point corresponding to a specific wavelength, and destructive interference elsewhere. This interference arises due to slight differences in path length from the individual slots in the grating, and can be measured predictably by angular location:

$$
d \sin \theta_{m}=m
$$

where $m$ is the integer mode of interest, $\lambda$ is the wavelength in units of length, $\theta_{m}$ is the angular distance of the maxima from the centerpoint, and $d$ is the slit spacing in the diffraction grating in the same units as wavelength. Using an appropriately convex or concave mirror, and placing a distributed line of CCD pixels 


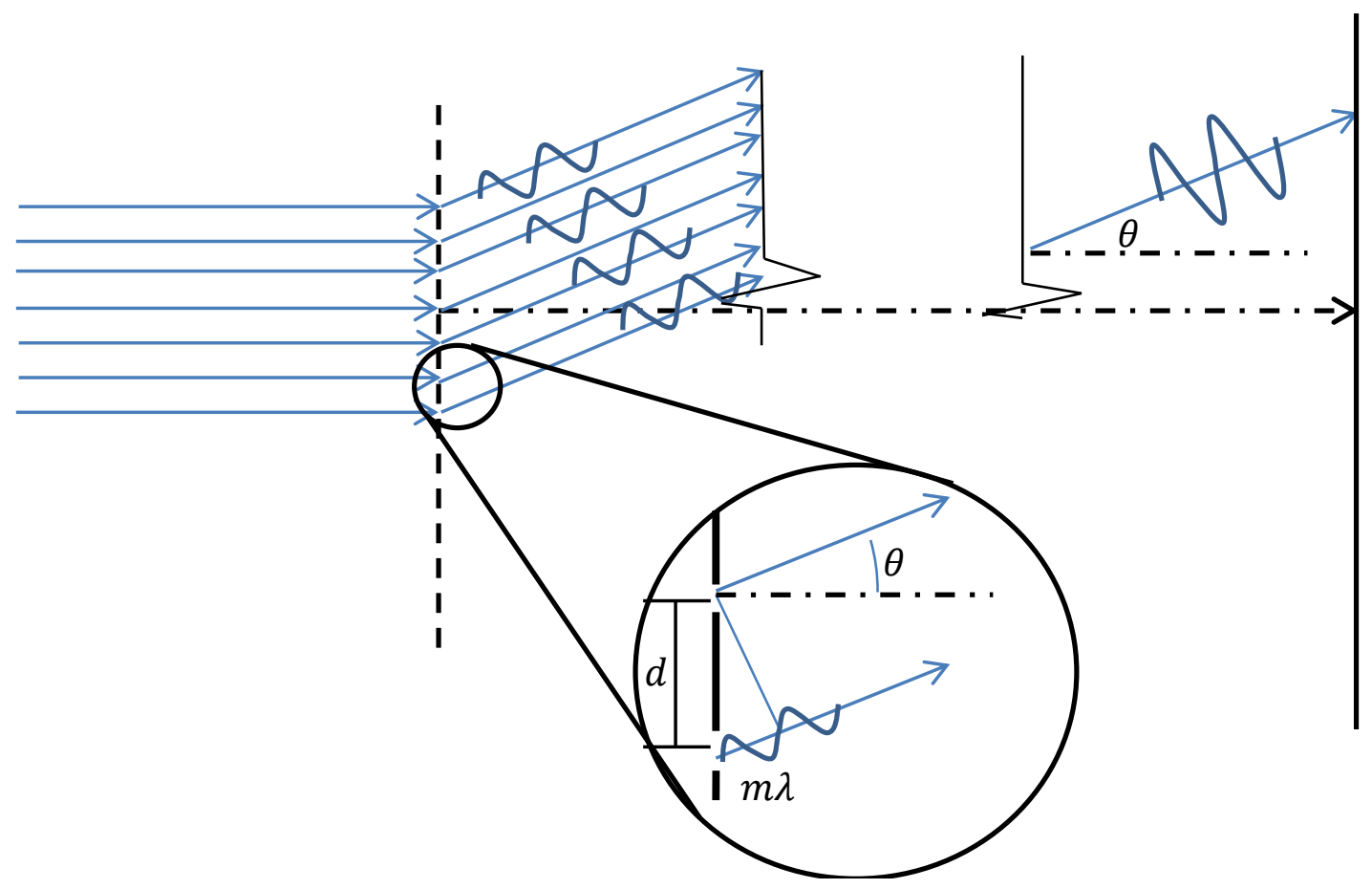

Figure 1. Diffraction Grating Diagram.

Shown above is the geometry and associated variables for a diffraction grating. For each angle $\theta$ relative to the optical axis, there is a wavelength which is constructively interfered. This property allows the diffraction grating to produce a spectrum broadening effect much like a prism, and allows its use as an integral part of the spectrometer.

at the angles $\theta_{m}$, the magnitudes read out correspond to the light intensity at each wavelength step.

An important phenomenon to note is the resolving power of a telescope or array of telescopes. This resolving power calculation shows that measurements of spacecraft are typically not readily resolvable for material identification, on a pixelby-pixel measurement, and must be treated as a point source. Resolving power is calculated based on circular aperture diffraction, and the limit to resolving power can be calculated via the Rayleigh criterion [6]: 
Chapter 1 - Introduction and Background | 9

$$
\sin \theta=1.220 \frac{\lambda}{D}
$$

where $\theta$ is the angular resolution of the optical system in radians, $\lambda$ is the wavelength of light in meters, (should be taken as an upper bound, as a larger wavelength requires a larger aperture diameter to attain the same angular resolution), and $D$ is the diameter of the telescope's aperture in meters. Due to the distance of the objects observed relative to the size, the small angle approximation can be used to simplify the criterion [6]:

$$
\frac{d}{Z}=1.220 \frac{\lambda}{D}
$$

where $z$ is the altitude of the object in meters, and $d$ is the distance between the two components to be resolved in meters. Assuming the best case scenario for observing a spacecraft directly, to find the minimum diameter of a telescope needed to observe the spacecraft components directly. Assume low earth orbit at $z=200 \mathrm{~km}$, a spacecraft with components approximately $d=0.1 \mathrm{~m}$ apart, and an upper bound on wavelength at $\lambda=1000 \mathrm{~nm}$ :

$$
D=1.220 \frac{\lambda z}{d}=2.44 m
$$

This value represents the absolute minimum size, as it represents a nearest possible object with component separation of only $10 \mathrm{~cm}$. If the object is only at the upper bound of low earth orbit, it increases the size required to a 24.4 meter telescope. This size telescope is often infeasible, so the methods used to identify 
materials cannot always involve spatial separation of materials. Also worth noting is that this is a lower bound on the telescope size requirement, ignoring atmospheric disturbances, which are often much larger disturbances to the resolution of the image.

Linear mixing is a description of the mode of combination when distinct material spectrum components are mixed into an unresolved light signal. This is the mode which occurs to produce the spectra used in this project. Shown in Figure 2, as the light reflects from the surfaces of the materials the spectra from each material adds to the total signal, if the assumption made above that the materials cannot be spatially resolved holds.

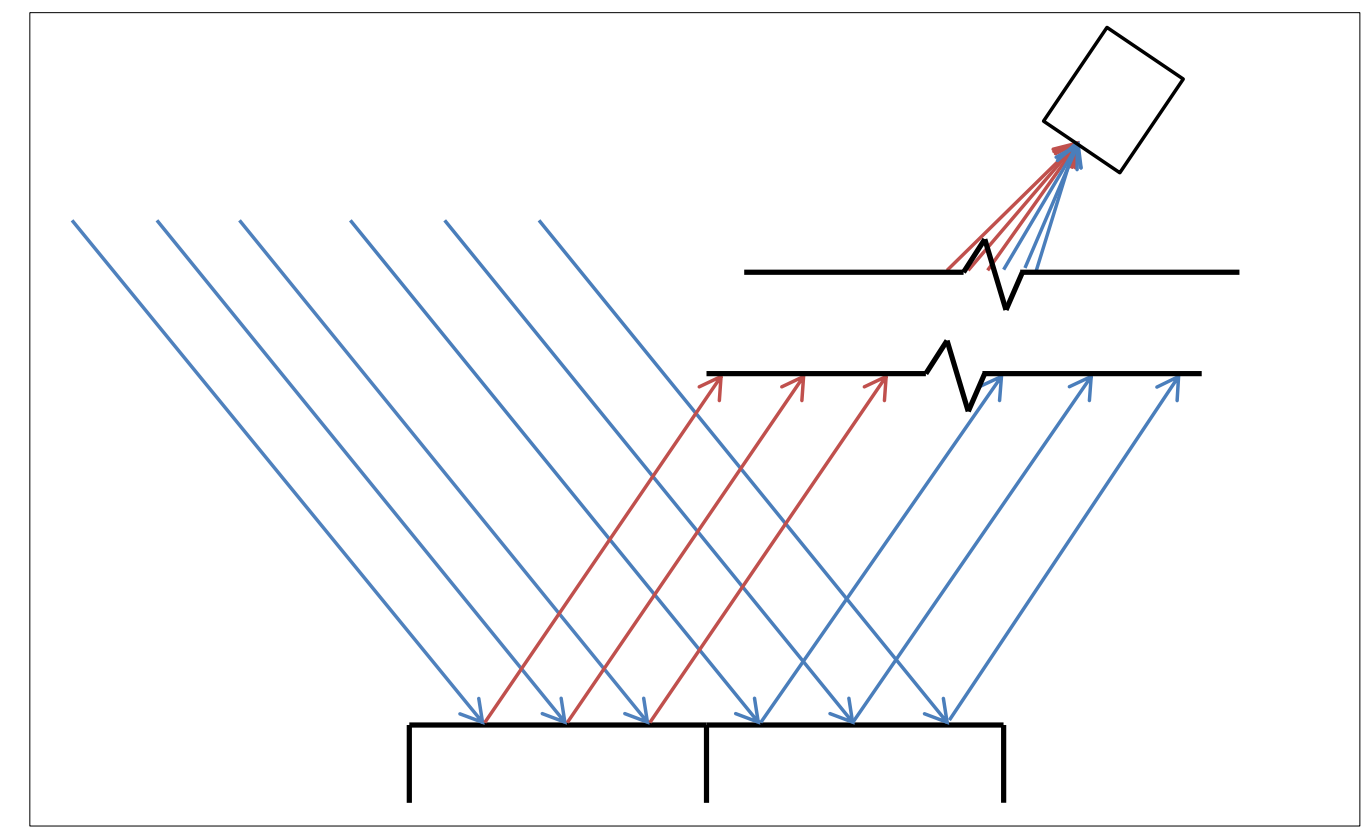

Figure 2. Linear Spectrum Mixing.

Shown above is a visual representation of linear spectrum mixing. When the individual materials cannot be resolved by the spectrometer, the spectra are linearly mixed 
This leads to spectra adding linearly according to the proportion represented on the surface of the object [7]:

$$
S_{\text {combined }}=\sum_{i=1}^{n} p_{i} S_{i}+N
$$

where $S$ is a spectrum, $i$ is an index representing the $i^{t h}$ material, $p$ is the material proportion of the full spectrum, and $N$ is noise. This method, however, ignores changes in spectra due to the orientation of both the incident light and orientation of the object. These changes are most significant on the magnitude of the spectrum, and incorporating them into the unmixing process is outside the scope of the project. The full equation defining the combined spectrum in terms of orientation [7]:

$$
S_{\text {combined }}=\sum_{i=1}^{n} p_{i} B_{i} S_{i}+N
$$

where $B_{i}$ is the orientation coefficient for the $i^{t h}$ material. Equation (6) is still an approximation, however, as the orientation can change the spectrum in more ways than changing the magnitude. An example of this is thin film coatings common on solar cells, the angle reflection plays a role in determining the location of the strong reflectance feature. The orientation coefficient, $B_{i}$, can be determined analytically using [8]:

$$
B_{i}=\frac{d L_{r}\left(\vec{\omega}_{o}\right)}{E_{I}\left(\vec{\omega}_{I}\right) \cos \theta_{I} d \vec{\omega}_{I}}
$$


where $E_{I}$ is the incident irradiance on the vector $\vec{\omega}_{I}, \theta_{I}$ is the angle between $\omega_{I}$ and the surface normal ( $\vec{N}$ in Fig. 3$)$, and $d L_{r}$ is the radiance observed along the vector $\vec{\omega}_{o}$. L can be calculated using [9]:

$$
L=\frac{\Phi}{\Omega A \cos \theta}
$$

where $\Phi$ is the total radiant flux reflected by the surface, $\Omega$ is the angle subtended by the measurement device (in this case the spectrometer probe), $A$ is the area of the surface, and $\theta$ is the angle between the surface normal $\vec{N}$ and the $\vec{\omega}_{o}$ vector. A geometric representation of the orientation of these variables is shown in Figure 3.

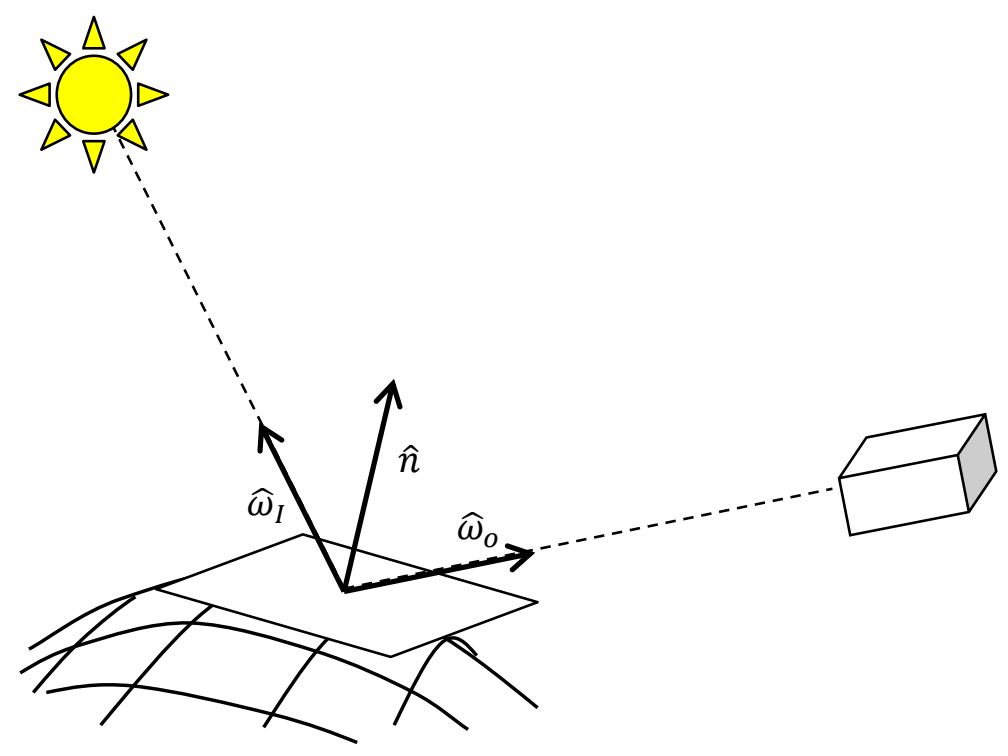

Figure 3. Bidirectional Reflectance Distribution Function (BRDF).

Shown above is a geometric representation of the variables used in calculating BRDF. $\widehat{\omega}_{o}$ is the unit vector pointing in the outgoing light direction, toward the sensor. $\widehat{\omega}_{I}$ is the unit vector in the incoming light direction, and $\hat{n}$ is the unit normal for the surface tangent plane. 
In practice, the distribution of magnitudes varies a fair amount from this ideal case. An example of this non-uniformity can be shown in Figure 4, from data presented in Ref. [10], taken from the TASAT BRDF database:

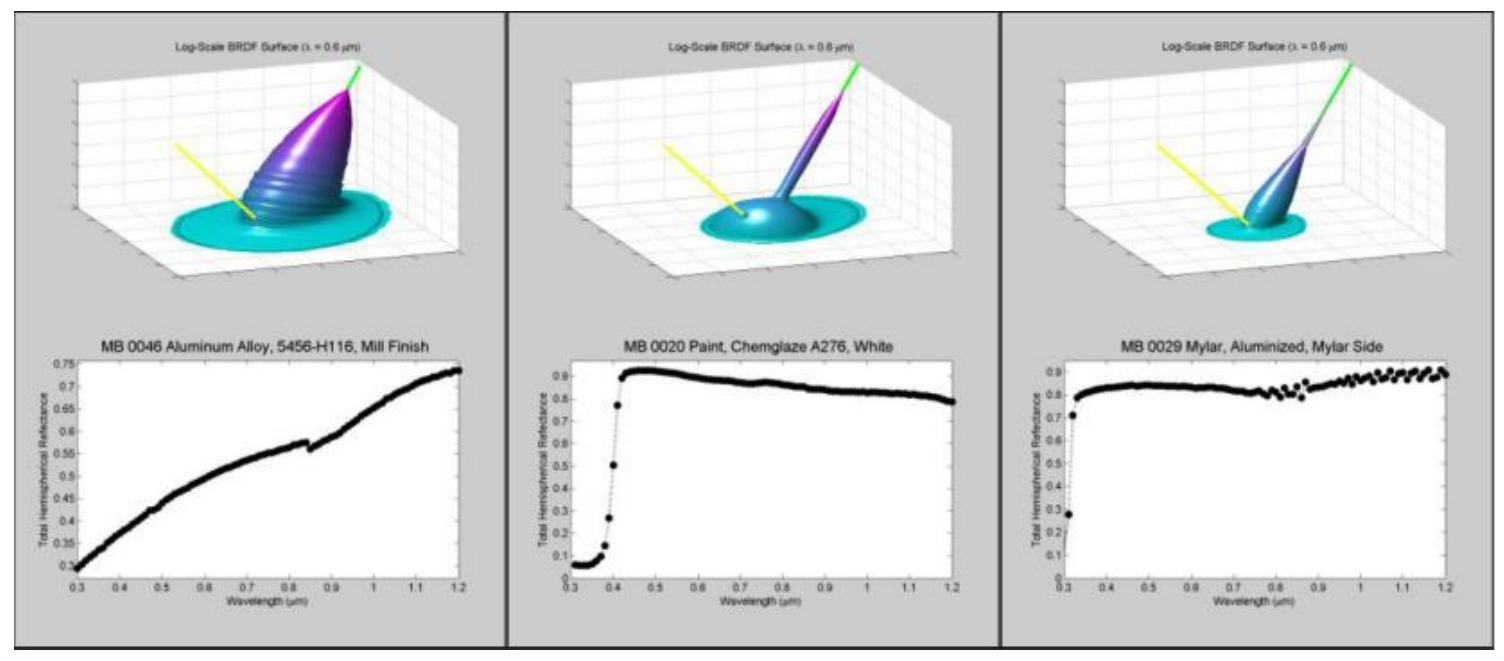

Figure 4. BRDF From TASAT Database.

Shown above is a geometric representation of the variables used in calculating BRDF. Materials from left to right: Aluminum Alloy Mill Finish, White Chemiglaze Paint, Aluminized Mylar, on the Mylar side. [10]

Figure 4 above shows a three-dimensional representation of measured BRDF values for three satellite materials in the upper panels, all taken at $\lambda=600 \mathrm{~nm}$. The yellow line shows the orientation of the incident beam of light, and the green line shows the incident light vector reflected about the surface normal. Note that the shinier materials have a much higher light emitted near this reflection axis due to the higher presence of specular reflection, where a more 'dull' surface has a more even distribution of reflection due to the higher magnitude of diffuse reflection. The 
Chapter 1 - Introduction and Background | 14

lower panels in Figure 4 show a spectrum taken of the material over the range $300 \leq \lambda \leq 1200 \mathrm{~nm}$ for comparison.

\section{4 - Previous Work}

Beginning in the late 1990s, work has been performed investigating and developing the use of spectroscopy to identify materials on orbital objects. The work of Dr. Kira Abercromby has played a large role in the investigation of spectroscopy applications for this field, with a large number of materials measured and methodology developed to take measurements of orbital objects. One of the more successful examples was the observation of object J002E3 [11], which is in a large 50-day orbit around earth. The measurement of this object's spectrum enabled the identification of the object as a NASA rocket body, specifically an Apollo upperstage, Saturn IVB. There have been other occurrences applying spectroscopy to identify materials on orbital debris [12] [13], showing that spectroscopy can be successfully used to identify materials on orbital objects.

The Tetracorder project was a program developed in the early 2000's for identifying materials based on imaging spectroscopy [14]. The goal of the Tetracorder project was to identify surface materials on the planet to aid in mapping resources using hyperspectral imaging. In this case the materials which are measured are actually separable pixel by pixel, so that identification can be 
Chapter 1 - Introduction and Background | 15

made on one material at a time. This identification was accomplished by feature matching with known materials. A series of algorithms were created which matched feature shape, position, and size with distinct features in the measured spectrum. Tetracorder was successful when the number of materials in an observation was small. However, as the number of combined spectra grows, each feature gets relatively smaller and smaller, making it harder to identify a feature compared to a variation in the spectrum due to other factors.

Another project in the early 2000's attempted spectral unmixing on spacecraft assuming a linear combination of spectra [15] [16], [17]. Their method utlized non-negative matrix factorization, and was similar to the methods developed in this thesis, though less effective at determing material composition. Applying a constrained gradient type solution to the linear unmixing problem, the constrained non-negative matrix factorization resulted in a different (but close) answer on each run with the input data. This would indicate that the method employed was not a true optimization, as the methods employed in this report are, but were attempting to approximate the optimum solution. Their methods were tested on laboratory compositions, and had difficulty with distinguishing between some materials. Ultimately, the method required a large number of iterations and best results were achieved within $90 \%$ of the correct measurements, at worst there were significant differences between the unmixed results and the originals. 
Chapter 1 - Introduction and Background | 16

A project that used tensor methods on hyperspectral imaging to do spectral identification followed from this original project, with similar results [18]. This used methods modified from the project above to do single-material, or small numbers of materials unmixing on a simulated hyperspectral image of the Hubble Space Telescope, allowing pixel-by-pixel identification. Even so, the results were close, but suffered from the same sort of problems as the original project: difficulty in distinguishing between similar materials, and some general inaccuracy.

A more recent project at AMOS showed a method for identifying orbital objects using BRDF measurements [10]. The method was developed for use identifying nanosat class objects with simulated data. The simulated scenario was successful with simple one-material to a side testing, though it did not perform well with similar materials. The model also did not perform well with non-present materials and noise.

Other work has involved using Doppler interferometry to estimate object shape and size [19], and using other characteristics of the signal received to identify the material.

There has been work on the appearance effects of space weathering on materials, typically associated with manned spaceflight, as those are among the few materials returned to earth after being flown and exposed to the space environment. An example of this is on an ISS mission, it was noted that a sample of Teflon Silver 
Chapter 1 - Introduction and Background | 17 Inconel, exposed to the AO and VUV environment appeared darker on the Teflon side of the sample [20]. It was believed to be a chemical change in the Teflon. Thermal control materials are considered to be the most important for this type of testing, as their functionality is dependent on the physical appearance of the material. If the material's reflectivity or absorptivity changes significantly, the thermal properties may stray outside of the design profile, causing issues with the thermal control on the spacecraft. This prompted the study in simulated space environment setups at the NASA Lewis Research Center (renamed to the John $\mathrm{H}$ Glenn Research Center) on several thermal control materials for use on the ISS [21]. There were changes measured, though very slight on the materials tested (on the order of \pm 0.002 - 0.07 (unitless figure, change in absorptivity, which is measured 0 1).

Finally, most work on the spectral effects of space weathering is focused on the weathering of surfaces on natural objects, such as the lunar surface [20], or on non-appearance related material degradation [21]. Interesting work on the reddening effect observed on asteroids and other objects exposed to the solar wind for extended periods of time has been carried out, and can likely be adapted to spacecraft simulations [22]. 
Chapter 1 - Introduction and Background | 18

\section{5 - Conclusion}

Spectroscopy has proven to be a valuable asset in the identification of orbital objects, both debris and spacecraft. This project has furthered that work to develop spectroscopy to provide a more streamlined identification methodology. This was accomplished by beginning the process of measuring the spectral impact of some types of space weathering, and by developing an unmixing technique to identify complex objects of more than a few combined materials. 


\section{2 - Spectral Measurements}

The measurement of spectra plays an important role in having an appropriate basis for unmixing and identification. In this section the appropriate methods for taking spectral measurements to observe the effects of space environment weathering are investigated, and the measurements are discussed. Materials for measurement were obtained from Cal Poly's space environment labs, the Cal Poly PolySat project, and a set of thermal film samples were acquired for use in testing. The other major source for materials, though not subject to the space environment testing, were a pair of CubeSats. Auburn University's AubieSat, along with Montana State University's Explorer-1 satellite, renamed the William A. Hiscock Radiation Belt Explorer (HRBE) after launch to honor Montana's first Space Grant Director. Other spectral measurements were made on cubesat components and completed busses which were made available over the course of testing.

The lab measured spectra presented in this report were measured using a commercial Analytical Spectral Devices FR spectrometer. The field spectrometer has a range from 0.3 to 2.5 microns with resolving power of approximately 200 (corresponding to a bandwidth of $10 \mathrm{~nm}$ at two $\mu \mathrm{m}$ ) and 717 channels. The system only needs 210 channels in order to obtain the desired bandwidth, so using 717 channels is over-sampling the data, which results in the advantageous 
lessened degradation of spectral resolution. A fiber optic cable is held in place by an optic pistol grip, which is oriented approximately perpendicular to the surface of the sample so that the shadow of the probe does not disrupt the spectral response. This cable is fed into the ASD FieldSpec spectrometer where the light is dispersed over three spectrometers contained within, each covering a different band of wavelengths. The data is fed back to a computer, where it can be viewed and recorded. This data is reduced and post-processed using a program called ViewSpec Pro. Within this program, artificial features due to the jump in bandpasses between the spectrometers can be removed as well as the creation of ASCII text files for use in other programs. Figure 5 depicts the bandpasses for each of the three spectrometers: seven, 11 , and $11 \mathrm{~nm}$ bandpass respectively [3].

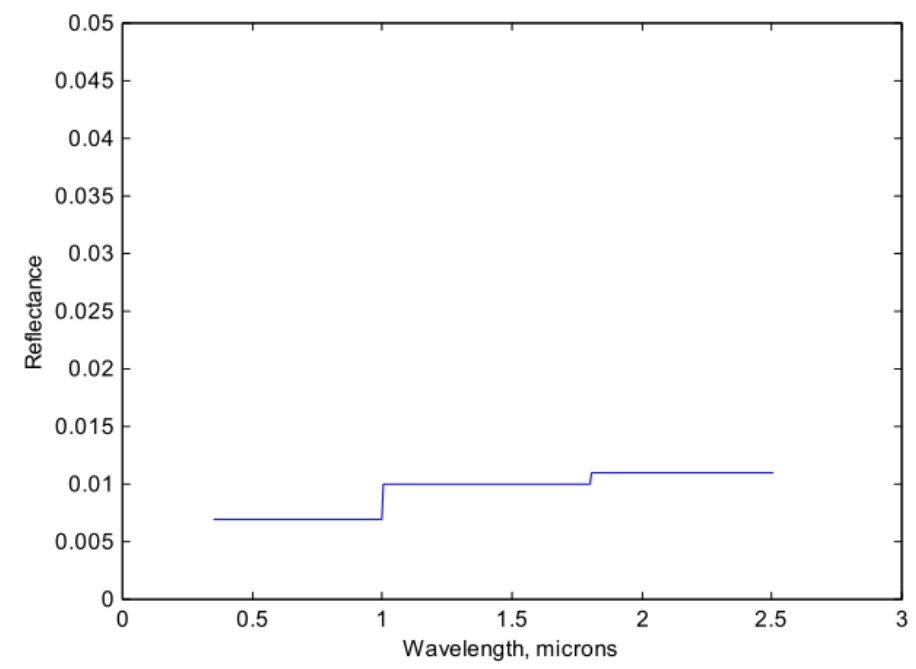

Figure 5. ASD FR Spectrometer Bandpass.

Shown above is the bandpass for the ASD FR Spectrometer used in taking the spectral measurements shown in this report. 
The Analytical Spectral Devices, Inc FSP 350-2500P was used to make the measurements described in this section and elsewhere, unless otherwise noted.

\section{1 - Standard Procedure and CubeSat Measurements}

The typical setup for taking spectral measurements consists of a tungsten light source, a spectralon sample used as a white reference, a probe with a handle, a fiber optic cable, the ASD FieldSpec Spectrometer itself, and a laptop loaded with the appropriate software for data collection and post-processing. These components are set up as shown in Figure 6 below.

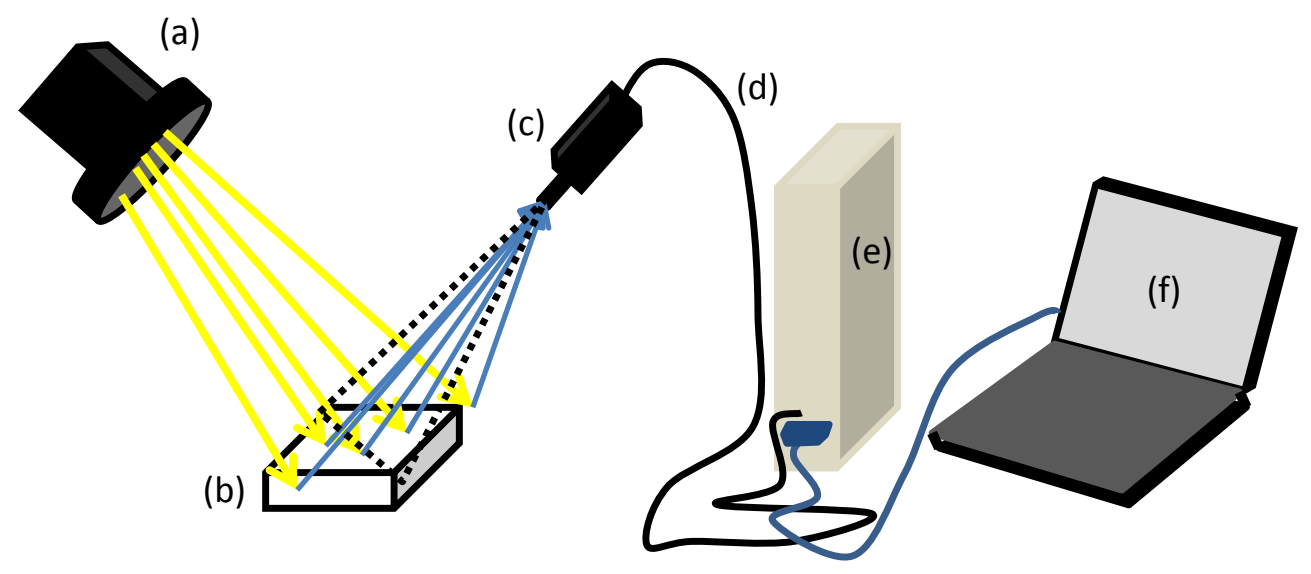

Figure 6. Typical Configuration for ASD FieldSpec Spectrometer.

Components as follows: (a) Tungsten Light Source, (b) Spectralon White Reference or Sample, (c)Fiber Optic Probe, (d) Fiber Optic Cable, (e)ASD FieldSpec Spectrometer, (f) Laptop Computer 
The important first step in setup is warming up the tungsten lamp and the spectrometer. In order for the lamp to get to uniform brightness it takes about 10 - 15 minutes, while the spectrometer takes about 20 minutes. If this warmup is not completed, the magnitude of the spectral measurements will be incompatible. While the lamp and spectrometer are warming, set up the rest of the equipment as in Figure 6 and turn on the equipment. Adjust the configuration of the spectrometer in the $\mathrm{RS}^{3}$ software (the software preloaded on the laptop for reading spectral data from the spectrometer, proprietary from ASD). To calibrate the spectrometer to the light conditions run an optimization with the optical probe receiving light from the white reference which the light source is illuminating. Before beginning data collection, it is important to note the position of the white reference relative to the fiber optic probe. For consistency, it is important to maintain this distance when a material sample is switched out for the white reference.

Next, take a white reference measurement to ensure that the reflectance signal received from the set-up is the intended one. Then replace the spectralon with the sample to be measured. For this thesis, spectra samples were typically taken in sets of 3 , to prevent any motion or other anomalies from interfering with the veracity of the measurements. Intermittently during measurements, it is good to check the white reference to make sure the calibration has not degraded. The time between recalibrations required for this thesis was not 
consistent, though the average time was between 5 and 20 minutes. Once data collection has been completed, there are two important post-processing steps that are required: bandpass correction and ascii export. As mentioned earlier, a total of three different spectrometers are contained within the ASD FieldSpec, each covering a different band of wavelengths. To correct any inconsistencies due to the differences between these spectrometers splice correction is used on the data, to appropriately rescale the three independent data sets. This is done with the central region spectrometer as the basis, as it is the most stable of the three between measurements. Ascii export then exports the file as a text file, with a header containing the desired information about the spectra, including the material type (if entered), absolute or relative reflectance mode, and calibration information.

For most materials absolute reflectance is ideal, but when using a nonspectralon white reference material (such as a mirror for highly specular measurements) it is useful to use relative reflectance. This is, in most cases, reverted to absolute reflectance by dividing out the white reference spectrum, as is the case for measurements presented in this report which were taken as relative reflectance.

Due to the large number of spectra measured, only select spectra are shown here, all spectra taken are shown in Appendix A. The full list of materials 
measured in the lab setting (no space environment effects) is shown below in Table 1.

Table 1. List of materials for spectral measurements.

Shown below is a complete list of materials on which spectral measurements were taken. The majority of these materials are found on cubesats and fall within one of 3 categories: Circuit board, Aluminum Frame, and Solar cells

\begin{tabular}{|c|c|c|c|}
\hline Material & Source & Material & Source \\
\hline Solar Cell (Type 1, make unknown) & AubieSat & Uncoated Aluminum & Cubesat 1 \\
\hline Solar Cell (Type 2, make unknown) & AubieSat & $\begin{array}{l}\text { Solar Cell (Type 5, make } \\
\text { unknown) }\end{array}$ & Cubesat 1 \\
\hline Coated Green Circuit Board & AubieSat & Kapton Tape & Cubesat 1 \\
\hline Uncoated Green Circuit Board & AubieSat & Antenna (material unknown) & Cubesat 2 \\
\hline Solar Cell (Type 3, make unknown) & HRBE & Uncoated Aluminum Frame & Cubesat 2 \\
\hline Red Wire with Kapton Tape & HRBE & Uncoated Circuit Board & Cubesat 2 \\
\hline Red Wire & HRBE & Circuit Board & Cubesat 3 \\
\hline Green Circuit Board & HRBE & $\begin{array}{l}\text { Solar Cell (Type 6, make } \\
\text { unknown) }\end{array}$ & Cubesat 3 \\
\hline White Circuit Board & HRBE & Antenna (material unknown) & Cubesat 3 \\
\hline Black Anodized Aluminum & HRBE & Aluminum Frame & Cubesat 3 \\
\hline Grey Anodized Aluminum & HRBE & Teflon x Silver (Inconel) & Sample \\
\hline Antenna (material unknown) & HRBE & $\begin{array}{l}\text { Aluminum x 1mm 100XC } \\
\text { Kapton }\end{array}$ & Sample \\
\hline Uncoated Aluminum Frame & PolySat & $\begin{array}{l}\text { Germanium x 100CB Black } \\
\text { Kapton }\end{array}$ & Sample \\
\hline Black coated Circuit Board & PolySat & 2mm Kapton x Aluminum & Sample \\
\hline Solar Cell (Type 4, TRMM) & PolySat & Aluminum $\times 2$ mil Kapton & Sample \\
\hline Kapton Tape / Film & PolySat & Aluminum Beta Cloth & Sample \\
\hline Green Circuit Board & Cubesat 1 & $\begin{array}{l}\text { Solar Cell (Type 7, make } \\
\text { unknown) }\end{array}$ & Sample \\
\hline Anodized Aluminum & Cubesat 1 & $\begin{array}{l}\text { Solar Cell (TRMM- } \\
\text { PLBBSA0510) }\end{array}$ & Sample \\
\hline
\end{tabular}

The discussion of the results of the non-vacuum environment measurements will primarily focus on the similarities and differences between 
the various cubesat materials, possible use in identification, and implications. Note that where specific model information of solar cells was not provided by the owners of the satellites, the materials are categorized into 'types'.

Beginning with solar cells we have roughly four variations, with significant differences between the orientations and surface characteristics. Figure 7 illustrates the typical characteristics of the TRMM - PLBBSA0510 solar cells, provided for spectroscopic study by the Cal Poly CubeSat program. Two features that are common to most solar cells appear in this spectrum.

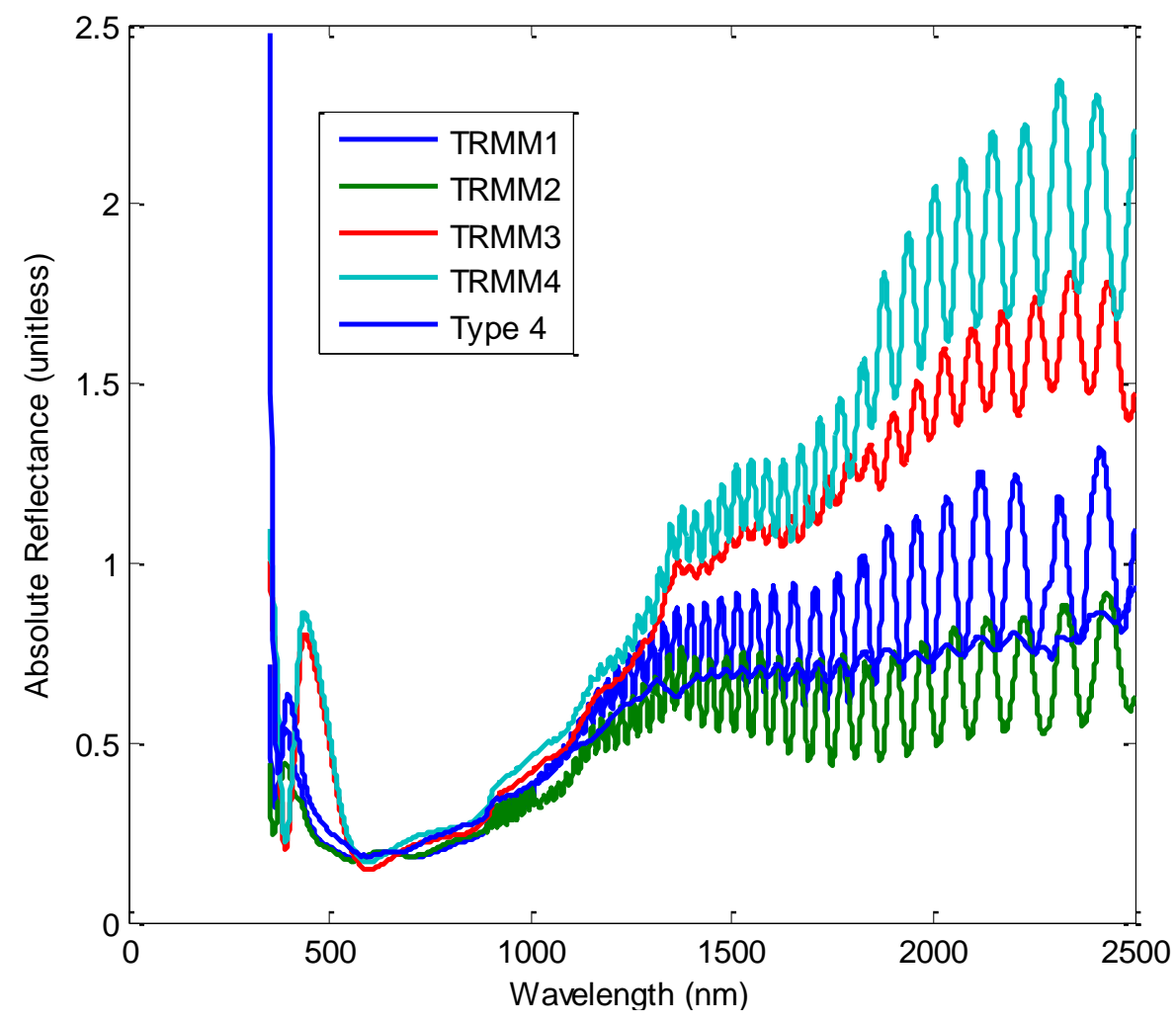

Figure 7. Solar Cell type TRMM - PLBBSA0510.

Shown above are spectra from the solar cell TRMM-PLBBSA0510, major differences come from two sources, orientation, and thin-film thickness. Note that the type 4 solar cell from the polysat lab is of the same make. 
First, the relatively high reflection peak near the lower end of the visible range, this is due to the thin film coating that is used to increase the transmission percentage in the visible region via thin film interference. Important to note, the position of this peak is highly dependent on the angle of observation and the thickness of the thin film.

The second feature common to most solar cells is an increase in the reflectivity in the near-infrared region. Throughout the visible region, the solar cell is designed to absorb as much of that light as possible, so past the visible regime the cell will begin to reflect the light.. The cell is designed this way due to the peak of the solar spectrum, which occurs between 480 and $520 \mathrm{~nm}$, depending on temperature and other factors [23]). The sinusoidal feature beginning at a wavelength of approximately $900 \mathrm{~nm}$ in is believed to be due to irregularities in the solar cell surface.

Figure 8 shows the next category of solar cells measured, found on solar cell type 2, 5, and 6 (refer to Table 1) used on AubieSat and two other example cubesats (Cubesat 1 and 3). These are categorized together based on several features, a peak at $\lambda=890 \mathrm{~nm}$, near the end of the transmission region, and the location of a relatively smooth 'hump' in the spectrum between $\lambda=1750 \mathrm{~nm}$ and the common absorption feature at $\lambda=2300 \mathrm{~nm}$, which is the last common feature between the 3 spectra shown. A point that will be discussed later in this section 


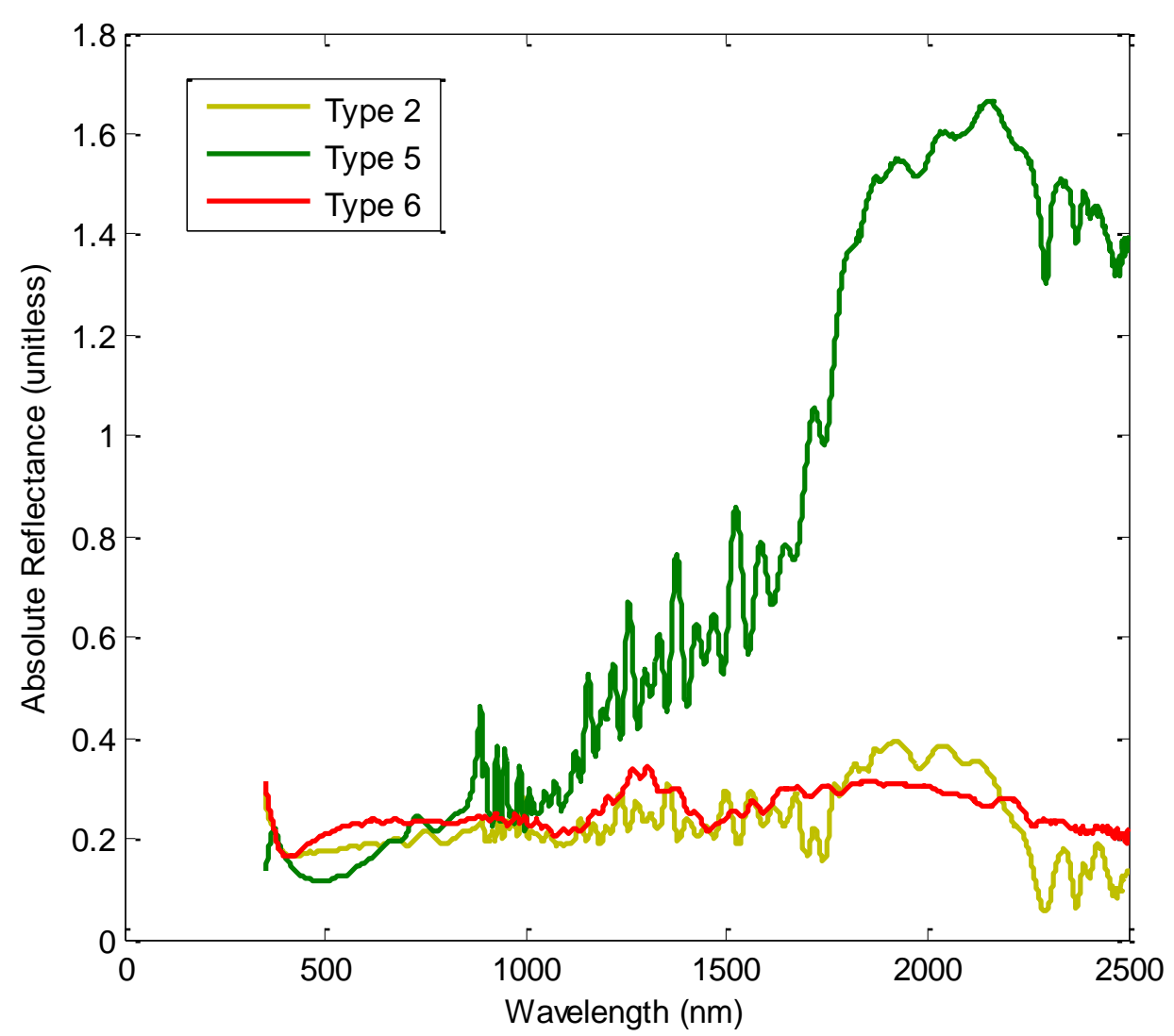

Figure 8. Spectra of the second category of solar cell, members from AubieSat and CubeSats 1 and 3.

Shown above are several spectra which have similar features, and are likely similar in composition.

is the significant difference orientation and surface characteristics play in determining the shape of the spectrum measured.

Shown in Error! Reference source not found., the third category noted only has one member, but clearly demonstrates the features that identify solar cell spectra. Unfortunately, the surface characteristics of this particular set of 
photovoltaic cells were not noted, so a comparison between this and the other

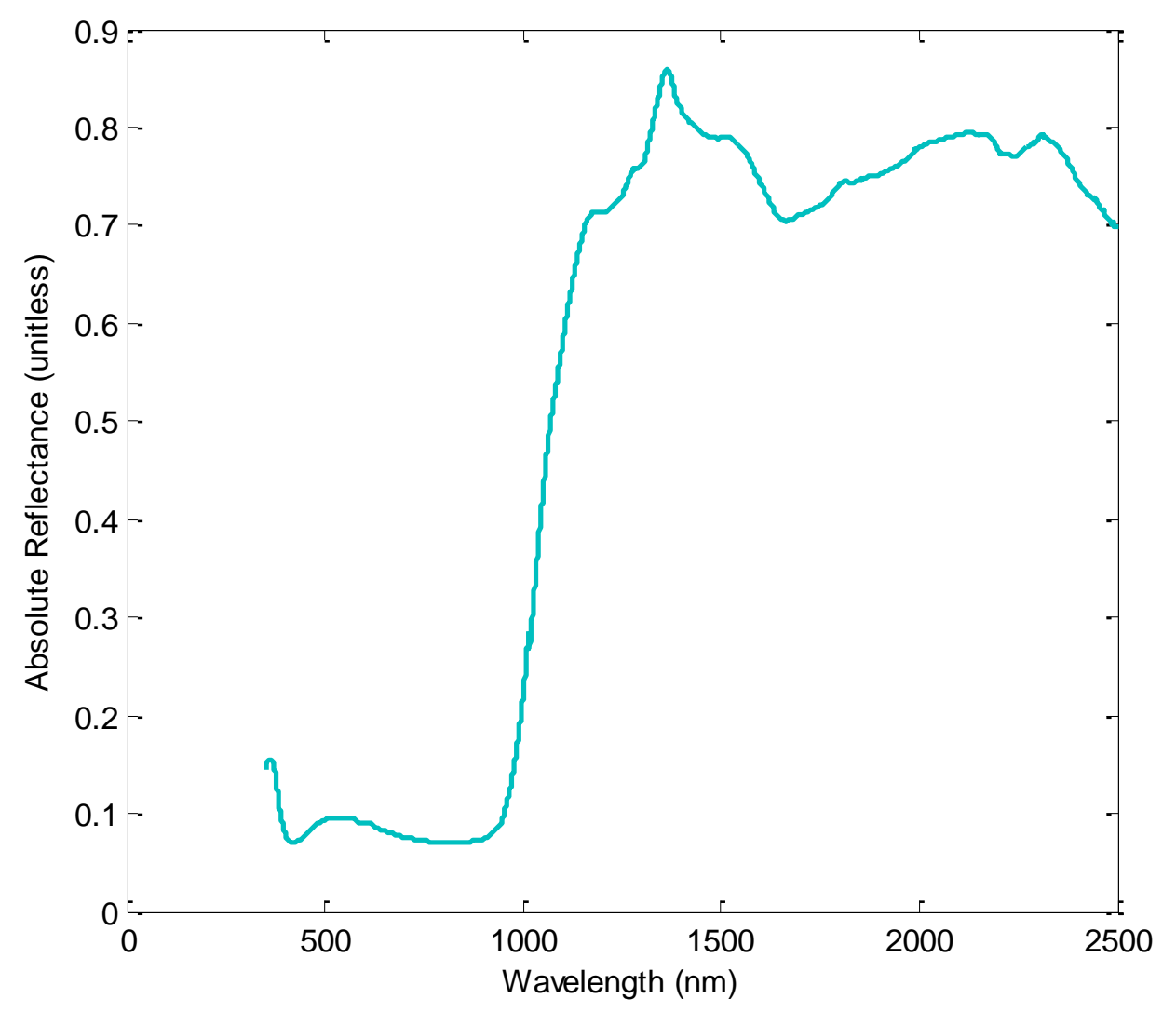

Figure 9. Solar cell, type 7 (make unknown).

Shown above is the cleanest solar cell measurement taken in the project, which clearly demonstrates the two most common features in solar cell spectra.

cells, which displayed the

sinusoidal feature over the majority of the spectrum could not be made. However, the low reflectance through the visible regime, the high reflectance elsewhere, and the peak on the lower end of the visible signifying the thin-film 
coating present on most solar cells are all demonstrated quite clearly in this spectrum.

The remaining two measurements, from HRBE and AubieSat, are fairly noisy, making identifying features difficult. The only major identifying feature is the shape similarity to the TRMM type photovoltaic cell shown in the thicker line weight in Figure 10 below:

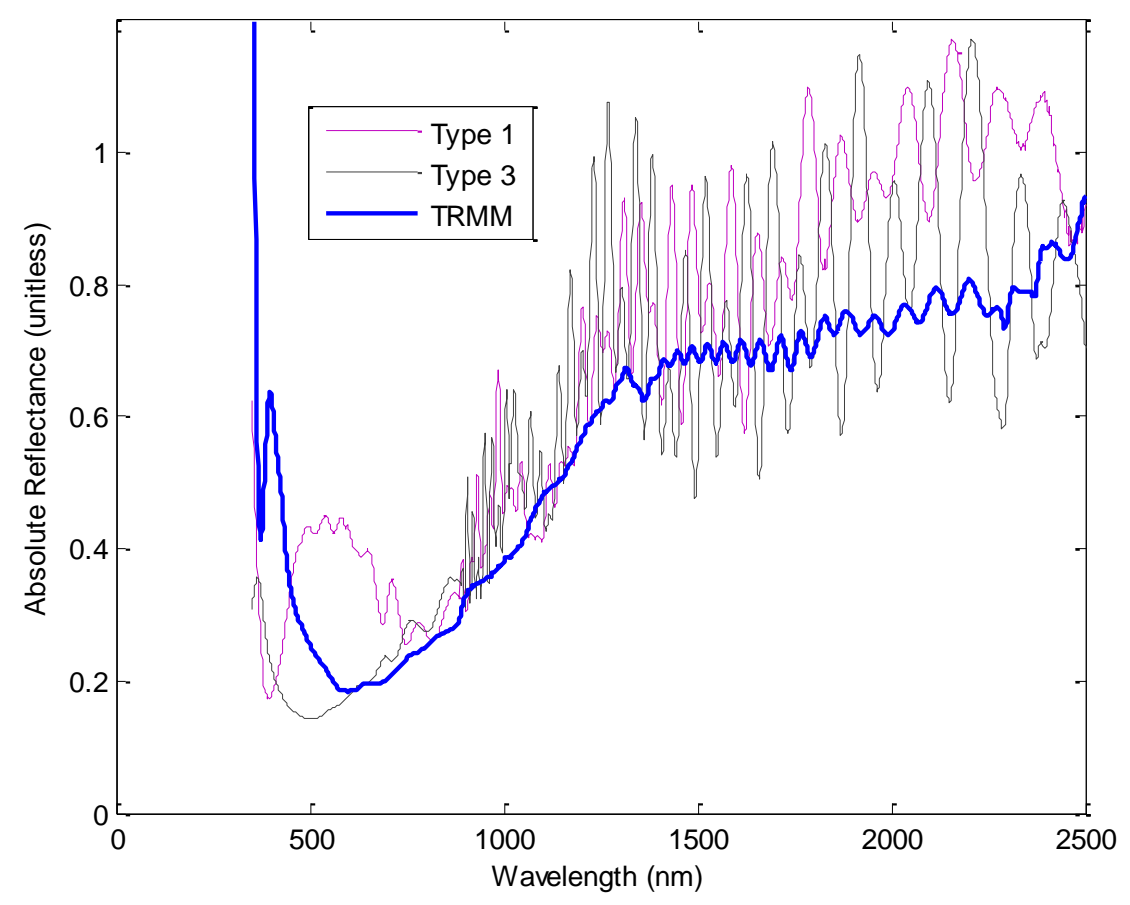

Figure 10. Comparison of solar cell TRMM-PLBBSA0510 to measurement HRBE and AubieSat solar cells.

Including both of these types, both the 'messy' measurements with significant sinusoidal features and the more smoothed measurement of TRMM provides a better basis for identification, as the true spectrum may be a linear combination of both, when measured.

The last measurement to be discussed in this section is a simple orientation-considered spectral measurement made of the TRMM photovoltaic cell. These measurements were made using a compass and straight edge to 
estimate the angle from surface normal to the fiber optic probe. The major source of error is perpendicular non-alignment, measurements not along the incident-principle reflection plane.
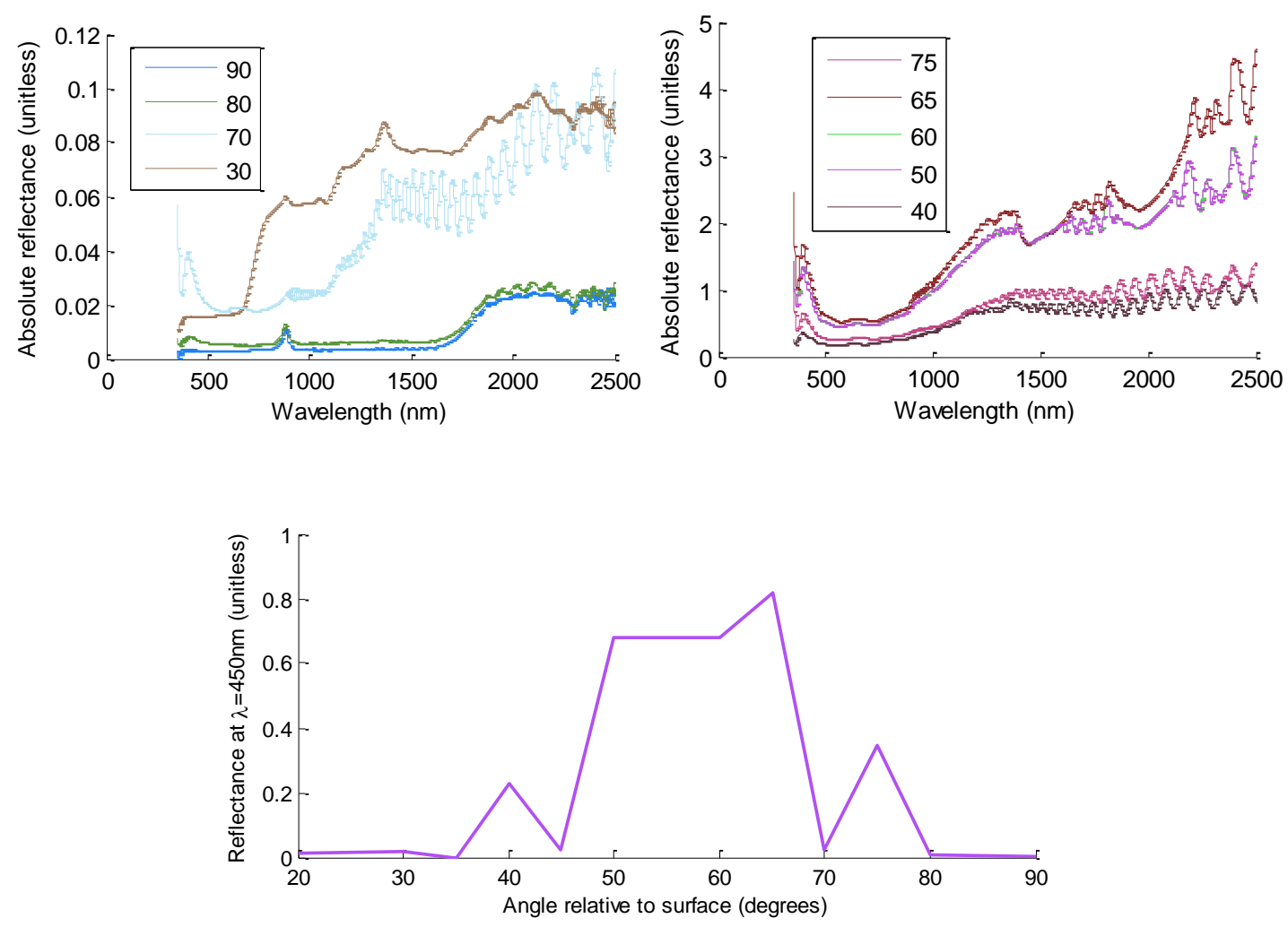

Figure 11. Directional measurement of Solar Cell TRMM spectra.

Shown above are 3 representations of the direction measurements performed. Incident light was at roughly $55^{\circ}$. (a) shows the high-reflectivity spectra at various angles (b) shows the low-reflectivity spectra at various angles and (c) shows the magnitude relative to angle. The legends for (a) and (b) have units of degrees.

Two interesting features to note, above the principle reflection axis the sinusoidal feature is less prevalent. The other interesting feature is the variation in overall curve shape depending on the angle at which the spectrum is taken. 
The second major cubesat component that appears on the exterior of the spacecraft measured is the circuit board, which is quite predictable in its appearance. It is important to note, the circuit board measurements have been normalized using an automatic normalization program written to normalize to the cleanest portion of the graph for a better comparison. Most of those measured have a very consistent set of features, and are likely the same material, despite being on separate cubesats. Figure 12 shows 4 spectra from various CubeSat circuit boards, all green, with one coated.

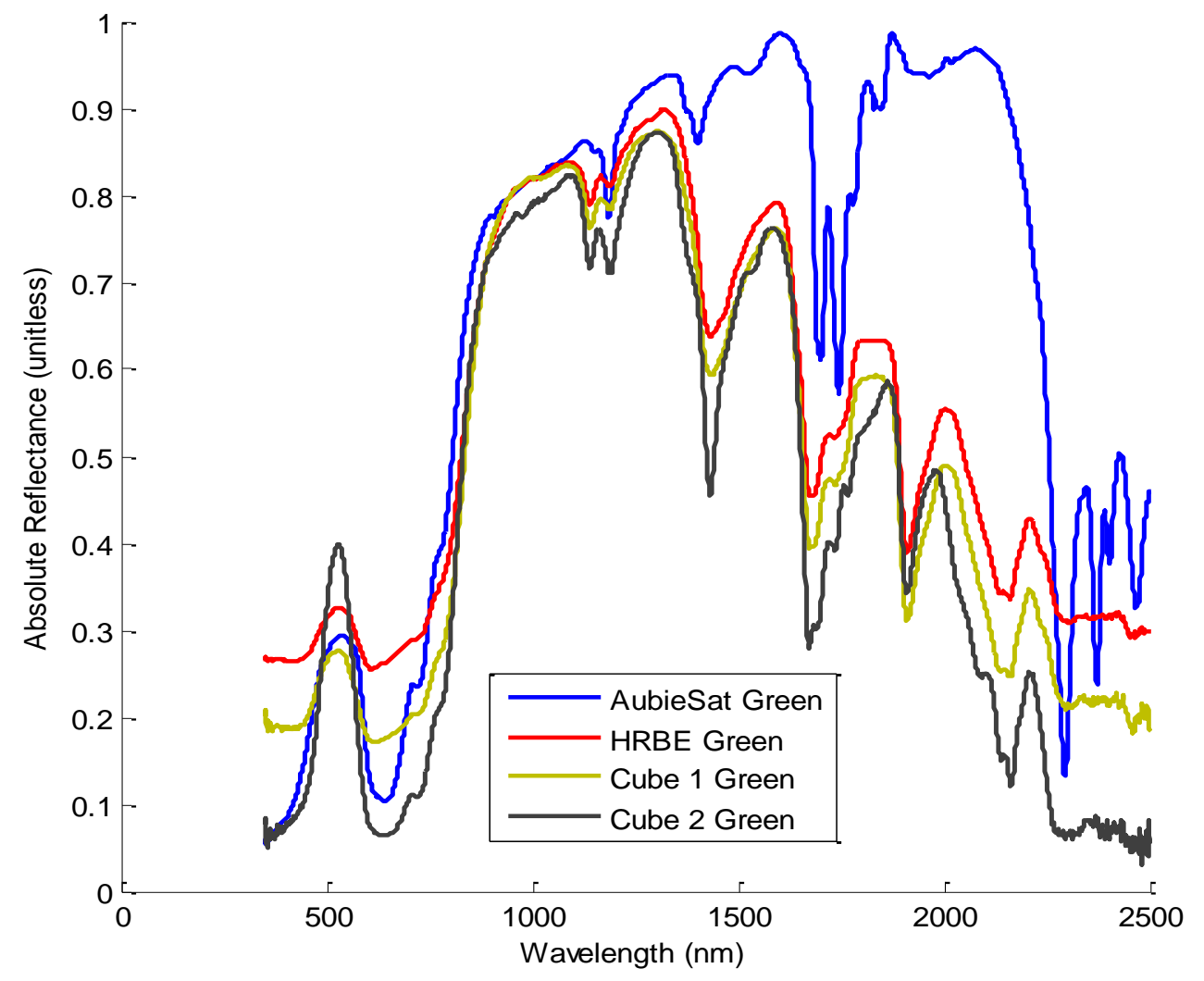


Figure 12. CubeSat green circuit board comparison.

Shown above are 4 circuit board spectra from as many CubeSats. The blue spectrum bears several similar features, but has a surface coating that changes its appearance significantly.

The similarities between the three uncoated circuit boards (red, yellow, and black in Figure 12 above) are fairly apparent. Because of this, it is likely they are the same type of circuit board. The fourth (blue in Figure 12 above) shows several of the same features, significantly muted, and modified in some cases. Interestingly, when compared with an uncoated green circuit board on the same spacecraft these features were not present, as shown in Figure 13 below. 


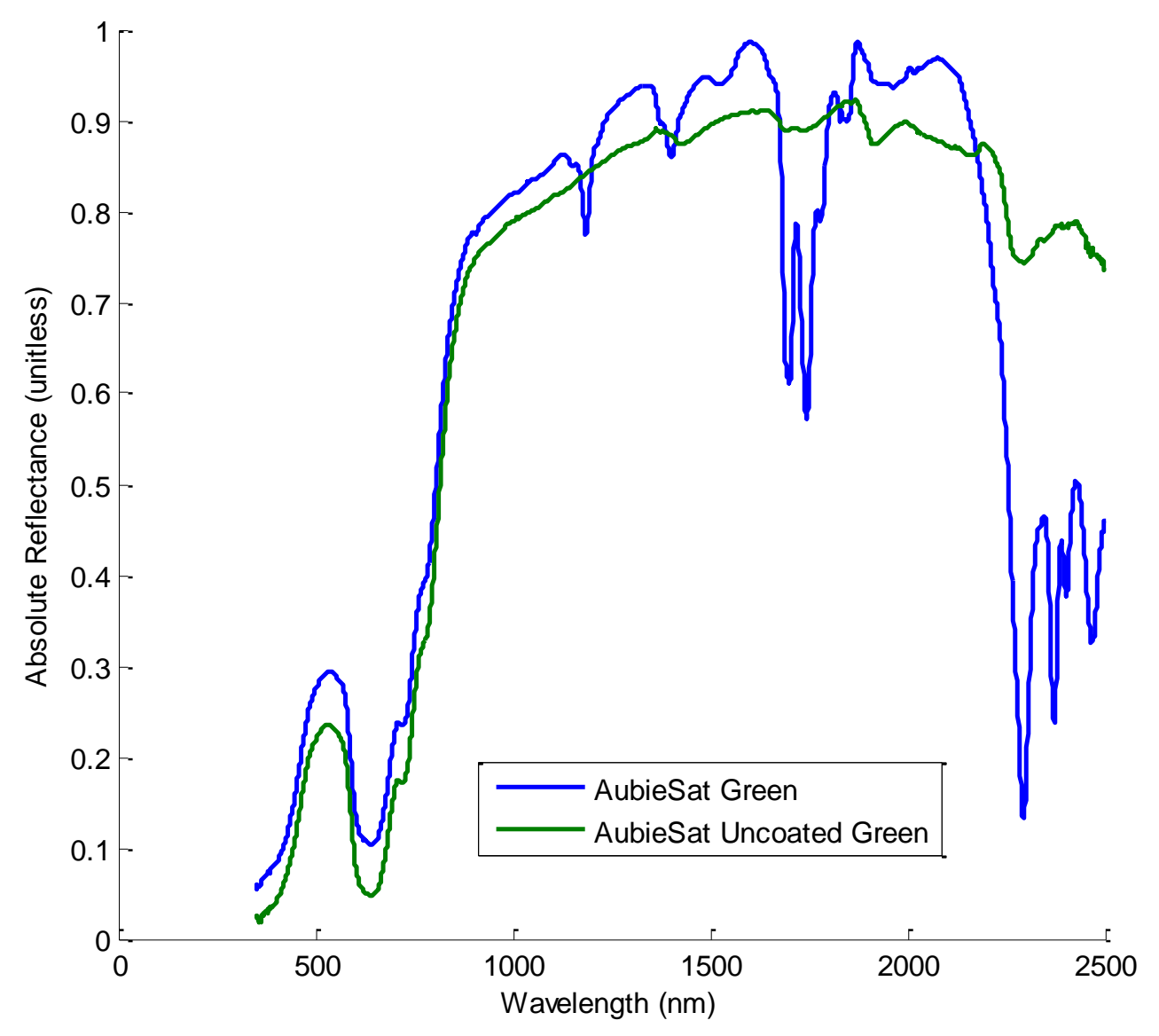

Figure 13. AubieSat coated / uncoated green circuit board comparison.

Comparing the uncoated (green above) and coated (blue above) show that the source of the absorption features is likely the coating used.

This provides an important piece of information in sourcing the spectrum, as the major features observed are due to $\mathrm{C}-\mathrm{H}$ absorption and water absorption, which are more present in the spectrum of the epoxy coated and the three other spectra shown in Figure 12. In the three spectra shown in Figure 12, the 
presence of the $\mathrm{C}-\mathrm{H}$ and water features are likely due to an organic paint used to coat the material, or other epoxy or resin used to coat the board.

The last important material to cover for CubeSats is the aluminum frame. Aluminum is fairly predictable, with a consistent absorption feature at approximately $\lambda=800 \mathrm{~nm}$. This feature is obscured with some coatings, or anodization. Three distinct variations were measured, uncoated, anodized, and black anodized. The first is shown below in Figure 14, uncoated aluminum frame from both the PolySat lab (cyan, red, and magenta) and CubeSat 2 (black). These measurements are also scaled for comparison. An interesting feature to note is in the Cube 2 anodized spectrum. This spectrum possesses the $800 \mathrm{~nm}$ feature, although more weakly than the PolySat spectra. All four frames posess two additional features (at $1370 \mathrm{~nm}$ and $2140 \mathrm{~nm}$, respectively) again with the Cube 2 spectra weaker than the PolySat spectra. This leads to the conclusion that this aluminum has a different form of anodization or coating than is seen below in Figure 15.

Figure 15 shows a comparison between three different anodized aluminum frames (from MSU's HRBE (green), CubeSat 1 (yellow), and CubeSat 3 


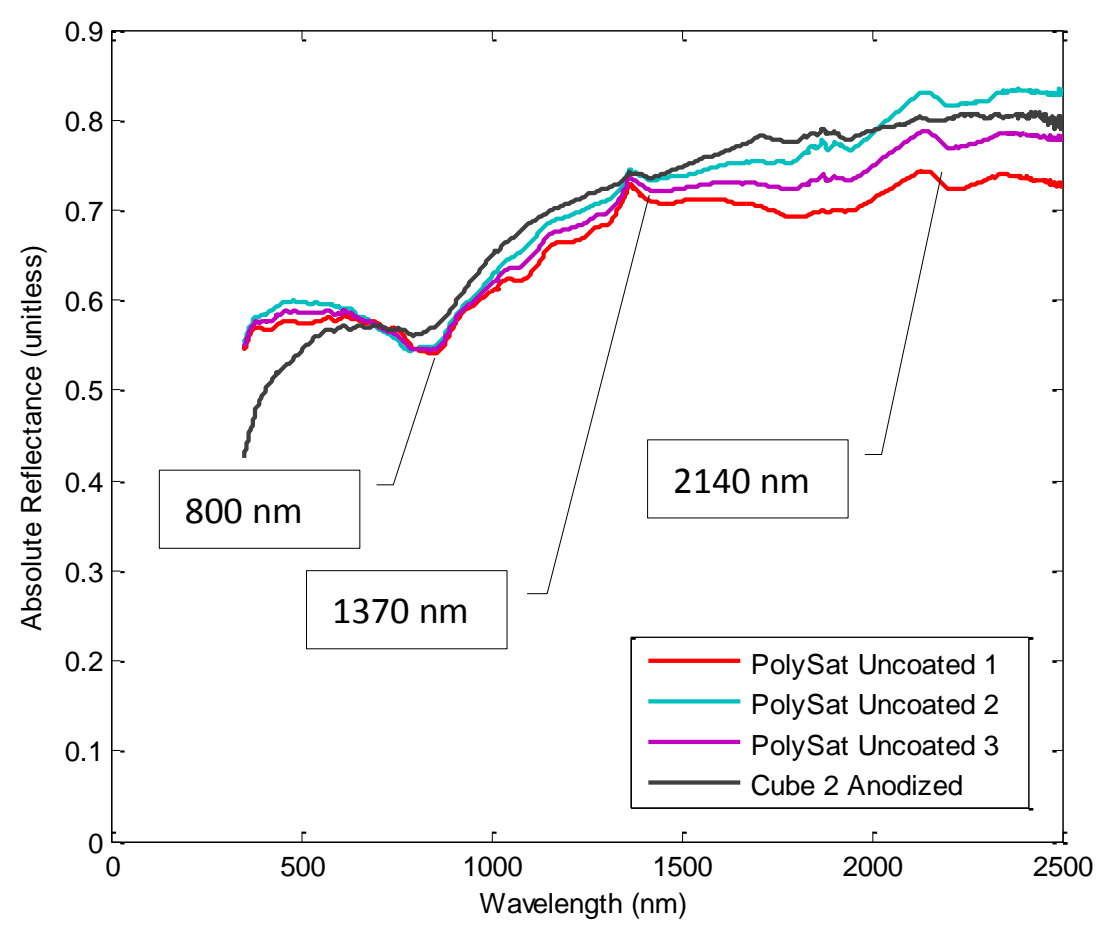

Figure 14. PolySat, and CubeSat 2 aluminum frame.

Shown above are four spectra from uncoated aluminum frames, showing the 800nm feature.

(blue)). Shown for reference is a rescaled spectrum of uncoated aluminum taken on the PolySat aluminum frame. Figure 15 shows when aluminum is anodized the feature at $800 \mathrm{~nm}$ is reduced or removed entirely. The feature at $1370 \mathrm{~nm}$ remains strong, however. The other significant difference is the addition of a positive slope outside of the visible region. This is most likely due to the process of anodizing. 


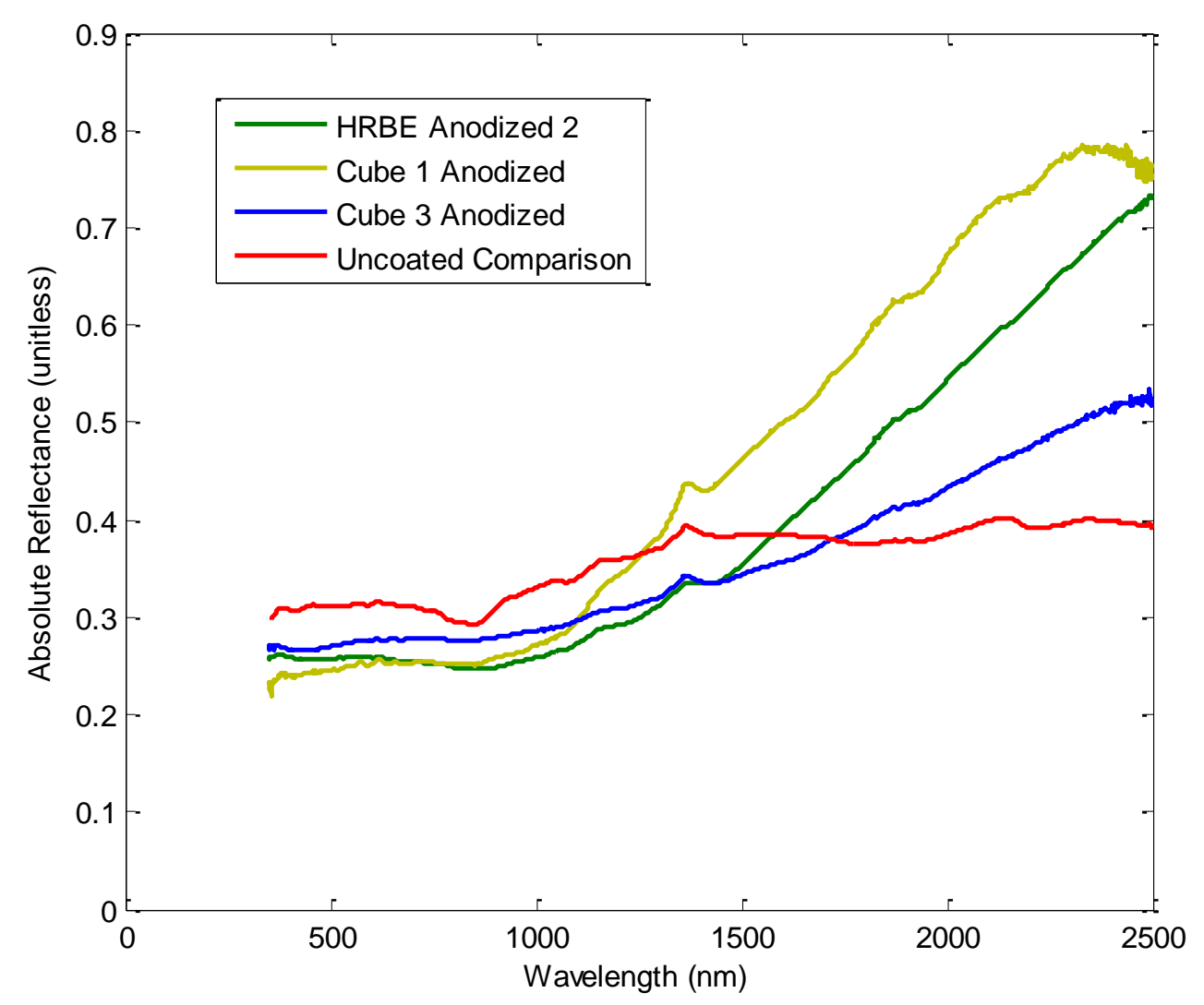

Figure 15. Comparison of anodized aluminum frames.

Shown above is a comparison of three anodized aluminum frames (yellow, green, and blue) and an uncoated aluminum frame (red)

\section{2 - Outgas Procedure and Measurements}

The outgassing effects are one of the primary concerns for developing methodology for measuring the space weathering effects on spectra. As all space environment effects take place in a vacuum, outgassing is an inevitable side effect. Given this, it's important to determine whether or not outgassing plays a significant role in changing the material spectrum, and if it does, what the 
Chapter 2 - Spectral Measurements | 37

duration of these effects are after returning the sample to the atmosphere. If the effects are short-lived, then it is important to keep the sample in the vacuum chamber when spectral data is taken. Otherwise, the samples can be measured in a dark-room facility separate from the vacuum chamber. The first set of outgas data was taken in a retrofitted Veeco Model 747 deposition chamber, which uses a roughly $50 \mathrm{~cm}$ diameter glass bell jar as the chamber wall. The chamber wall allows the measurement of material spectra while the sample is still under vacuum. The significant difference in procedure for measurements in the vacuum chamber is a difference in setup. Each sample must be lined up with the light source in such a way as to ensure an equal distance from the light source, and a similar angle and thickness passing through the glass bell jar between the sample and the white reference. This was accomplished by placing the sample and white reference equally spaced from the center of the chamber, as shown in Error! Reference source not found..

The setup has the limitation of only being able to take measurements on one sample at a time, for two reasons: keeping the attenuation from the bell jar similar between the white reference and sample, and the critical angle of the fiber optic probe. The fiber optic probe has a roughly $20^{\circ}$ critical angle, allowing input within that cone. To maintain spatial separation between the sample and the white reference it is necessary to have one sample in the vacuum chamber at a time, along with the spectralon white reference. Using the glass bell jar 


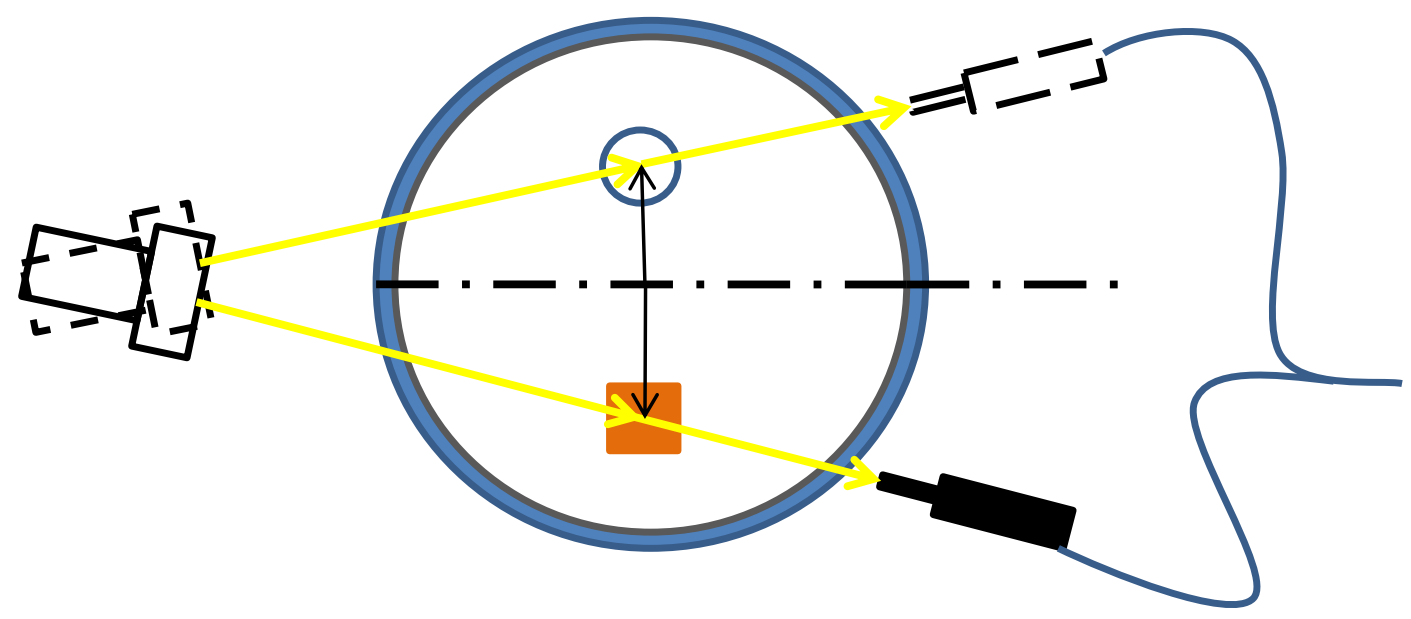

Figure 1. Vacuum chamber setup for outgas measurements taken in vacuum.

Shown above is the setup used for taking measurements in the Veeco 747. The important part is the equidistance of each sample from the centerpoint of the chamber.

chamber (Veeco 747), begin by taking a white reference and spectrum of the material before exposure to the vacuum. Evacuate the chamber to a medium vacuum, about $10 \mathrm{mTorr}$, without using the cryopump. After a period of two days the samples are sufficiently outgassed to retake spectra. Once the spectra are measured with the chamber evacuated, vent the chamber and remove the samples. This procedure can then be repeated for any other material samples to be tested.

It is important to maintain a consistent orientation for taking spectral measurements between the initial pre-outgas measurement and the post-outgas measurement. With highly specular materials (such as thermal blankets) maintaining this orientation similarity is difficult. The BRDF for these materials 
is very concentrated around the principle reflection axis, with a quick sharp drop off-axis, making acquiring a good measurement and maintaining the same orientation difficult. To minimize this source of error from orientation misalignment, a system was employed using a wet-erase pen to mark the position of the probe, light source, and sample on a plasticised cloth mat. This was fairly effective, and by positioning the samples correctly the effects of the mat were minimized. To reduce or remove unintentional mixing from the mat, a black cloth was used as a backdrop behind the sample. However, in some cases there was difficulty making the sample lie flat. For reference, the mat's spectrum is shown in Figure 16 below:

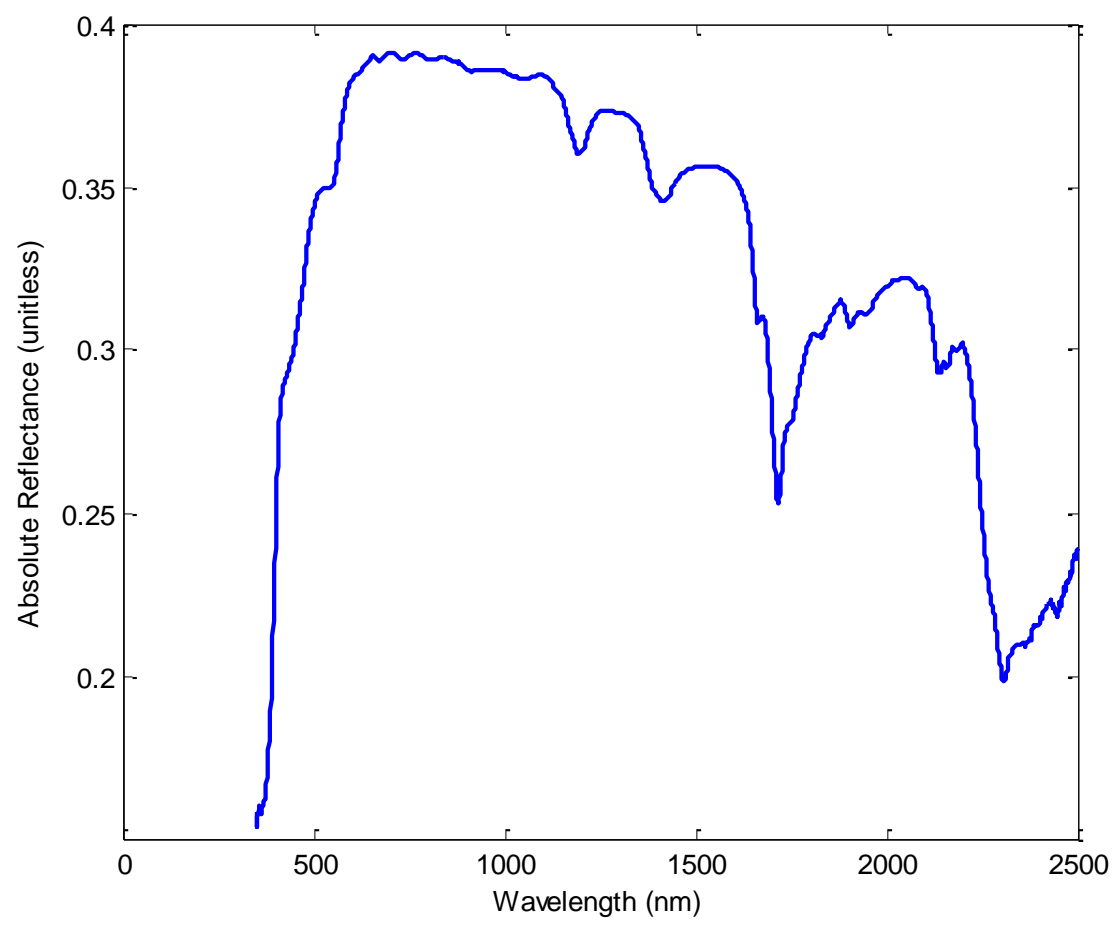

Figure 16. Wet Erase mat used as location marking tool.

A black cloth behind the sample mitigated mixing of the mat's spctra with the sample spectra 
Figure 17 shows the results of the outgassing test on a sample of Kapton HN in the glass bell jar vacuum chamber. This preliminary measurement was a main determining factor in the decision to take spectral measurements in the spectrometry lab, rather than attempting to black out the bell jar vacuum chamber. Specifically, by comparing spectral measurements of kapton (and subsequently the other materials) between the evacuated bell jar and the dark room facility (not evacuated) it was shown that outgassing effects can be successfully measured without being under constant vacuum.

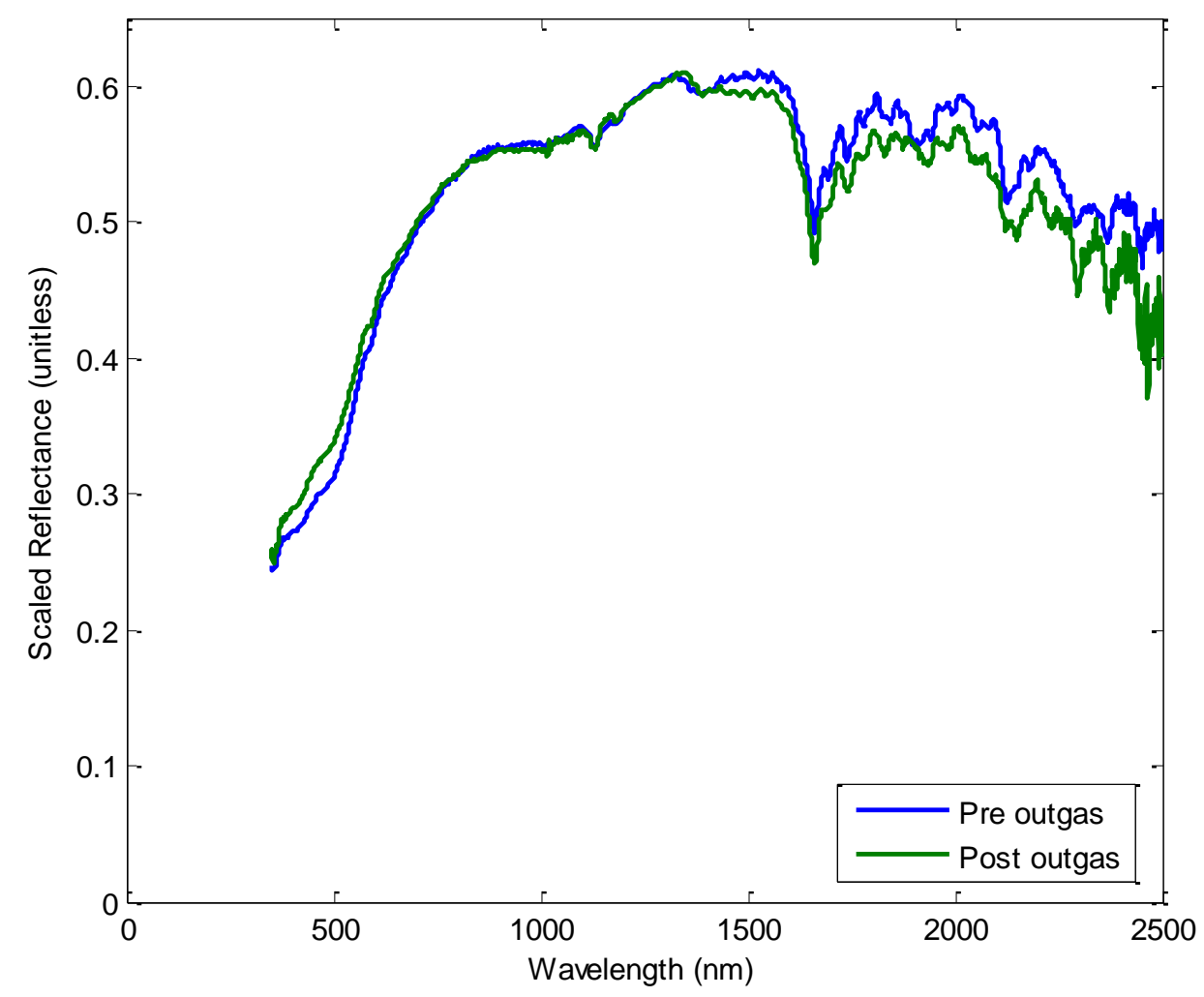

Figure 17. Kapton outgas test results from initial testing in the glass bell jar vacuum chamber.

Shown above are the results of the spectral measurements before and after a kapton film sample. 
Based on the limited availability of the Veeco 747 vacuum chamber, it became necessary to take measurements using a second vacuum chamber. The procedure here was very similar to the procedure for measuring in the Veeco 747 , with the exception of measuring under vacuum, due to the construction of the chamber. The second vacuum chamber has aluminum walls, with a plexiglass cap. The height of the chamber made taking measurements under vacuum infeasible. However, the first tests, as is shown in this section, showed that the outgassing effects were not diminished by removal from the vacuum environment, at least long enough to make measurement in the dark room environment practical. An strong attempt was made to take measurements within five minutes of sample removal from the vacuum environment.

Outgas testing was performed on 11 samples, many of which were a reflective coating overlaid on a kapton or other film blanket, typically used in thermal control. The total list of materials is presented below in Table 2:

Table 2. Materials on which outgassing effects were measured.

\begin{tabular}{ll}
\hline Material & Material \\
\hline Teflon x Silvered Inconel & Aluminum x 1mm 100XC Kapton \\
Germanium x 100CB Black Kapton & 2 mm Kapton x Aluminum \\
2mil Kapton x Aluminum & Aluminum Beta Cloth \\
Solar Cell TRMM-PLBBSA0510 & Uncoated Aluminum PolySat Frame \\
Kapton Tape & PolySat Coated Black Circuit Board \\
ITO x Kapton & \\
\hline
\end{tabular}

Before measurements were taken, it is worth stating that there are a pair of features that are sometimes present in the pre-outgas measurements, but 
should be removed partially to completely in the post-outgas measurements. The two absorption features, at $\lambda=1450 \mathrm{~nm}$ and $\lambda=1950 \mathrm{~nm}$, are representative of water absorbed into the material. These features will not be present in a large number of spacecraft materials that do not absorb water significantly. Another important note is all the following spectra have been normalized to the same relative magnitude to provide an accurate comparison between the two spectra, as most magnitude differences here are due to orientation error between pre and post outgas. The spectra presented in this section were normalized with a function written to normalize one spectrum to another automatically selecting a flat region of the target spectrum to normalize against. The normalization point is noted in the figure caption.

The first material presented is Aluminum Beta cloth, a glass filament cloth with an aluminum coating for thermal control. A reference photograph is shown in Figure 18.

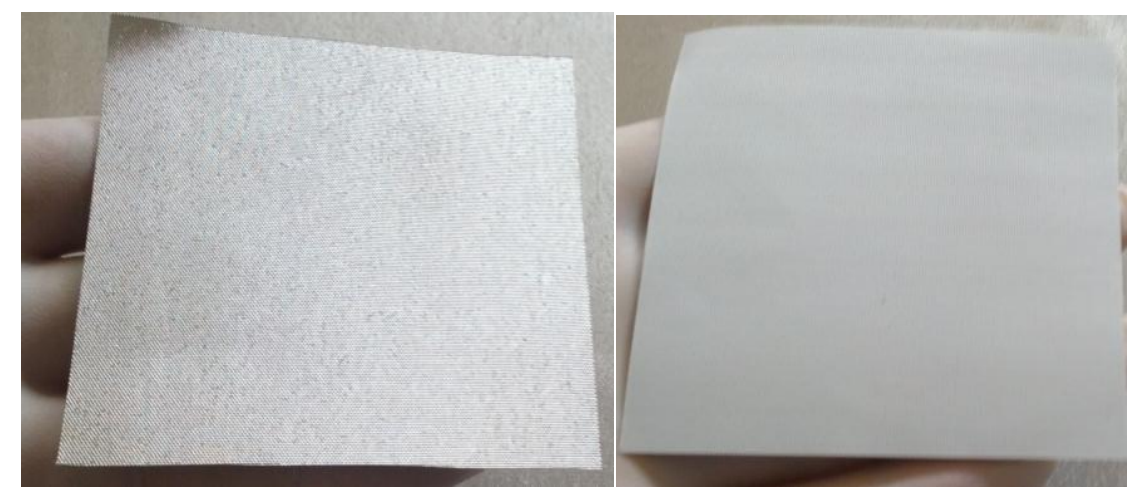

Figure 18. Aluminzed Beta Cloth reference photos.

Shown above are reference photos for both sides of Aluminized Beta Cloth, the aluminized side on the left, with the cloth side on the right. 
As shown in Figure 19 and Figure 20, neither side of the material demonstrates a loss or gain of features.

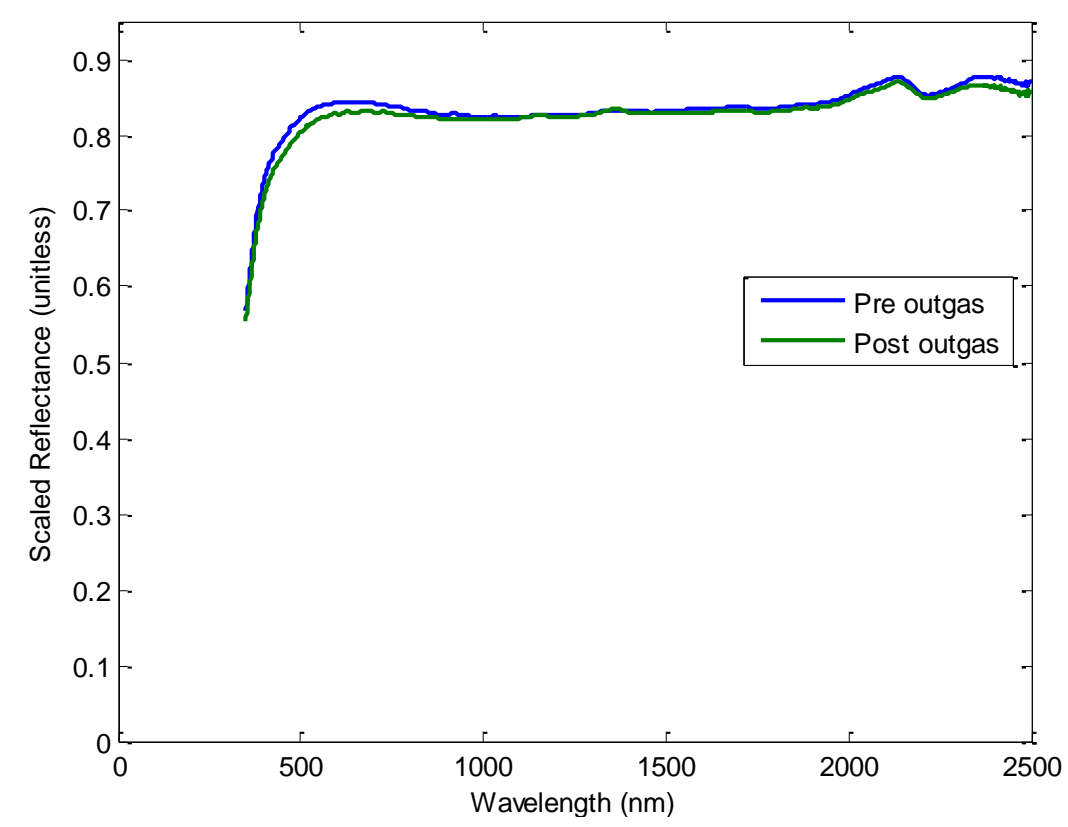

Figure 19. Aluminum Beta Cloth outgas measurements, Beta Cloth side. Normalized to $1100 \mathrm{~nm}$.

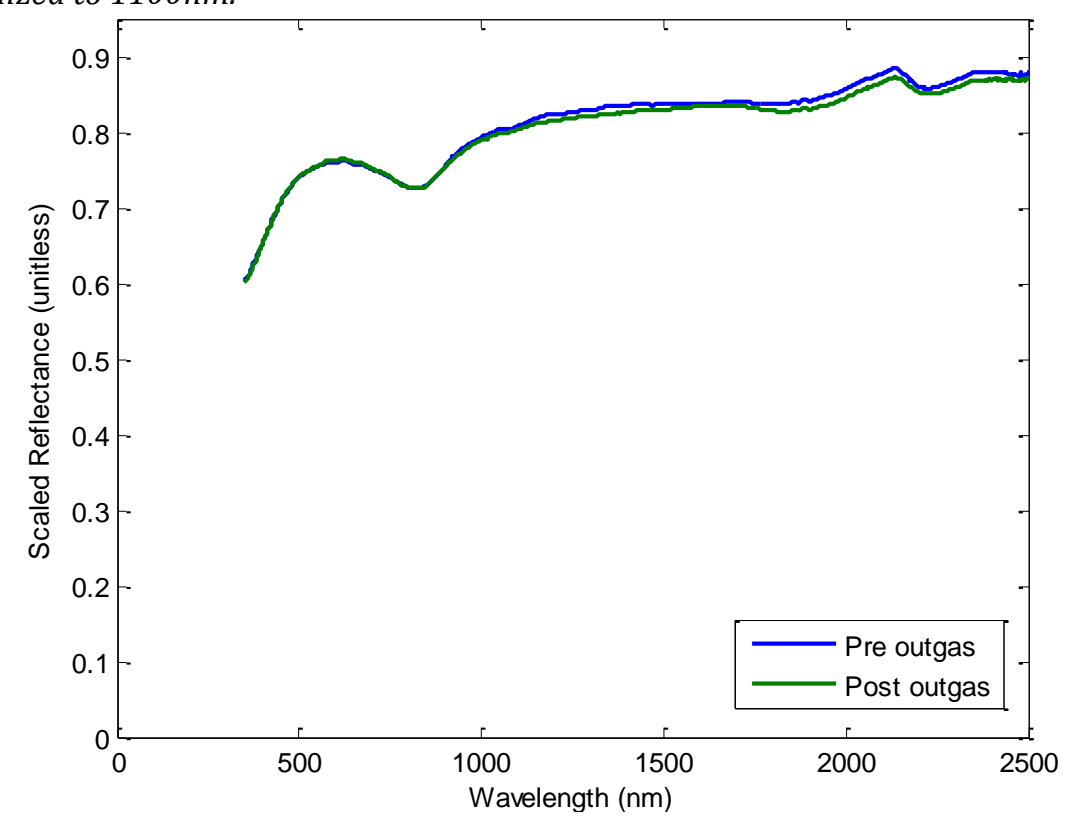

Figure 20. Aluminum Beta Cloth outgas measurements, Aluminum side. Normalized to $920 \mathrm{~nm}$. 
When there is not an appreciable difference between the pre and post outgas spectrum the material is in a category of materials that are only affected by more permanent appearance changes, and can be measured successfully outside of the vacuum environment at any point post-test.

ITO x Kapton is a thermal blanket material, ITO reflects strongly in the infrared and is used for thermal control. This high reflectivity prevented the measurement of the ITO side of this material, but considering the makeup of the material, it is unlikely that it experiences significant outgassing effects. Shown in Figure 21, measurements of the kapton side of the film have the appearance of the ITO spectrum, with Kapton absorption features.

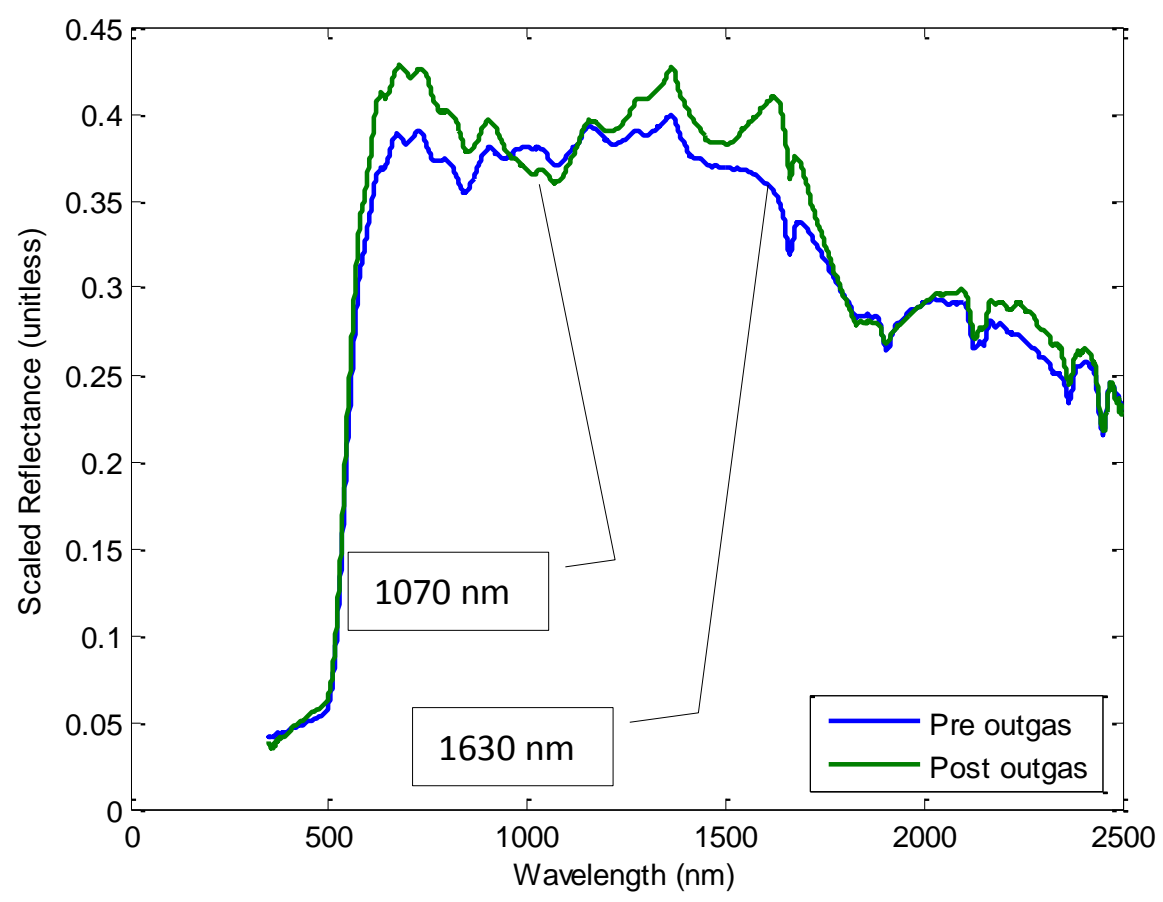

Figure 21. ITO x Kapton, Kapton side. Normalized to $1630 \mathrm{~nm}$. 
There are two major differences between the pre and post-outgas measurements. The appearance of a double absorption feature at $1010 \mathrm{~nm}$ and $1070 \mathrm{~nm}$, and the disappearance of an absorption feature / appearance of a reflectance feature near $2140 \mathrm{~nm}$. The double feature near $1000 \mathrm{~nm}$ was observed in the bell jar effects test, though the feature near $2040 \mathrm{~nm}$ was not. The fact that the absorption feature is not present in the pre-outgas measurements indicates that it is not a water feature, and it bears no resemblance to the features of any of the background material. The source of these features is unknown, however there are some piece of information about the source that can be gleaned. The expected mode of feature generation in outgassing is an existing absorption feature disappearing as the material responsible for that feature leaves the sample. This is not the case in these two features, which leads to the conclusion that whatever is happening is a surface layer change. Perhaps a thin layer of material leaves the surface when exposed to vacuum, and exposes the underlaying layer, which gives rise to the two features seen above.

Germanium x Black Kapton film is used on spacecraft for charge dissipation. Reference photos are shown in Figure 22, and the measurements are shown in Figure 23 below. 


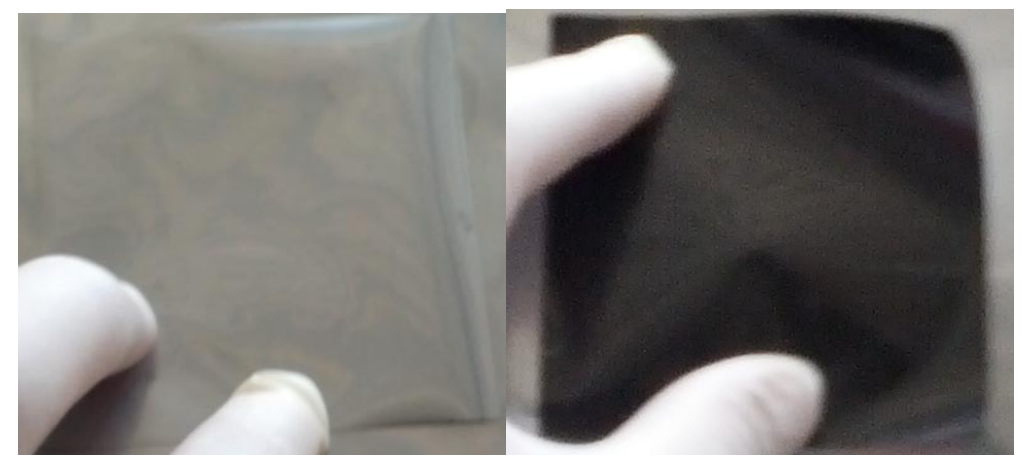

Figure 22. Reference photo for Germanium x Black Kapton.

On the left is the germanium side, used for electrostatic discharge, on the right is the black kapton film side.

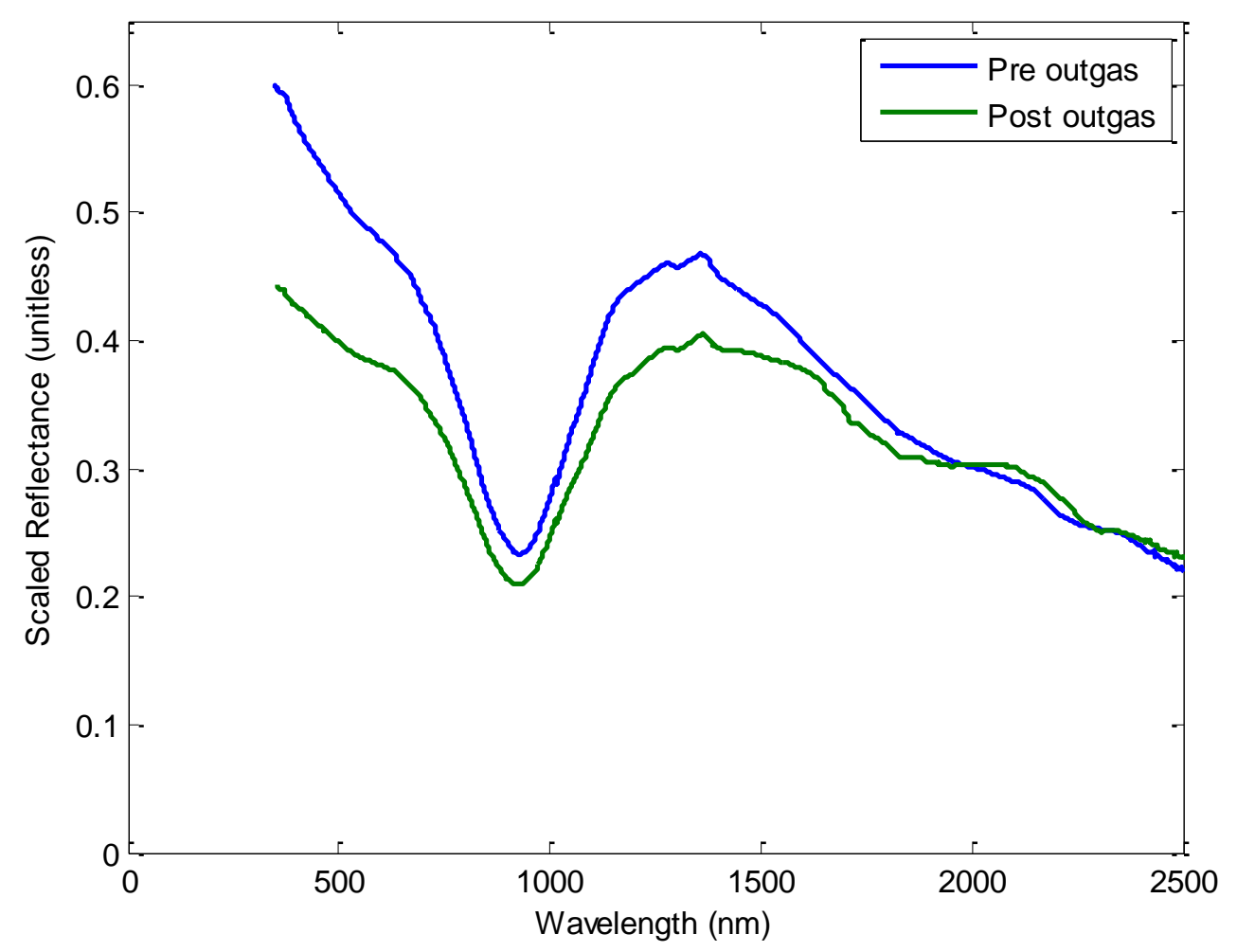

Figure 23. Germanium x Black Kapton Film outgas, Germanium side. Normalized to $2300 \mathrm{~nm}$. 
There is a small change between the pre and post outgas spectra, the magnitude difference is most likely due to change in orientation, and was not fully corrected for by the normalization. There is a shallow absorption feature between $1550 \mathrm{~nm}$ and $2070 \mathrm{~nm}$ on the post-outgas spectrum which does not appear in the pre-outgas spectrum. It is unlikely to be a foreshortened feature, as the other shallow absorption feature near $2250 \mathrm{~nm}$ (which is slightly shifted between nominal and post-outgas) is still present. However, the feature is most likely anomalous, as it only shows up in one of the three spectra combined to create this comparison.

An interesting phenomena to note, is that the material curls significantly after exposure to the vacuum. Given the static discharge usage of germanium, it is likely the chamber venting process imbues a small static charge to the germanium side of the film, causing it to curl. This, coupled with the dark appearance of the black kapton made acquiring a good reflectance spectrum difficult, and an outgassing comparison was not made for the black kapton side.

Teflon x Silver Inconel shows a similarly small change in the material properties between pre and post outgas. Shown below in Figure 24 is a pair of reference photos for Teflon $\mathrm{x}$ Silver Inconel, and Figure 25 shows pre and post outgas spectra for the Teflon side of the Teflon x Silver Inconel. 


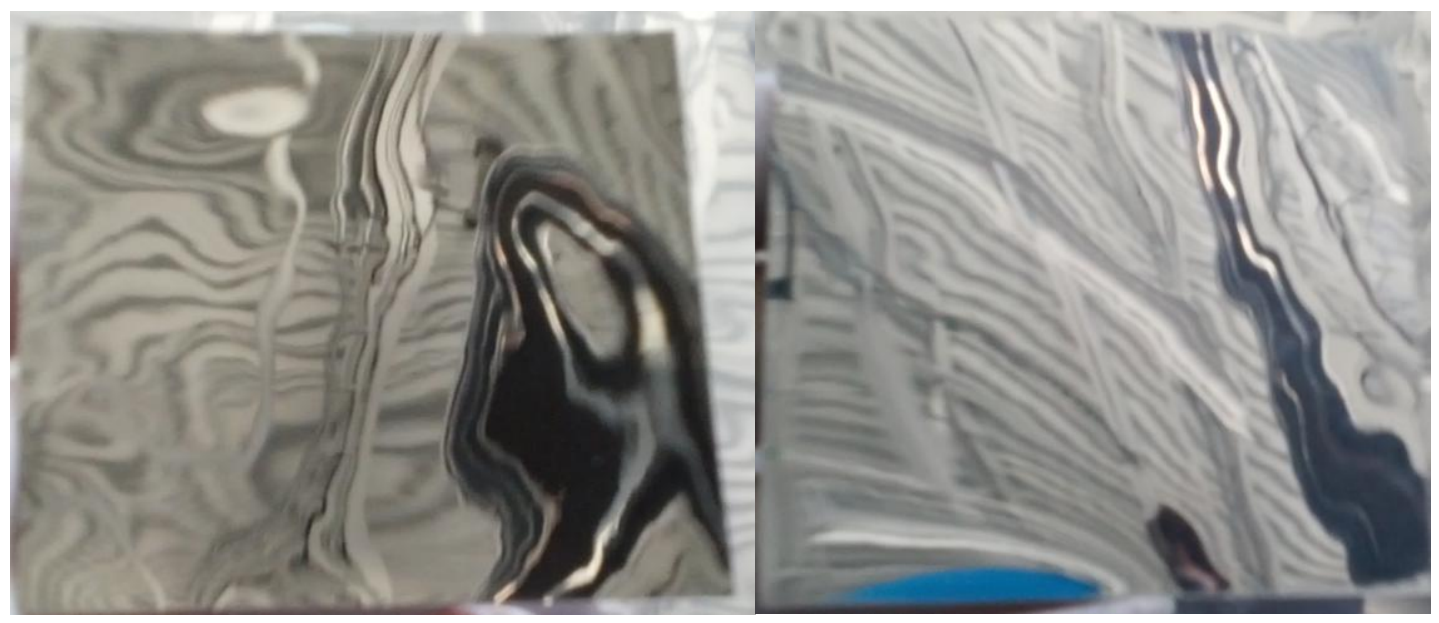

Figure 24. Teflon $x$ Silver Inconel reference photos.

On the left is the exposed silver inconel side, on the right is the teflon film, which is semitransparent.

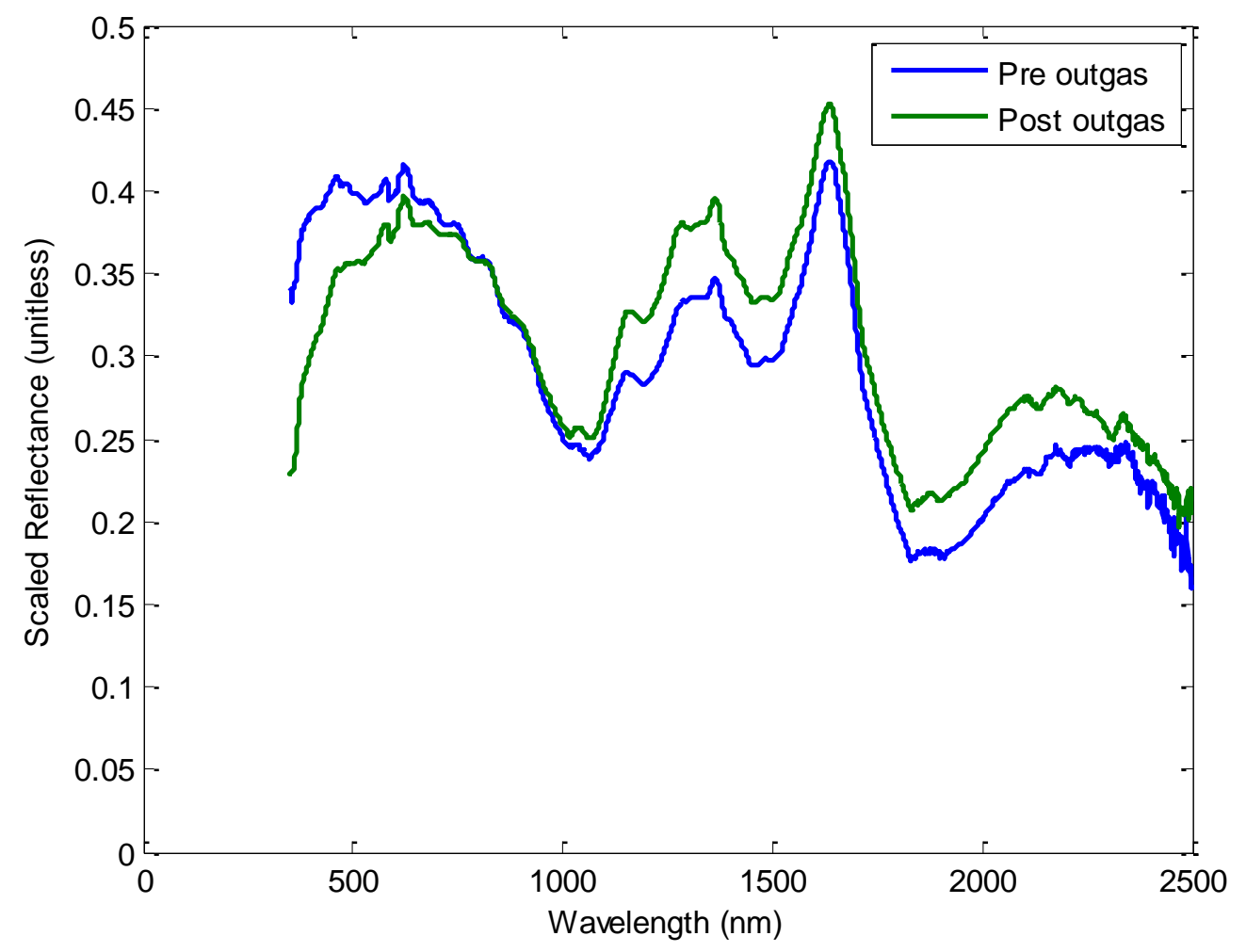

Figure 25. Teflon $x$ Silver Inconel, Teflon side.

Normalized to 800 $\mathrm{nm}$. 
The major difference between pre and post outgas of the Teflon side is a reflectance feature at $460 \mathrm{~nm}$, present in the pre-outgas spectrum, but absent post. The reverse side (Silver Inconel) was too specular to acquire non-saturated measurements on. However, based on the material composition, it is not expected to have significant feature gain or loss due to outgassing, though the Teflon side should exhibit some darkening when eventually exposed to AO and VUV [24]. Similar to the Kapton above, it is likely that the feature gain here in the visible region (at $460 \mathrm{~nm}$ ) is a surface change exposing the lower layer of the material. It is also possible that this feature gain is anomalous, as it only shows up in two of the four post-outgas spectra for this material.

The last film material presented here is aluminum $\mathrm{x}$ Kapton, of which there were several variations, all of which the aluminum coated side showed no effects due to outgassing. The reference photos for Aluminum x Kapton are shown in Figure 26. However, the Kapton showed some feature change, shown in Figure 27. 


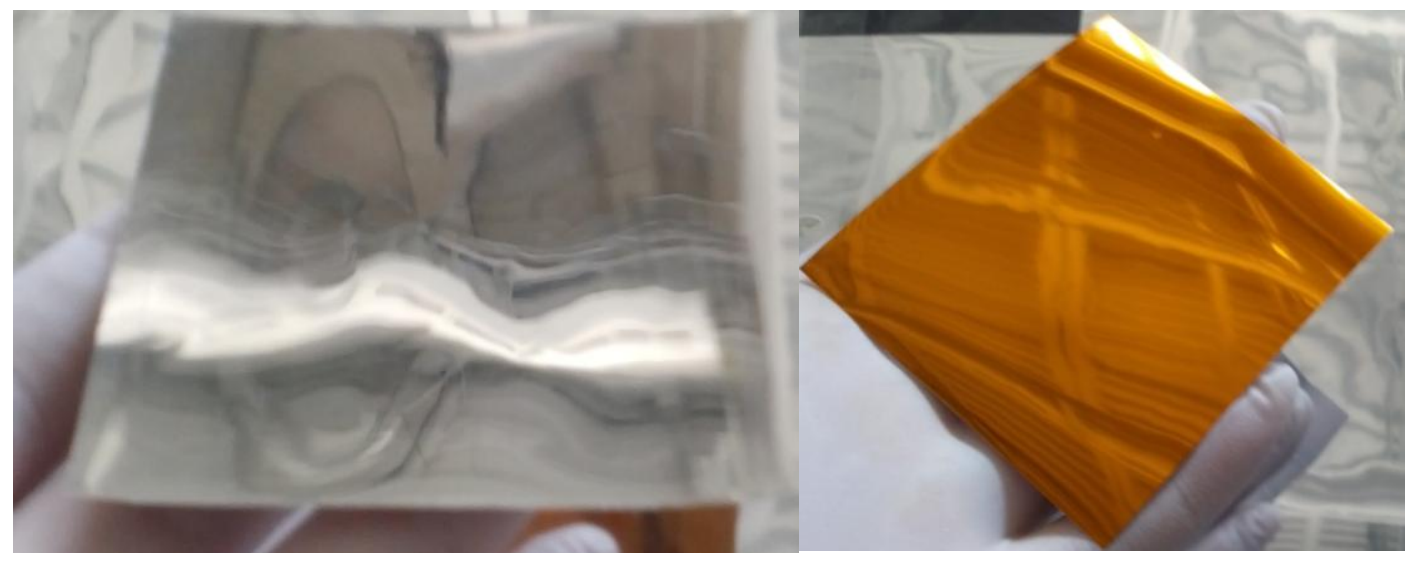

Figure 26. Reference Photos for Aluminum x 2mil Kapton

Shown on the left is the reflective aluminum coated side, on the right is the kapton film side.

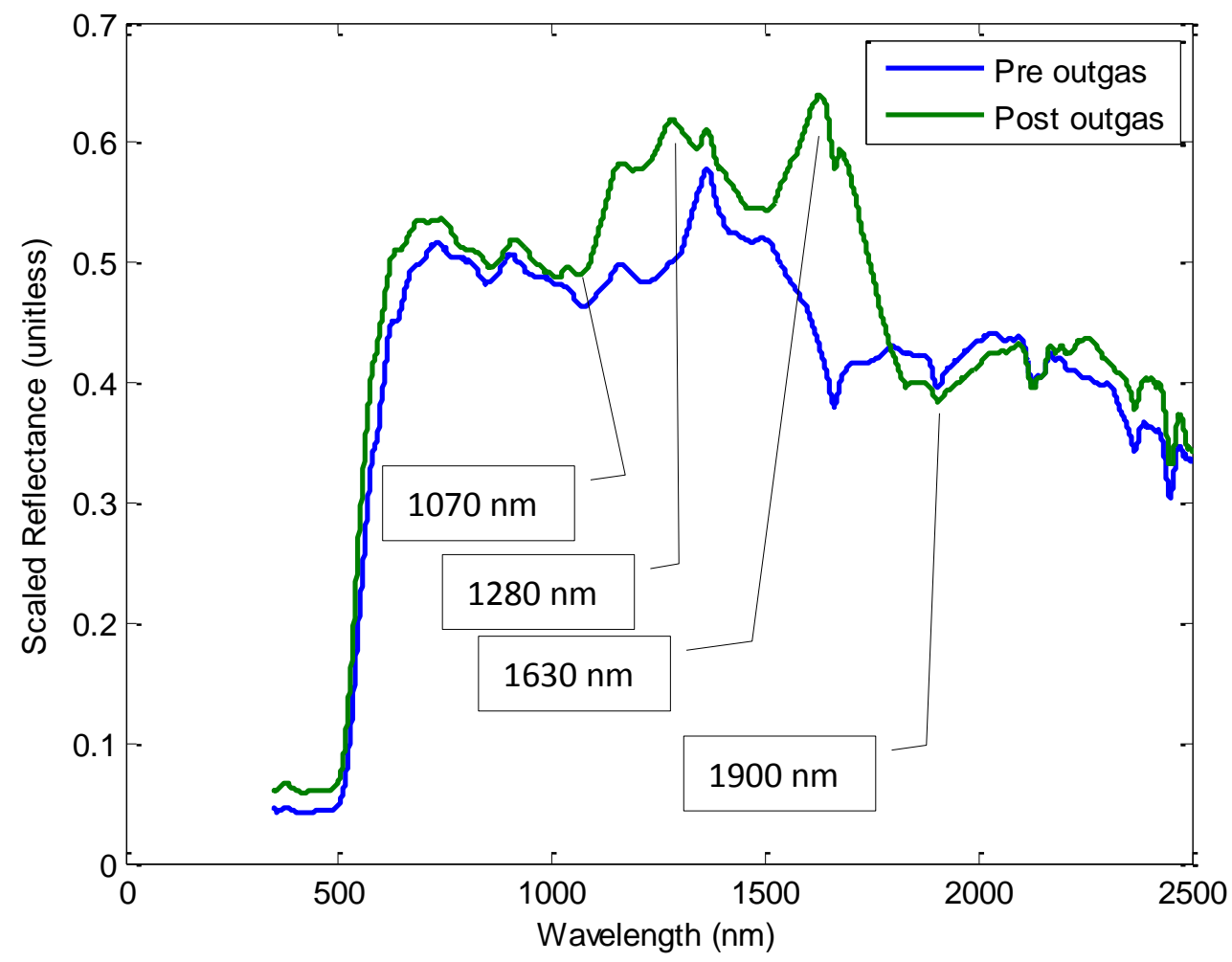

Figure 27. Aluminum x Kapton, Kapton side.

Normalized to $2130 \mathrm{~nm}$. 
Note that there are two main features which are different between the two measurements, an absorption feature lost at $1630 \mathrm{~nm}$, and a reflectance feature gained with a peak at $1280 \mathrm{~nm}$. There is also what appears to be a weaker double absorption feature gained at $1010 \mathrm{~nm}$ and $1070 \mathrm{~nm}$, as on other forms of kapton. The last feature change which is due to outgassing is the feature near $1900 \mathrm{~nm}$, typically due to water. This feature is still present in the post-outgassing spectrum, indicating that there is still water bound up in the kapton, possibly due to the manufacturing process.

The last materials outgassed were a set of cubesat materials acquired from the Cal Poly PolySat team. As per normal, there was little difference in features between pre and post outgas on the uncoated aluminum frame. Changes were observed in the spectrum for the black circuit board. This is expected, due to the organic and often semi-porous nature of the coatings used. Figure 29 shows the pre and post outgas measurements and highlights the differences. Again, the $1900 \mathrm{~nm}$ feature is typically associated with water, and the low magnitude of the absorption feature and absence of the feature at $1400 \mathrm{~nm}$ is indicative of the low water content in the material. The two features at $1095 \mathrm{~nm}$ and $1320 \mathrm{~nm}$ are unknown in origin.

The solar cells used in this test are inherently difficult to measure the outgassing effects on, due to the sinusoidal feature in the infrared and far 


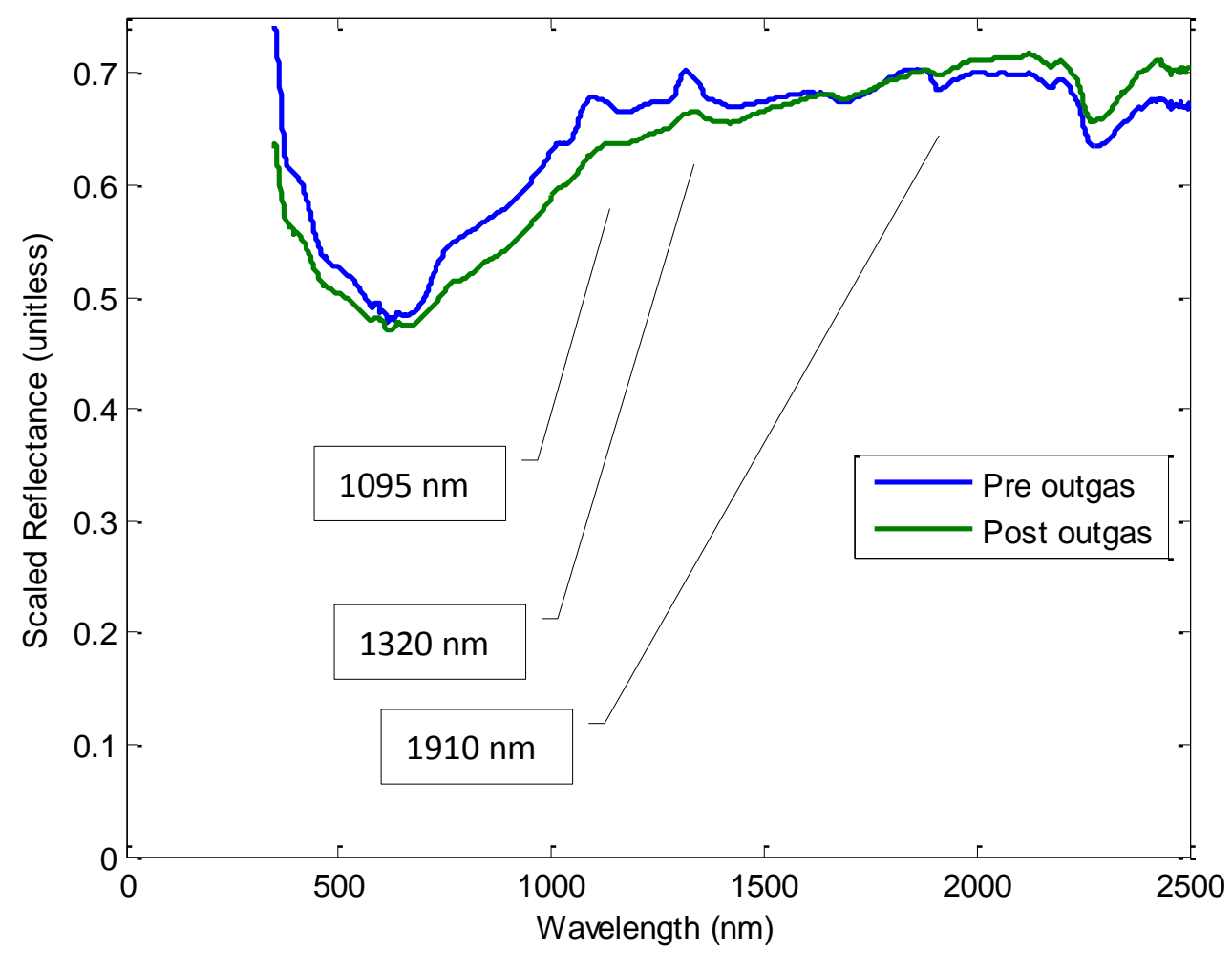

Figure 28. PolySat Black Circuit Board outgas test. Normalized to $1660 \mathrm{~nm}$.

infrared regions. However, no changes were measured in numerous experiments with the solar cells, and this is corroborated by the crystalline nature of the solar cells, considering the cells are not porous, and have a thin film as an unintentional protective barrier to absorption of gasses into the material.

Overall, several conclusions can be drawn from the outgas test. First, most materials measured do not experience any measurable outgassing effects on their spectra. There are several effects on kapton and teflon which are likely a result of surface characteristic changes, indicated by two markers; first the 
Chapter 2 - Spectral Measurements | $\mathbf{5 3}$

appearance of 'reflectance' features, and second, the AO erosion eliminating some of those features on Kapton, as seen in the following section. Overall, outgassing has a measurable effect on several of the materials presented here, and with some exceptions is fairly predictable.

\section{3 - Atomic Oxygen Procedure and Measurements}

For the Atomic Oxygen effects, measurement in the original vacuum chamber becomes more infeasible without modifying the method of supporting the samples within the chamber. Due to the apparatus for producing atomic oxygen in the chamber, it was necessary to remove the samples from the chamber to take measurements, and due to time and material constraints, only the effects on Kapton were measured. Figure 30 shows the results of the atomic oxygen erosion.

It is important to note that both of these spectra are outgassed. There are several important features in the comparison. First, the double feature at $1010 \mathrm{~nm}$ and $1070 \mathrm{~nm}$ is present in the uneroded sample, but not in the eroded. This would seem to indicate the source of the double absorption feature is a surface characteristic, eroded by the atomic oxygen, but gained in the outgassing (as seen earlier, this is not present in pre-outgas kapton samples). There are several other shallower absorption features present in the uneroded sample, but 
Chapter 2 - Spectral Measurements | 54

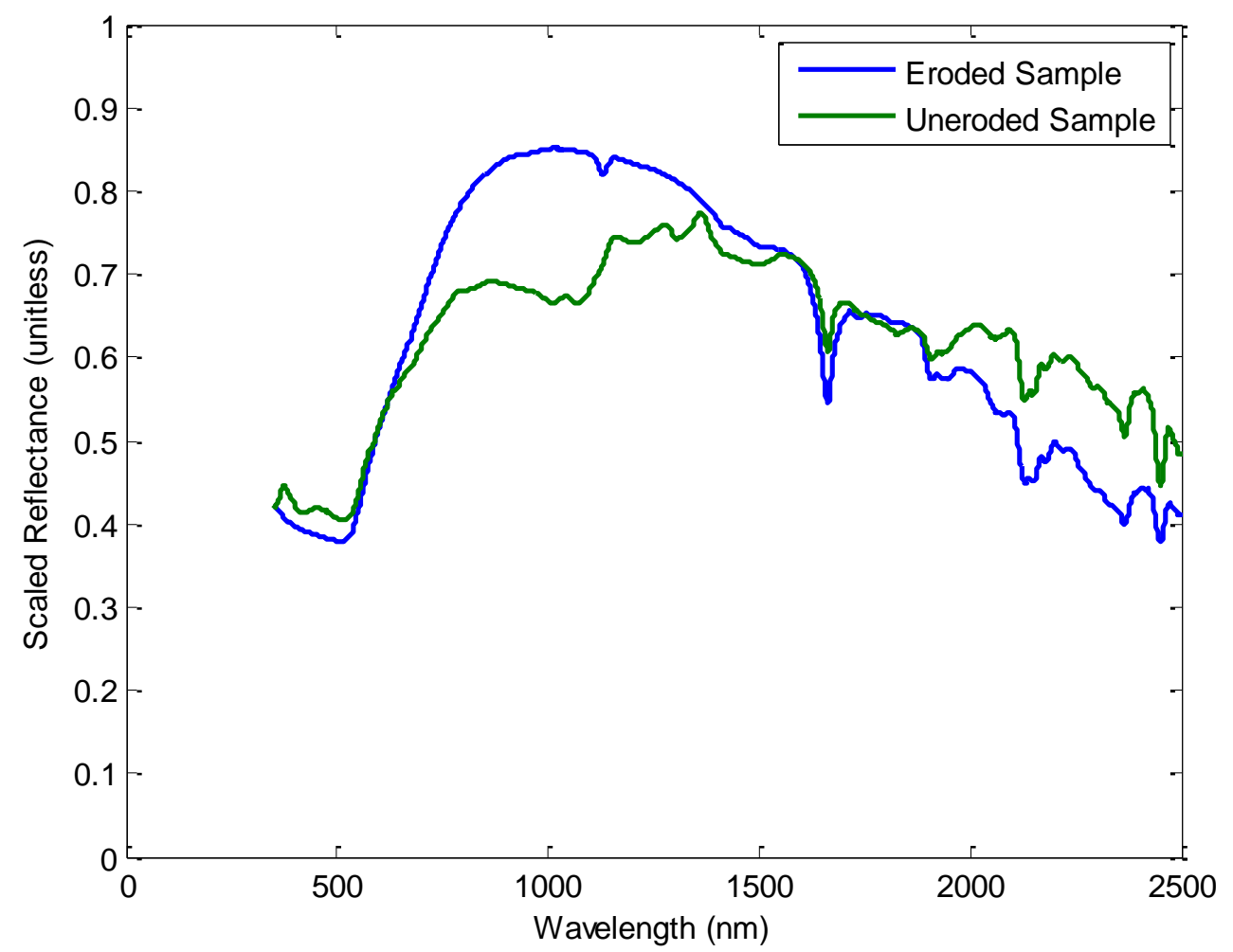

Figure 29. Atomic Oxygen Erosion effects on Kapton HN control experiment. Normalized to $1580 \mathrm{~nm}$.

not present in the eroded up to about $1550 \mathrm{~nm}$. These features are, however, present in non-outgassed samples, and are likely due to a surface characteristic rather than gained through outgassing. It is unlikely that the shallow absorption features are due to orientation differences (though, there is a slope difference between the general trends of the two samples, which is possibly due to orientation difference). To remove uncertainty on the feature genesis it would be recommended to polish the uneroded sample, to remove surface contaminants (and is likely to be important for future outgas tests as well). Past $1500 \mathrm{~nm}$, the features are fairly consistent between the two spectra, with a depth difference between the $1900 \mathrm{~nm}$ water feature on the two, which would seem to 
indicate the water is tied up in the material, and is more easily outgassed when the surface is eroded, though not significantly.

It is also informative to view the absolute reflectance spectra for the atomic oxygen experiment. Since the measurement on the eroded kapton and the uneroded kapton could be made in the same setup, without orientation changes, the absolute reflectance comparison can provide information on the relative magnitude of the reflectivity, as well as shape. This is shown in Figure 30 below:

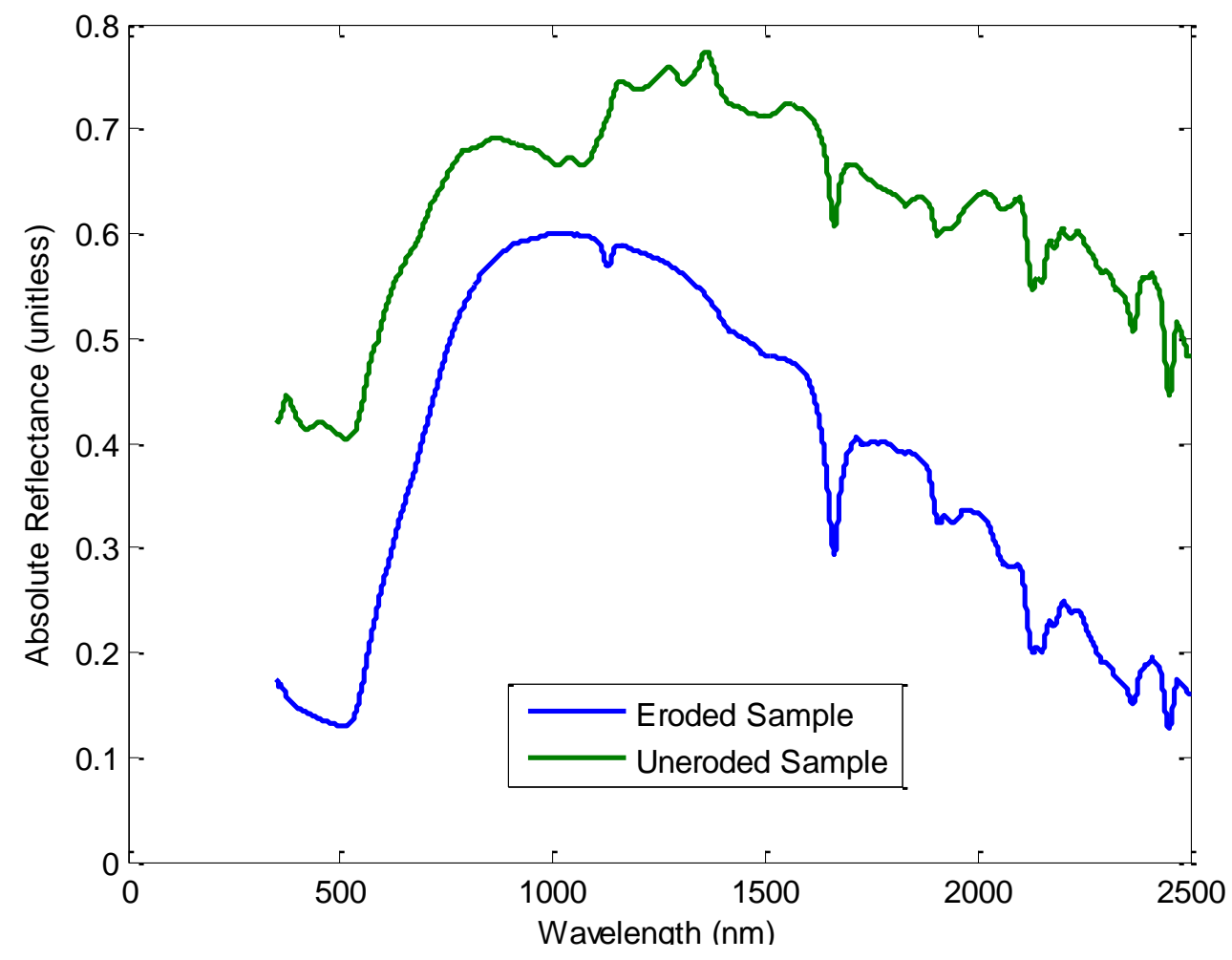

Figure 30. Absolute Reflectance comparison for Atomic Oxygen Erosion of Kapton HN. 
Chapter 2 - Spectral Measurements | 56

Figure 30 shows the change in magnitude between the eroded and uneroded samples. This was apparent by visual inspection as well: the eroded sample appeared to be somewhat duller than the kapton. This is likely due to the 'roughening' of the surface by the atomic oxygen.

Unfortunately, the Kapton HN was the only material tested in the AO apparatus due to time constraints. There are some interesting effects expected when observing both the atomic oxygen erosion, the vacuum ultraviolet degradation, and the synergistic effects between the two, once the apparatus is available for testing. The atomic oxygen erosion measurements are important for an accurate modelling of space environment effects as the basis of the unmixing model when identifying space objects, and should be relatively easily incorporated into the model once acquired.

\section{4 - Improvements}

In the course of making spectral measurements of outgas, A0, and non space-environment and reviewing the measurements, there are several changes to the test procedure that would improve the quality of the measurements. First, creating a better system of maintaining consistent orientation between the original measurement and the post space weathering measurements take place. Another improvement would be to ensure the samples are as uncontaminated as possible, cleaning the surface before exposure to the vacuum chamber. A timed 
Chapter 2 - Spectral Measurements | $\mathbf{5 7}$

spectral measurement test was attempted with the expectation of interesting results. However, the material choice was poor, as none of the materials chosen exhibited outgas removed features. The test procedure involved simply removing a sample from the vacuum chamber and measuring its spectrum every few minutes, then every few hours (as over the course of a few minutes the spectrum remains quite constant, with no appreciable change in the spectrum). The time increment may have to increase to days, depending on the timeframe for 'regassing'. Good choices of materials for this test would be kapton film, any component with an organic coating (such as epoxy, or organic paints), or any material that exhibits outgassing effects.

Analyzing the atomic oxygen measurements revealed an easy method for removing the problem of varied orientation between the pre- and post-outgas spectral measurements. All that is required is a second sample, which is not outgassed, but is prepared in the same way (cleaned / kept clean). No preoutgas measurement is necessary with this method, simply take the two samples, measure the outgassed sample first, then maintaining the same setup, measure the second, non-outgassed sample. This maintains nearly identical relative orientations for the two measurements, and eliminates some setup time.

A major set of measurements that would greatly improve the understanding of how the measurement conditions change the spectrum would 
be an orientation-specific measurement. Capturing the spectrum of a material from a grid of orientations relative to the incident light beam. The incident beam should not need to be moved during this test, as moving it should change only the orientation of the principle reflection axis. A setup capable of performing this measurement would also be ideal for taking measurements before and after space weathering testing as it would need to be capable of recording the angular position (likely as sum of vectors).

\section{5 - Conclusions}

There are a few conclusions that can be made from these measurements. First, regarding the measurements made on spacecraft materials, it would certainly be possible to group these materials into categories for unmixing, then selecting the best possible individual material out of the category. The method has some problems, as it ignores the possibility of multiple individuals from one category on a single surface. Second, regarding the outgas measurements, the purpose of the testing was twofold: to measure the outgassing response of some spacecraft materials to serve as a basis for application of the unmixing model to spacecraft or objects on orbit, and as an exploratory experiment, to determine the best methods for measuring materials after being exposed to a vacuum. Both of these were accomplished, though a much larger set of materials will need to be measured to serve as a proper basis for unmixing on space objects. Last, the atomic oxygen experiment was only a precursor to further testing. However, the 
Chapter 2 - Spectral Measurements | 59

measurements did seem to indicate the strong spectral effects (at least on some materials) of atomic oxygen erosion, which will warrant further experimentation to provide yet another set of bases for unmixing.

The end goal of these experiments will be to form a full basis of material spectra with various space environment effects, a set with just outgassing, a set with plasma arcing scars, etc. for each material. This basis will then be used for identification, to identify not only the materials on the object, but the condition of the materials as well. 


\section{3 - Spectral Unmixing}

Spectral unmixing is the process of inverting material proportions from a combined spectrum that has distinct components that are linearly mixed. To solve the problem, two main methods were explored to deal with the large solution space. The second major method explored turned out to be significantly more efficient, though equivalent in the estimated accuracy it could achieve.

\section{1 - Genetic Algorithm}

Starting from previous manual methods of determining material proportions, the first unmixer constructed was a simple Monte Carlo guesser. This used a large $(\sim 10,000)$ set of possible spectral combinations randomly generated normed to a total of 1 for material proportion. This initial assumption that the material proportion should be restricted to sum to $1(100 \%)$ turned out to be mistaken. Due to the inclusion of BRDF, this material total is often less than $100 \%$, not even taking into consideration the varying BRDF for the angles of the individual material observations. For the purposes of this report and analysis, measurements are assumed to have taken place at roughly the same orientation and possess equal values for BRDF. Expanded methods to improve accuracy should take BRDF into account, though more measurements will be required to incorporate BRDF into the unmixing process. 


\section{Chapter 3 - Spectral Unmixing | 61}

Given that the BRDF values vary between measurements, the next iteration from the Monte Carlo guesser added a rescaling mechanism to the sample setup, to put the material combination on the same scale as the input spectrum. This provided better results for the output and was a preliminary step to the genetic algorithm, which was the goal behind building the Monte Carlo guesser. The next step was the development of a genetic algorithm to narrow the results to a tighter search space.

Genetic algorithms are a powerful method for optimizing a solution to very large problems that cannot be solved directly. In its most general form, a genetic algorithm functions by beginning with an initial guess, or approximate solution, then varies and recombines that solution to form a new best guess. It repeats this process until some minimization constraint is met. In the specific application of spectral unmixing this was implemented using the Monte Carlo guesser as the initial step. The seed for the next step was chosen by calculating the difference between each sample and the input spectrum and selecting the 10 samples with the lowest difference. Creating a 'bubble' around each of these solutions to expand provided the next set of possible solutions, and the process was repeated until a sufficient level of accuracy was achieved. These two parameters, the 'scrunch factor' that decides the size of the solution bubble at each step, and the error constraint, could be set at varying levels. Had the better matrix math solution not been found, the next step would have been to make the solution bubble sizing scale appropriately 
with the accuracy of the intermediate solution. Other improvements were investigated, though ultimately abandoned with the improved solution method outlined below.

\section{2 - Constrained Least Squares}

Partway through the development of the genetic algorithm solution, it was recommended by the committee to investigate other mathematical solutions to the numerical problem. To explain the methodology, it is necessary to look at the problem in a slightly different light. To reiterate from Chapter 1 , in equation (6):

$$
S_{\text {combined }}=\sum_{i=1}^{n} p_{i} B_{i} S_{i}+N
$$

note that $S_{\text {combined }}$ and $S_{i}$ can be represented as very long vectors, with reflectance values at each of the measured wavelengths. This allows an expansion into a vector math representation:

$$
\vec{S}_{\text {combined }}=p_{1} B_{1} \overrightarrow{S_{1}}+p_{2} B_{2} \overrightarrow{S_{2}}+\cdots+p_{n} B_{n} \overrightarrow{S_{n}}+\vec{N}
$$

$p_{i}$ and $B_{i}$ are both scalars, making it quite easy to restate this as a matrix multiplication problem with a known solution:

$$
S_{c}=S A
$$




\section{Chapter 3 - Spectral Unmixing $\mid \mathbf{6 3}$}

Unfortunately, the matrix $S$ is not square so it cannot be truly inverted to solve directly for $A$. So an optimization method must be used, minimizing:

$$
S_{c}-S A
$$

Getting the solution for $A$ which makes equation (11) as close to 0 as is possible. This is accomplished with a pseudo-inverse, which applied to this problem is known as a least-squares optimization:

$$
S^{T} S_{c}=S^{T} S A
$$

Multiplying both sides by $S^{T}$ creates a square matrix that is guaranteed to be invertible:

$$
\left(S^{T} S\right)^{-1} S^{T} S_{c}=A
$$

This function minimizes equation (11), and provides a beginning point for the solution to the unmixing problem. Testing this solution, for some combined spectra the unmixer returned negative proportion values, which is physically impossible. To rectify this issue a constrained least squares function was used, MATLAB's built in Isqnonneg function. The function uses a modified Lagrange multiplier method to solve the constrained problem. By reframing this as a vector problem, and recognizing it as a minimization problem, it becomes clear that the Lagrange solution is solving the constrained minimization problem:

$$
f=\left(S_{c}-S A\right) \quad A \geq 0
$$


Chapter 3 - Spectral Unmixing | 64

This is solved for the specific $A \geq 0$ case by the lsqnonneg function. To maintain the constraint using a Lagrange multiplier method, the function first calculates the least squares solution, including negative solutions. It then uses those solutions to create a vector of logicals defining which solutions are negative, and need to be corrected. This vector becomes the lagrange multiplier, and the optimization is performed. This process is repeated until an optimum solution is found.

\section{3 - Validation and Error estimation}

A major consideration in solving problems like this is how to validate the proposed solution to the minimization problem, given that when used in practice, knowledge of the object's composition is the goal of the unmixing, and not known beforehand. However, the unmixing algorithm can be validated on test cases where the composition is known, such as the various CubeSats that were measured prior to their launch. Composition and amount of materials can be estimated from pictures taken of the spacecraft. Barring that, however, composition correctness can also be estimated by the spectra included of materials that ought to be present on that CubeSat, for example: the circuit board material spectra between HRBE and AubieSat are distinct, so inclusion of one in the other would be indicative of a mistake or flaw in the unmixing process. In addition to comparing material origin with the materials predicted by the unmixer, a good preliminary check is varifying the values output by the unmixer. A large majority of them ought to be very low or 
Chapter 3 - Spectral Unmixing | 65

zero, since it is unlikely on a cubesat or other spacecraft that a large fraction of the possible materials are present on one side, or in one measurement. A final, easier method of validation is mathematically simulating a combined spectrum, where the composition of the spectrum is known, then unmixing that spectrum. The last form of validation is measuring the error between the unmixed spectrum and the original spectrum. Measuring the error, and showing that the error is low is not a direct validation, as it only shows that the end result of the unmixing works well without confirming that the material combinations are correct.

To estimate the error in the results when unknown spectra are unmixed, the difference between the original and unmixed spectra is calculated (called the residual). Since a vector approximation method is used to calculate the best unmixing solution, the two-norm is calculated, and used for error:

$$
2 \text { Norm }=\sqrt{S^{T} S}
$$

where $S$ is the column vector that contains the reflectance values of the spectrum. This area is then used to calculate the error based on the difference in area.

$$
E=\frac{\operatorname{Norm}_{u}}{\operatorname{Norm}_{c}}=\frac{\sqrt{S_{u}^{T S}}}{\sqrt{S_{c}^{T} S_{c}}}
$$

This error estimation gives a percentage error, and gives an estimation to the cut-off point of significant figures in the output. [25]. 


\section{4 - Unmixing Results}

Discussing the results of unmixing and validation, it is important to once again note that the results presented here may be skewed slightly by the variance in BRDF values. To gain material proportion from these results an assumption is made that the combined spectra were taken at exactly the same angle as the individual constituent material spectra, giving exactly the same BRDF values in each measurement. Variations between the measured proportions and the calculated unmixing values are most likely due to this phenomenon.

The basis spectra used for the simulated mixing and cubesat test cases are shown in Figure 7-Figure 10 in Chapter 2, and in Appendix A, and were measured on AubieSat and HRBE.

There are a few comments to be made about the material spectra used for unmixing: First, many of the materials appear very similar between the two spacecraft, AubieSat and HRBE, most likely due to there being duplicate materials used, perhaps as a base material, or similar coatings. As such, when the spectra are similar between the two spacecraft, it is possible to have a linear combination of the two (or more) materials to account for variations in the full side observation condition relative to the individual material observation condition. Also, due to the wide variation in solar cell appearances depending on orientation and surface characteristics, several solar cell spectra were included for their properties. 


\section{Chapter 3 - Spectral Unmixing $\mid \mathbf{6 7}$}

The first set of validations performed was on simulated combinations, where a combined spectrum formed from a random vector with a sum of 1 was unmixed, and a comparison between the unmixed and simulated mixing spectra is shown in Figure 31 and Table 3 below.

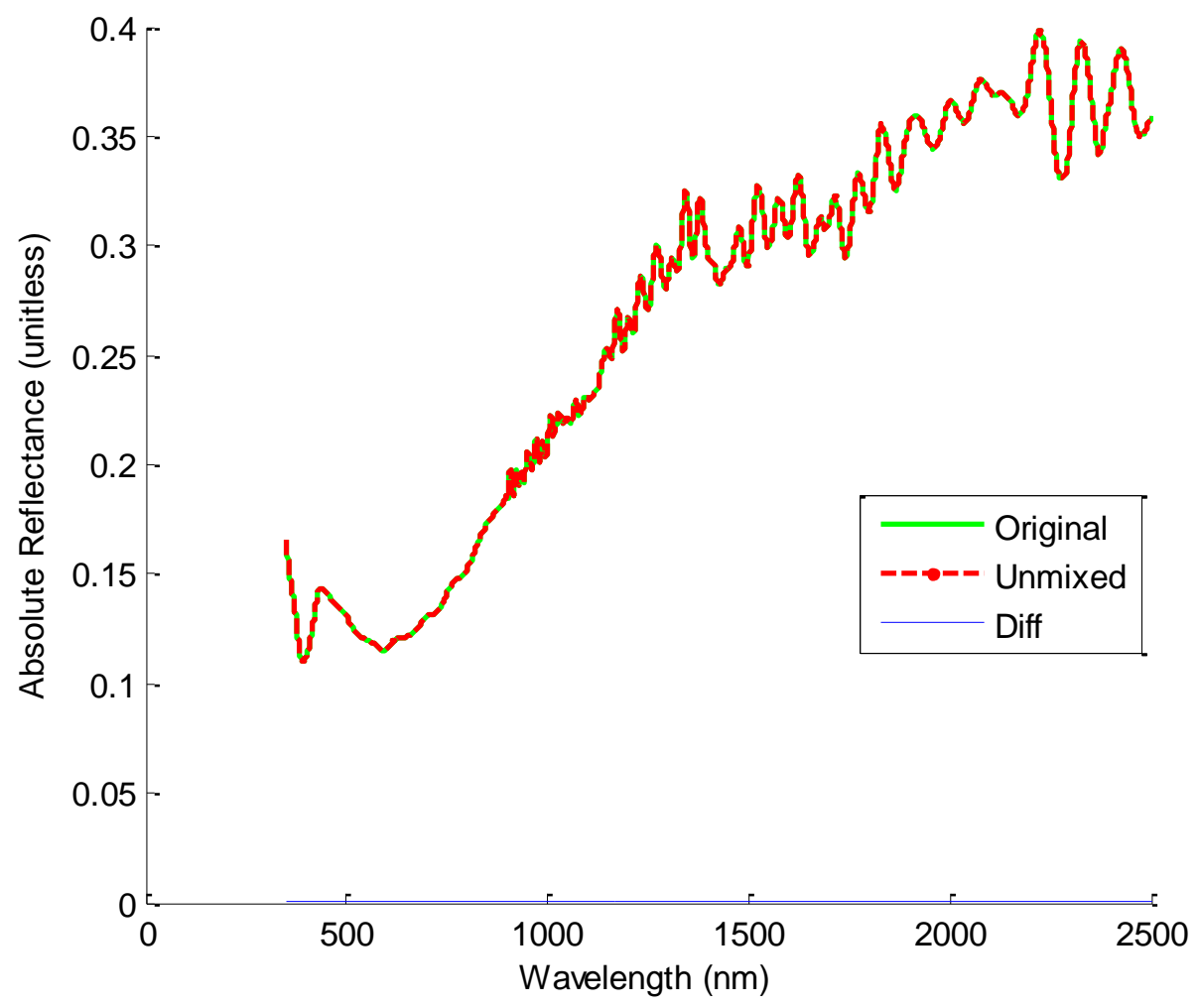

Figure 31. Full vector unmixing results.

Note that the two mixed spectra are exactly on top of each other, and the residual (diff above) is effectively zero across the entire plot. For this reason, the unmixed result is plotted with a dashed line. 
Table 3 Simulated mixing unmixing results with a full vector.

Shown below are the initial material vector values and unmixed values, for comparison. The difference between the two is zero to 6 decimal places, as shown by the vector norm approximation error.

\begin{tabular}{llll}
\hline Sim Vector & $\begin{array}{l}\text { Unmixed } \\
\text { Vector }\end{array}$ & Material \\
\hline 0.037473 & 0.037473 & Solar Cell 1 & (AubieSat) \\
0.006248 & 0.006248 & Solar Cell 2 (AubieSat) \\
0.013144 & 0.013144 & Green Circuit Board (AubieSat) \\
0.111431 & 0.111431 & Uncoated Green Board (AubieSat) \\
0.094025 & 0.094025 & Solar Cell 3 (MSU Sat) \\
0.042910 & 0.042910 & Red Wire with Kapton (MSU Sat) \\
0.128585 & 0.128585 & Red Wire (MSU Sat) \\
0.004661 & 0.004661 & Green Circuit Board (MSU Sat) \\
0.059371 & 0.059371 & White Circuit Board (MSU Sat) \\
0.051633 & 0.051633 & Black Anodized Aluminum (MSU Sat) \\
0.103590 & 0.103590 & Grey Anodized Aluminum (MSU Sat) \\
0.107607 & 0.107607 & Antenna (MSU Sat) \\
0.025288 & 0.025288 & Solar Cell, no sinusoid feature \\
\hline 0.066275 & 0.066275 & Solar Cell, sinusoid feature min TF \\
\hline 0.060297 & 0.060297 & Solar Cell, sinusoid feature \\
\hline 0.087460 & 0.087460 & Solar Cell, sinusoid feature, alt \\
\hline & & \\
\hline
\end{tabular}

The unmixing model also successfully inverts vectors with components missing with the same level of accuracy as no components missing. Shown in Figure 32 and Table 4 is the results from unmixing a random vector with components missing, with the remainder summed to 1 . 


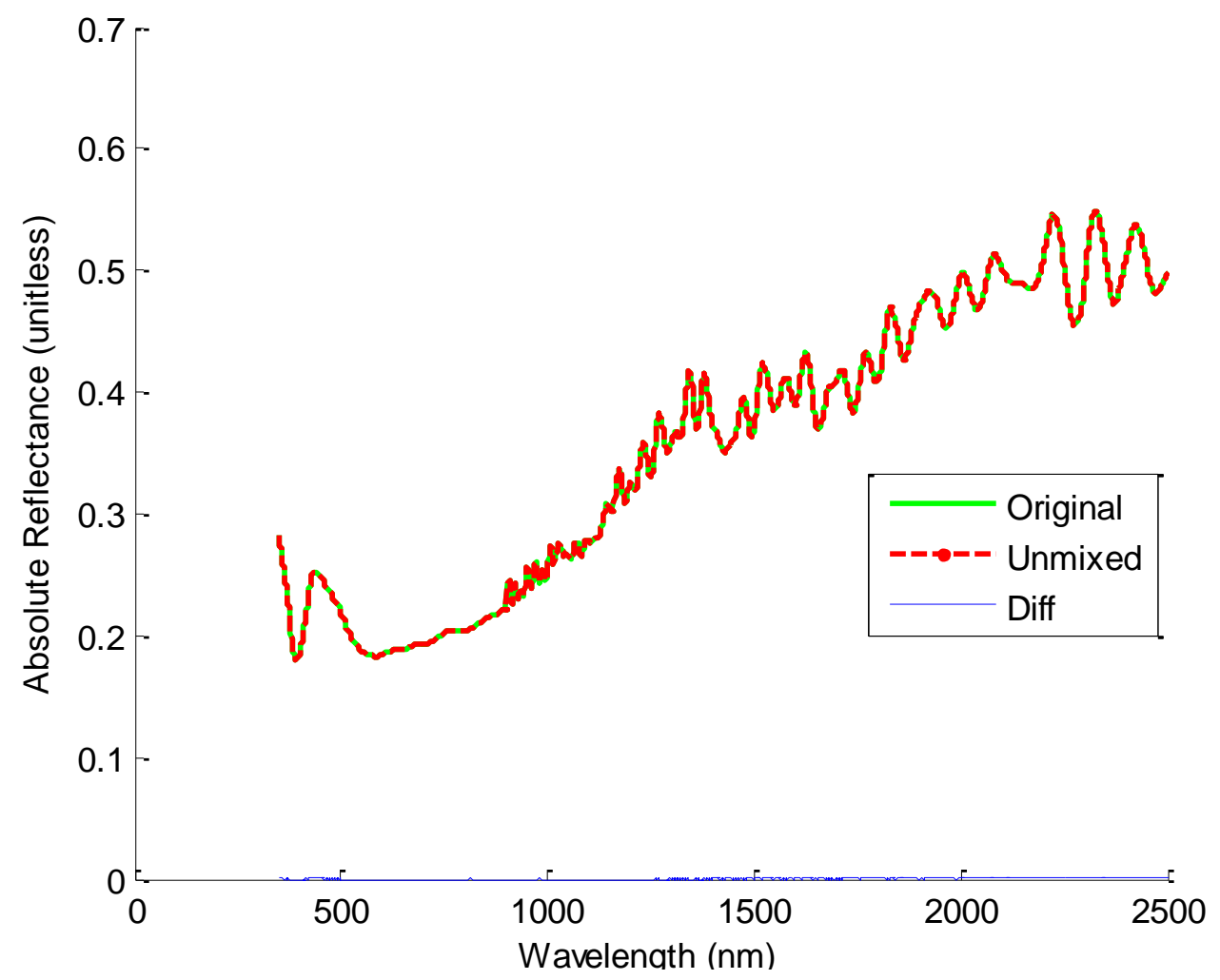

Figure 32. Simulated mixing unmixing results with a partial vector.

Shown above are the results of unmixing a simulated linear mixing of spectra with the full range of possible spectra (no zeros). Note that the original spectrum and the unmixed spectrum are close to perfectly overlapping, which makes the residual (Diff above) effectively zero.

Note again the very low residual (difference in Figure 32), signifying the very low error in calculating a non-noisy, simulated linear mixing inversion. The unmixer also deals with noise quite well. This is due to the fact that the methods used are providing the best approximation to the shape, and the noise doesn't normally significantly change the overall shape, just adds spikes and small variations to the spectrum. 
Table 4. Simulated mixing unmixing results.

Shown below are the initial material vector values and unmixed values, for comparison. The difference between the two is zero to 6 decimal places, as shown by the vector norm approximation error.

\begin{tabular}{llll}
\hline Sim Vector & $\begin{array}{l}\text { Unmixed } \\
\text { Vector }\end{array}$ & Material & \\
\hline 0.000000 & 0.000000 & Solar Cell 1 & (AubieSat) \\
0.000000 & 0.000000 & Solar Cell 2 & (AubieSat) \\
0.000000 & 0.000000 & Green Circuit Board (AubieSat) \\
0.000000 & 0.000000 & Uncoated Green Board (AubieSat) \\
0.159019 & 0.159019 & Solar Cell 3 (MSU Sat) \\
0.000000 & 0.000000 & Red Wire with Kapton (MSU Sat) \\
0.028886 & 0.028886 & Red Wire (MSU Sat) \\
0.000000 & 0.000000 & Green Circuit Board (MSU Sat) \\
0.232969 & 0.232969 & White Circuit Board (MSU Sat) \\
0.082625 & 0.082625 & Black Anodized Aluminum (MSU Sat) \\
0.142068 & 0.142068 & Grey Anodized Aluminum (MSU Sat) \\
0.000000 & 0.000000 & Antenna (MSU Sat) \\
0.000000 & 0.000000 & Solar Cell, no sinusoid feature \\
0.061922 & 0.061922 & Solar Cell, sinusoid feature min TF \\
\hline 0.122816 & 0.122816 & Solar Cell, sinusoid feature \\
\hline 0.169694 & 0.169694 & Solar Cell, sinusoid feature, alt \\
& & \\
\hline & 0.000000 & Vector Norm Approximation Error \\
\hline & &
\end{tabular}

The best case for validation is from a pair of cubesats measured in the Cal Poly PolySat clean room, where the proportion data is available from pictures taken of the cubesats. Unmixing information is available for all sides of the cubesats, and is presented in Appendix B, a selection are presented here for discussion. An important note before presenting the cubesat unmixing results is that many of the materials have a shared spectrum, or very similar features between the two cubesats. Some overlap between materials of the two cubesats is expected. This can 
be seen in the spectra presented in chapter 2. Figure 33 shows a picture of the positive $\mathrm{x}$ side of AubieSat, which is the first unmixing result discussed.

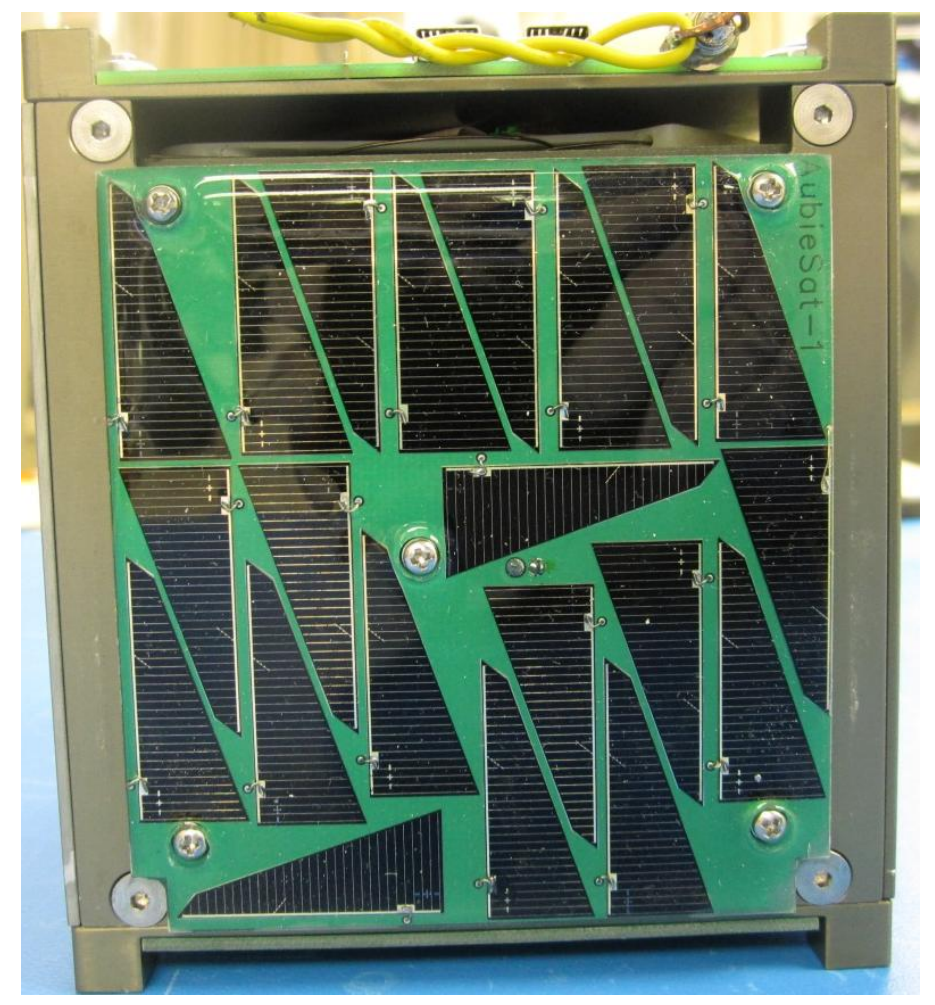

Figure 33. AubieSat Positive $\mathrm{X}$ side reference photo for unmixing.

Shown above is the positive $x$ side of AubieSat. Features to note are the reflection on the surface coating, some kind of epoxy coating which will strongly influence the mixing results in the favor of the spectra with organic features.

Figure 34 and Table 5 show the unmixing results for the positive $\mathrm{x}$ side of AubieSat, with the proportions approximated from the picture for comparison. 


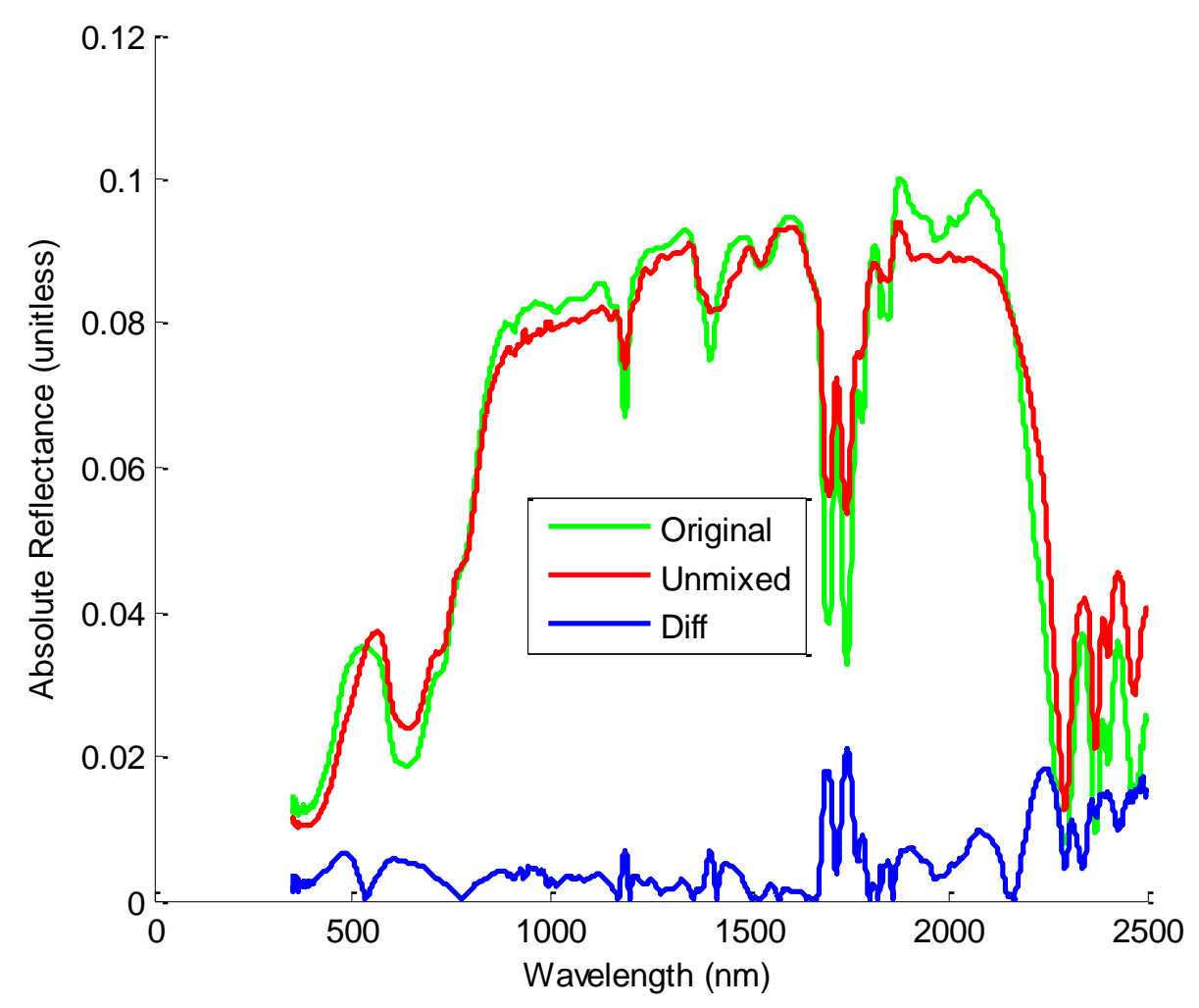

Figure 34. Unmixing results for Positive $\mathrm{X}$ side of AubieSat.

Note the deep organic features and the apparent lack of aluminum features.

Table 5 Unmixing results for Positive X side of AubieSat.

\begin{tabular}{llll}
\hline & Unmixed vector & \multicolumn{2}{c}{ Material } \\
\hline 0.48 & 0.44 & Solar Cell 2 (AubieSat) \\
0.3 & 0.36 & Green Circuit Board (AubieSat) \\
0 & 0.20 & Red Wire with Kapton (MSU Sat) \\
0.21 & 0 & Grey Anodized Aluminum (MSU Sat) \\
& 0.097 & & \\
& & Vector Norm Approximation Error \\
\hline
\end{tabular}

The positive $\mathrm{x}$ side of AubieSat is the side that has the largest difference between the unmixed spectrum and the original mixed spectrum for the AubieSat at a nearly $10 \%$ estimated error. Comparing the two spectra, the aluminum frame is 
Chapter 3 - Spectral Unmixing | 73 the major difference between the two component vectors. This is due to three different phenomena: first, as noted above the side is covered by an epoxy or other clear resin whose spectrum is unknown, which is filtering the light that hits the solar cells, creating a prominent set of organic absorption features which also appear in the kapton tape used. The second is expected due to the BRDF of aluminum; aluminum is highly specular, and falls off rapidly away from the principle reflection axis. This, added to the geometry of the spacecraft will tend to reduce the presence of the aluminum. Third, the orientation and variation of BRDF between the materials likely plays the largest role in the variation between the estimated area proportions and the unmixed proportions. Without knowledge of how each material responds to changes in orientation (the shape of the BRDF off-axis) and for the purposes of this research it is not practical to attempt to change the estimated orientation to acquire a closer match. Any attempt to do so would be multiplying by the correct value to change the material proportion to be closer to what it ought to be, without a background meaning.

Comparing these results to the negative y side of the same spacecraft, which is shown in Figure 35 below. It is important to note, the components are present in the same arrangement and proportions, but it lacks the clear resin coating of the positive $\mathrm{x}$ side.

Comparing the unmixing results, the lack of the unaccounted for material provides a much closer inverted spectrum, shown in Figure 36 and Table 6. 


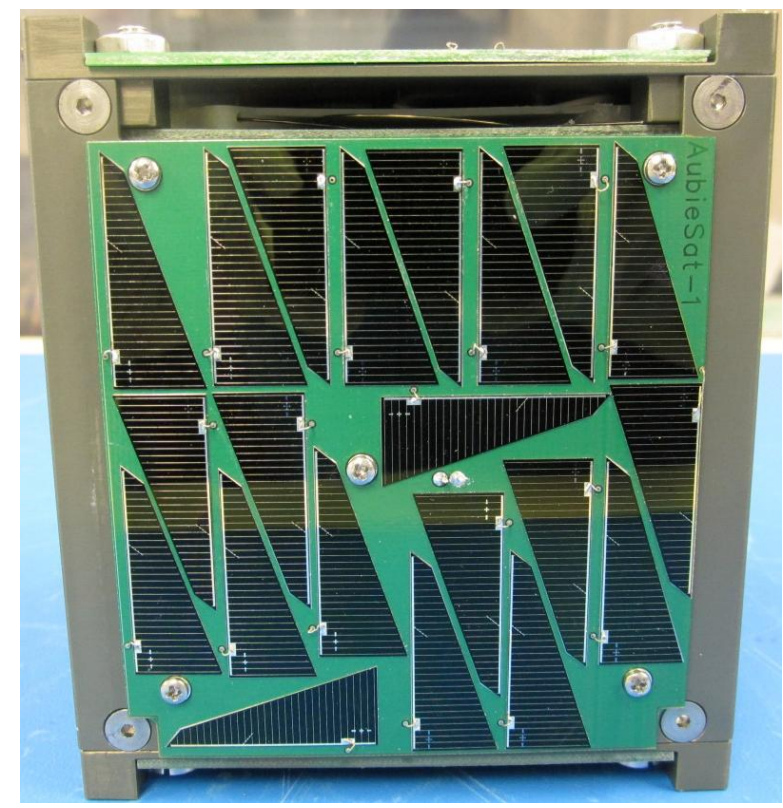

Figure 35. Negative y side of the AubieSat cubesat.

Note the major difference between this and the positive $x$ side, which is the lack of the clear resin coating.

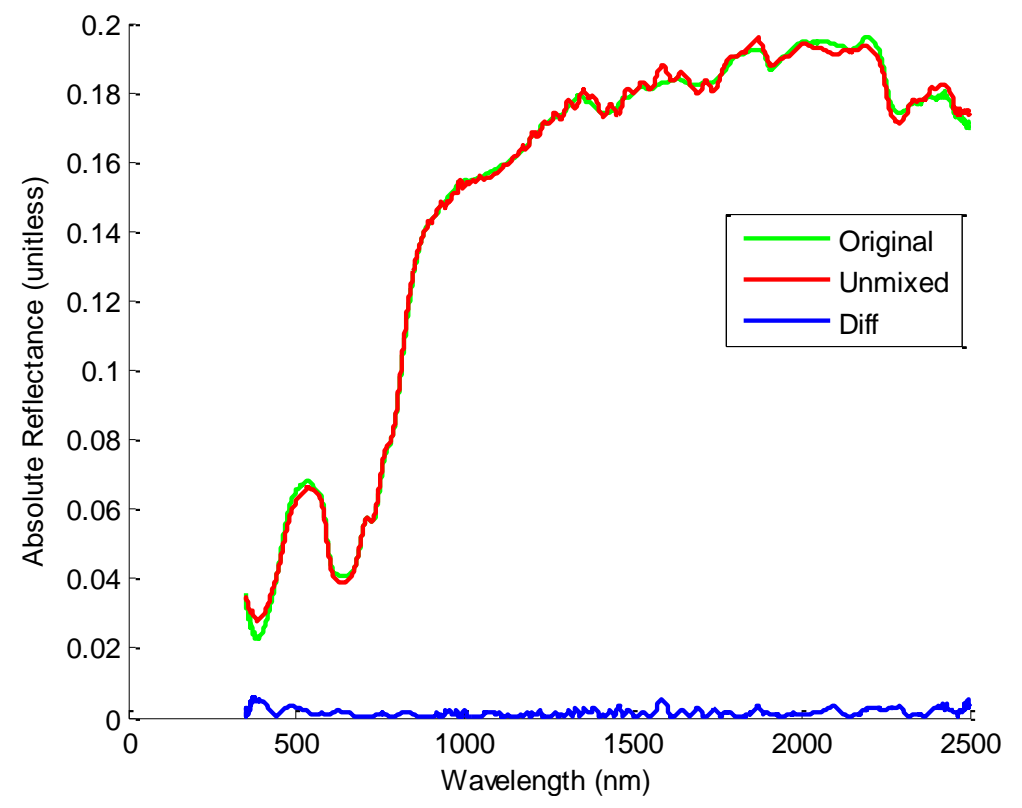

Figure 36. AubieSat Negative $Y$ unmixing results.

Shown above is the combined compared to the inverted spectrum for the negative y side of the AubieSat. Note that the overall shape between the two is very similar, while the unmixed spectrum possesses the sinusoidal feature common to certain types of solar cells. 
Table 6. AubieSat Negative $\mathrm{Z}$ unmixing results.

\begin{tabular}{llll}
\hline Estimated area & \multicolumn{4}{l}{ Unmixing results: } \\
\hline 0.48 & 0.227786 & Solar Cell 1 & (AubieSat) \\
0 & 0.000311 & Solar Cell 2 & (AubieSat) \\
0 & 0.011414 & Green Circuit Board $\quad$ (AubieSat) \\
0.3 & 0.586222 & Uncoated Green Board (AubieSat) \\
0 & 0.027313 & Red Wire & (MSU Sat) \\
0.21 & 0.085711 & Grey Anodized Aluminum (MSU Sat) \\
& & & \\
& 0.011687 & Vector Norm Approximation Error \\
\hline
\end{tabular}

Summing the three major types of materials (assuming that the variation of materials is due to a linear combination of the material appearances from orientation differences, and the material with the highest proportion is the actual material present), the solar cell has a $26 \%$ presence, the circuit board has a $62 \%$ presence, and the anodized aluminum has an $8 \%$ presence. The remaining $4 \%$ is found in the red wire, which is likely representative of some of the materials present on the spacecraft which were not included in the unmixing because of the small proportion of such materials on the spacecraft.

As in the previous case, it is believed that the major differences between the material spectra are due to the major differences in material BRDF between the various components. Based on observations of the materials the solar cells and aluminum have a very 'spikey' BRDF, with most reflected light being specularly reflected. Circuit boards, however, tend to have a more diffuse reflectance, with a more distributed BRDF. Off-axis the circuit board will tend to have a higher value 


\section{Chapter 3 - Spectral Unmixing |76}

than the other components, while close to the axis, the aluminum and solar cells will tend to have a higher inverted proportion. This effect can be remedied by taking the individual and combined spectra at very close to the same orientation, however for remote observations this cannot be achieved easily on most objects of interest.

The positive y side of MSU's HRBE provides a much better example for unmixing, as it contains two different types of circuit board, white uncoated and green uncoated. Given what can be assumed to be similar BRDF values for each material, the balance of those materials ought to be close to the approximate composition. The reference photograph is shown in Figure 37 below.

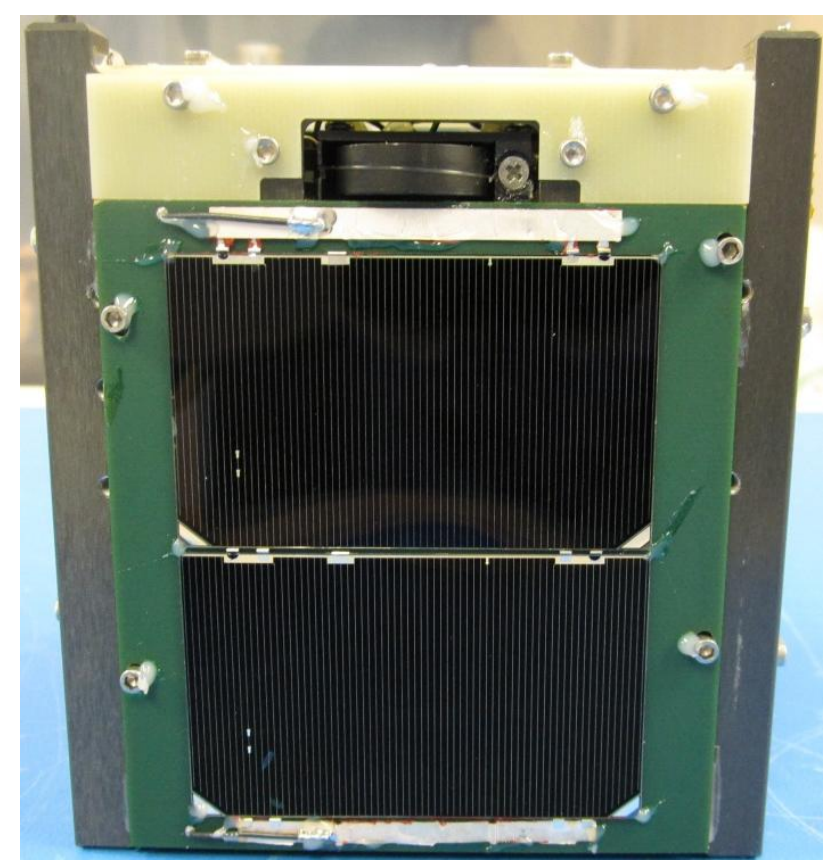

Figure 37. HRBE Positive $\mathbf{Y}$ reference photograph.

Two different types of circuit board are visible, white uncoated and green uncoated. 
Figure 38 and Table 7 show the results of unmixing the combined spectrum for the negative y side of HRBE.

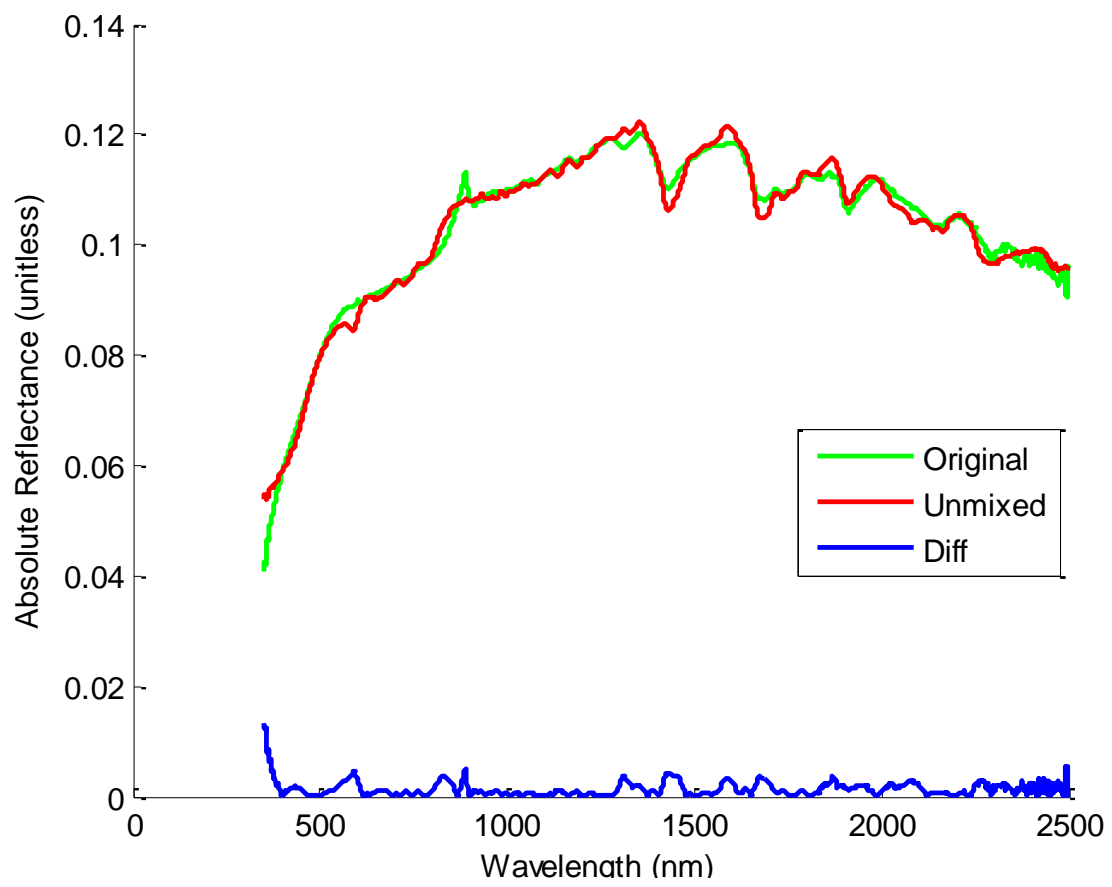

Figure 38. HRBE Positive $Y$ side unmixing results.

Shown above are the unmixing results for negative y side of HRBE. Important fea tures to note, there's a shark tooth near 890nm not present in the unmixed spectrum, and an absorption feature near $590 \mathrm{~nm}$ not present in the original spectrum.

Table 7. HRBE Positive Y side unmixing results.

\begin{tabular}{llll}
\hline Estimated area & \multicolumn{4}{l}{ Unmixing results: } \\
\hline 0 & 0.089 & Solar Cell 1 & (AubieSat) \\
0 & 0.15 & Uncoated Green & Board (AubieSat) \\
0.48 & 0 & Solar Cell 3 & (MSU Sat) \\
0 & 0.28 & Red Wire & (MSU Sat) \\
0.20 & 0 & Green Circuit Board (MSU Sat) \\
0.13 & 0.22 & White Circuit Board (MSU Sat) \\
0.153 & 0.031 & Grey Anodized Aluminum (MSU Sat) \\
0.016 & 0.21 & Antennar r (MSU Sat) \\
0 & 0.019 & Solar Cell, no sinusoid feature \\
\hline & & & \\
\hline
\end{tabular}


Again, the fit between the unmixed approximation and combined spectrum is quite good, with only a $1.8 \%$ difference between the two spectra. There are a few major departures from the measured combined spectrum composition, however. The addition of a large proportion of what should be non-present red wire, the larger than expected presence of the antenna spectrum, the lack of solar cell and relatively non-present aluminum frame which should be present. Each of these is not necessarily expected, but is explainable. The solar cells and aluminum both suffer from the rapid change in BRDF off-axis. The red wire spectrum lacks significant features other than a plateau beginning at about $610 \mathrm{~nm}$. This is close in appearance to both the white circuit board and green circuit board, and is likely responsible for the depth of the absorption features. This is likely due to the background of the red wire when the spectrum was taken, as the red wire is in front of both white and green circuit boards, so a slight similarity in spectra is expected.

The over-representation of the antenna is somewhat expected, as the antenna itself is a shiny material, and is rolled up in the side of the spacecraft. This circular shape coupled with the shininess of the material gives rise to the overrepresentation of the antenna spectrum in this set.

The reference photograph for the positive y side of HRBE is shown below in Figure 39. The solar cells and aluminum are significantly over-represented, shown in Figure 40 and Table 8, which demonstrates the same sensitivity to the rapid change in BRDF off-axis. 


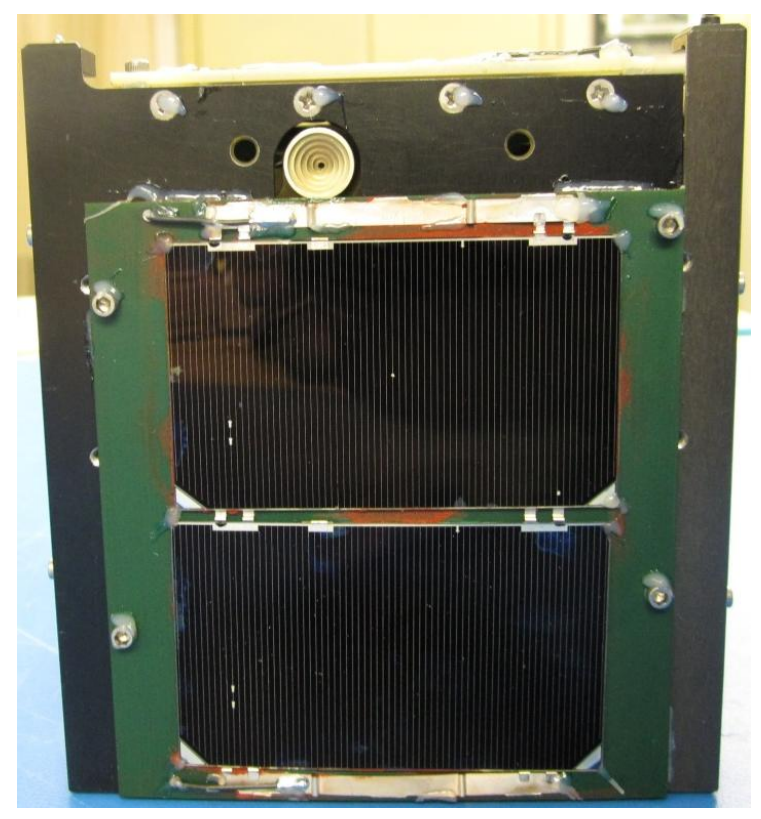

Figure 39. HRBE Negative $Y$ side reference photograph.

Note the similarities between this and the positive y side shown in Figure 37. HRBE Positive $Y$ reference photograph.. The main difference being the lack of white circuit board, and the lack of the antenna slot.

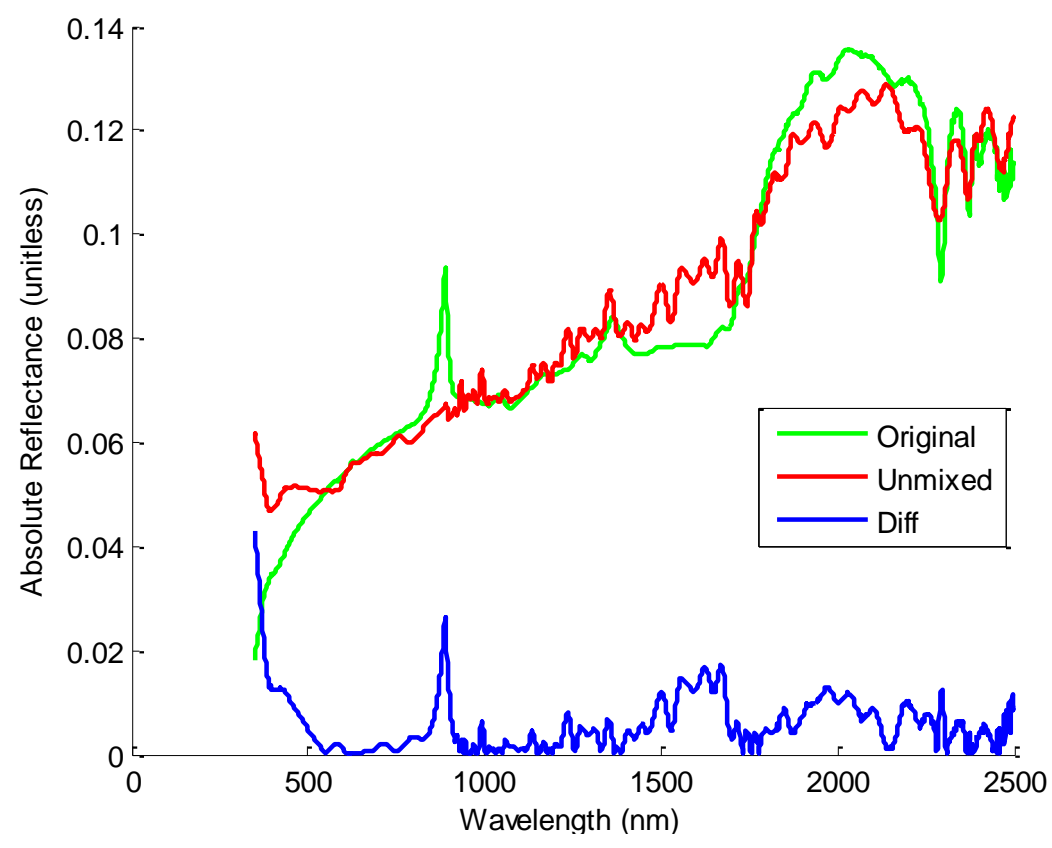

Figure 40. HRBE Negative $\mathrm{Y}$ unmixing results.

Note that this is one of the more poorly matched spectra, though the general shape and several major features are duplicated reasonably well. 
Chapter 3 - Spectral Unmixing | 80

Table 8. HRBE Negative $Y$ unmixing results.

\begin{tabular}{llll}
\hline Estimated area & \multicolumn{4}{l}{ Unmixing results: } \\
\hline 0 & 0.02 & Solar Cell 1 & (AubieSat) \\
0.48 & 0.77 & Solar Cell 2 & (AubieSat) \\
0 & 0 & Green Circuit Board (AubieSat) \\
0 & 0.051 & Uncoated Green Board (AubieSat) \\
0 & 0.058 & Red Wire & (MSU Sat) \\
0.13 & 0 & Green Circuit Board (MSU Sat) \\
0.26 & 0.16 & Grey Anodized Aluminum (MSU Sat) \\
& & & \\
& 0.082 & Vector Norm Approximation Error \\
\hline
\end{tabular}

There are a couple of features of this unmixing result to note, first the strong representation of a linear combination of solar cell spectra, and the presence, though not as strong as it ought to be, of the grey anodized aluminum. From these two, we can conclude that the aluminum's BRDF is somewhat more concentrated around the specular spike than the solar cell's BRDF. Again, it is likely that the inclusion of the red wire spectra here is as a fix to the circuit board spectrum, (which is needed to correct for a change in orientation).

\section{5 - IUS Remote Observation}

The final test was attempting unmixing on a spectral data set taken remotely. The IUS rocket bodies were a two-stage booster used to boost satellites into GEO or planetary orbits by NASA. A set of spectral observations were made by the NASA AMOS Spectral Study (NASS). Spectral matching was performed via inspection, and 
modeling the expected composition of the spacecraft, and the composition was determined with reasonable accuracy based on comparison to the measured spectrum and the known composition of the rocket body pre-flight [13] A reference picture of the IUS rocket body is shown below in Figure 41:

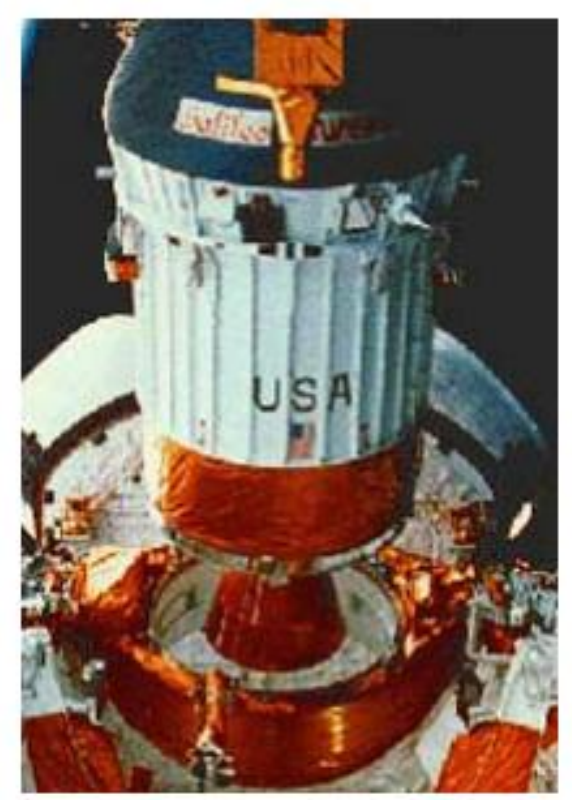

Figure 41. Picture of an IUS with both stages in the space shuttle bay (courtesy of NASA) [13]

Given a set of spectra for all the expected spacecraft materials on the IUS rocket bodies, the remote measured spectra were unmixed successfully. Getting a true confirmation of the success of unmixing is difficult on a remote observation, unless a combined spectrum and composition information is taken before launch, and the orientation of the spacecraft is known, or accounted for in the model. The full results are presented in Appendix B. There were two main identifiable spectra, first the spectrum presented in Ref [13] with a relatively flat region above 410nm, 


\section{Chapter 3 - Spectral Unmixing | 82}

and a steep curve beginning at around 350nm (shown in Figure 42), second a fairly curved spectrum ranging from $400 \mathrm{~nm}$ to $730 \mathrm{~nm}$ (shown in Figure 44)

The remote data is varied in the wavelength discretization where data was taken, and was not over the same range of wavelengths as the wavelengths. To perform vector mathematics for unmixing it was necessary to interpolate (using the spline function in MATLAB) to rescale the basis and input measured spectrum to the appropriate wavelengths for unmixing, so that an appropriate comparison could be made. Figure 38 and Table 9 display the results for unmixing the first IUS spectrum.

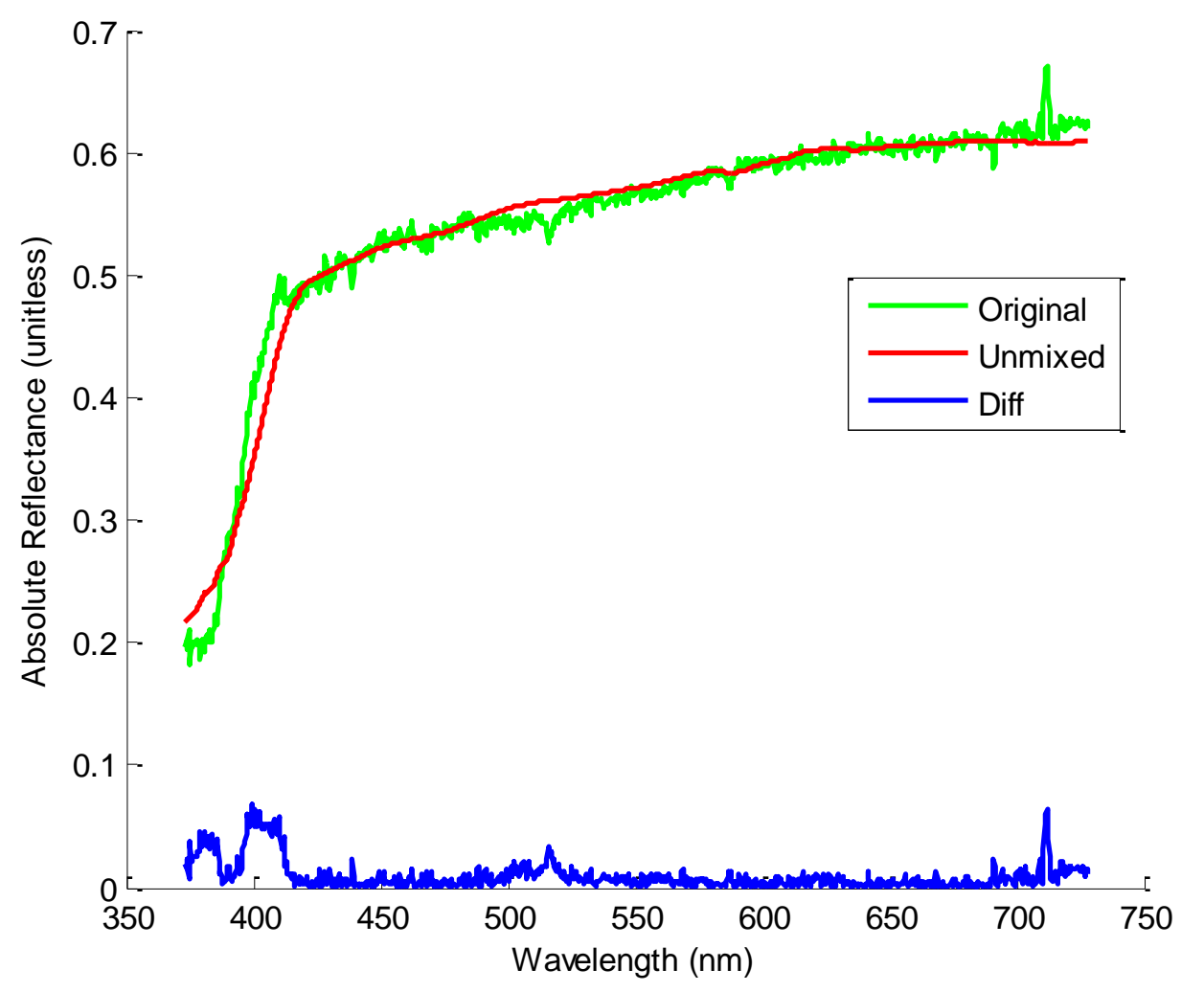

Figure 42. IUS Rocket Body spectra measurement (Measurement 1) unmixing results comparison. 
Table 9. IUS Rocket Body Measurement (Measurement 1)

\begin{tabular}{ll}
\hline Proportion & Material \\
\hline 0 & Aluminum holder \\
0.55 & Carbon Epoxy Nozzle \\
0 & IUS23 Corrugated upper section \\
0.26 & IUS23 Midsection white paint \\
0 & IUS23 Upper section white paint \\
0.05 & Blue cable \\
0 & MLI gold \\
0.14 & MLI gold - back \\
0.026 & Vector Norm Approx. Error \\
\hline
\end{tabular}

There are in total 8 measurements of what appears to be the same orientation of the IUS rocket body, with the same basica shape to the spectrum. The unmixed values for each of these (presented in Appendix B) were all based around the same 4 materials, Carbon Epoxy Nozzle, White paint, Blue Cable (always very small in proportion, likely used for its shape for small corrections), and the MLI gold - back. This choice of materials agrees with the materials in Ref [13], though the proportions selected are somewhat different. The original conclusion from the paper was that the MLI thermal control material was damaged or removed during the engine firing, and is verified by this unmixing, showing a lack of the original MLI spectrum, but $\sim 13 \%$ presence of the backside of the material. Additionally, the white paint appears to be less present than the nozzle, suggesting an orientation with the nozzle pointed towards the observation, perhaps on an relative to the optical axis, as shown below in Figure 43: 


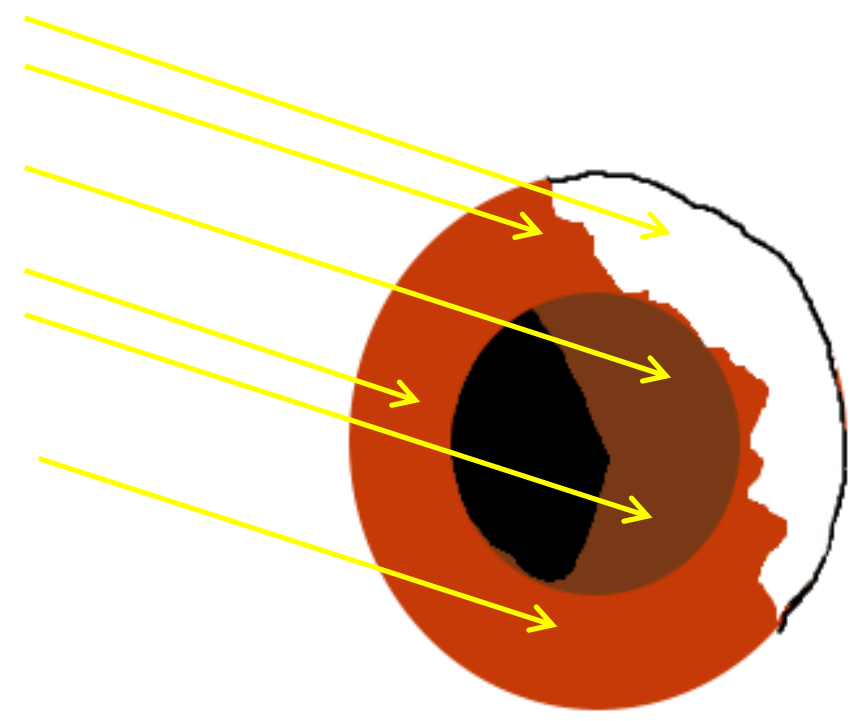

Figure 43. Possible orientation of the IUS rocket body in Measurement 1

The most likely orientation of the incident light is coming in at an angle, presenting the light to the spectrometer with a more significant portion from the nozzle, with relatively low reflectance from the MLI, due to its highly specular reflective characteristics, along with some light reflected from the white paint. Thus presenting the nozzle as the primary portion, and the other materials as secondary characteristics.

The other spectrum which had defining features was a measurement made between $400 \mathrm{~nm}$ and $730 \mathrm{~nm}$, shown in Figure 44 and Table 10 below is the unmixing of this measurement: 


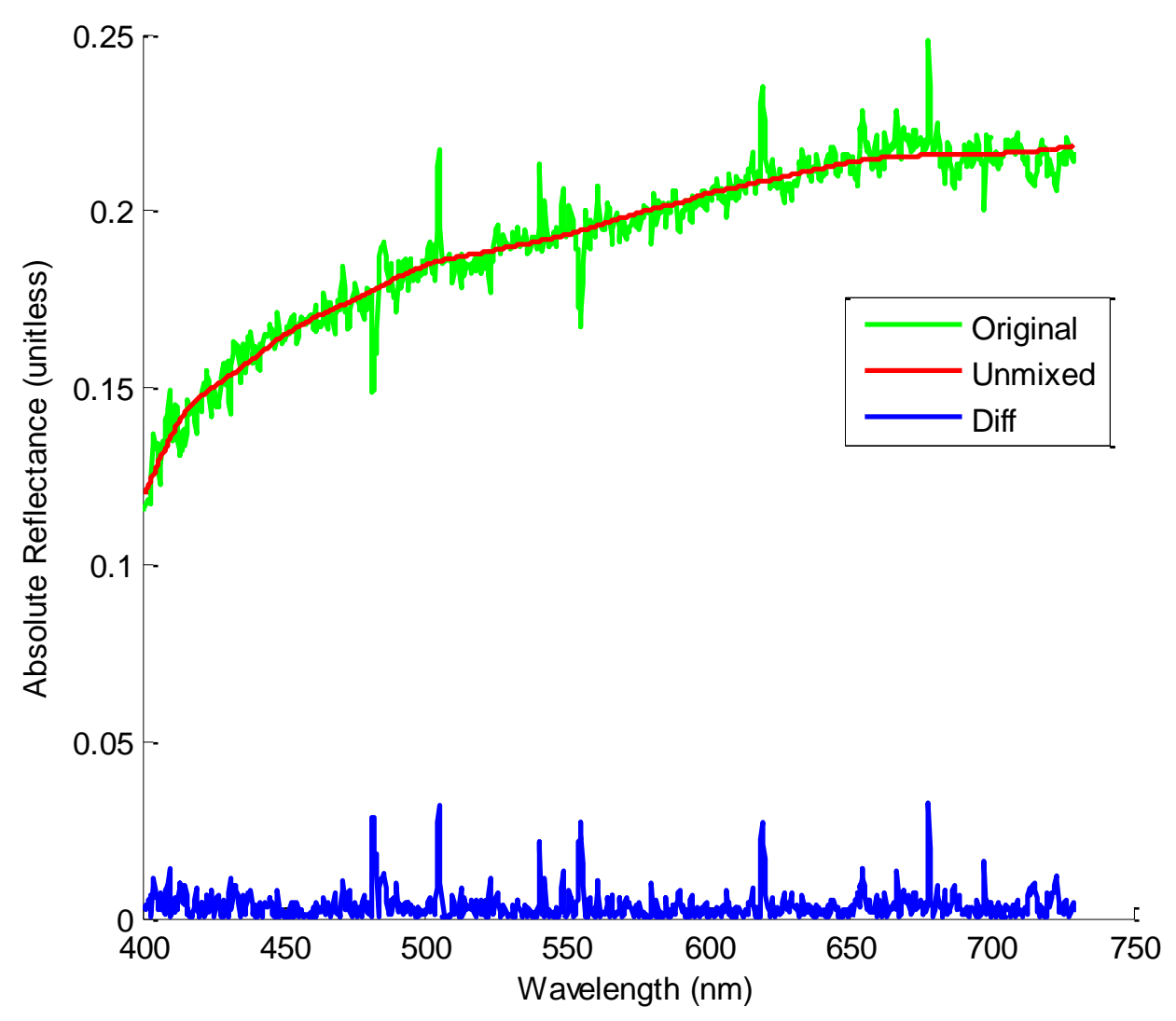

Figure 44. IUS Rocket Body spectra (Measurement 2), unmixing results comparison.

Table 10. IUS Rocket Body Measurement (Measurement 2)

\begin{tabular}{ll}
\hline Proportion & Material \\
\hline 0.20 & Aluminum holder \\
0.31 & Carbon Epoxy Nozzle \\
0 & IUS23 Corrugated upper section \\
0.09 & IUS23 Midsection white paint \\
0 & IUS23 Upper section white paint \\
0.13 & Blue cable \\
0.07 & MLI gold \\
0.18 & MLI gold - back \\
& \\
0.027606 & Vector Norm Approx. Error \\
\hline
\end{tabular}

Again, the fit is very close, with a similar set of materials shown, with a few key differences. First, there are two more materials present, or at least included in 
Chapter 3 - Spectral Unmixing | 86

the unmixing: the Aluminum holder (which displays typical aluminum features) and a small presence of the front side of the MLI gold. This measurement is likely from a different angle, with less nozzle than the previous measurement. However, without the presence of distinct features, such as the $\sim 800 \mathrm{~nm}$ from aluminum, or even features such as the knee present in Figure 42 above, it is difficult to fully confirm the presence of the aluminum predicted by this unmixed set of materials.

\section{6 - Unmixing applied to ID of outgassed materials}

An application of the unmixer is the identification of single materials, which have been changed slightly. For a small set of candidate materials this seems an over-application, but if there were a very large set of candidate spectra, comparing manuall quickly becomes tedious. To this end, an interesting test case is to check the performance of the unmixer in identifying materials which have outgassing effects (and eventually other effects) based on non-outgassed basis spectra. This is particularly informative for those cases where a component on a spacecraft may not yet have a measured space environment spectrum.

The first spectrum tested, shown in Figure 45. 2mil Kapton x Aluminum (Kapton Side) Unmixing Identification.Figure 45 and Table 11, is an unmixing identification of outgassed 2mil Kapton x Aluminum (Kapton side). 


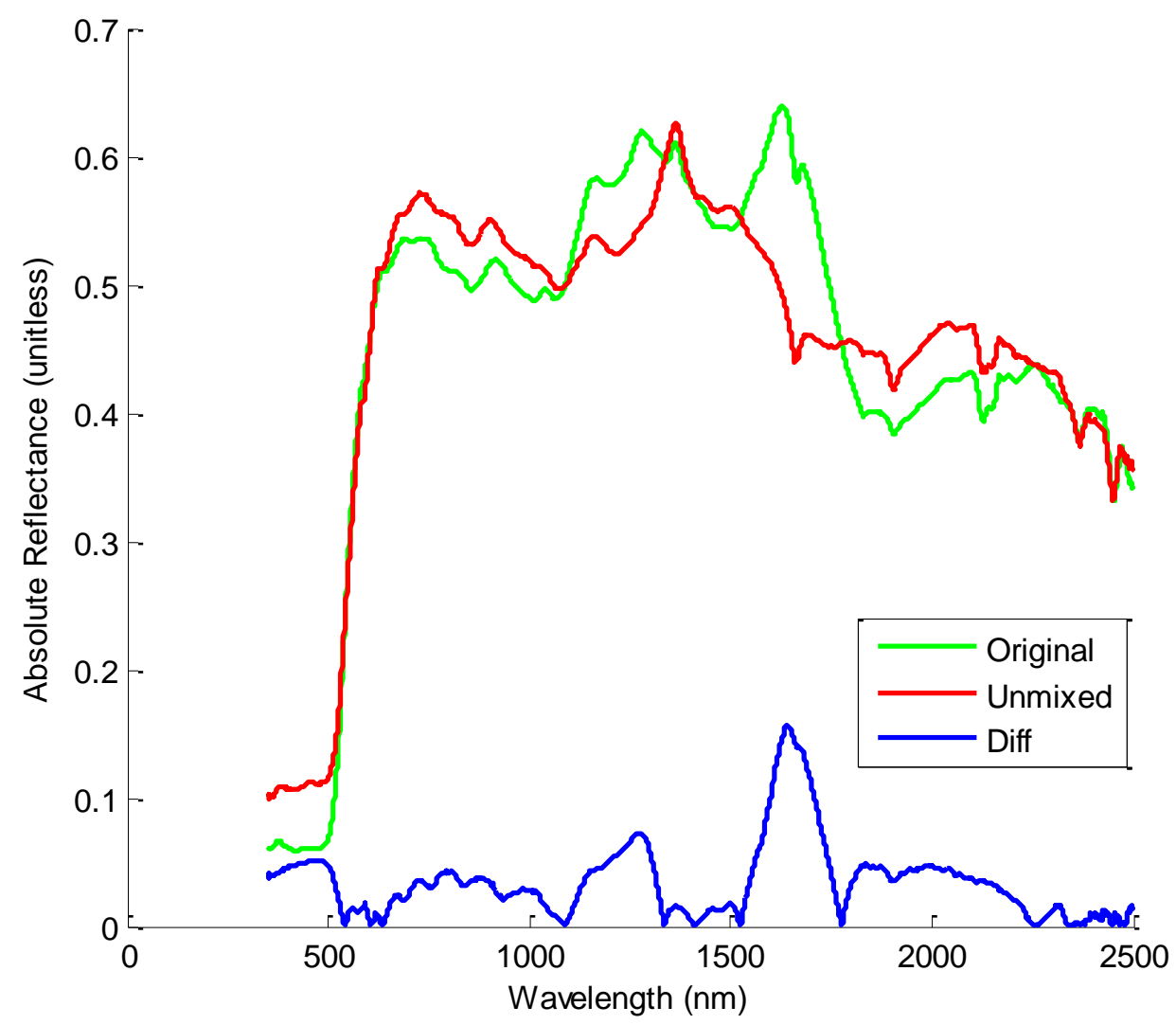

Figure 45. 2mil Kapton x Aluminum (Kapton Side) Unmixing Identification.

Shown above is the unmixing of an outgassed Aluminum $x$ Kapton sample based on a set of nominal (not outgassed) spectra.

Table 11. 2mil Kapton x Aluminum (Kapton Side) Unmixing Identification.

\begin{tabular}{rl}
\hline $\begin{array}{c}\text { Estimated } \\
\text { Proportion }\end{array}$ & Material \\
\hline 0 & Aluminum Beta Cloth (Al) \\
0 & Aluminum Beta Cloth (Cloth) \\
0 & Germanium x Black Kapton (Ge) \\
0.15 & Teflon x Silver Inconel \\
0.85 & 2 mil Kapton x Aluminum (Kapton side) \\
& \\
0.10 & Vector Norm Approximation Error \\
\hline
\end{tabular}


Chapter 3 - Spectral Unmixing | 88

Note that the major errors are the features that were noted in chapter 2 that appear in outgassing. Considering the numerical output, the largest material proportion is the correct material it is likely that this method will produce correct results on an unknown input spectrum, as long as the correct individual pre-outgas measurement exists in the basis of spectra.

The second example shown is Aluminum Beta cloth, which as seen in Chapter 2, does not experience measurable outgassing effects. However, there were some slight differences in the spectrum between the nominal and outgassed spectrum (shown inFigure 27) and the unmixer attempts to rectify this to provide a closer fit with small amounts of the other basis spectra provided. This is shown in Figure 46 and Table 12 below. Note a couple of phenomena, first the gross majority of the estimated present spectrum is Aluminum Beta Cloth, which successfully identifies the material. As noted above, the small differences are due to the slight orientation difference between pre and post outgas measurements, and the unmixer is attempting to correct for those differences, and does so, comparing Figure 20 and Figure 46, the between the two spectra is much smaller, so as to be nearly nonexistent, as evidenced by the $0.2 \%$ difference between the two spectra. This sort of error should be eliminated in a model where the orientation of a material, and the variations in spectrum and magnitude can be effectively accounted for. 


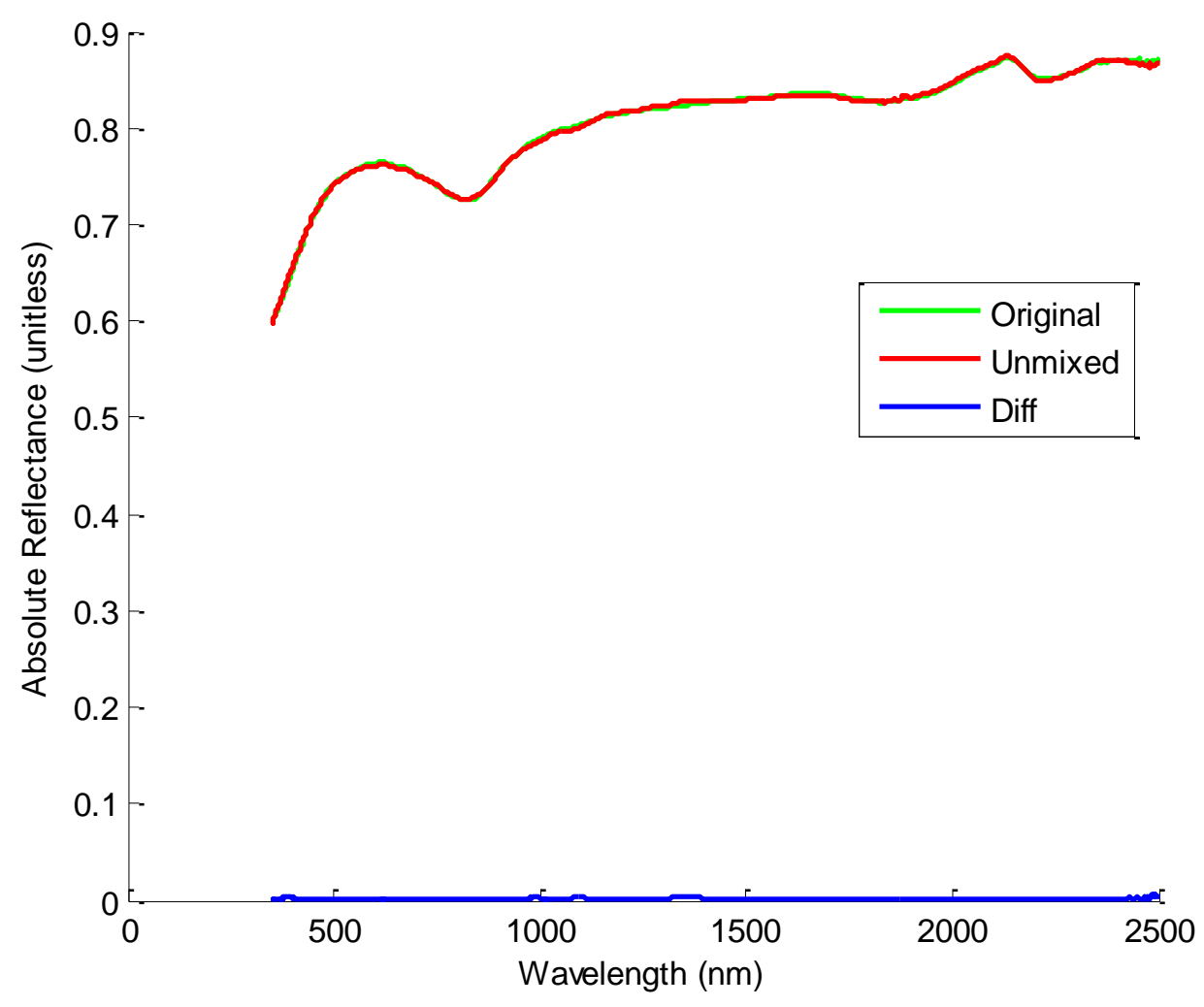

Figure 46. Aluminum Beta Cloth (Aluminum Side) Unmixing Identification.

Shown above is the unmixing of an outgassed Aluminum Beta Cloth (Aluminum side) sample based on a set of nominal spectra.

Table 12. Aluminum Beta Cloth (Aluminum Side) Unmixing Identification.

\begin{tabular}{rl}
\hline $\begin{array}{c}\text { Estimated } \\
\text { Proportion }\end{array}$ & Material \\
\hline 0.93 & Aluminum Beta Cloth (Al) \\
0.05 & Aluminum Beta Cloth (Cloth) \\
0 & Germanium x Black Kapton (Ge) \\
0.02 & Teflon x Silver Inconel \\
0.0001 & 2 mil Kapton x Aluminum (Kapton side) \\
& \\
0.002 & Vector Norm Approximation Error \\
\hline
\end{tabular}




\section{7 - Unmixing Conclusions}

As was laid out in chapter 1, this direct linear unmixing as was performed here is at best an intermediate step to full unmixing, taking BRDF into account. As such, there can be some significant differences between the material proportions calculated by the unmixing algorithm and the true material proportions. In general, the largest difference between the unmixed spectrum and combined original spectrum for lab measurements was $13.5 \%$, with some spectra having a as little as a $1.6 \%$ difference between the unmixed spectrum and the original combined spectrum. This relatively small difference leads to the conclusion that unmixing is occurring correctly, with work needed on including BRDF in the unmixing.

Another point of interest in improving the results, a better system for acquiring basis spectra for unmixing, rather than the system employed on the CubeSats displayed here, where the materials were measured directly on the CubeSat (which can allow for the inclusion of background materials), the better practice would be to separate the spacecraft materials and measure against a black background. This was not an option on AubieSat and HRBE, but allowed for a proof of concept measurement set.

One phenomenon noted in the unmixing results was a mixture of various types of similar materials. This has to be caused by one of two effects. The first option is on these materials there is a 'specular spectrum' and a 'diffuse spectrum' that are linearly mixed depending on angle, and the measurements made on the 
Chapter 3 - Spectral Unmixing | 91

materials in different conditions represent two different combinations of these spectra. These combinations then show up in the final unmixing results to best approximate the partial combination in the original mixed spectrum. This may vary from material to material, some measurements made of specular reflectance spectra are very well correlated with their non-specular counterparts, where on other materials (such as the solar cells measured in chapter 2) there are significant differences between the two cases. The other option is that the mixed spectra are indicative of a non-perfect fit, and need to be weeded out in a two-step process. Calculating the unmixed proportions, and comparing the possible types under the assumption that each material spectrum is distinct, and one will prove a better fit than the others. With this method, categories would be chosen, and the best single material in each category would be chosen. This would break down if multiple types of a single material appear, such as on HRBE where both white and green circuit boards appear on the same side.

The final note is that in a region with no major features, such as the second IUS measurement presented (Figure 44), an estimation of the materials may return a very close match, but without features to compare depth and location in the unmixed model, it is difficult to check the veracity of the output. This is an unfortunate side effect of using a limited region of spectrum for measuring, as fewer features will show up in the measurements. 


\section{4 - Conclusions}

\section{1 - Lessons Learned}

There were a number of lessons learned from the work performed on this project, many of which will be critical to future work on developing the spectral knowledge of space environment effects on spacecraft materials, as well as the mathematical processes used to identify materials and proportions. The first addressed here are those lessons learned while measuring space environment effects. Full recommended procedures can be found in Appendix C. But the most important part of taking measurements for comparison of pre/post effects is minimizing the role that variation in orientation and position relative to the measurement device and light source plays on the material spectrum. This can be alleviated by taking measurements on two samples side by side, one with exposure to the desired space environment effect, one without, and making a comparison. Other possible solutions involve more complex setups to measure the exact position of each component (sample, light source, optical probe) for each measurement, but this would be tedious for each measurement, and likely difficult / expensive to set up. Such a solution would, however, be beneficial in conjunction with a set of BRDF measurements, or as a tedious means of taking such BRDF measurements.

Another lesson learned in the spectral measurements was an initial measurement of the time a sample can be out of the chamber before the space environment effects begin to fade (in this case, only performed on the outgassing 
effects). However, the tests showed that for the materials tested, within roughly 20 minutes there was little change in the effects. Further testing on this is suggested, however, especially for longer durations out of the vacuum chamber, as well as for other space environment effects.

The last lesson learned on the space environment effect measurements was the relatively significant role that the surface condition played in the changes measured. On many of the film substrates, the substrate material exhibited outgassing effects that are likely due to changes in surface materials, rather than absorbed material being outgassed. This is also recommended as an effect for future investigation.

Researching and performing the unmixing work provided several lessons. First, as mentioned earlier, the work presented here is only a partial solution to the unmixing problem and needs the inclusion of an orientation model to be a full unmixing model. One of the major lessons is that, similar to other unmixing models (though this unmixer can handle more input endmembers than others) the accuracy of the prediction can be hindered by the number of input endmembers. Though not fully necessary for the scope of this project, it will become a problem with future work with a much larger database of materials. 


\section{2 - Conclusions}

The goals at the outset were to take spectral measurements of some space environment effects on spacecraft materials, and to create an unmixing algorithm capable of unmixing basic spectral proportion in mixed spectra. Development of these and other methods of identification are important to the characterization of the objects in orbit around our planet. Specifically, by acquiring material knowledge of the objects in orbit, a much more accurate estimation of the mass and size of objects in orbit can be made, increasing the accuracy of models estimating the state of the debris environment, among other benefits. Both of the original goals of this thesis were met, with lessons learned for future work and improvements to the methodology to acquire these measurements.

Spectral measurements were made on materials in three conditions, first measurements made in normal atmospheric condition as a generic basis for identification. Space environment effects were measured in a typical vacuum environment and a single measurement of the effects of Atomic Oxygen (AO) erosion were made (single measurement only due to the availability of materials and facilities). Overall, most materials experience very little change due to simple exposure to the vacuum chamber, the most significant changes are due most likely to changes in the surface chemistry or materials, and the outgassing of absorbed gasses and water in the material, which is fairly predictable in materials whose spectra in normal atmospheric conditions are known. On the AO effects, the erosion 
seems to have removed some surface characteristics of the Kapton film measured, providing what is perhaps a more 'pure' spectrum for Kapton film. These effects will require further investigation to verify their occurrence and causation.

An investigation was made on the feasibility of calculating true material proportion from mixed spectra and a method was developed to do partial unmixing,

under the assumption that material BRDF was relatively constant across all components. This assumption proved to be incorrect, but allowed for unmixing based on the shape of the individual spectra while being inaccurate on the magnitude of the components. The shape of the unmixed spectrum output was accurate to within $13 \%$ on all unmixed spectra, and within $6 \%$ on most sets.

\section{3 - Future Work}

On future work, the major direction that needs to be explored, and was explored briefly in this report, is the effects of measurement orientation on the spectrum of a material. Depending on the material, orientation may make a significant difference in shape, not only magnitude. An apparatus that would help to solve this problem would also solve the problem encountered in many of the outgassing measurements, of keeping orientation consistent between the before and after measurements. The first piece of equipment would be a two-segmented arm with some means of recording its location relative to its mounting location. 
Something as simple as a modified drafting arm, capable of measuring angles on each of its joints would be sufficient. Or a double-jointed camera arm mount measured relative to some corners of the measurement table. The other piece of equipment would be a device for holding samples is important, especially the film materials measured in many of these experiments. Something along the lines of a double clip holder used in soldering electronic components would be valuable, though an apparatus with four clips to appropriately hold a material still, perhaps rubber coated to avoid damaging the material surface.

On the unmixing algorithm, an investigation of the possible linear combination of specular and diffuse spectra for a material, or a possible characterization of materials, allowing for a more refined unmixing. The major improvement, however, will lie in the inversion of the BRDF values. To do this, two things are needed: BRDF measurements for each spacecraft materials, and a modification to the unmixing algorithm. As was discussed in Chapter 3, the unmixing algorithm developed here unmixes to find the $p_{i} B_{i}$ term for each component below:

$$
S_{\text {combined }}=\sum_{i=1}^{n} p_{i} B_{i} S_{i}+N
$$

Where $p_{i}$ is the true material proportion, and $B_{i}$ is the BRDF for the material in the mixed measurement orientation. Given unmixing solves for this value, and it's been shown that assuming that $B_{i}$ is approximately constant across the various 
materials is not accurate, $B_{i}$ must be calculated individually. With knowledge of the individual material BRDF (an array of values, or equation defining these values) it should be possible to calculate the true material proportion using some linear algebra, or other optimization techniques. 


\section{Bibliography}

[1] Scientific and Technical Subcommittee of the United Nations Committee on the Peaceful Uses of Outer Space, "Technical Report on Space Debris," United Nations, New York, 1999.

[2] T. W. Thompson, R. M. Goldstein, C. D. B., S. E. G. and A. E. J. Potter, "Radar detection of centimeter-sized orbital debris: Preliminary Arecibo observations at 12.5-CM wavelength".

[3] K. M. Jorgensen, "Using Reflectance Spectroscopy to Determine Material Type of Orbital Debris," University of Colorado, Boulder, 2000.

[4] A. C. Tribble, The Space Environment, Princeton, New Jersey: Princeton University Press, 2003.

[5] G. Holister, R. Brackmann and W. Fite, "The use of modulated atomic-beam techniques for the study of space-flight problems," Planetary and Space Science, vol. 3, pp. 162-168, 1961.

[6] F. L. Pedrotti, L. S. Pedrotti and L. M. Pedrotti, Introduction to Optics, Third Edition, San Francisco: Pearson Addison Wesley, 2007.

[7] N. Keshava and J. F. and Mustard, "Spectral Unmixing," IEEE Signal Processing Magazine, no. January 2002, pp. 44-57, 2002.

[8] F. E. Nicodemus, "Directional Reflectance and Emissivity of an Opaque Surface," Applied Optics, vol. 4, no. 7, pp. 767-773, 1965.

[9] Griffiths, Introduction to Electrodynamics, 3rd Edition, Upper Saddle River, New Jersey: Prentice-Hall, 1999.

[10] D. Hall, "Surface Material Characterization from Multi-band Optical Observations," DTIC, Kihei, HI, 2010. 
[11] K. Jorgensen, A. Rivkin, R. Binzel, R. Whitely, C. Hergenrother, P. Chodas, S. Chesley and F. Vilas, [36.02] Observations of J002E3: Possible Discovery of an Apollo Rocket Body, Houston, 2003.

[12] K. J. Abercromby, J. Okada, M. Guyote, K. Hamada and E. Barker, "Comparisons of Ground Truth and Remote Spectral Measurements of the FORMOSAT and ANDE Spacecraft," 2006.

[13] K. Jorgensen-Abercromby, M. Guyote and J. Okada, "Inertial Upper Stage Surface Property Study," in The Fourth European Space Debris Conference, Darmstadt, Germany, 2005.

[14] R. N. Clark, G. A. Swayze, E. K. Livo, R. F. Kokaly, S. J. Sutley, J. B. Dalton, R. R. McDougal and C. A. Gent, "Imaging Spectroscopy: Earth and planetary remote sensing with the USGS Tetracorder and expert systems," Journal of Geophysical Research, vol. 108, no. 5131, p. 44, 2003.

[15] K. Luu, C. Matson, M. Giffin, K. Hamada, J. Lambert and J. Snodgrass, "Object Characterization from Spectral Data," AFRL/DEBI (Det 15), Kirtland AFB, 2003.

[16] J. Piper, V. P. Pauca, R. J. Plemmons and M. Giffin, "Object Characterization from Spectral Data Using Nonnegative Factorization and Information Theory," in AMOS Technical Conference, Maui, HI, 2004.

[17] V. P. Pauca, R. J. Plemmons, M. Giffin and K. M. Hamada, "Unmixing Spectral Data for Space Objects using Low-Rank Non-Negative Matrix Factorization," 2003.

[18] Q. Zhang, H. Wang, R. J. Plemmons and V. P. Pauca, "Tensor Methods for Hyperspectral Data Analysis: a space object material identification study," Journal of the Optical Society of America A, vol. 25, no. 12, pp. 3001-3012, 2008.

[19] T. Sato, "Shape Estimation of Space Debris Using Single-Range Doppler Interferometry," IEEE Transactions on Geoscience and Remote Sensing, vol. 37, no. 2, pp. 1000-1005, 1999.

[20] C. M. Pieters and E. M. Fischer, "Optical Effects of Space Weathering: The Role of the Finest Fraction," Journal of Geophysical Research, vol. 98, no. E11, pp. 
20,817-20,824, 1993.

[21] K. K. deGroh and B. A. Banks, "Investigation of Teflon FEP Embrittlement on Spacecraft in Low-Earth Orbit," Research and Technology, 1997.

[22] L. M. V. Martel, "PSRD - Space Weathering Agent: Solar Wind," 10 August 2009. [Online]. Available: http://www.psrd.hawaii.edu/Aug09/PSRDsolarwind.helium.pdf. [Accessed 23 May 2012].

[23] G. Elert and E. Al, "Wavelength of the Sun's Peak Radiation Output," The Physics Factbook, 2002. [Online]. Available: http://hypertextbook.com/facts/2002/TahirAhmed.shtml. [Accessed 15 July 2012].

[24] А. V. Borisov, "Doped quartz glass tube; grade КЛБ-3," Gusevskiy Glassworks, 2004. [Online]. Available: http://www.szd.rusglass.ru/en/product_08.html. [Accessed 18 July 2012].

[25] M. Bartholomew-Biggs, Nonlinear Optimization with Financial Applications, New York: Kluwer Academic Publishers, 2005.

[26] E. Cantu-Paz, Efficient and Accurate Parallel Genetic Algorithms, Norwell: Kluwer Academic Publishers, 2000.

[27] E. K. P. Chong and S. H. Zak, An Introduction to Optimization, New York: John Wiley \& Sons, Inc, 1996.

[28] G. Strang, Computational Science and Engineering, Wellesley: WellesleyCambridge Press, 2007. 


\section{Appendix A - All Spectra measured}

When multiple spectra are displayed, this is due to multiple measurements of a single material displaying variant features. The individual condition for each measurement was not recorded.

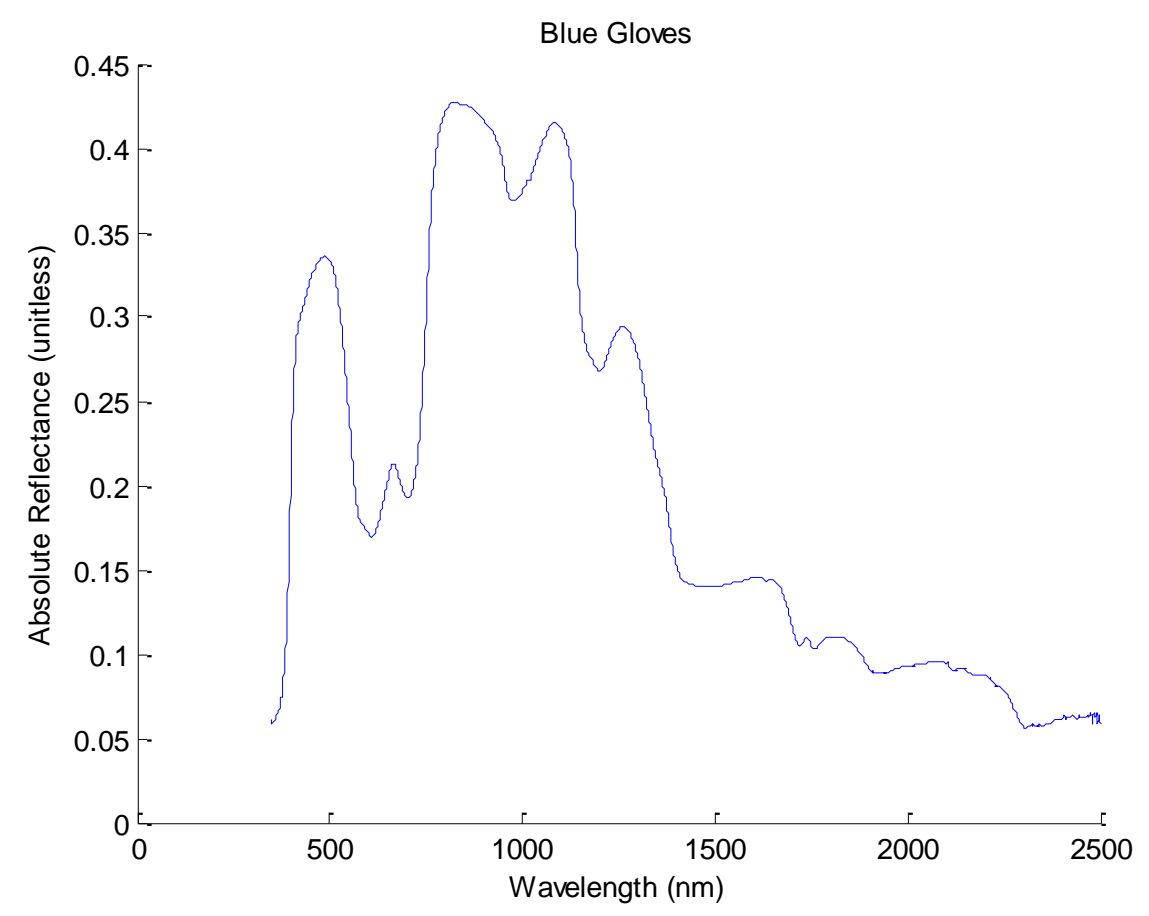



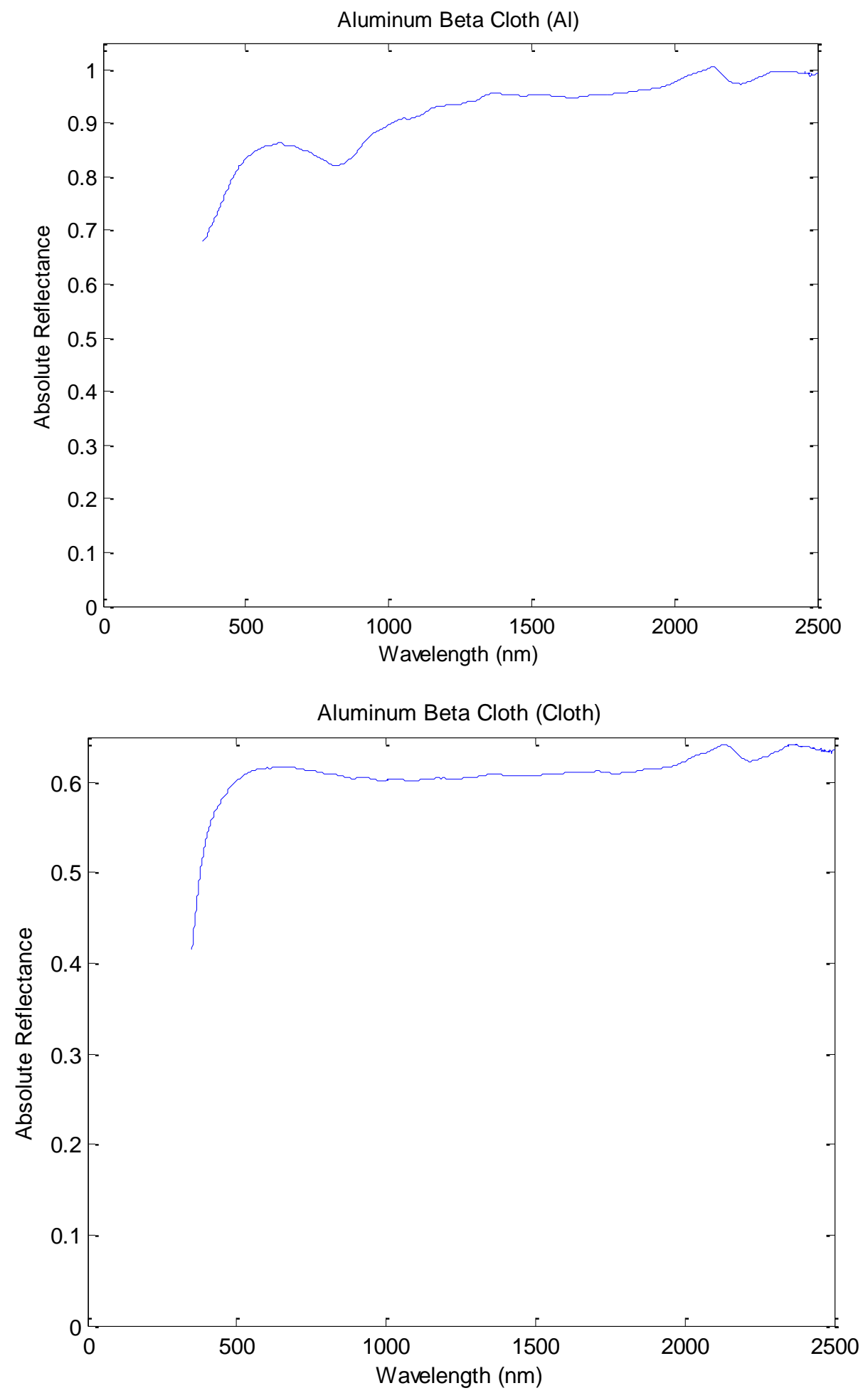
Appendix A |A3
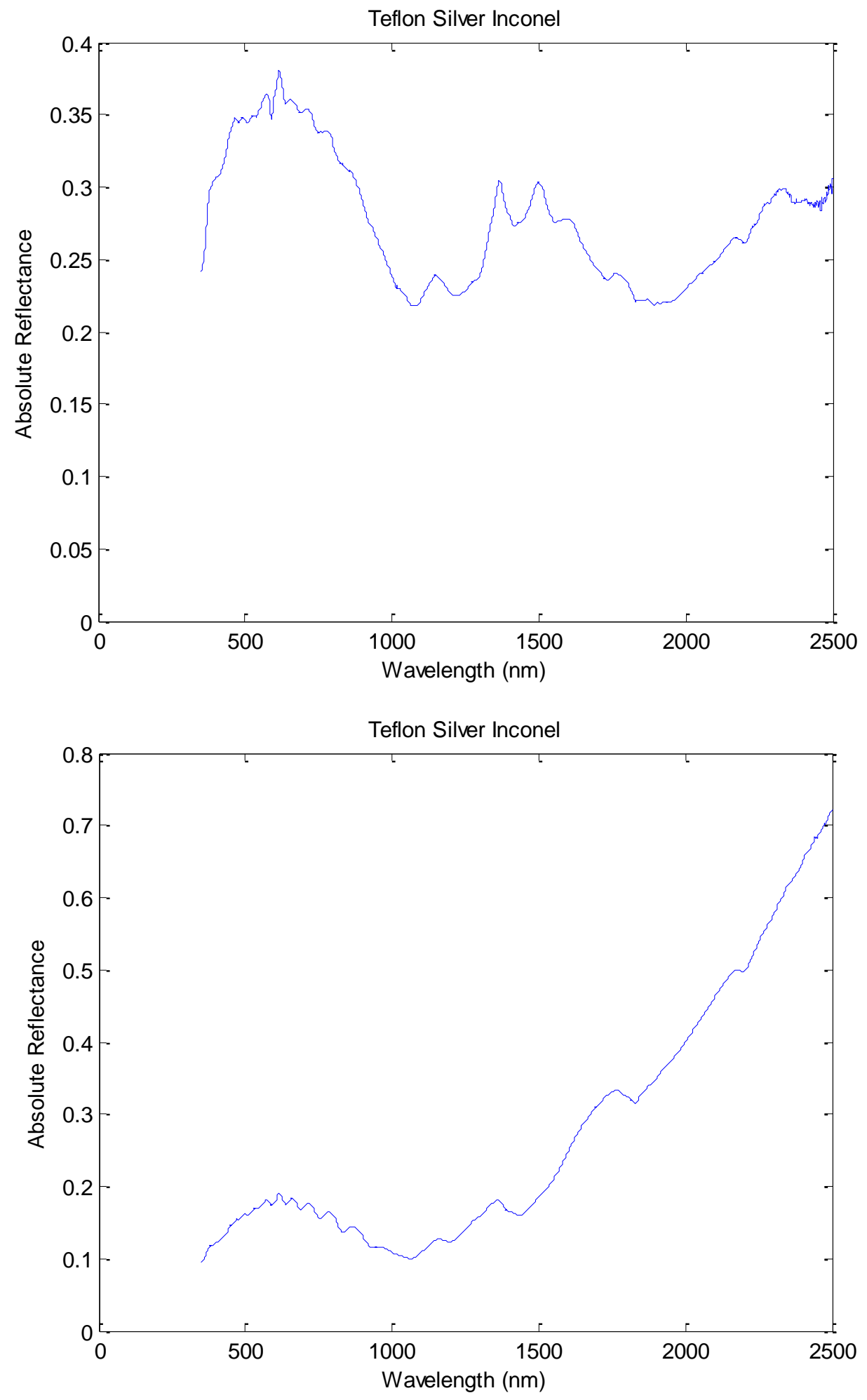

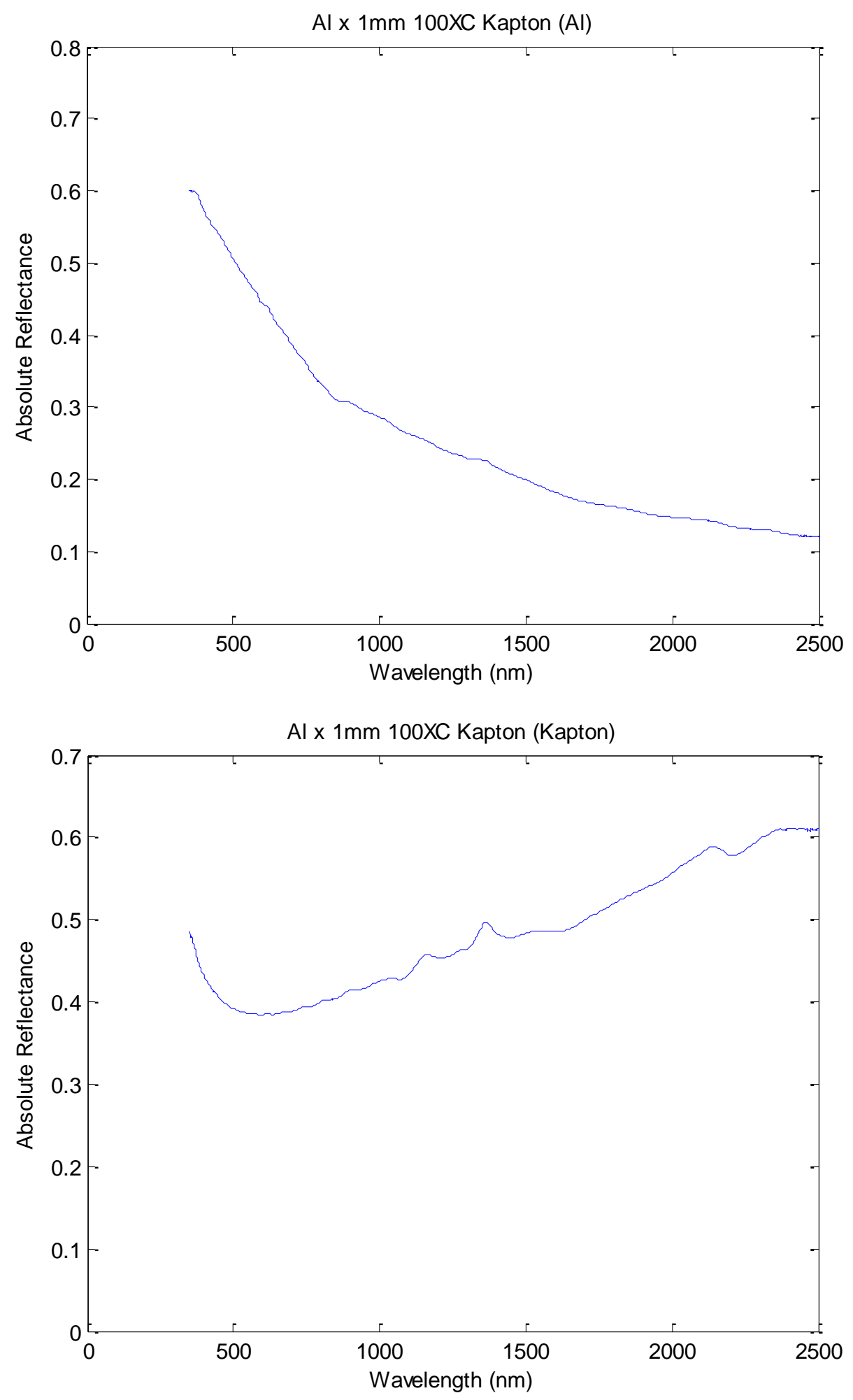

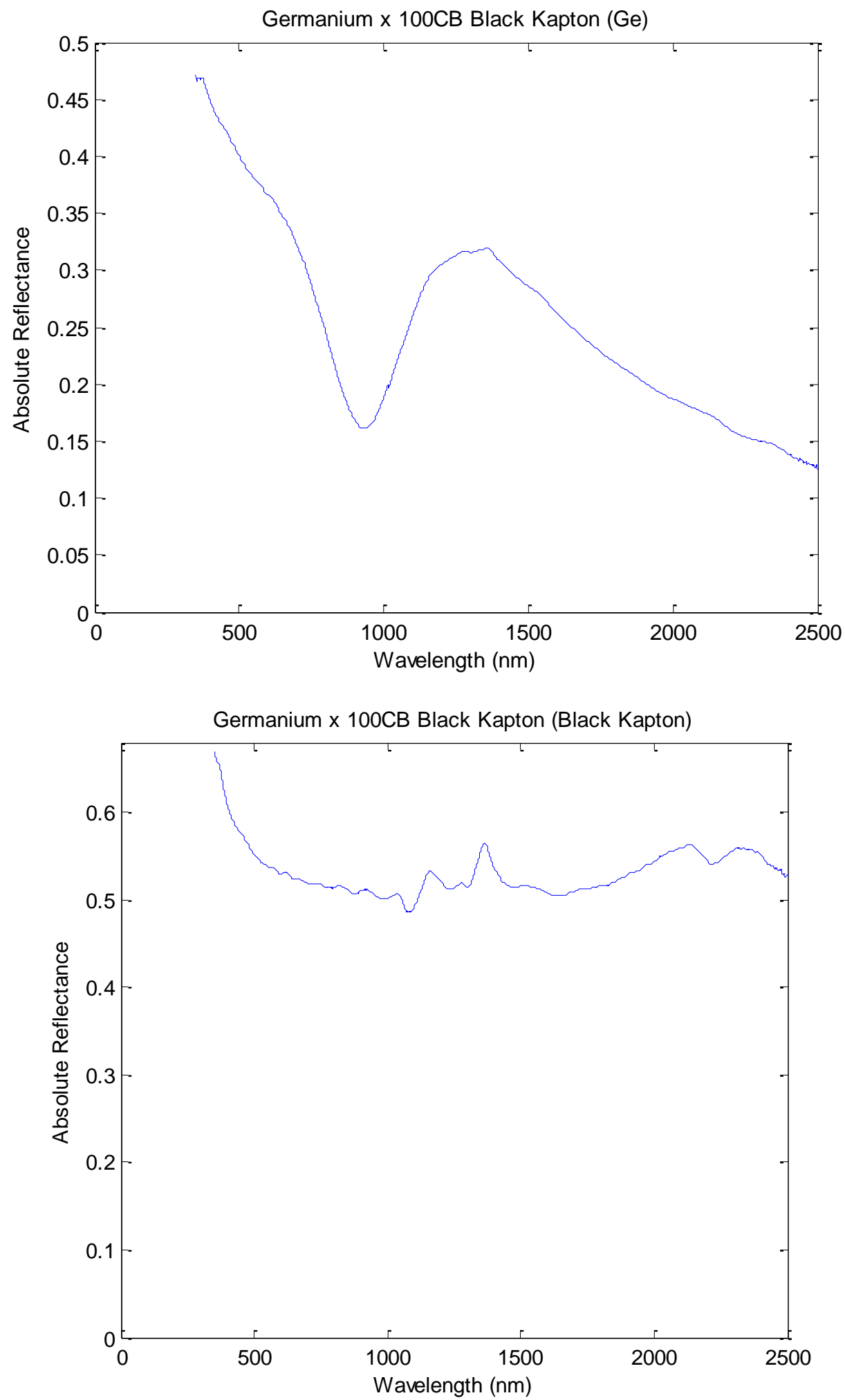
Appendix A |A6
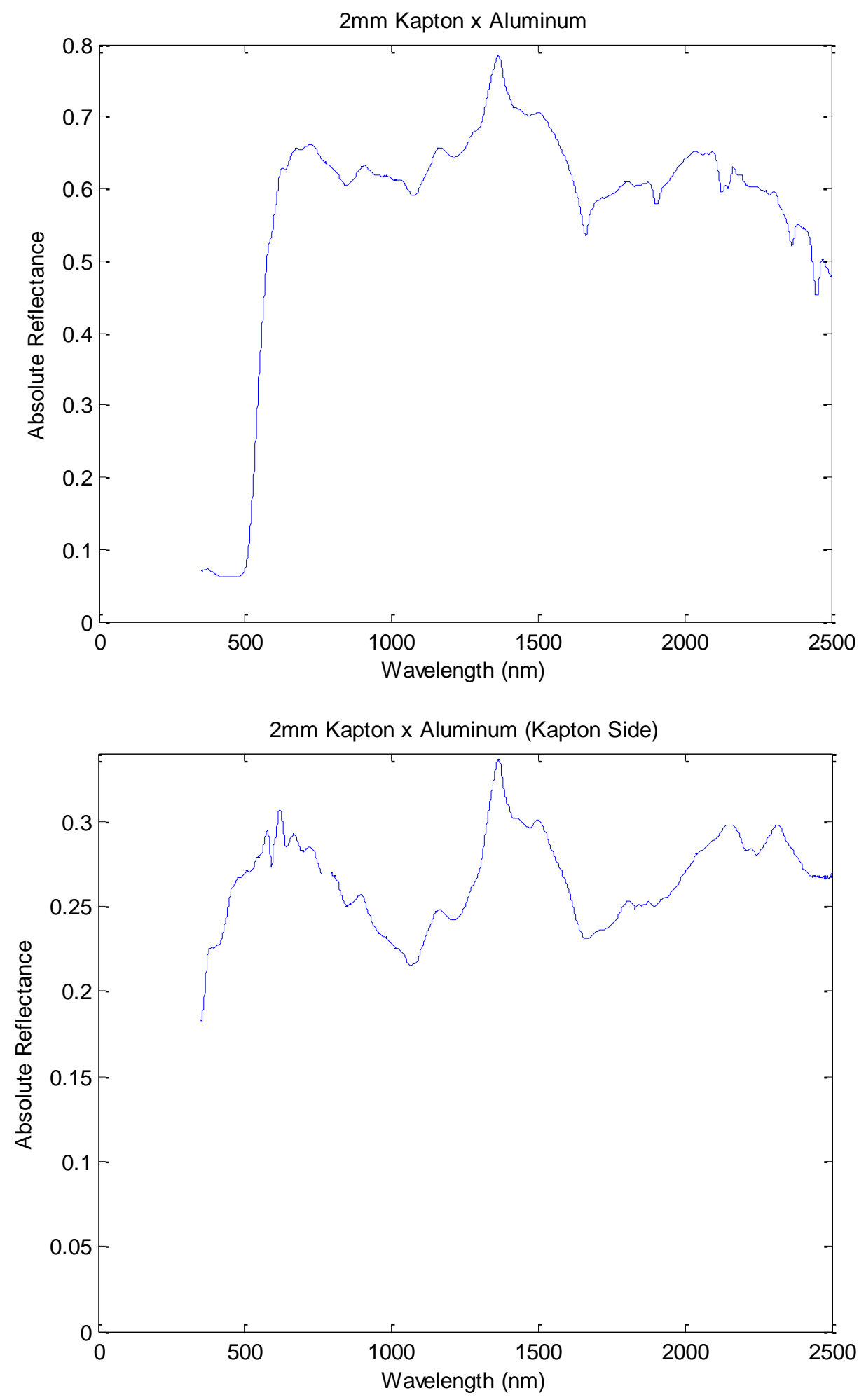

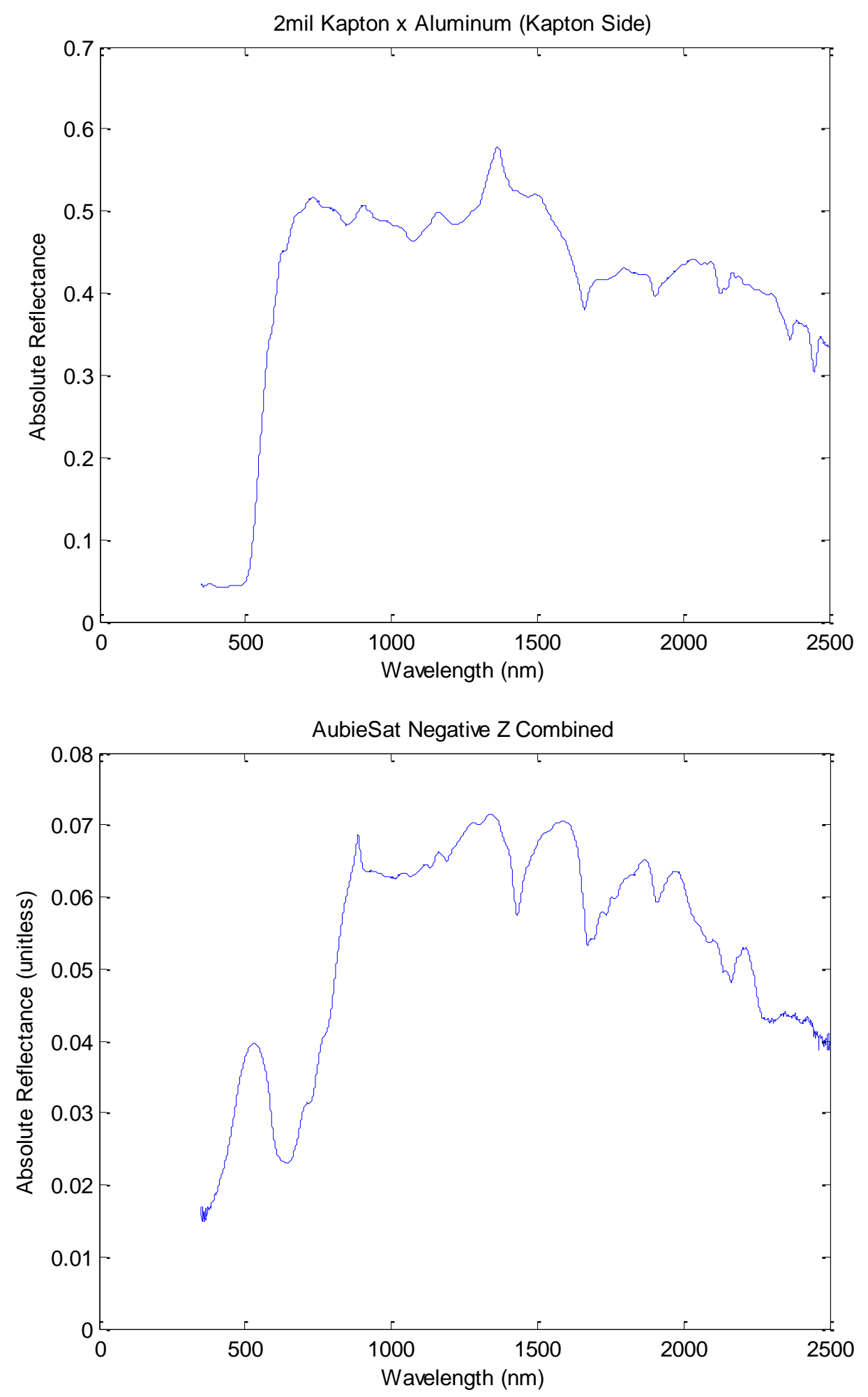
Appendix A $\quad$ A8
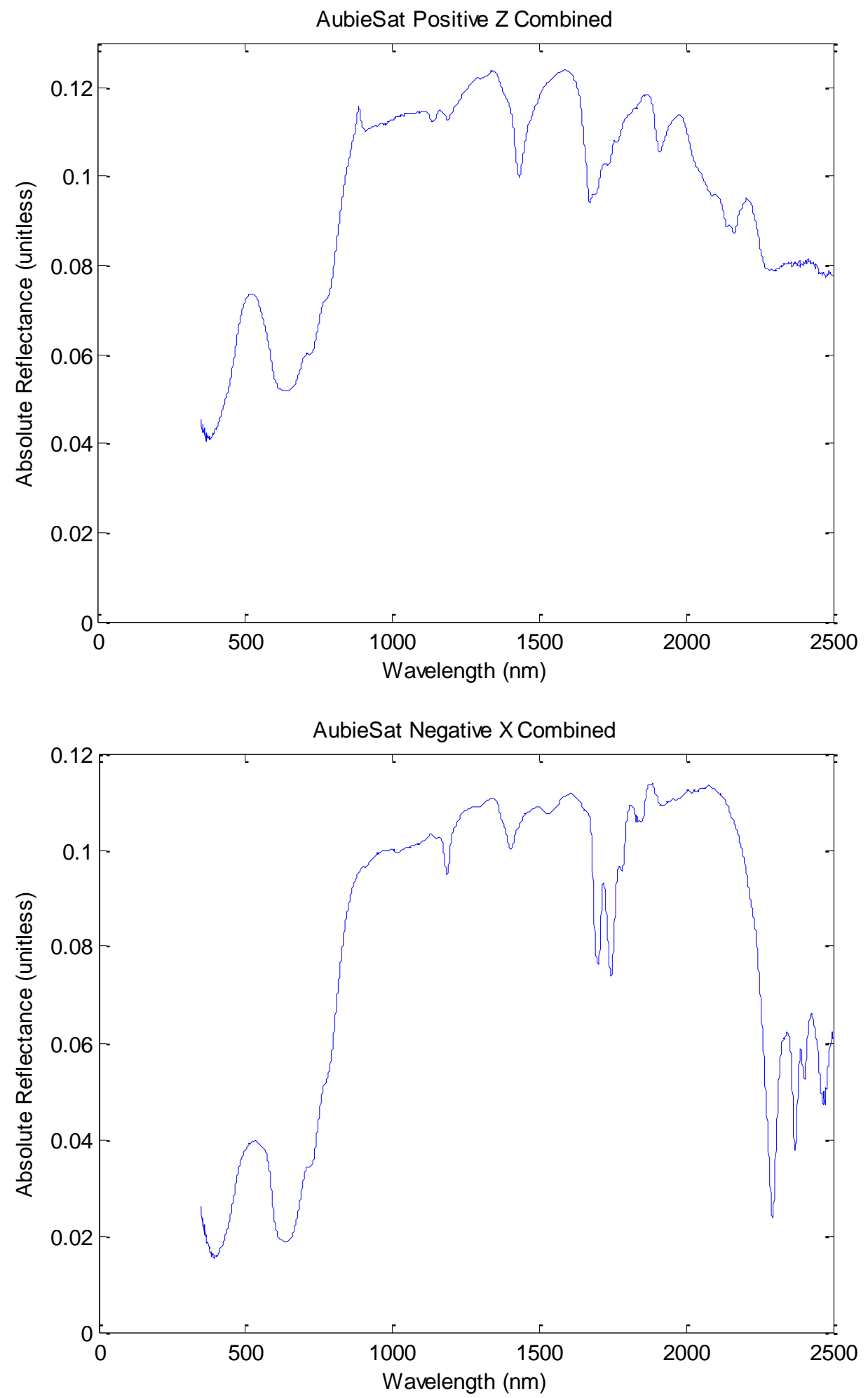
Appendix A |A9
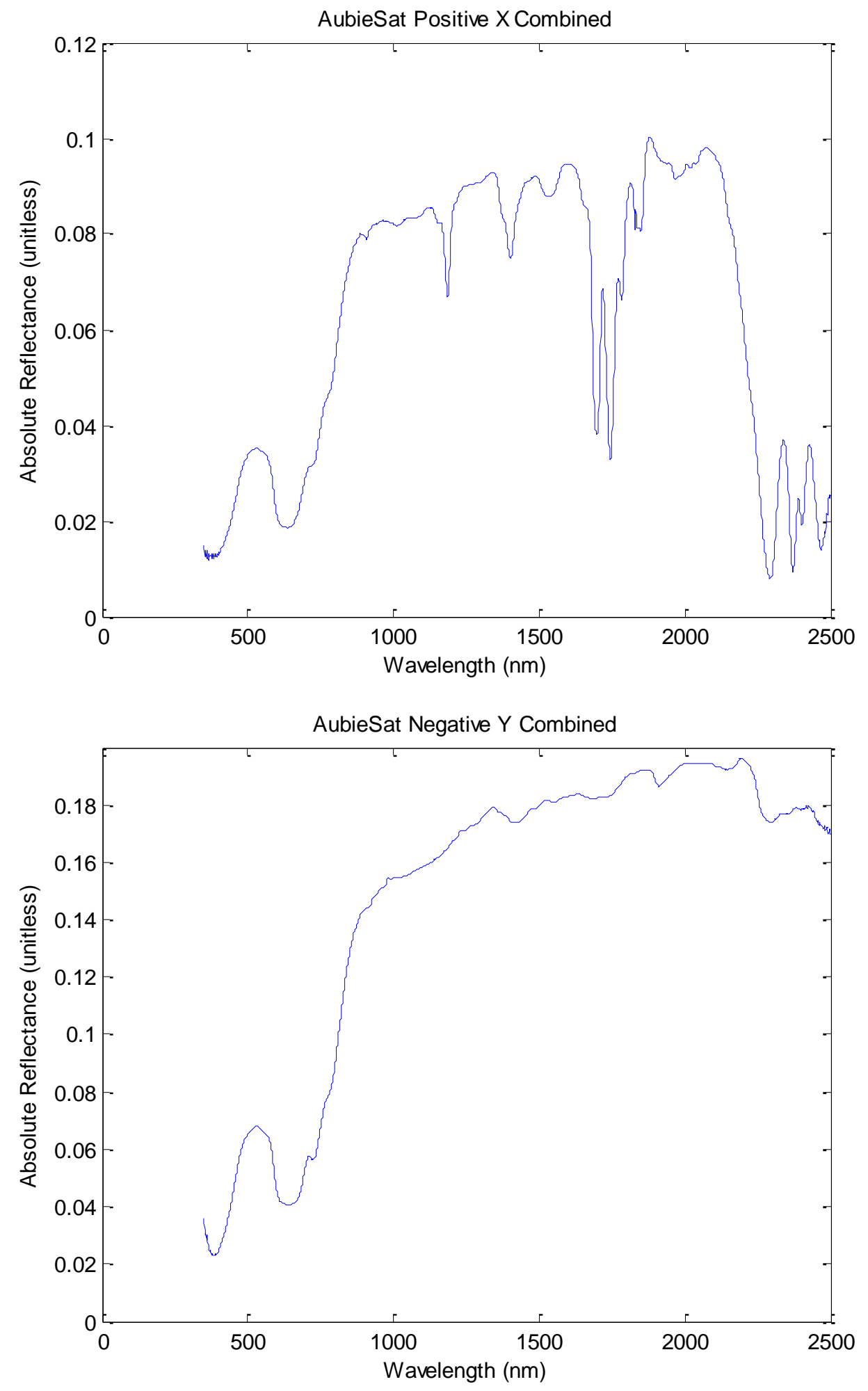
A p p endix A |A10
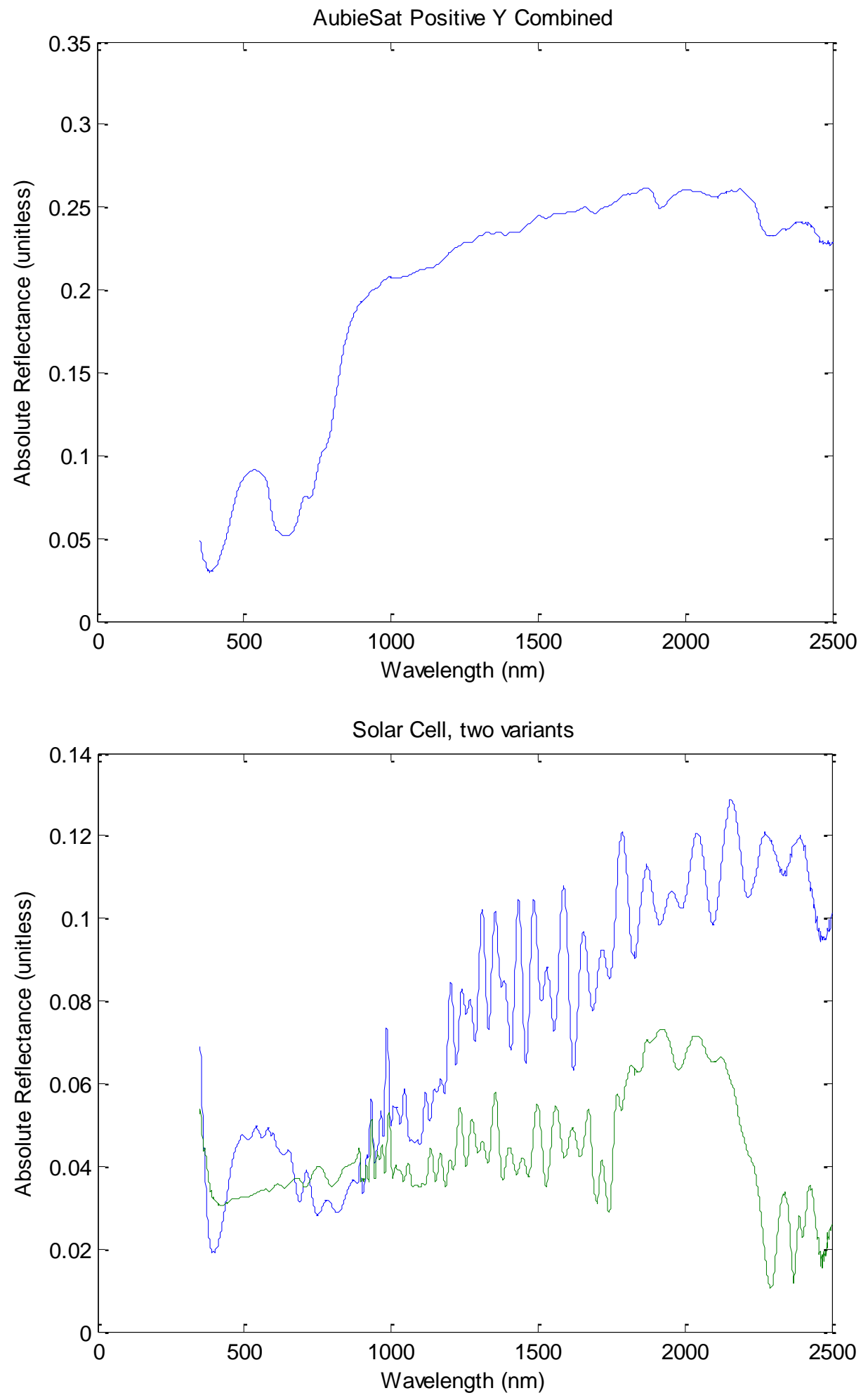
Appendix A $\mid$ A11
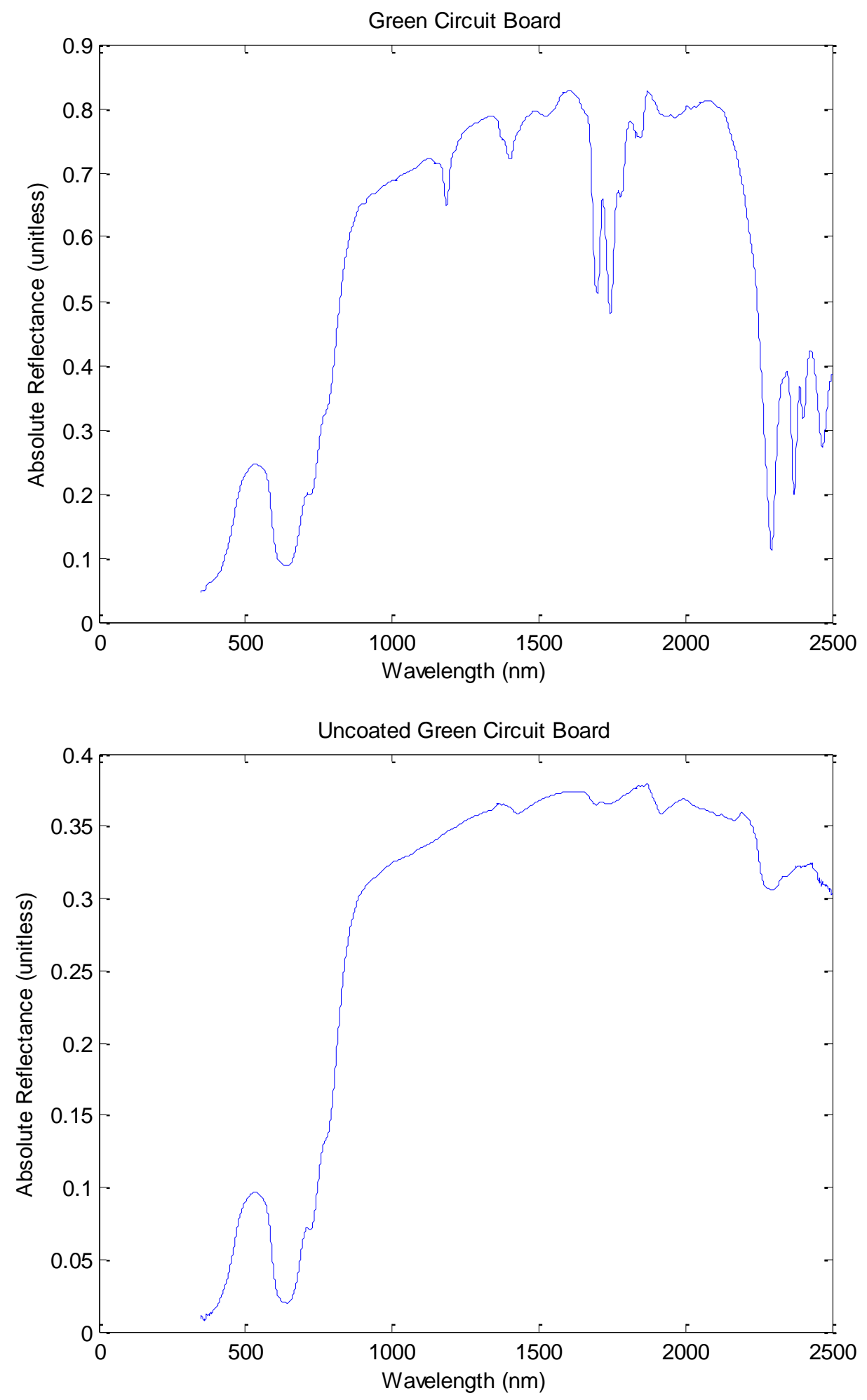
A p p endix A |A12
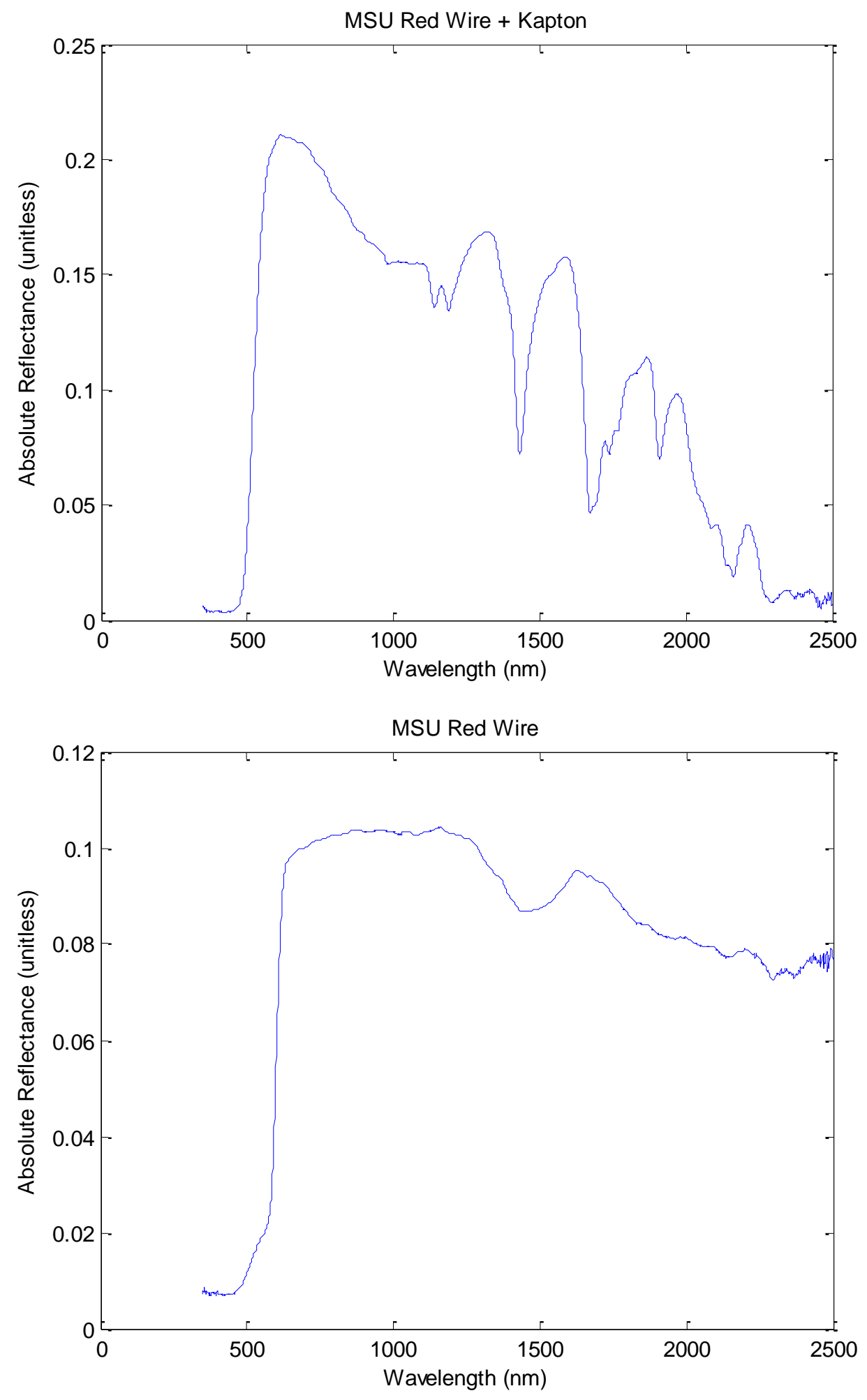
A p p endix A $\mid$ A13
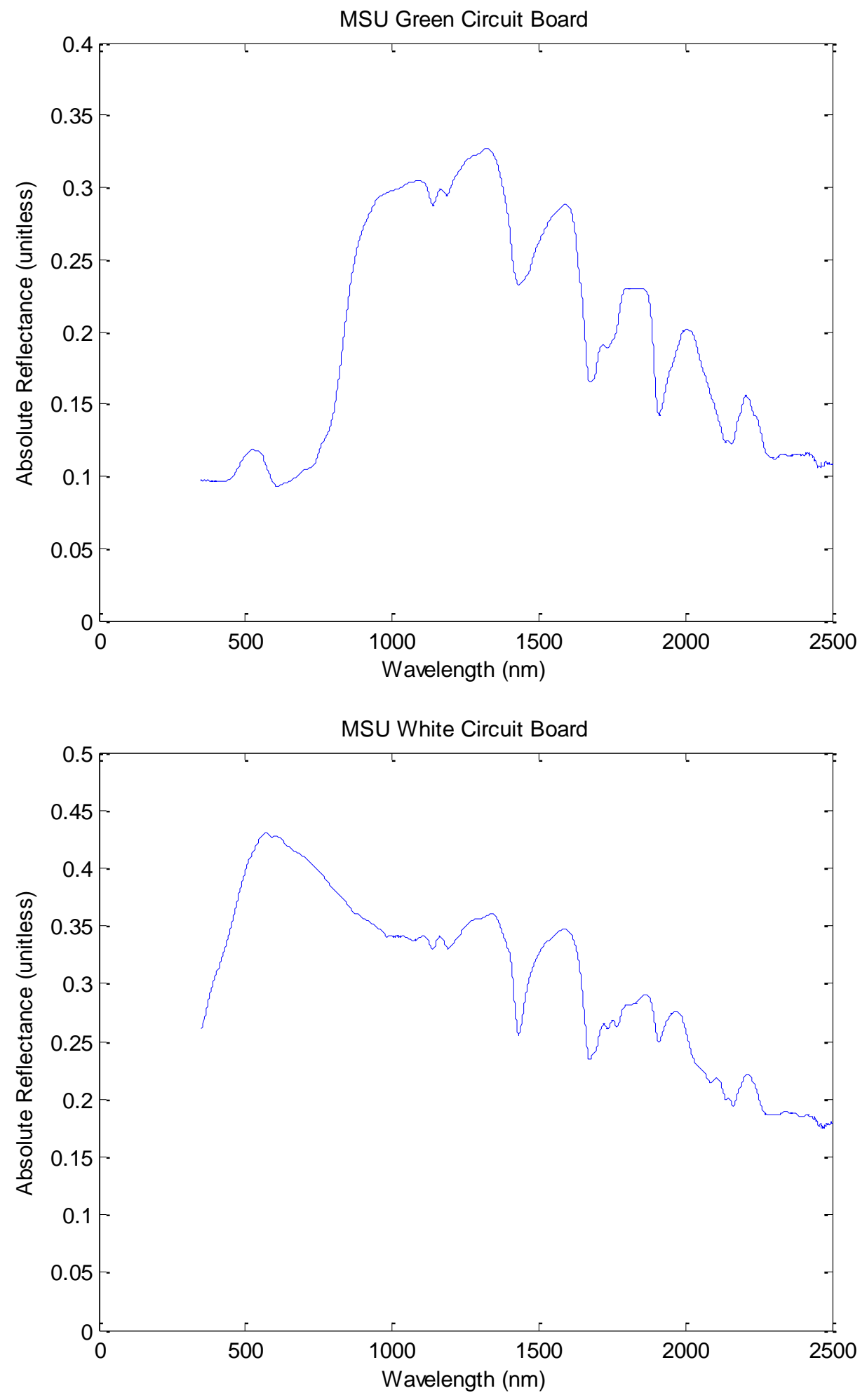
A p p endix A $\quad$ A14
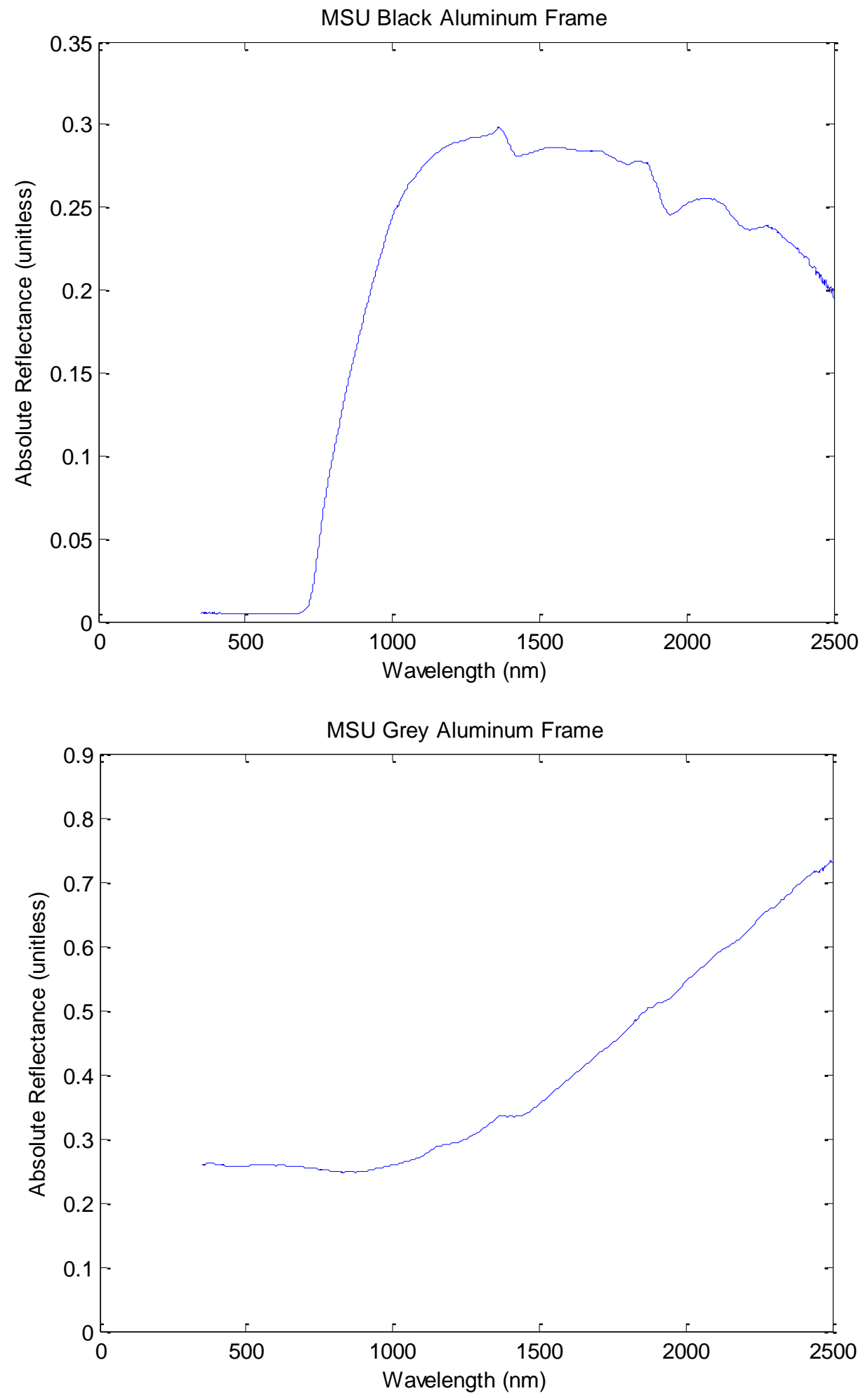
A p p endix A $\quad$ A15
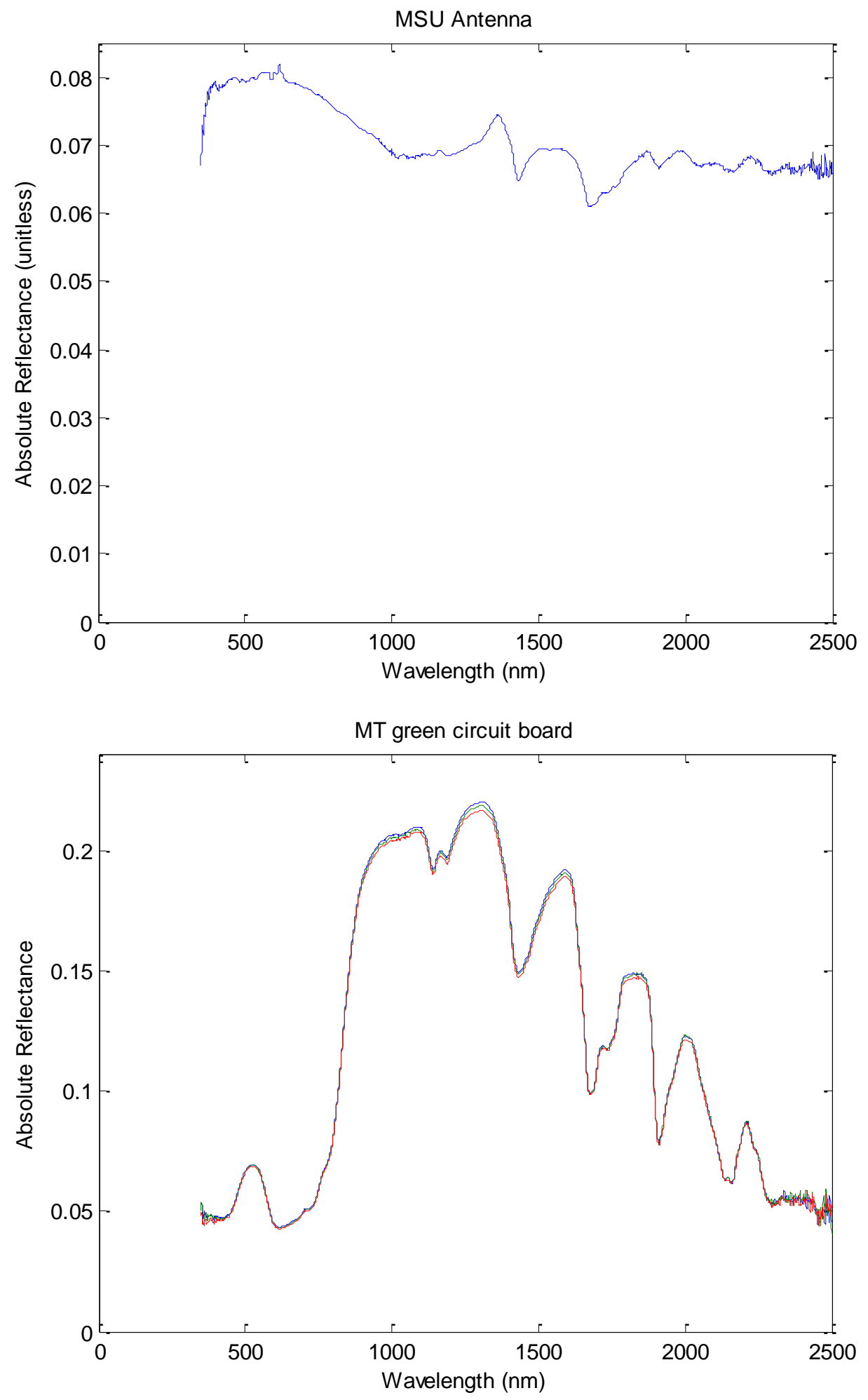
Appendix A |A16
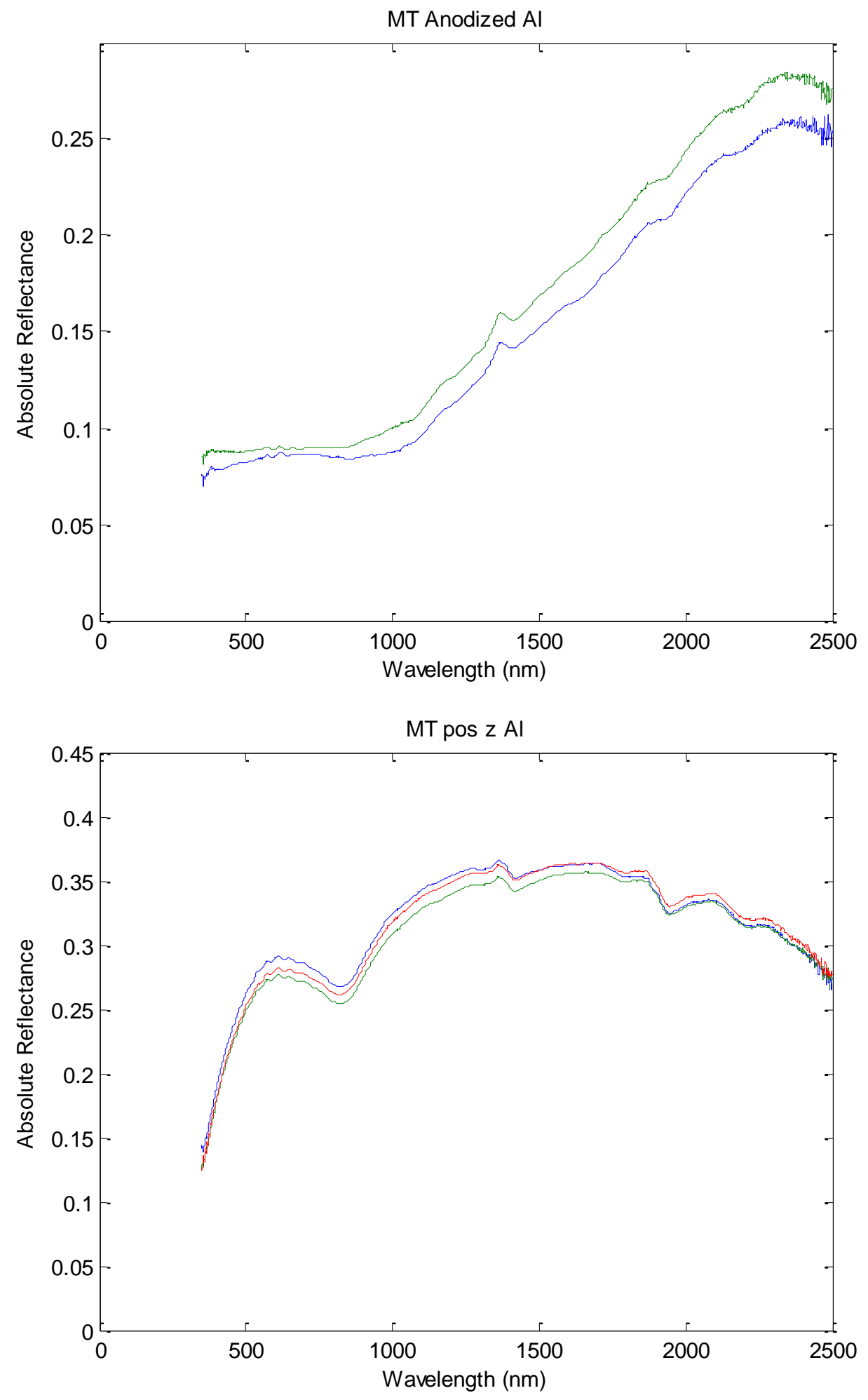
Appendix A |A17
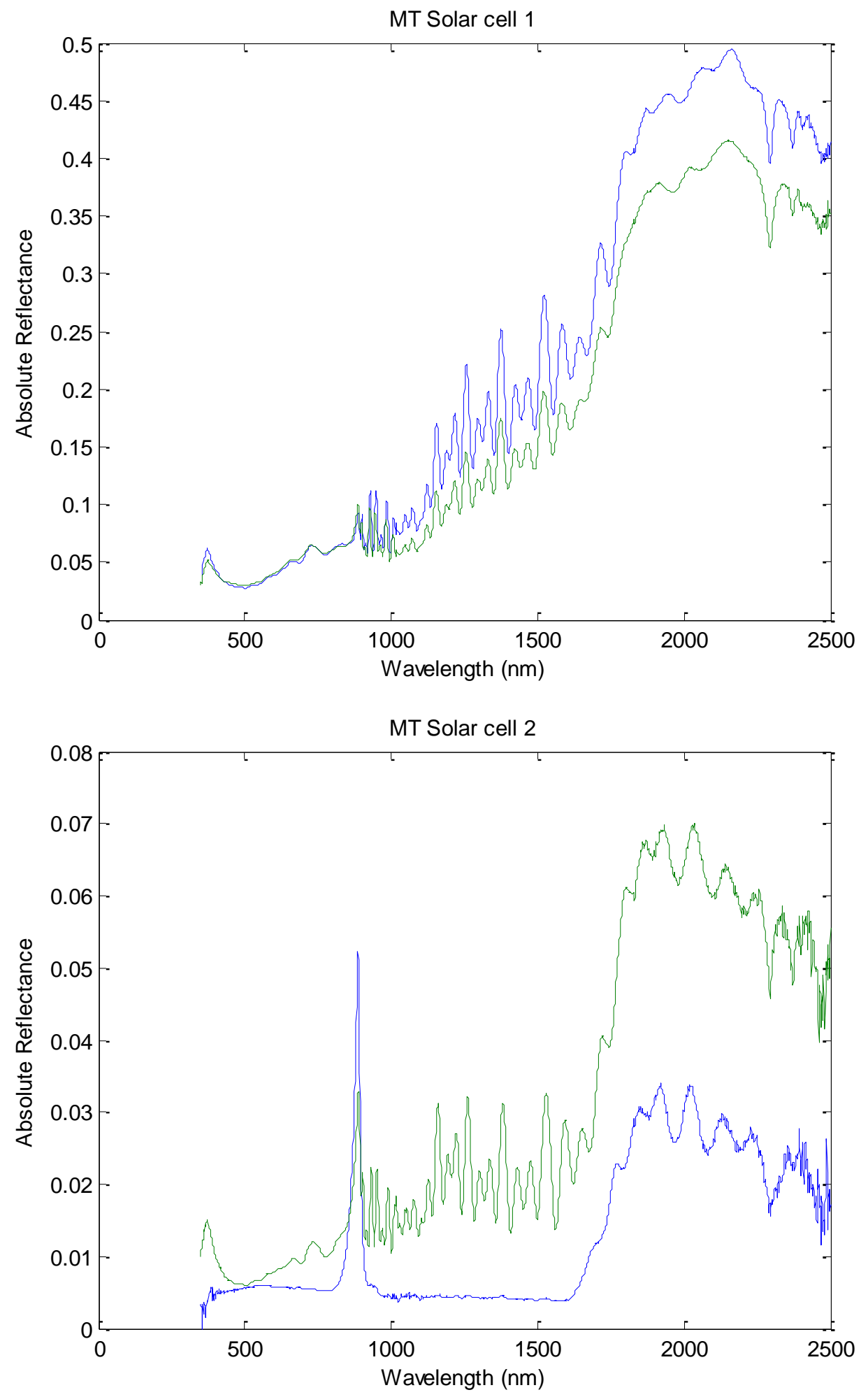
Appendix A |A18
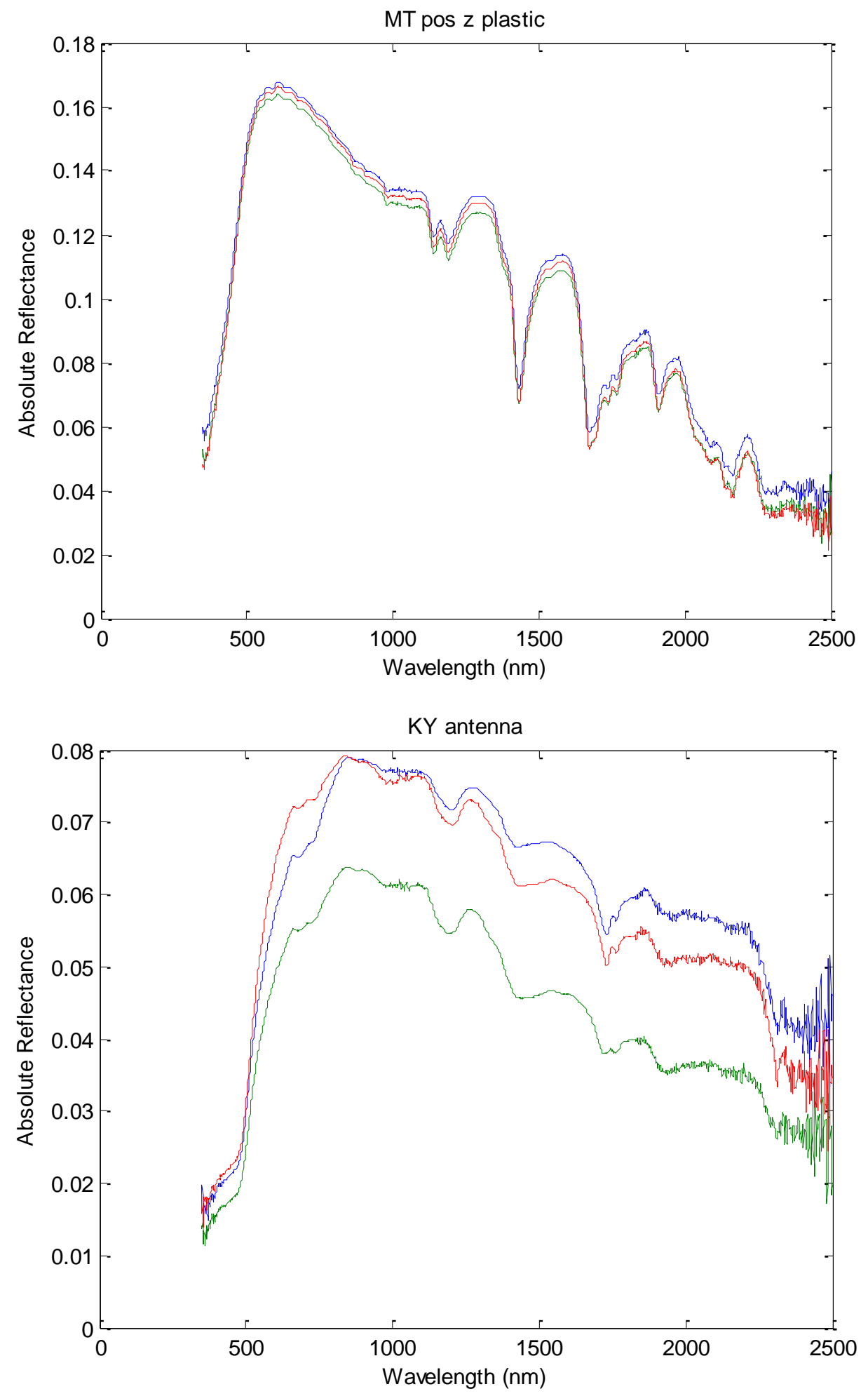
A p p endix A $\quad$ A19
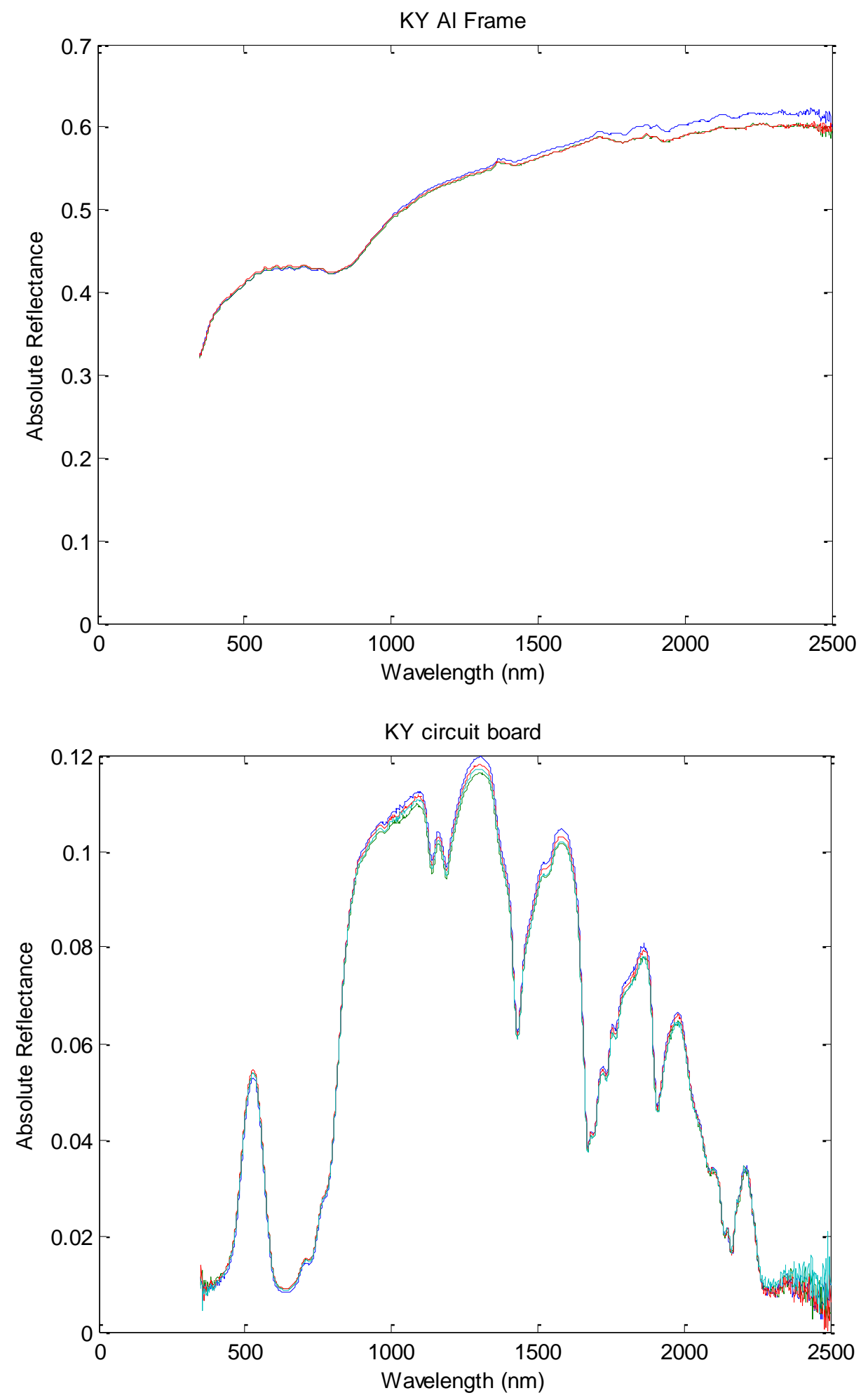
Appendix A |A20
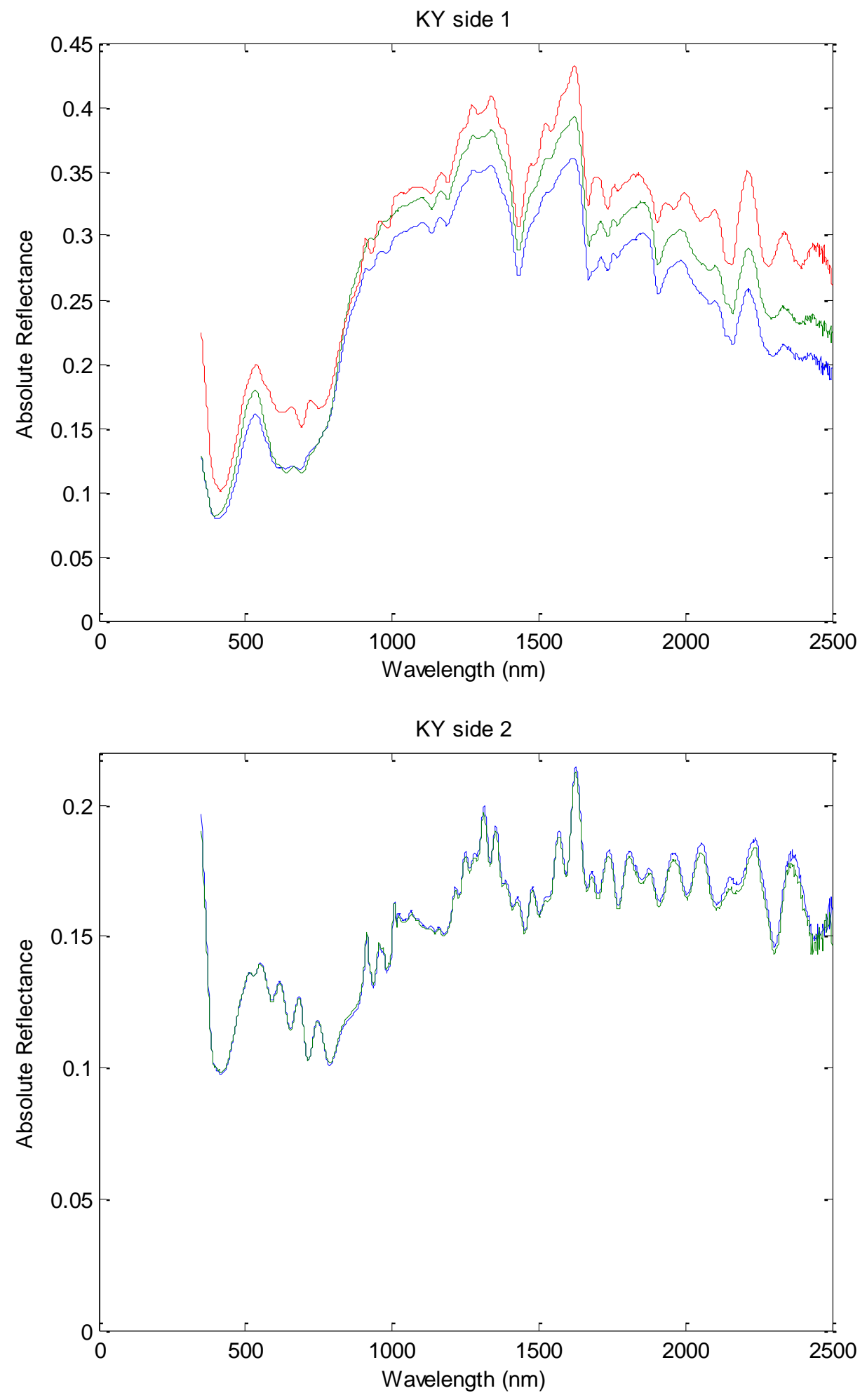
Appendix A |A21
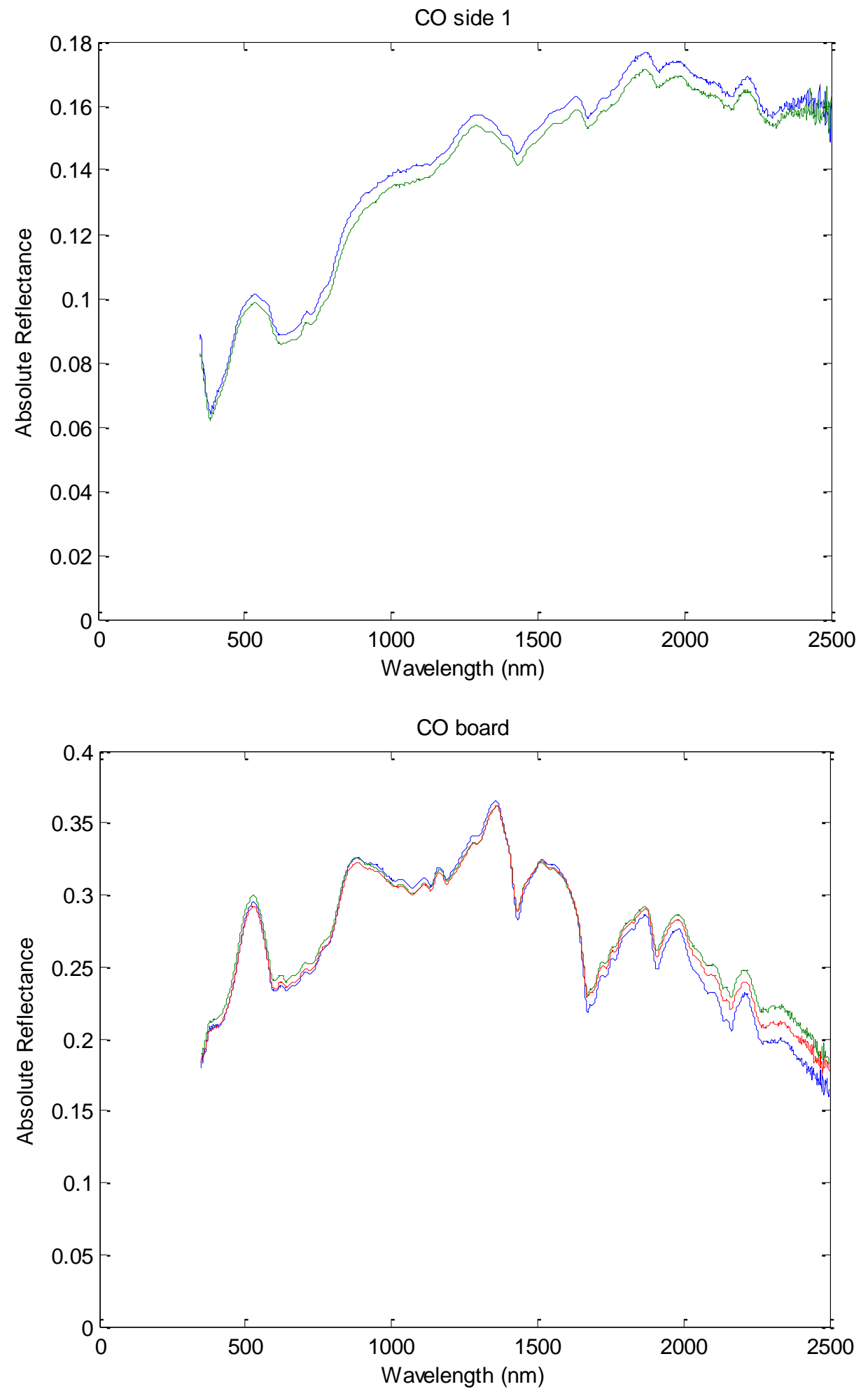
Appendix A $\mid$ A22
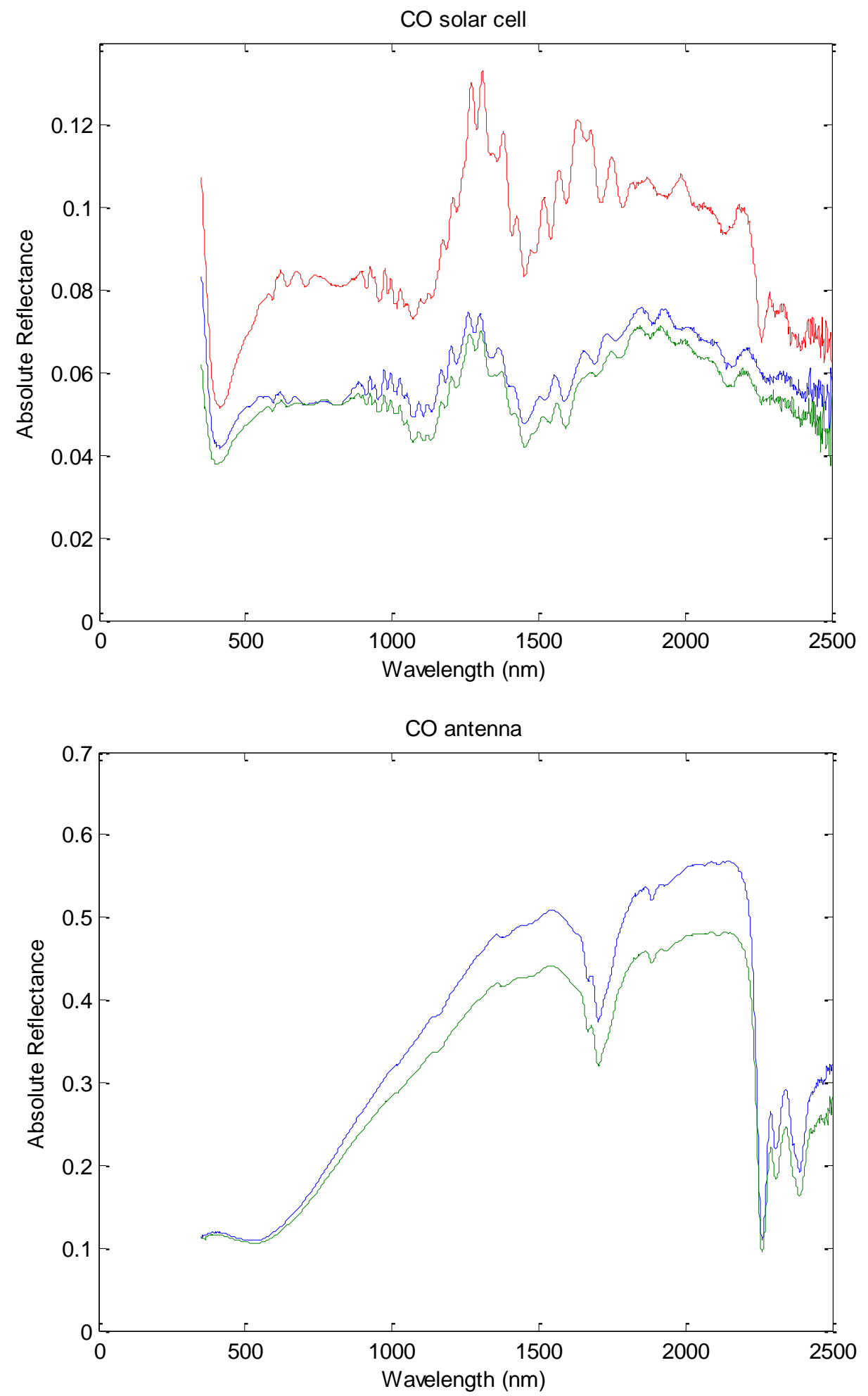
Appendix A |A23
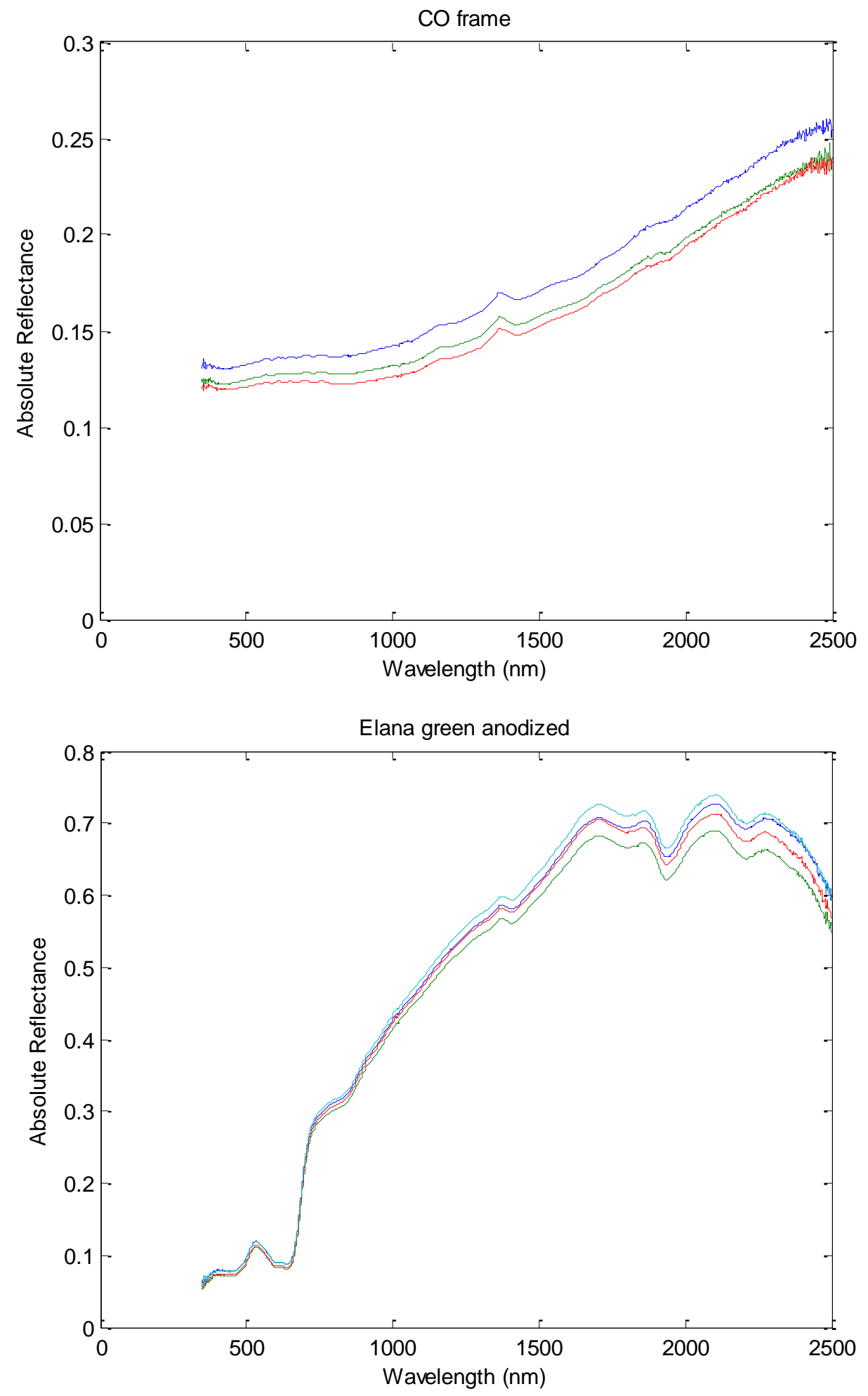
Appendix A |A24

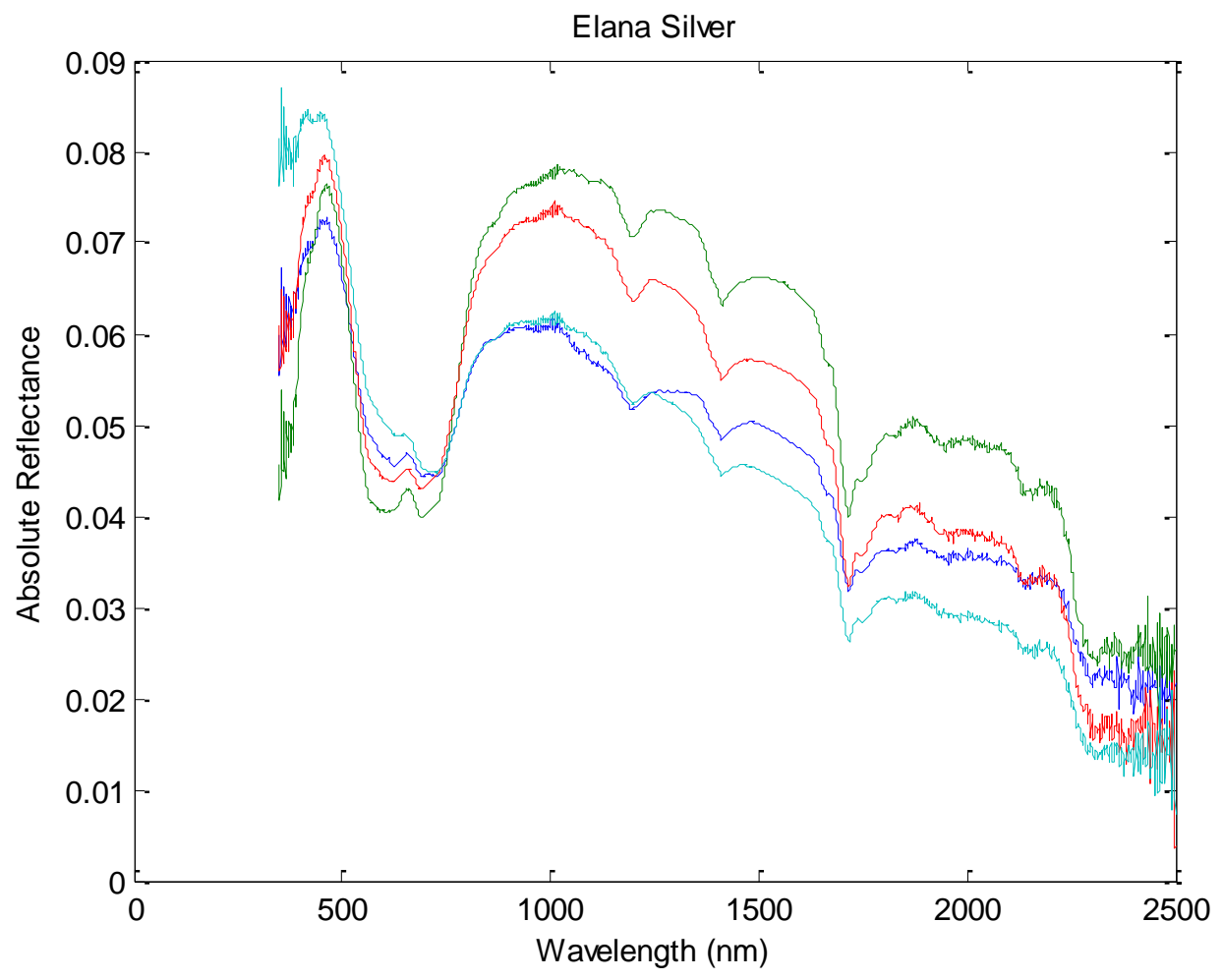




\section{Appendix B - All Unmixing Results}

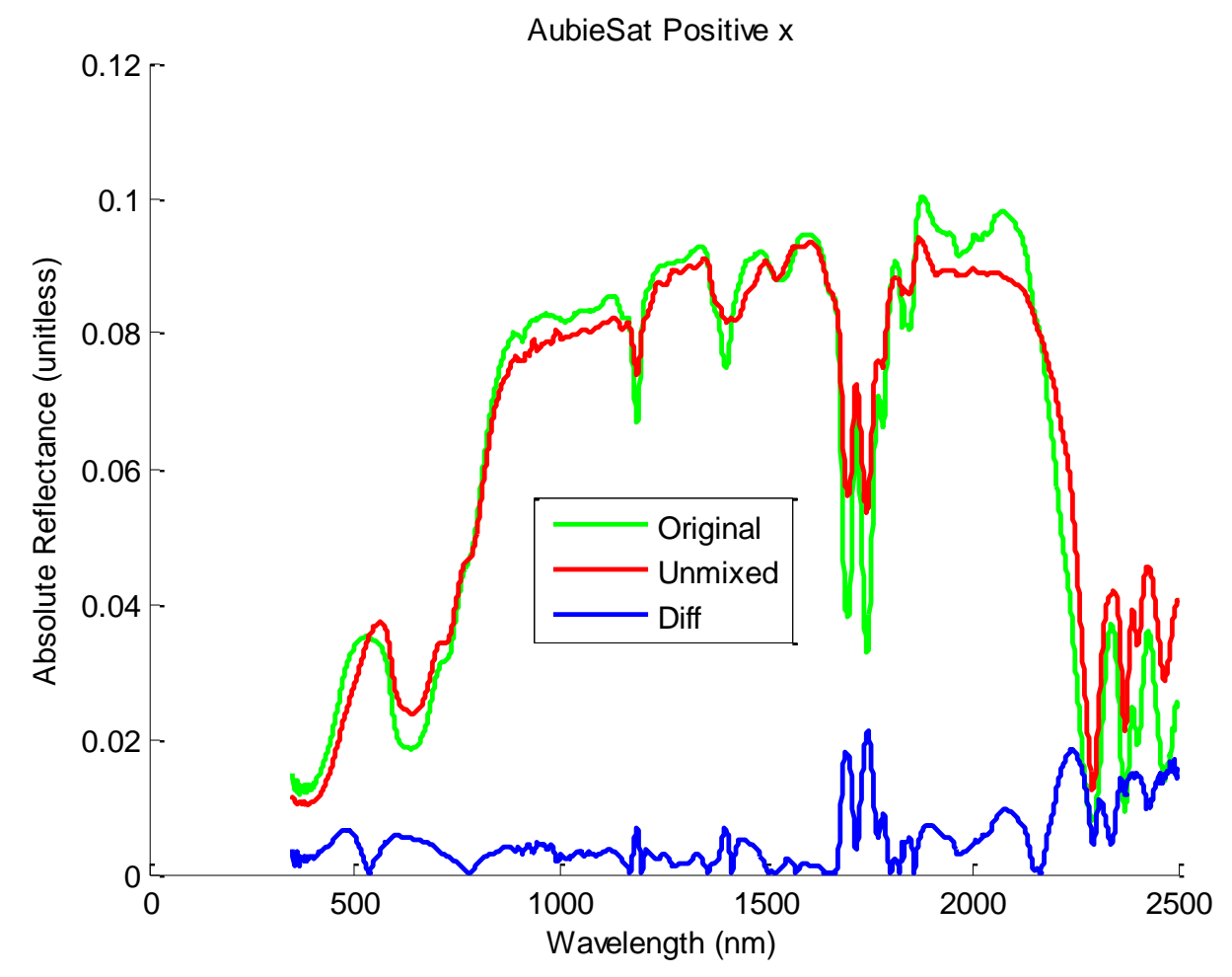

AubieSat Positive $x$

Unmixing results:

$\begin{array}{lll}0.000000 & \text { Solar Cell } 1 \quad \text { (AubieSat) } \\ 0.444035 & \text { Solar Cell 2 (AubieSat) } \\ 0.356651 & \text { Green Circuit Board (AubieSat) } \\ 0.000000 & \text { Uncoated Green Board (AubieSat) } \\ 0.000000 & \text { Solar Cell 3 (MSU Sat) } \\ 0.199314 & \text { Red Wire with Kapton (MSU Sat) } \\ 0.000000 & \text { Red Wire (MSU Sat) } \\ 0.000000 & \text { Green Circuit Board (MSU Sat) } \\ 0.000000 & \text { White Circuit Board (MSU Sat) } \\ 0.000000 & \text { Black Anodized Aluminum (MSU Sat) } \\ 0.000000 & \text { Grey Anodized Aluminum (MSU Sat) } \\ 0.000000 & \text { Antenna (MSU Sat) } \\ 0.000000 & \text { Solar Cell, no sinusoid feature } \\ 0.000000 & \text { Solar Cell, sinusoid feature min TF } \\ 0.000000 & \text { Solar Cell, sinusoid feature } \\ 0.000000 & \text { Solar Cell, sinusoid feature, alt } \\ 0.097480 & \text { Vector Norm Approximation Error }\end{array}$




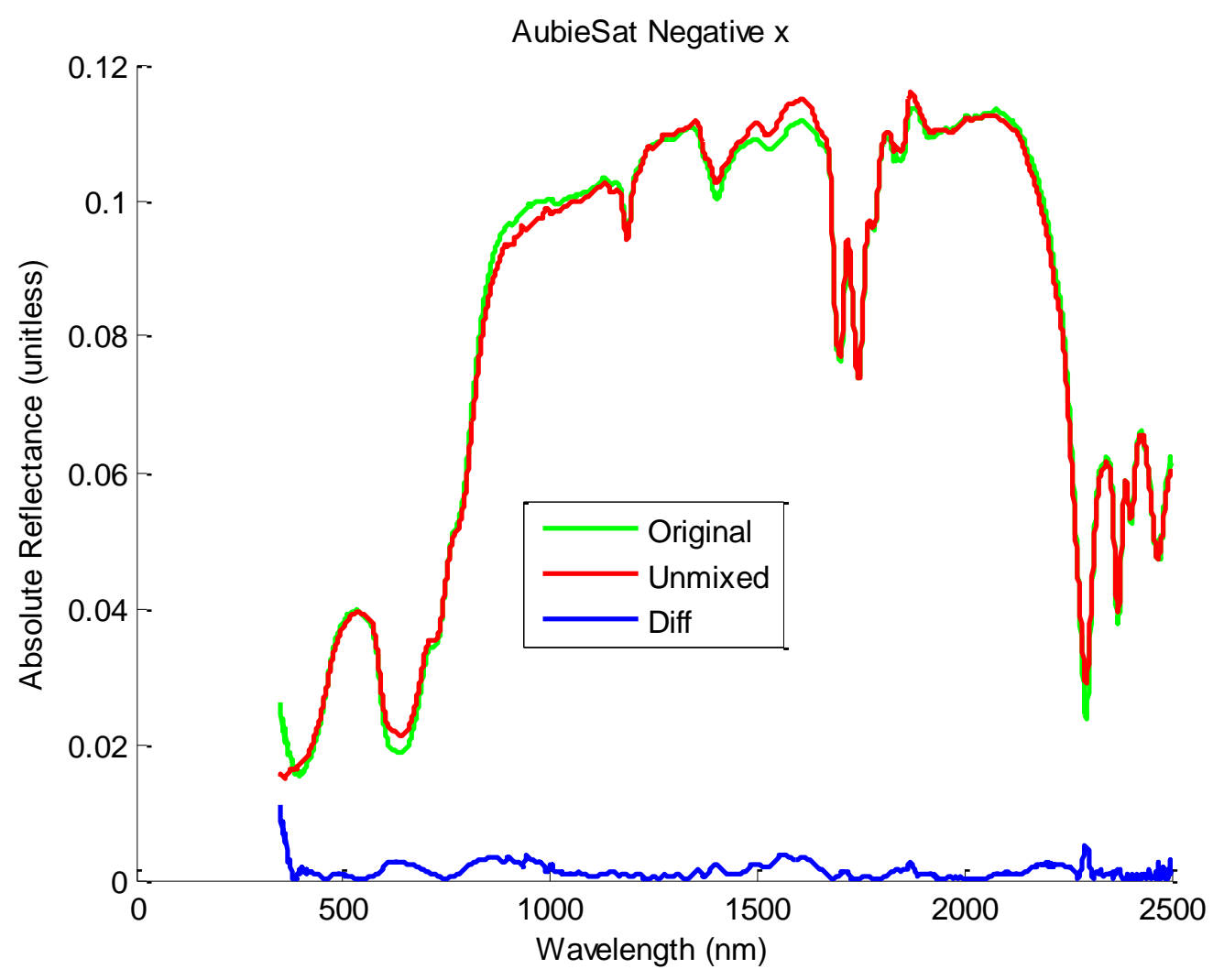

AubieSat Negative $x$

Unmixing results:

\begin{tabular}{|c|c|}
\hline 0.000000 & Solar Cell 1 \\
\hline 0.229884 & Solar Cell $2 \quad$ (AubieSat) \\
\hline 0.351670 & Green Circuit Board $\quad$ (AubieSat) \\
\hline 0.089279 & Uncoated Green Board (AubieSat) \\
\hline 0.000000 & Solar Cell $3 \quad$ (MSU Sat) \\
\hline 0.000000 & Red Wire with Kapton (MSU Sat) \\
\hline 0.071809 & Red Wire $\quad$ (MSU Sat) \\
\hline 0.041091 & Green Circuit Board (MSU Sat) \\
\hline 0.000000 & White Circuit Board \\
\hline 0.000000 & Black Anodized Aluminum (MSU Sat) \\
\hline 0.000000 & Grey Anodized Aluminum (MSU Sat) \\
\hline 0.216267 & Antenna $\quad$ (MSU Sat) \\
\hline 0.000000 & Solar Cell, no sinusoid feature \\
\hline 0.000000 & Solar Cell, sinusoid feature min TF \\
\hline 0.000000 & Solar Cell, sinusoid feature \\
\hline 0.000000 & Solar Cell, sinusoid feature, alt \\
\hline
\end{tabular}


0.019725 Vector Norm Approximation Error

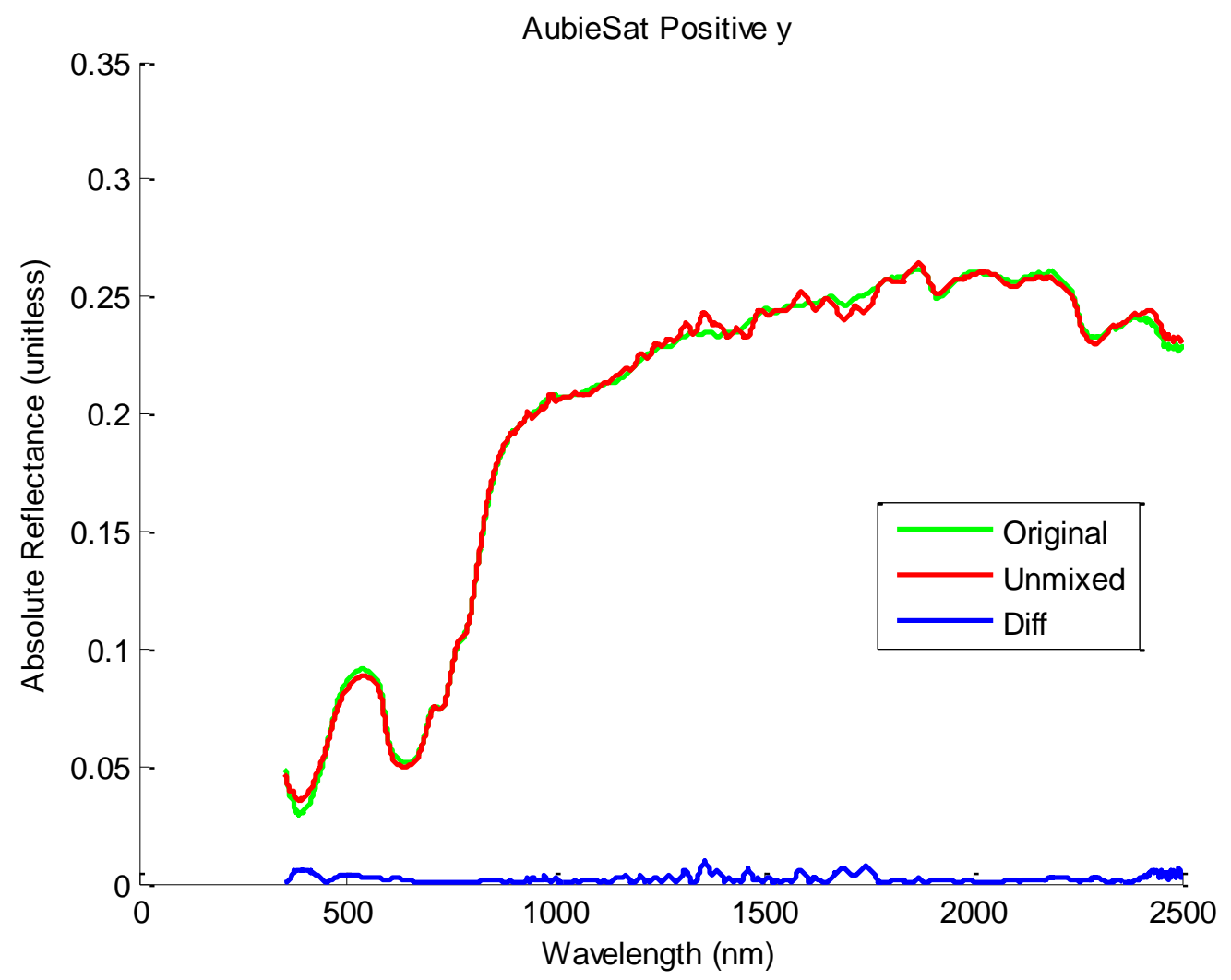

AubieSat Positive y

Unmixing results:

\begin{tabular}{|c|c|}
\hline 0.211560 & Solar Cell 1 \\
\hline 0.092042 & Solar Cell 2 \\
\hline 0.000000 & Green Circuit Board (AubieSat) \\
\hline 0.571268 & Uncoated Green Board (AubieSat) \\
\hline 0.000000 & Solar Cell $3 \quad$ (MSU Sat) \\
\hline 0.027451 & Red Wire with Kapton $\quad$ (MSU Sat) \\
\hline 0.000000 & Red Wire $\quad$ (MSU Sat) \\
\hline 0.000000 & Green Circuit Board (MSU Sat) \\
\hline 0.016367 & White Circuit Board \\
\hline 0.000000 & Black Anodized Aluminum (MSU Sat) \\
\hline 0.073708 & Grey Anodized Aluminum (MSU Sat) \\
\hline 0.000000 & Antenna $\quad$ (MSU Sat) \\
\hline 0.000000 & Solar Cell, no sinusoid feature \\
\hline 0.000000 & Solar Cell, sinusoid feature min TF \\
\hline 0.006975 & Solar Cell, sinusoid feature \\
\hline 0.000628 & Solar Cell, sinusoid feature, alt \\
\hline 0.012298 & Vector Norm Approximation Error \\
\hline
\end{tabular}




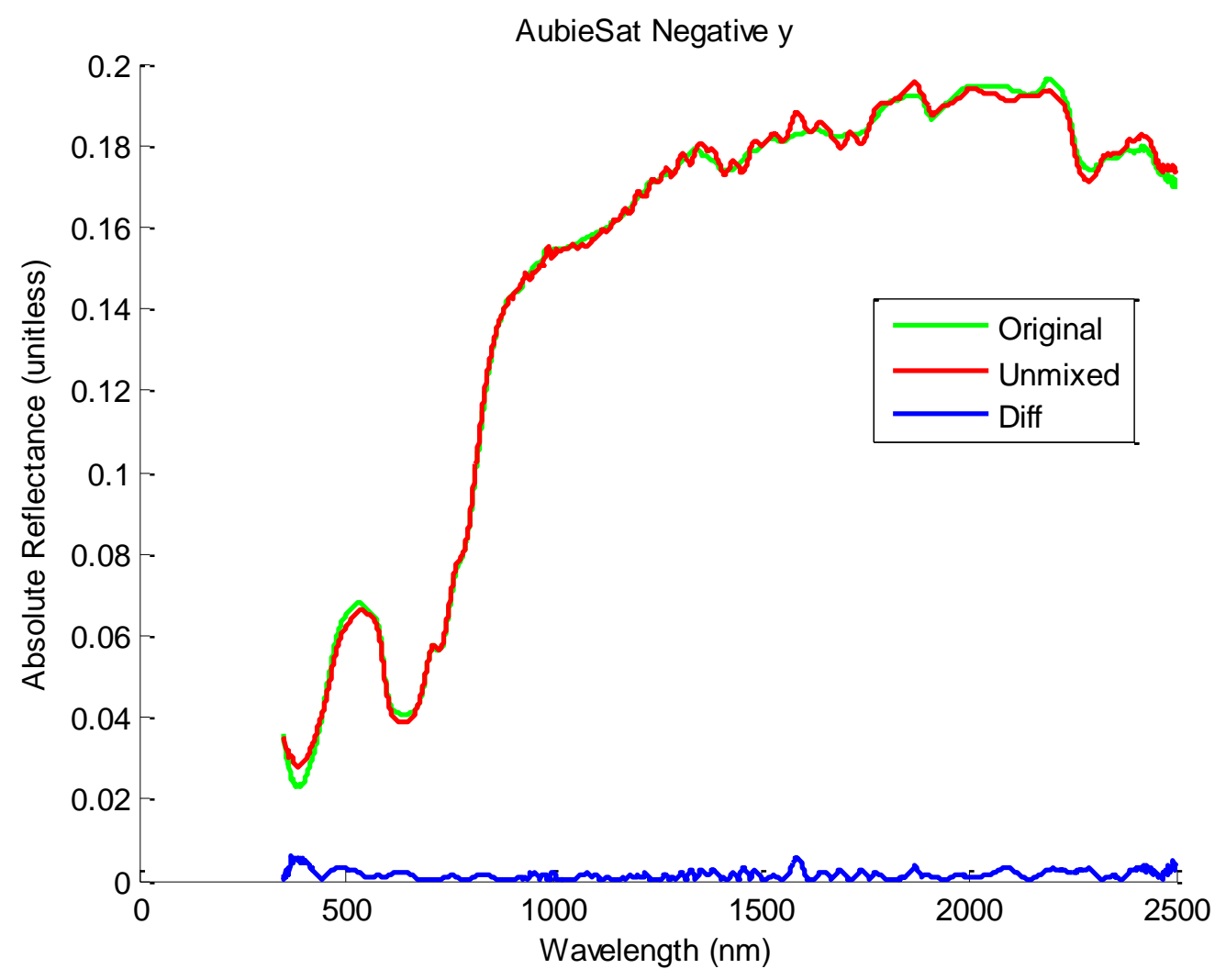

AubieSat Negative y

Unmixing results:

\begin{tabular}{|c|c|}
\hline 0.227786 & Solar Cell 1 \\
\hline 0.000311 & Solar Cell $2 \quad$ (AubieSat) \\
\hline 0.011414 & Green Circuit Board (AubieSat) \\
\hline 0.586222 & Uncoated Green Board (AubieSat) \\
\hline 0.010887 & Solar Cell $3 \quad$ (MSU Sat) \\
\hline 0.016697 & Red Wire with Kapton $\quad$ (MSU Sat) \\
\hline 0.027313 & Red Wire $\quad$ (MSU Sat) \\
\hline 0.000000 & Green Circuit Board (MSU Sat) \\
\hline 0.026463 & White Circuit Board \\
\hline 0.000000 & Black Anodized Aluminum (MSU Sat) \\
\hline 0.085711 & Grey Anodized Aluminum (MSU Sat) \\
\hline 0.000000 & Antenna $\quad$ (MSU Sat) \\
\hline 0.000000 & Solar Cell, no sinusoid feature \\
\hline 0.000000 & Solar Cell, sinusoid feature min TF \\
\hline 0.002355 & Solar Cell, sinusoid feature \\
\hline 0.004841 & Solar Cell, sinusoid feature, alt \\
\hline 0.011687 & Vector Norm Approximation Error \\
\hline
\end{tabular}




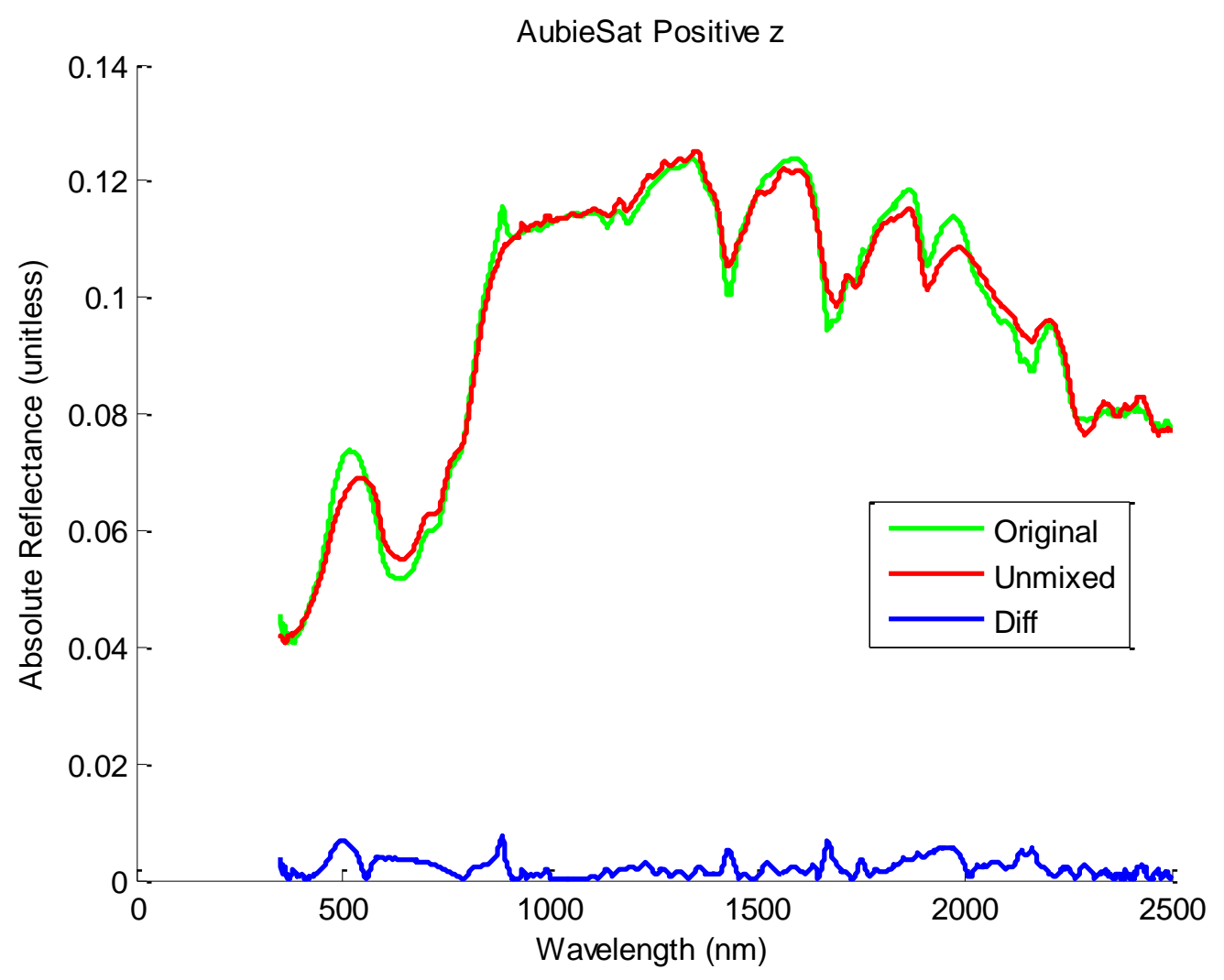

AubieSat Positive $z$

Unmixing results:

$\begin{array}{ll}0.000000 & \text { Solar Cell 1 } \quad \text { (AubieSat) } \\ 0.249012 & \text { Solar Cell 2 (AubieSat) } \\ 0.004751 & \text { Green Circuit Board (AubieSat) } \\ 0.338567 & \text { Uncoated Green Board (AubieSat) } \\ 0.000000 & \text { Solar Cell 3 (MSU Sat) } \\ 0.000000 & \text { Red Wire with Kapton (MSU Sat) } \\ 0.000000 & \text { Red Wire (MSU Sat) } \\ 0.191067 & \text { Green Circuit Board (MSU Sat) } \\ 0.211891 & \text { White Circuit Board (MSU Sat) } \\ 0.000000 & \text { Black Anodized Aluminum (MSU Sat) } \\ 0.000000 & \text { Grey Anodized Aluminum (MSU Sat) } \\ 0.000000 & \text { Antenna (MSU Sat) } \\ 0.000000 & \text { Solar Cell, no sinusoid feature } \\ 0.000000 & \text { Solar Cell, sinusoid feature min TF } \\ 0.004712 & \text { Solar Cell, sinusoid feature } \\ 0.000000 & \text { Solar Cell, sinusoid feature, alt } \\ & \end{array}$




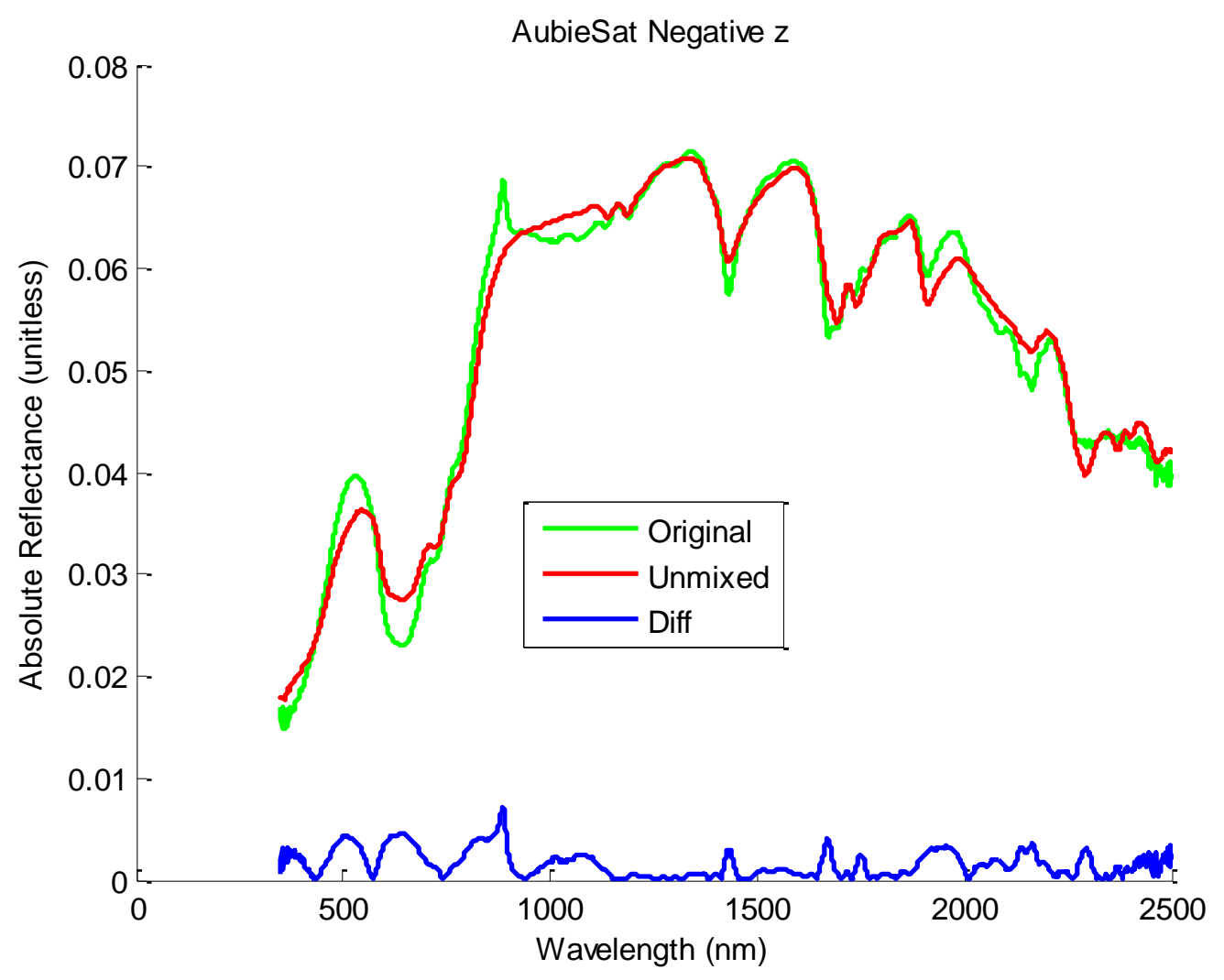

AubieSat Negative $z$

Unmixing results:

$\begin{array}{ll}0.000000 & \text { Solar Cell 1 } \quad \text { (AubieSat) } \\ 0.000000 & \text { Solar Cell 2 (AubieSat) } \\ 0.059187 & \text { Green Circuit Board (AubieSat) } \\ 0.420923 & \text { Uncoated Green Board (AubieSat) } \\ 0.000000 & \text { Solar Cell 3 (MSU Sat) } \\ 0.037061 & \text { Red Wire with Kapton (MSU Sat) } \\ 0.000000 & \text { Red Wire (MSU Sat) } \\ 0.247936 & \text { Green Circuit Board (MSU Sat) } \\ 0.234893 & \text { White Circuit Board (MSU Sat) } \\ 0.000000 & \text { Black Anodized Aluminum (MSU Sat) } \\ 0.000000 & \text { Grey Anodized Aluminum (MSU Sat) } \\ 0.000000 & \text { Antenna (MSU Sat) } \\ 0.000000 & \text { Solar Cell, no sinusoid feature } \\ 0.000000 & \text { Solar Cell, sinusoid feature min TF } \\ 0.000000 & \text { Solar Cell, sinusoid feature } \\ 0.000000 & \text { Solar Cell, sinusoid feature, alt } \\ 0.037202 & \text { Vector Norm Approximation Error }\end{array}$




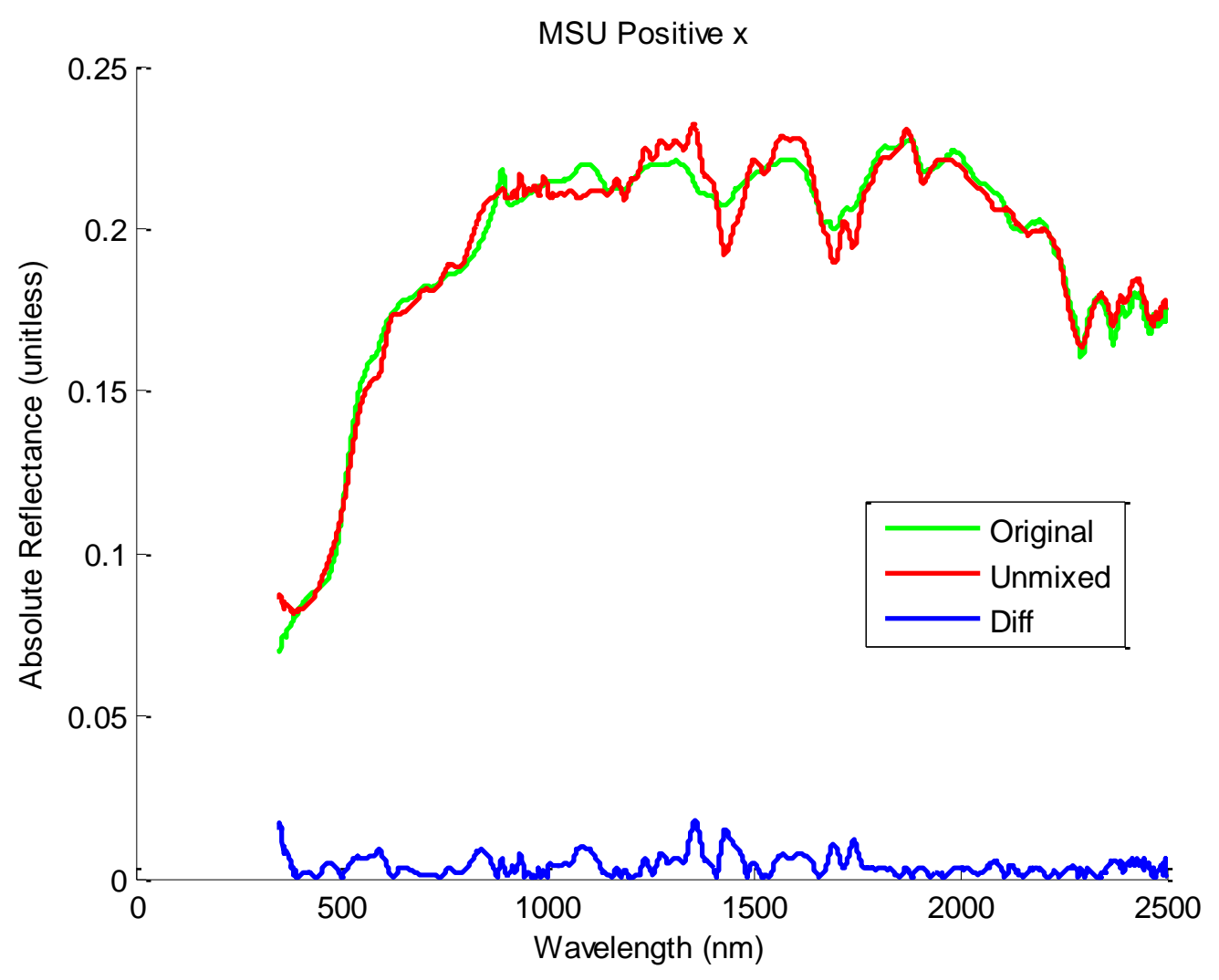

MSU Positive $x$

Unmixing results:

\begin{tabular}{|c|c|}
\hline 0.032070 & Solar Cell 1 \\
\hline 0.238752 & Solar Cell $2 \quad$ (AubieSat) \\
\hline 0.004003 & Green Circuit Board ～(AubieSat) \\
\hline 0.092629 & Uncoated Green Board (AubieSat) \\
\hline 0.000000 & Solar Cell $3 \quad$ (MSU Sat) \\
\hline 0.123123 & Red Wire with Kapton (MSU Sat) \\
\hline 0.243135 & Red Wire $\quad$ (MSU Sat) \\
\hline 0.000000 & Green Circuit Board (MSU Sat) \\
\hline 0.040052 & White Circuit Board \\
\hline 0.000000 & Black Anodized Aluminum (MSU Sat) \\
\hline 0.034555 & Grey Anodized Aluminum (MSU Sat) \\
\hline 0.191681 & Antenna $\quad$ (MSU Sat) \\
\hline 0.000000 & Solar Cell, no sinusoid feature \\
\hline 0.000000 & Solar Cell, sinusoid feature min TF \\
\hline 0.000000 & Solar Cell, sinusoid feature \\
\hline 0.000000 & Solar Cell, sinusoid feature, alt \\
\hline 0.025898 & Vector Norm Approximation Error \\
\hline
\end{tabular}




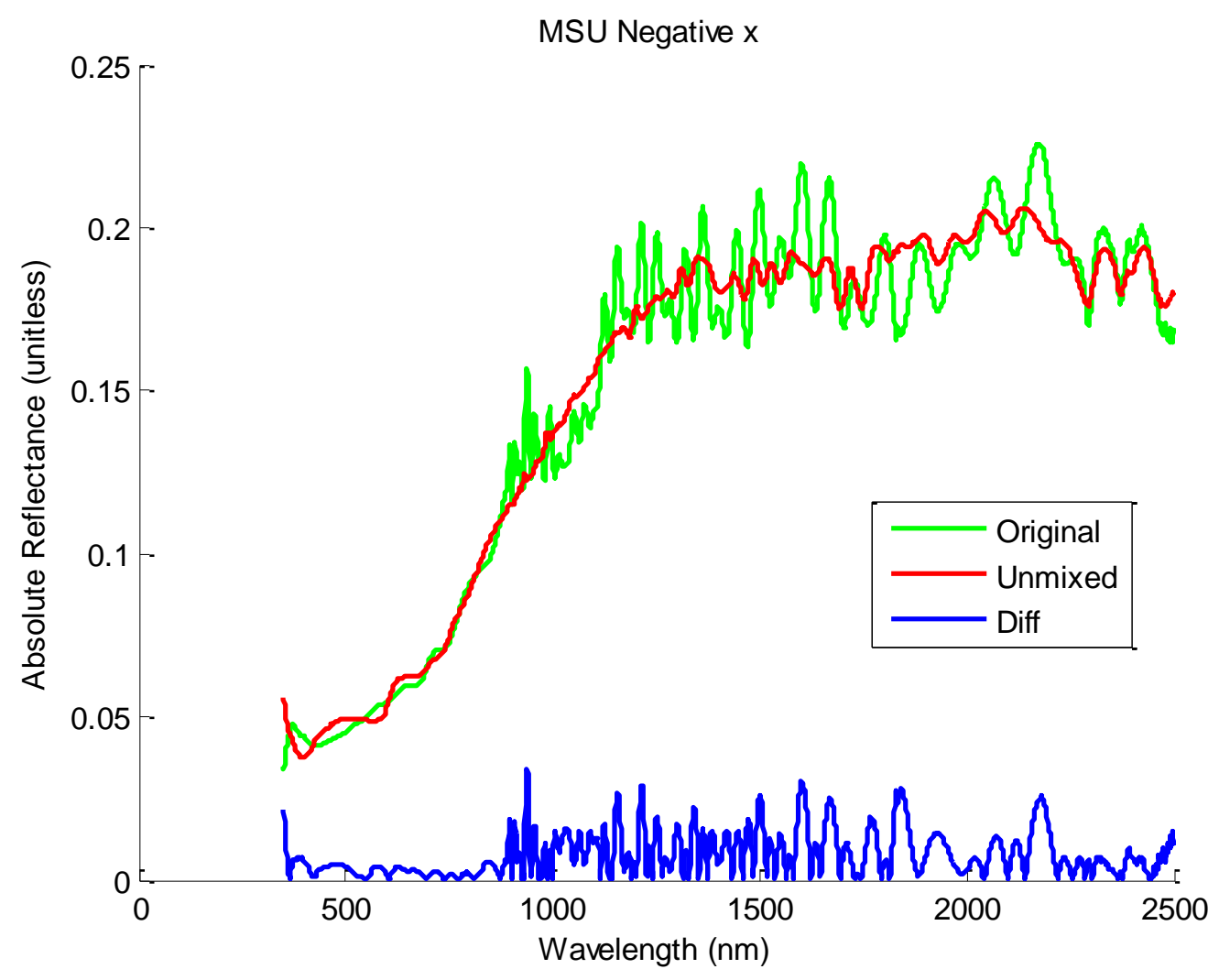

MSU Negative $x$

Unmixing results:

$\begin{array}{lll}0.230177 & \text { Solar Cell } 1 \quad \text { (AubieSat) } \\ 0.000000 & \text { Solar Cell 2 (AubieSat) } \\ 0.043239 & \text { Green Circuit Board (AubieSat) } \\ 0.022424 & \text { Uncoated Green Board (AubieSat) } \\ 0.000000 & \text { Solar Cell 3 (MSU Sat) } \\ 0.000000 & \text { Red Wire with Kapton (MSU Sat) } \\ 0.332436 & \text { Red Wire (MSU Sat) } \\ 0.000000 & \text { Green Circuit Board (MSU Sat) } \\ 0.000000 & \text { White Circuit Board (MSU Sat) } \\ 0.172777 & \text { Black Anodized Aluminum (MSU Sat) } \\ 0.071798 & \text { Grey Anodized Aluminum (MSU Sat) } \\ 0.000000 & \text { Antenna (MSU Sat) } \\ 0.072171 & \text { Solar Cell, no sinusoid feature } \\ 0.030570 & \text { Solar Cell, sinusoid feature min TF } \\ 0.024410 & \text { Solar Cell, sinusoid feature } \\ 0.000000 & \text { Solar Cell, sinusoid feature, alt } \\ 0.064488 & \text { Vector Norm Approximation Error }\end{array}$




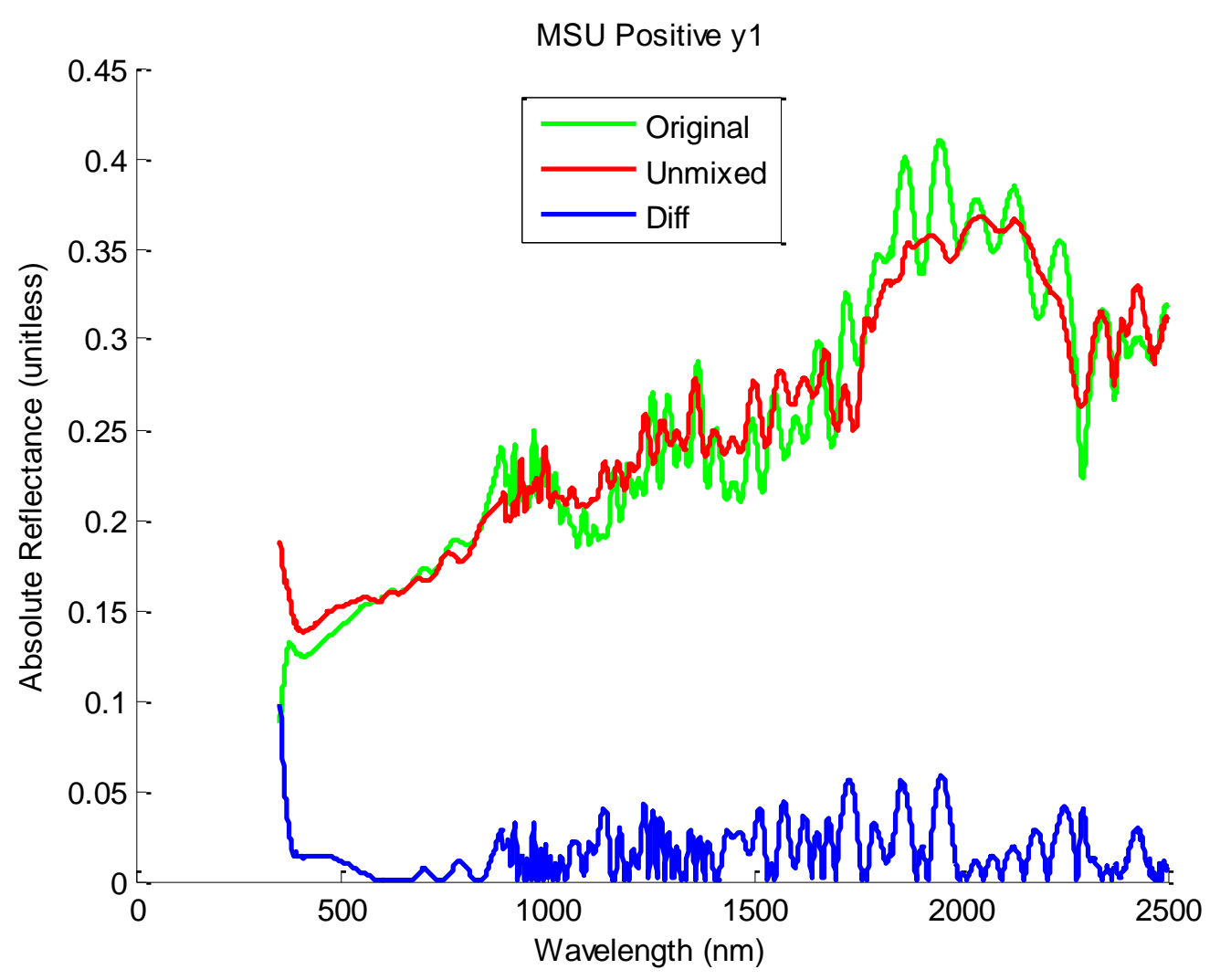

MSU Positive y1

Unmixing results:

$\begin{array}{ll}0.019760 & \text { Solar Cell 1 } \quad \text { (AubieSat) } \\ 0.767096 & \text { Solar Cell 2 (AubieSat) } \\ 0.000000 & \text { Green Circuit Board (AubieSat) } \\ 0.051340 & \text { Uncoated Green Board (AubieSat) } \\ 0.000000 & \text { Solar Cell 3 (MSU Sat) } \\ 0.000000 & \text { Red Wire with Kapton (MSU Sat) } \\ 0.057495 & \text { Red Wire (MSU Sat) } \\ 0.000000 & \text { Green Circuit Board (MSU Sat) } \\ 0.000000 & \text { White Circuit Board (MSU Sat) } \\ 0.000000 & \text { Black Anodized Aluminum (MSU Sat) } \\ 0.100577 & \text { Grey Anodized Aluminum (MSU Sat) } \\ 0.000000 & \text { Antenna (MSU Sat) } \\ 0.000000 & \text { Solar Cell, no sinusoid feature } \\ 0.000000 & \text { Solar Cell, sinusoid feature min TF } \\ 0.000000 & \text { Solar Cell, sinusoid feature } \\ 0.003731 & \text { Solar Cell, sinusoid feature, alt } \\ & \end{array}$




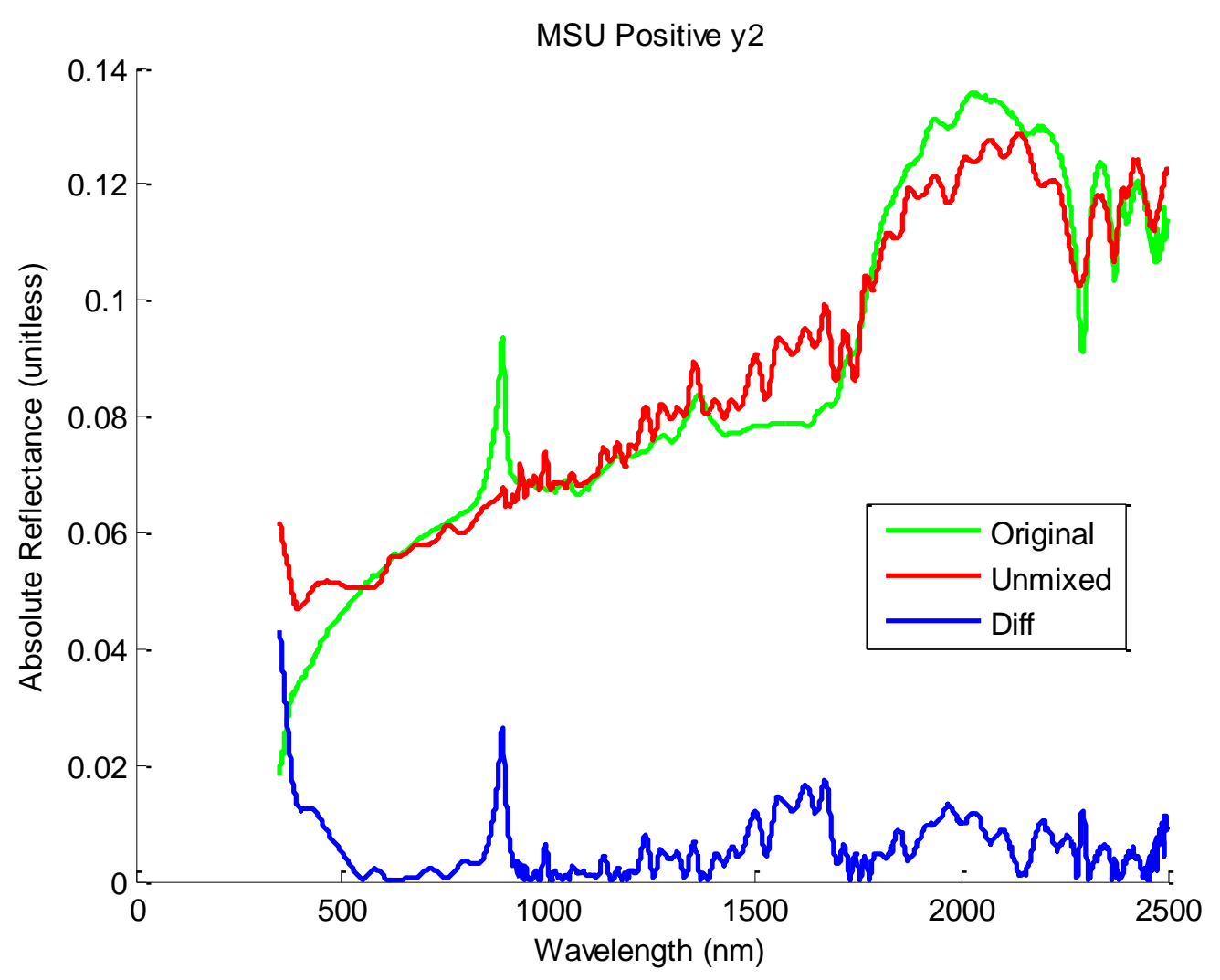

MSU Positive y2

Unmixing results:

\begin{tabular}{|c|c|}
\hline 0.000000 & Solar Cell 1 \\
\hline 0.641625 & Solar Cell 2 \\
\hline 0.019117 & Green Circuit Board $\quad$ (AubieSat) \\
\hline 0.000000 & Uncoated Green Board (AubieSat) \\
\hline 0.000000 & Solar Cell $3 \quad$ (MSU Sat) \\
\hline 0.000000 & Red Wire with Kapton (MSU Sat) \\
\hline 0.141062 & Red Wire $\quad$ (MSU Sat) \\
\hline 0.000000 & Green Circuit Board (MSU Sat) \\
\hline 0.000000 & White Circuit Board \\
\hline 0.000000 & Black Anodized Aluminum (MSU Sat) \\
\hline 0.171143 & Grey Anodized Aluminum (MSU Sat) \\
\hline 0.000000 & Antenna $\quad$ (MSU Sat) \\
\hline 0.000000 & Solar Cell, no sinusoid feature \\
\hline 0.000000 & Solar Cell, sinusoid feature min TF \\
\hline 0.000000 & Solar Cell, sinusoid feature \\
\hline 0.027052 & Solar Cell, sinusoid feature, alt \\
\hline 0.087724 & Vector Norm Approximation Error \\
\hline
\end{tabular}




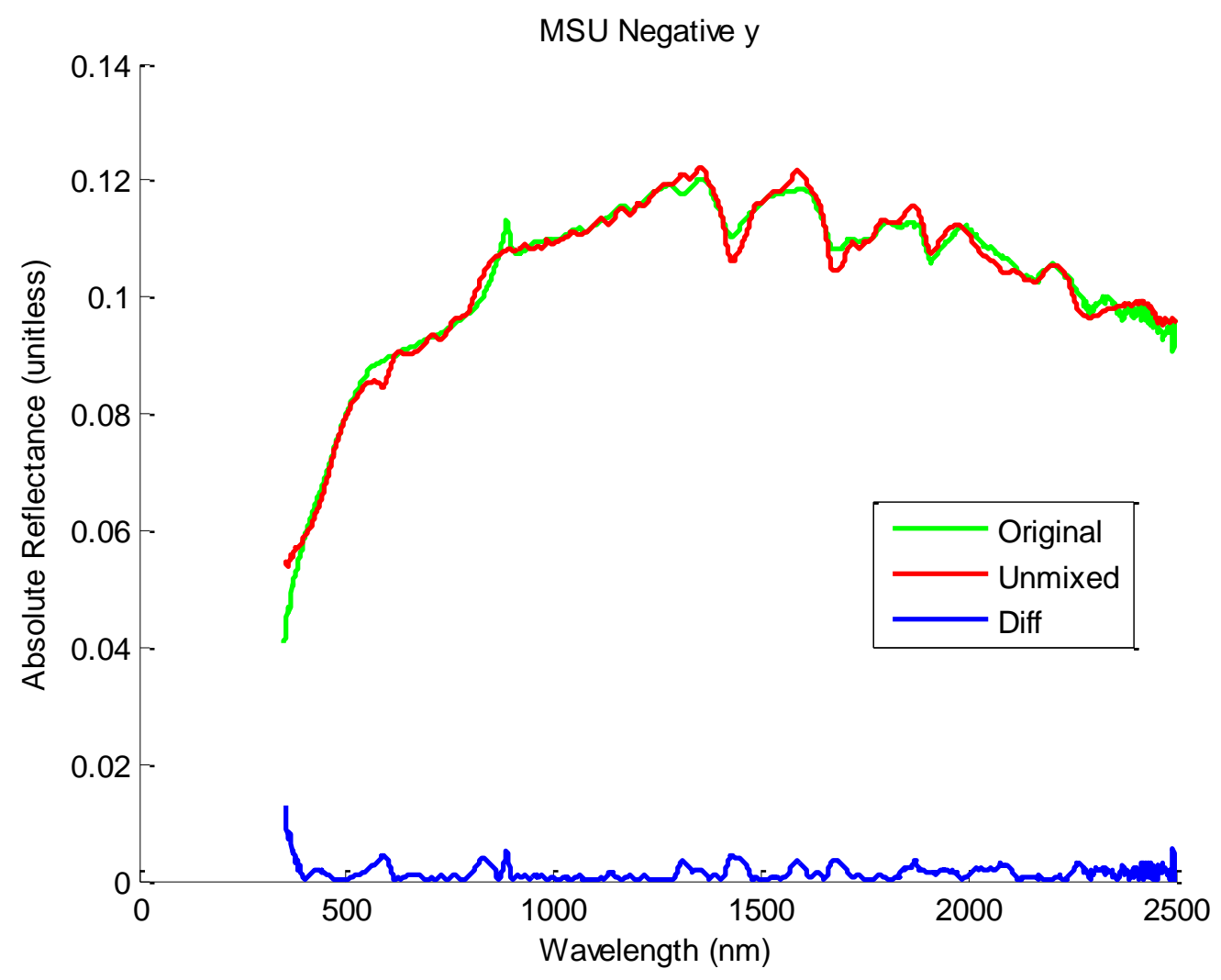

MSU Negative y

Unmixing results:

$\begin{array}{ll}0.089148 & \text { Solar Cell 1 } \quad \text { (AubieSat) } \\ 0.000000 & \text { Solar Cell 2 (AubieSat) } \\ 0.000000 & \text { Green Circuit Board (AubieSat) } \\ 0.154167 & \text { Uncoated Green Board (AubieSat) } \\ 0.000000 & \text { Solar Cell 3 (MSU Sat) } \\ 0.000000 & \text { Red Wire with Kapton (MSU Sat) } \\ 0.281955 & \text { Red Wire (MSU Sat) } \\ 0.000000 & \text { Green Circuit Board (MSU Sat) } \\ 0.217422 & \text { White Circuit Board (MSU Sat) } \\ 0.000000 & \text { Black Anodized Aluminum (MSU Sat) } \\ 0.030811 & \text { Grey Anodized Aluminum (MSU Sat) } \\ 0.207145 & \text { Antenna (MSU Sat) } \\ 0.019351 & \text { Solar Cell, no sinusoid feature } \\ 0.000000 & \text { Solar Cell, sinusoid feature min TF } \\ 0.000000 & \text { Solar Cell, sinusoid feature } \\ 0.000000 & \text { Solar Cell, sinusoid feature, alt } \\ 0.018076 & \text { Vector Norm Approximation Error }\end{array}$




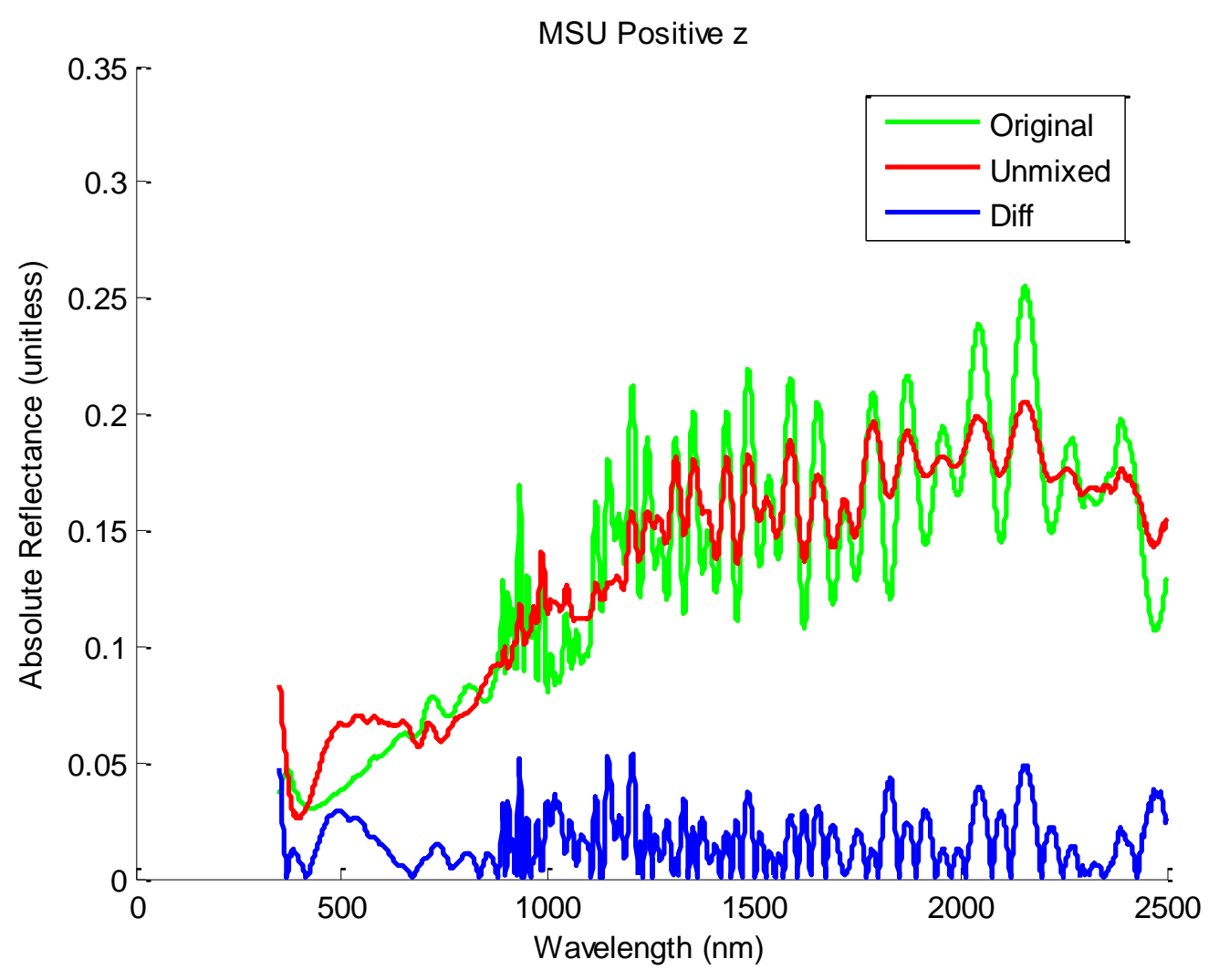

MSU Positive $z$

Unmixing results:

\begin{tabular}{|c|c|}
\hline 0.834242 & Solar Cell 1 \\
\hline 0.000000 & Solar Cell 2 \\
\hline 0.030976 & Green Circuit Board (AubieSat) \\
\hline 0.000000 & Uncoated Green Board (AubieSat) \\
\hline 0.000000 & Solar Cell $3 \quad$ (MSU Sat) \\
\hline 0.000000 & Red Wire with Kapton (MSU Sat) \\
\hline 0.094748 & Red Wire $\quad$ (MSU Sat) \\
\hline 0.000000 & Green Circuit Board (MSU Sat) \\
\hline 0.000000 & White Circuit Board \\
\hline 0.038817 & Black Anodized Aluminum (MSU Sat) \\
\hline 0.000000 & Grey Anodized Aluminum (MSU Sat) \\
\hline 0.000000 & Antenna $\quad$ (MSU Sat) \\
\hline 0.001218 & Solar Cell, no sinusoid feature \\
\hline 0.000000 & Solar Cell, sinusoid feature min TF \\
\hline 0.000000 & Solar Cell, sinusoid feature \\
\hline 0.000000 & Solar Cell, sinusoid feature, alt \\
\hline 0.135314 & Vector Norm Approximation Error \\
\hline
\end{tabular}




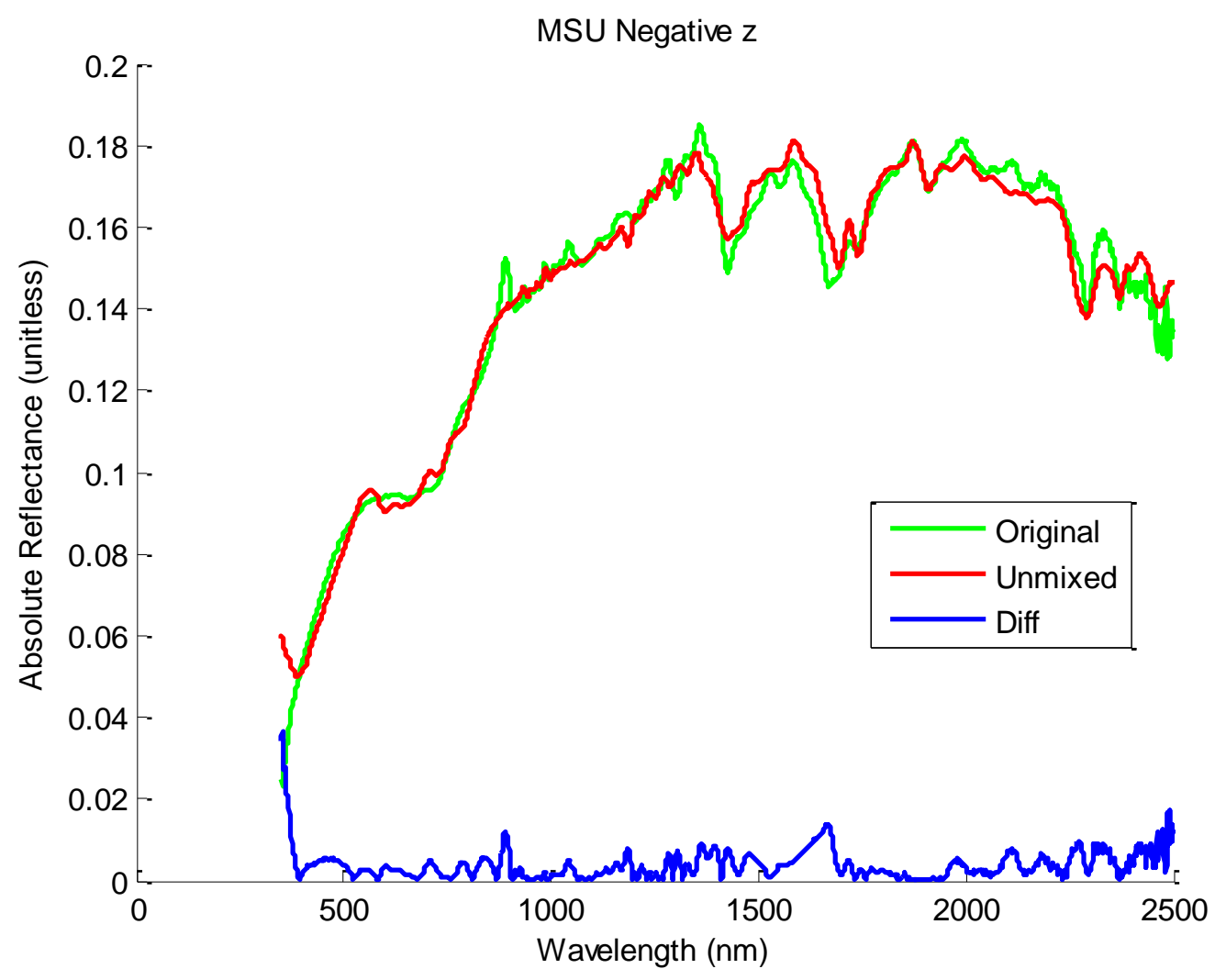

MSU Negative $z$

Unmixing results:

\begin{tabular}{|c|c|}
\hline 0.179855 & Solar Cell 1 \\
\hline 0.059485 & Solar Cell $2 \quad$ (AubieSat) \\
\hline 0.041288 & Green Circuit Board (AubieSat) \\
\hline 0.168319 & Uncoated Green Board (AubieSat) \\
\hline 0.010483 & Solar Cell $3 \quad$ (MSU Sat) \\
\hline 0.115105 & Red Wire with Kapton (MSU Sat) \\
\hline 0.170008 & Red Wire $\quad$ (MSU Sat) \\
\hline 0.017302 & Green Circuit Board (MSU Sat) \\
\hline 0.115126 & White Circuit Board \\
\hline 0.019217 & Black Anodized Aluminum (MSU Sat) \\
\hline 0.061073 & Grey Anodized Aluminum (MSU Sat) \\
\hline 0.000000 & Antenna $\quad$ (MSU Sat) \\
\hline 0.019373 & Solar Cell, no sinusoid feature \\
\hline 0.000000 & Solar Cell, sinusoid feature min TF \\
\hline 0.004688 & Solar Cell, sinusoid feature \\
\hline 0.018679 & Solar Cell, sinusoid feature, alt \\
\hline 0.035395 & Vector Norm Approximation Error \\
\hline
\end{tabular}




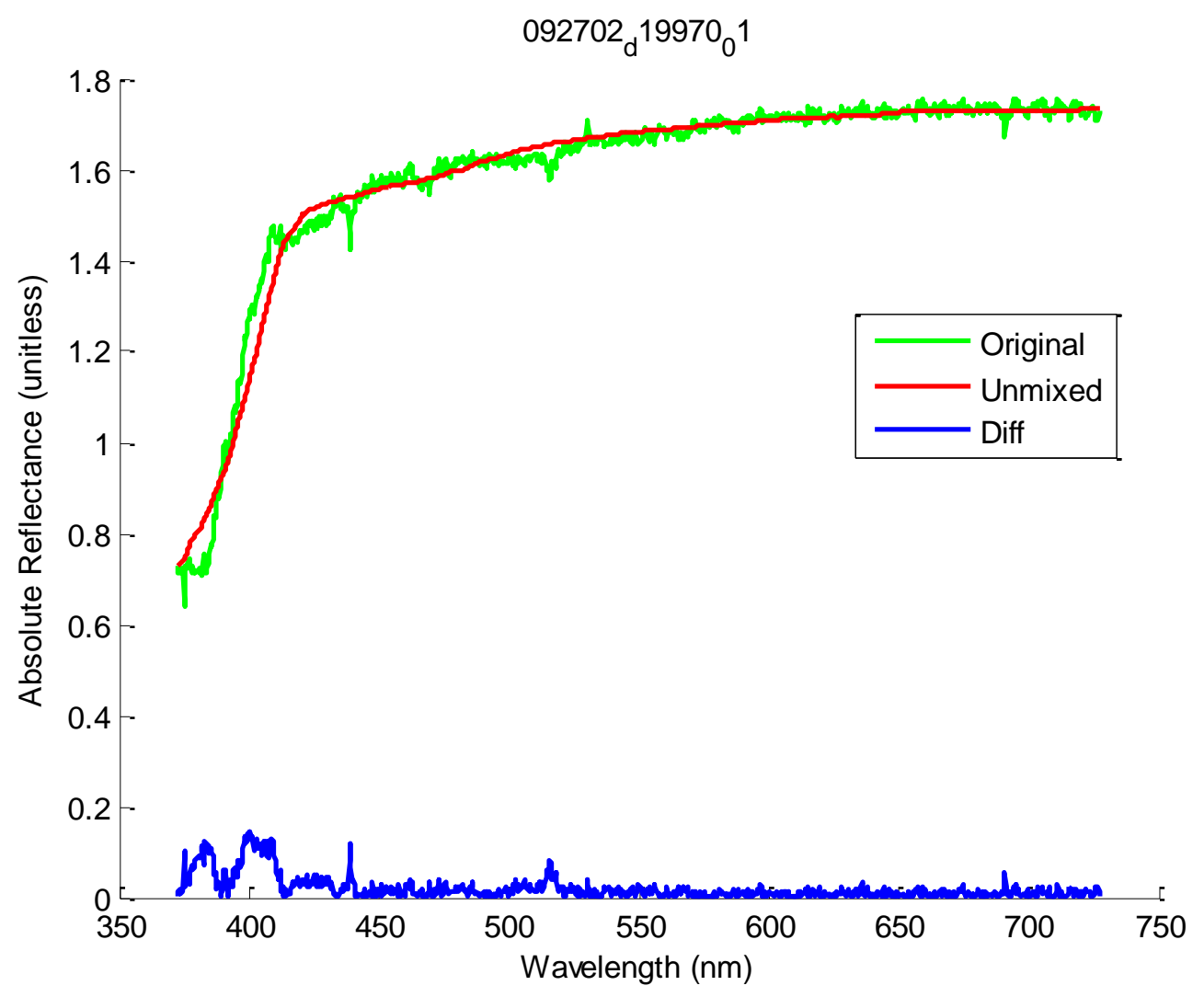

092702_d19970_01

$0.000000 \quad$ Alumholder

$0.669438 \quad$ Carbon Epoxy Nozzle

0.000000 IUS23corrugupsect

$0.221646 \quad$ IUS23midsectll

0.000000 IUS23upsectbetterlt

0.028012 bluecable

0.024297 mligold

0.056609 mligoldback

0.000000 temppresval

0.020741 Percent error (based on vector norm) 


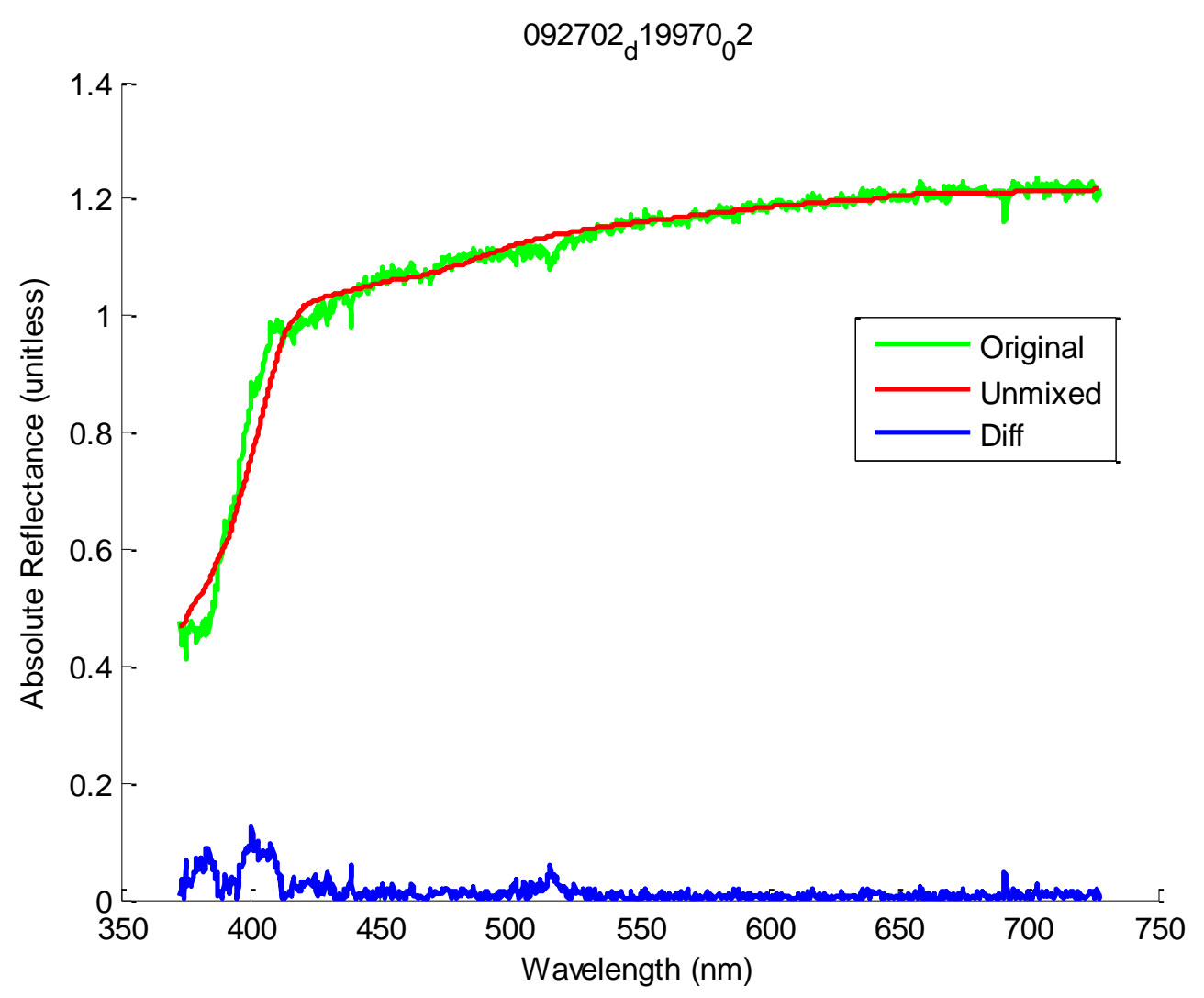

092702_d19970_02

$0.000000 \quad$ Alumholder

$0.625046 \quad$ Carbon Epoxy Nozzle

0.000000 IUS23corrugupsect

0.239049 IUS23midsectll

$0.000000 \quad$ IUS23upsectbetterlt

0.031169 bluecable

0.014662 mligold

0.090074 mligoldback

0.000000 temppresval

0.021203 Percent error (based on vector norm) 


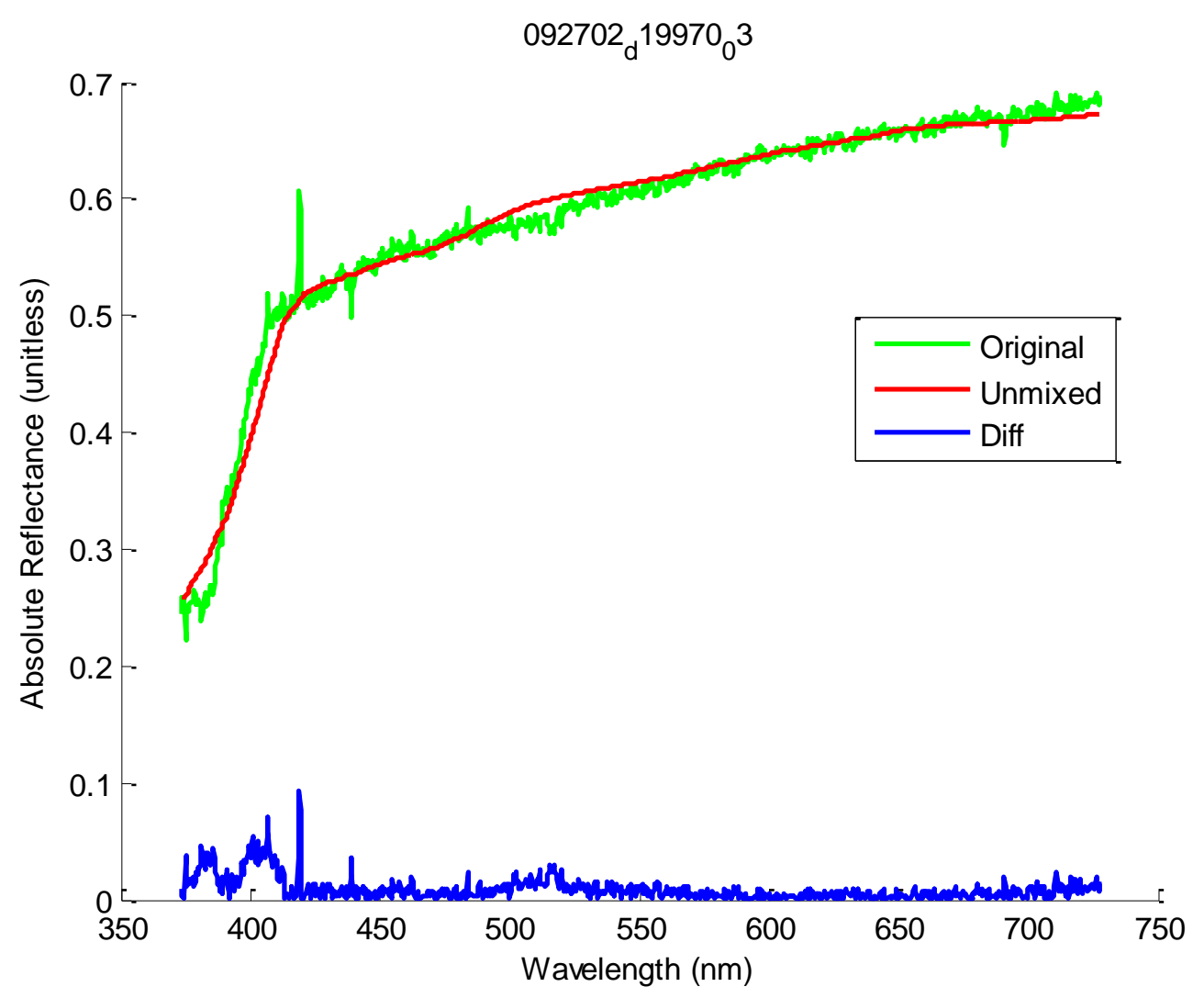

092702_d19970_03

$0.000000 \quad$ Alumholder

$0.607138 \quad$ Carbon Epoxy Nozzle

0.000000 IUS23corrugupsect

$0.192814 \quad$ IUS23midsectll

$0.000000 \quad$ IUS23upsectbetterlt

0.048611 bluecable

0.000000 mligold

0.151437 mligoldback

0.000000 temppresval

0.022748 Percent error (based on vector norm) 


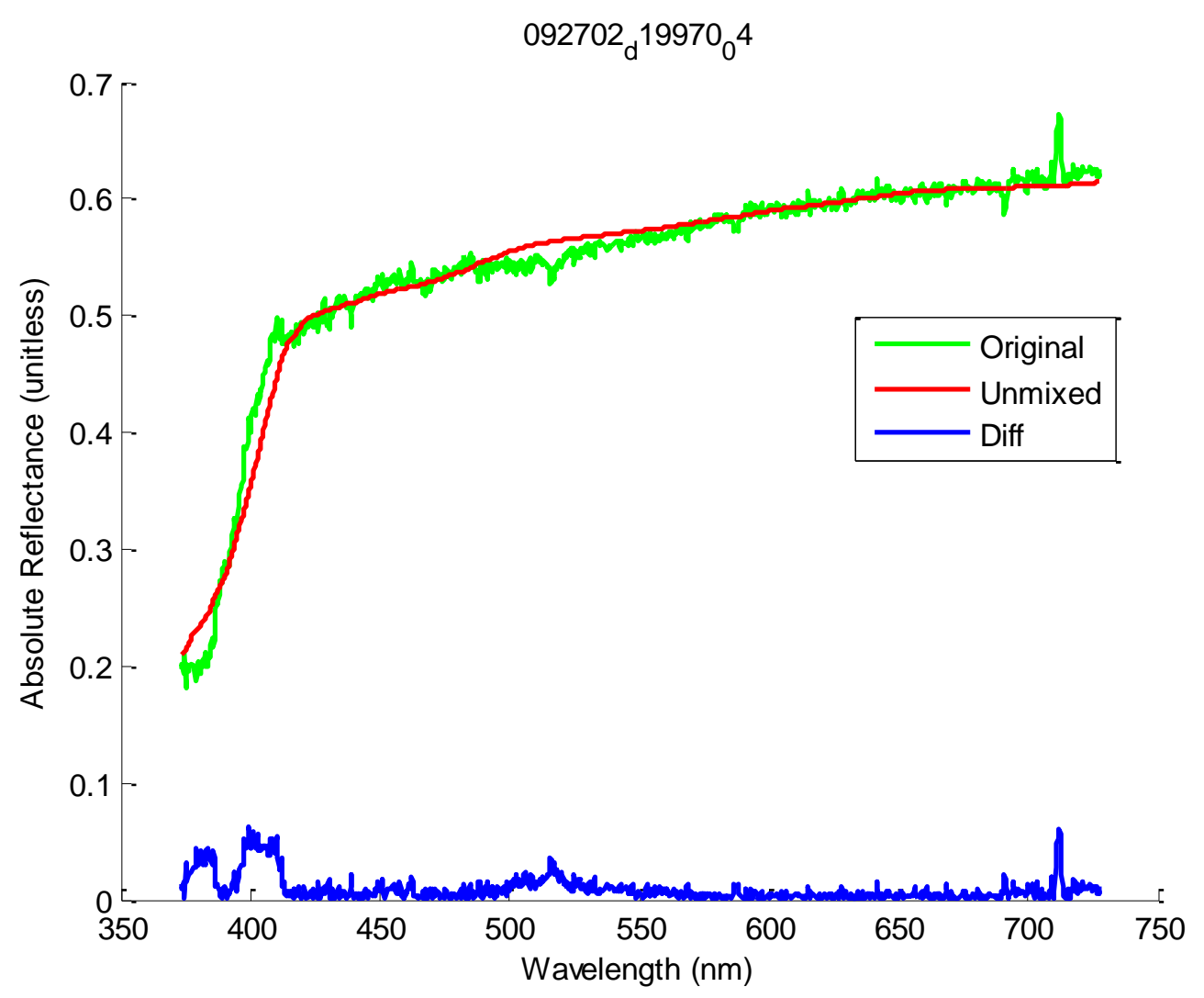

092702_d19970_04

$0.000000 \quad$ Alumholder

$0.551026 \quad$ Carbon Epoxy Nozzle

0.000000 IUS23corrugupsect

$0.263156 \quad$ IUS23midsectll

$0.000000 \quad$ IUS23upsectbetterlt

0.048481 bluecable

0.000000 mligold

0.137337 mligoldback

0.000000 temppresval

0.026317 Percent error (based on vector norm) 


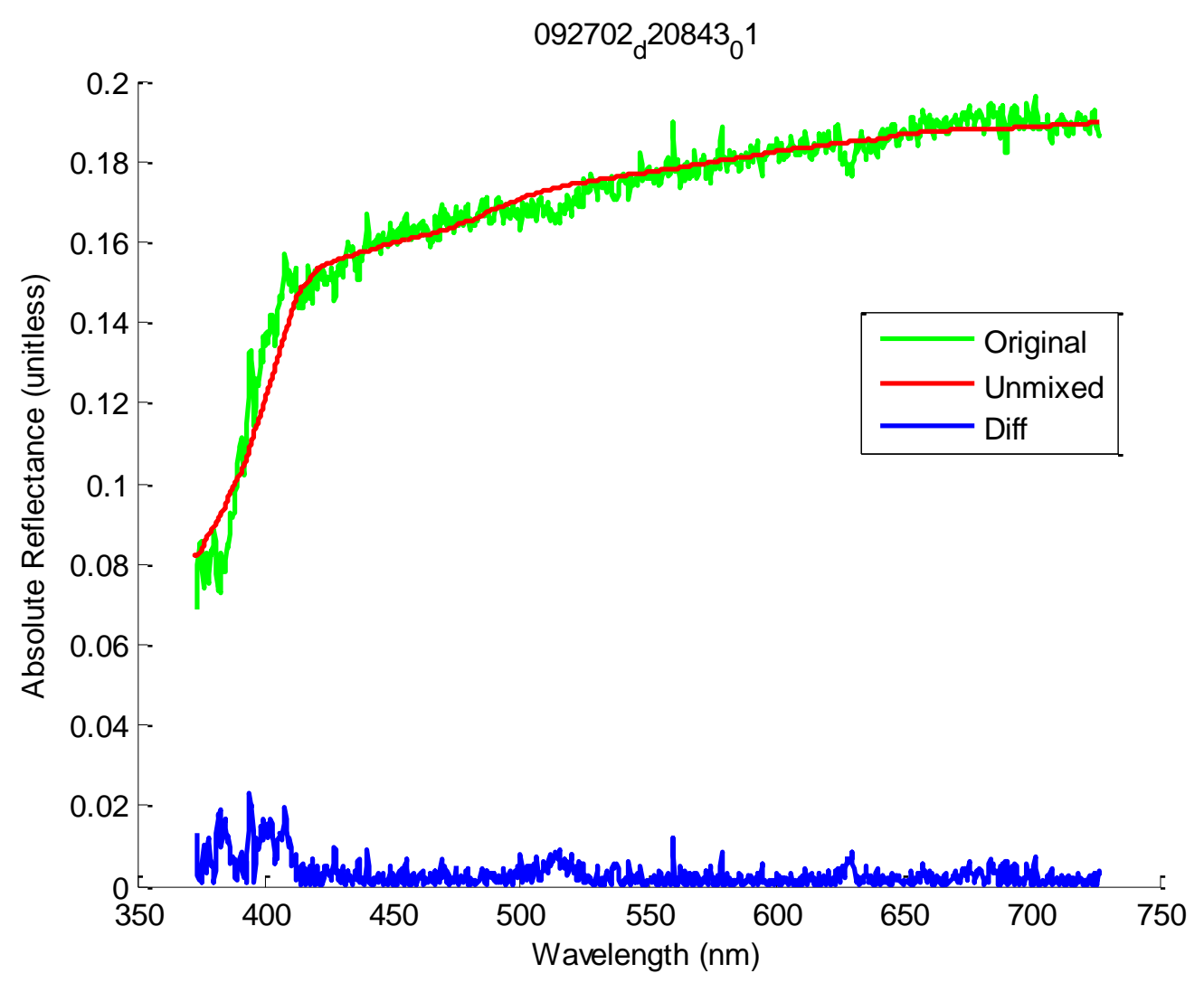

092702_d20843_01

$0.000000 \quad$ Alumholder

$0.686424 \quad$ Carbon Epoxy Nozzle

0.000000 IUS23corrugupsect

0.168548 IUS23midsectll

$0.000000 \quad$ IUS23upsectbetterlt

0.033157 bluecable

0.000000 mligold

0.111871 mligoldback

0.000000 temppresval

0.027349 Percent error (based on vector norm) 


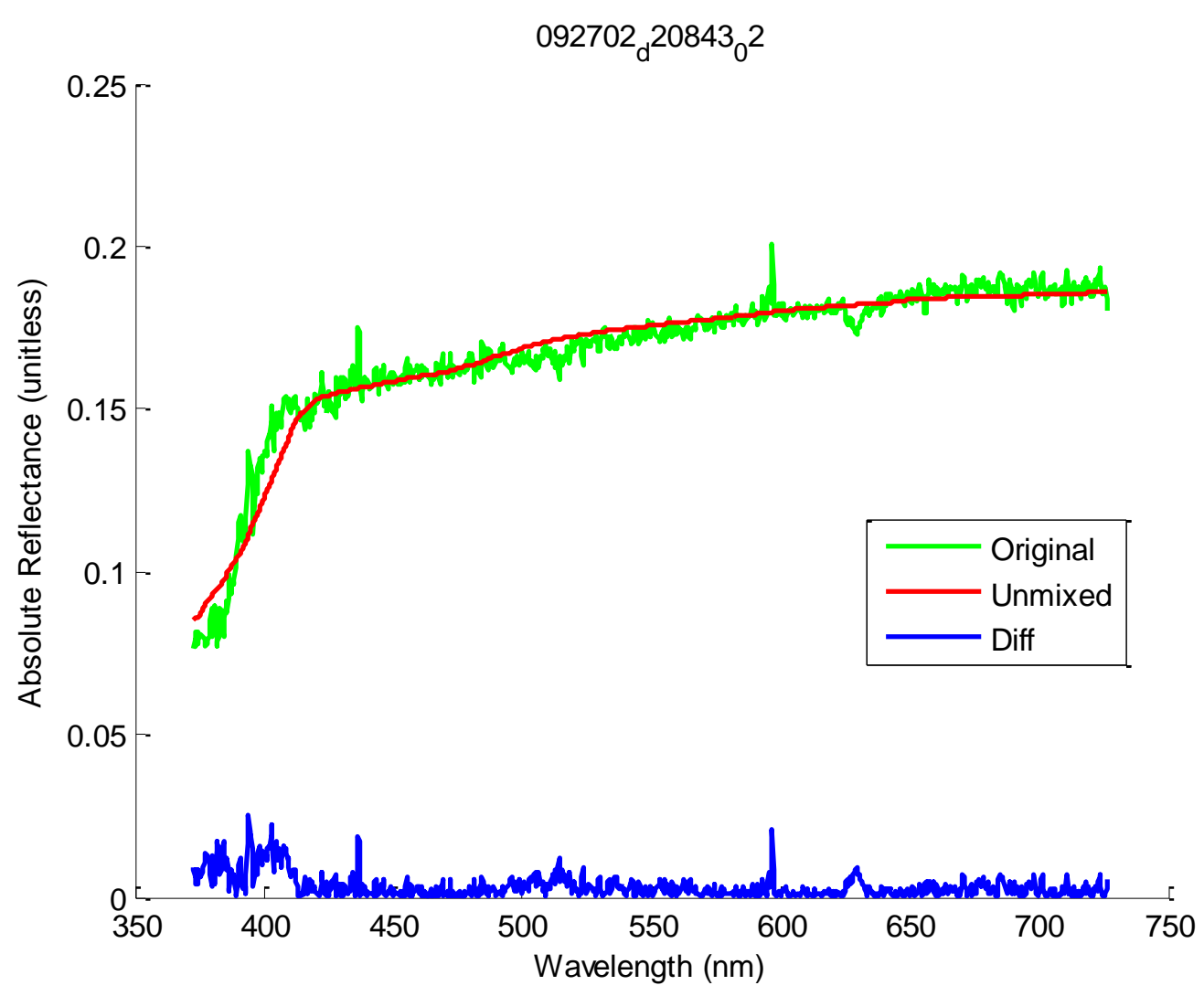

092702_d20843_02

$0.000000 \quad$ Alumholder

$0.726736 \quad$ Carbon Epoxy Nozzle

0.000000 IUS23corrugupsect

$0.156128 \quad$ IUS23midsectll

0.000000 IUS23upsectbetterlt

0.022677 bluecable

$0.000000 \quad$ mligold

0.094459 mligoldback

0.000000 temppresval

0.029356 Percent error (based on vector norm) 


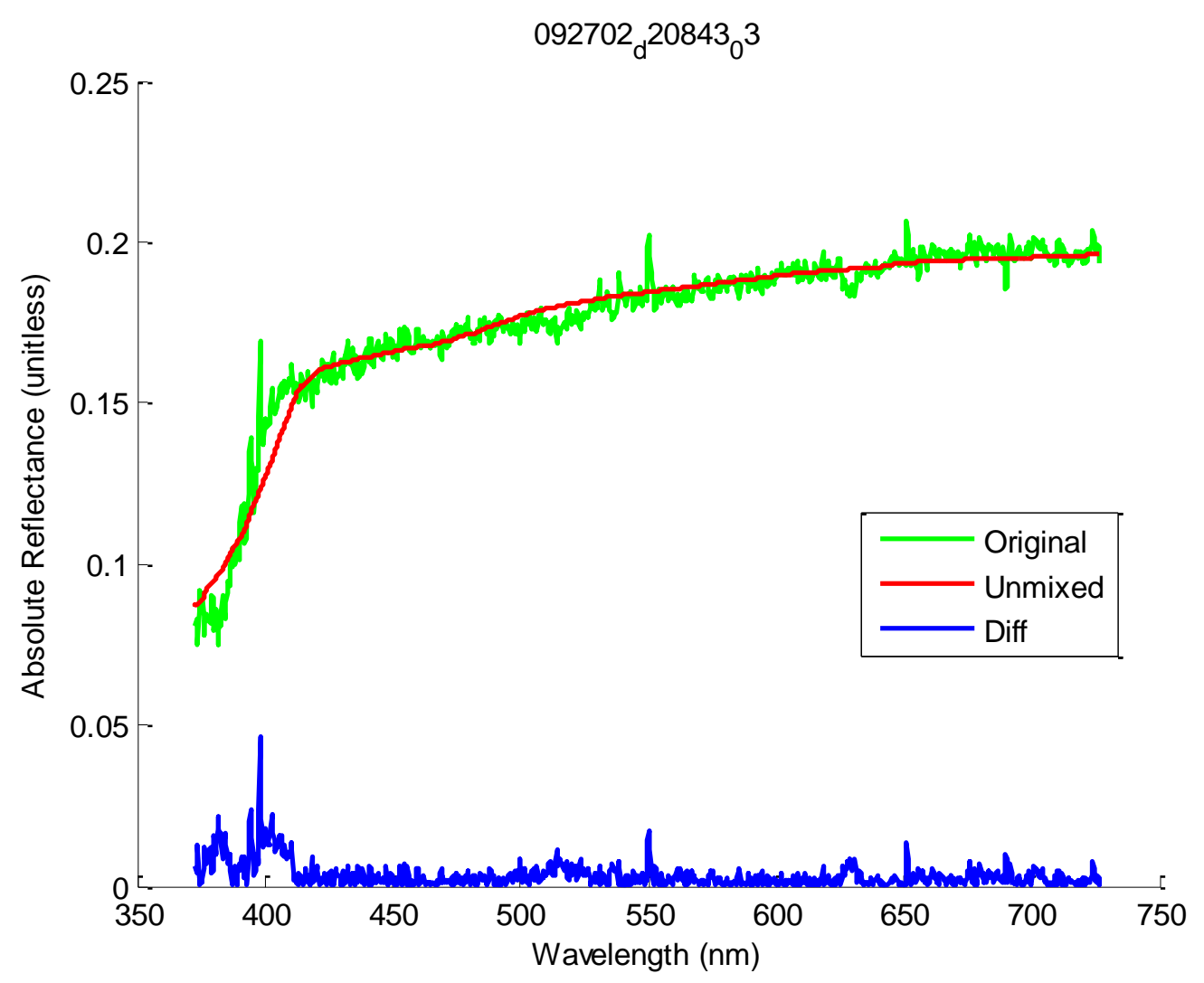

092702_d20843_03

$0.000000 \quad$ Alumholder

$0.708382 \quad$ Carbon Epoxy Nozzle

$0.000000 \quad$ IUS23corrugupsect

$0.164674 \quad$ IUS23midsectll

$0.000000 \quad$ IUS23upsectbetterlt

0.025128 bluecable

0.000000 mligold

0.101816 mligoldback

0.000000 temppresval

0.029283 Percent error (based on vector norm) 
Appendix C |C1 


\section{Appendix C - Notes for future work}

\section{C.1 - Spectroscopy}

First, if a comparison is being made between two or more spectra, it is advisable to minimize the orientation difference between the two measurements. It is recommended to take the two measurements side by side (take one, swap out for the second sample, maintaining the exact same setup). If it is necessary to do before and after measurements on one sample, use some sort of device to mark the position of the sample, probe, and light. This should maintain the orientation to within a few degrees. If the sample is a thermal control material, this may be more difficult, due to curling and warping of the material.

Best procedures for vacuum chamber testing (as of writing, assuming only one sample)

- At all times, handle samples with gloves to avoid contamination.

- Measure initial (no space environment effects) spectrum with spectrometer in the darkroom (For full spectrometer procedure, see Dr. Kira Abercromby).

- Place the samples in the vacuum chamber to be used, set up appropriate apparatus for the test to be performed

- Drop the vacuum chamber to the appropriate pressure for the experiment to be performed 
- Perform the experiment (for outgassing, let sample stay under vacuum for 2 or more days)

- Vent the chamber and remove the samples. Best practice is to measure the samples within 5 minutes of removal from the vacuum chamber. To achieve this, it may be necessary to measure samples one at a time, returning them to vacuum in between measurements.

- Side note on this step, there may be a larger window on these measurements after removal from the chamber, this warrants further study. Perhaps a test with a single material which exhibits an outgassing response, as recommended in Chapter 2, where the material is outgassed, and measured incrementally. This was attempted, but the materials exhibited no change over the $\sim 20$ minute course of the measurement.

- Return the samples to vacuum for storage (or not, depending on the sample type)

A major improvement that could be made for taking measurements on the film materials is a means of holding those materials flat on the measurement surface. This was done by hand for most of the measurements presented in this report. 


\section{C.2 - Unmixing}

Before going into the lessons learned, an explanation for the use of the matlab scripts shown in Appendix C.

UnmixerMain - the basis script which runs the actual unmixer. This is where you run a data parser, and set up the variables and bases for unmixing.

unmixershellcell - the most versatile unmixer, this runs the math and presents the results for review. It expects two inputs, with three fields:

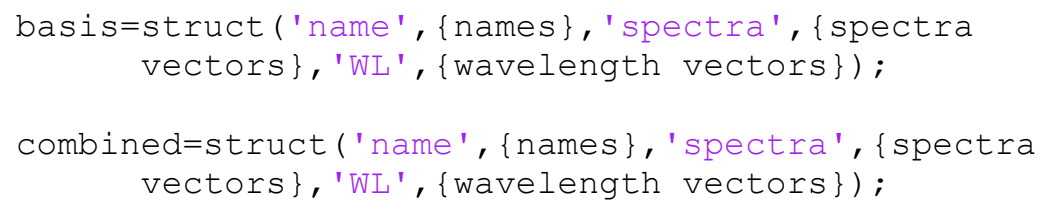

The fields, as shown above, are input as cells, and are name, spectra, and wavelength (WL). This allows it to automatically scale the basis to match the input spectrum for unmixing, and does not require the basis spectra to be on the exact same wavelength region as the input spectra.

Requires the presence of functions unmixer and trimmer to function correctly.

unmixershell - Just like unmixershellcell, but only takes matrices as input (thus requires inputs to be homogenously dimensioned)

Requires the presence of the function unmixer to function correctly. 
spectraread - Reads in spectral data and spits it out as two matrices, one for wavelength data, one for the corresponding values. Useful for initial naming of variables, as it can automatically plot and give a legend for IDing the individual spectra. MatLab's plot broswer is useful for this.

spectrareadcell - Identical to spectraread, except outputs as cells, and outputs name information as well, name comes from either materialslist.txt (must be in data folder) or from the names of the individual data files.

trimmer - This function takes two input spectra, and computes the overlap region, and trims one or both spectra appropriately so they fully overlap.

normalizer - This function takes two input spectra, and selects the best point for normalizing based on the slope around that point, and scales the second spectrum to the first spectrum.

There are a few lessons learned on unmixing. First, one major difficulty, as mentioned earlier is when there are not distinctive features in the spectrum. This leads to a mathematically correct solution, but little means of visual verification of the solution, as the curve fits well, but may not be $100 \%$ certainly the right answer. There are also a few suggestions for mathematical improvements to the solution, first, as mentioned in chapter 3 , the unmixer is solving for the combined value $\mathrm{p}_{\mathrm{i}} \mathrm{B}_{\mathrm{i}}$ rather than the true material proportion. A BRDF model will be necessary to acquire true material proportion $\left(\mathrm{p}_{\mathrm{i}}\right)$. The reason for applying a constrained least 
squares method was to prevent the $\mathrm{p}_{\mathrm{i}} \mathrm{B}_{\mathrm{i}}$ value from returning a negative solution. However, this still includes solutions where both $\mathrm{p}_{\mathrm{i}}$ and $\mathrm{B}_{\mathrm{i}}$ are negative, and it will become necessary to include a means of separating those solutions from future work when $B_{i}$ is solvable.

Another method of note, worth looking into is the use of singular value decompositions in calculating the error factors for each individual material. The singular value decomposition should be able to be used in this fashion to create a possible filter, to remove high-error components, perhaps allowing for more input materials, and less manual filtering. 